Prepared in cooperation with the National Park Service

\title{
Hydrologic Data Summary for the Northeast Creek/Fresh Meadow Estuary, Acadia National Park, Maine, 2000-2001
}

Open-File Report 2007-1035 
Cover. High tide in Northeast Creek, Acadia National Park, Mt. Desert Island, Maine, September 3, 2001 (Photograph by Charles Culbertson, U.S. Geological Survey). 


\section{Hydrologic Data Summary for the Northeast Creek/Fresh Meadow Estuary, Acadia National Park, Maine, 2000-2001}

By James M. Caldwell and Charles W. Culbertson

Prepared in cooperation with the

National Park Service

Open-File Report 2007-1035 


\title{
U.S. Department of the Interior DIRK KEMPTHORNE, Secretary
}

\author{
U.S. Geological Survey \\ Mark D. Myers, Director
}

\section{U.S. Geological Survey, Reston, Virginia: 2007}

For sale by U.S. Geological Survey, Information Services

Box 25286, Denver Federal Center

Denver, CO 80225

For more information about the USGS and its products:

Telephone: 1-888-ASK-USGS

World Wide Web: http://www.usgs.gov/

Any use of trade, product, or firm names in this publication is for descriptive purposes only and does not imply endorsement by the U.S. Government.

Although this report is in the public domain, permission must be secured from the individual copyright owners to reproduce any copyrighted materials contained within this report. 


\section{Contents}

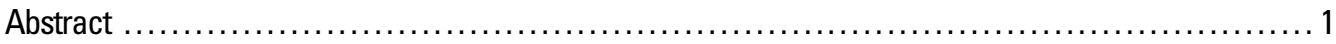

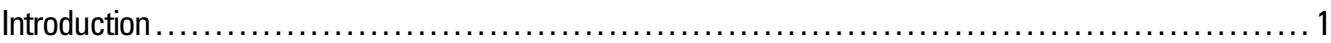

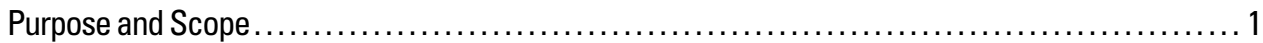

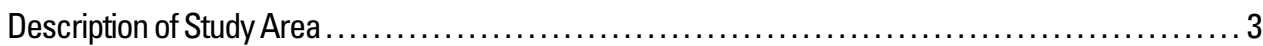

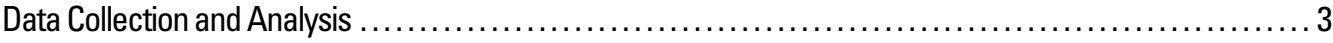

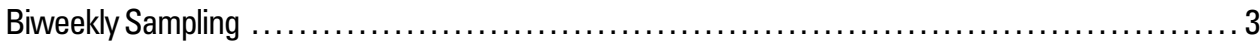

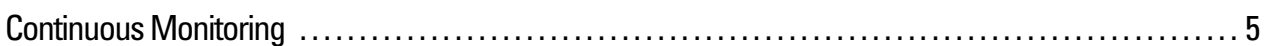

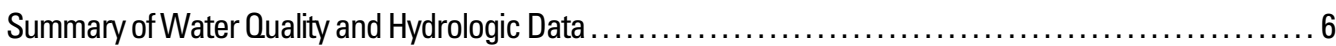

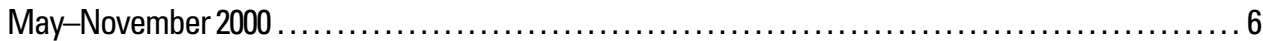

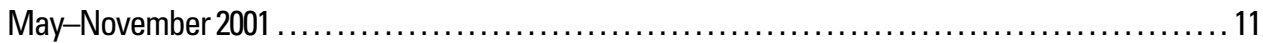

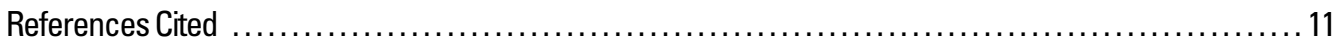

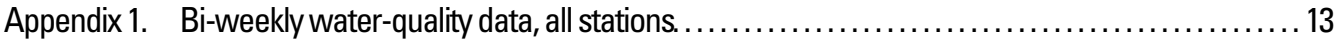

Appendix 2. Water temperature and specific conductance monitored at two depths,

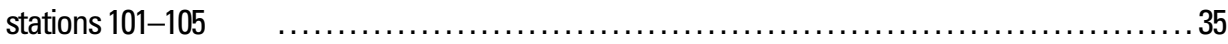

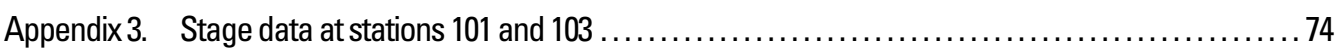

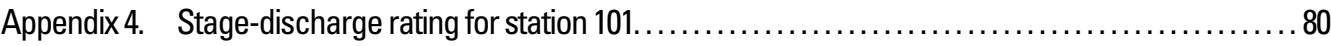

\section{Figures}

1. Map showing location of the Northeast Creek-Fresh Meadow study area, Acadia National Park, Maine......

2-5. Graphs showing:

2. Continuous specific conductance and stage at selected sites during June-0ctober 2000 in the Northeast Creek-Fresh Meadow estuary, Acadia National Park, Maine ..............7

3. Continuous specific conductance and stage at selected sites during June-0ctober 2001 in the Northeast Creek-Fresh Meadow estuary, Acadia National Park, Maine . .............8

4. Total nitrogen, phosphorus, and chlorophyll-a concentrations during May-November 2000 at stations 100-105 in the Northeast Creek-Fresh Meadow estuary, Acadia National Park, Maine.

5. Total nitrogen, phosphorus, and chlorophyll-a concentrations during May-November 2001 at stations 100-105 in the Northeast Creek-Fresh Meadow estuary, Acadia National Park, Maine.

\section{Tables}

1. Sites sampled along Northeast Creek near Bar Harbor, Maine .............................

2. Constituents and minimum reporting limits for water-quality sample analyses conducted as

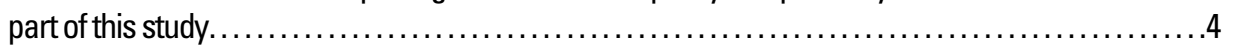

3. Analytical methods used for selected water-quality constituents in this study . ...............5 


\section{Conversion Factors, Datum, and Other Abbreviations}

\begin{tabular}{|c|c|c|}
\hline Multiply & By & To obtain \\
\hline \multicolumn{3}{|c|}{ Length } \\
\hline centimeter $(\mathrm{cm})$ & 0.3937 & inch (in.) \\
\hline meter $(m)$ & 3.281 & foot (ft.) \\
\hline kilometer (km) & .6214 & mile (mi.) \\
\hline millimeter (mm) & .03937 & inch (in.) \\
\hline \multicolumn{3}{|c|}{ Area } \\
\hline square kilometer $\left(\mathrm{km}^{2}\right)$ & .3861 & square mile $\left(\mathrm{mi}^{2}\right)$ \\
\hline \multicolumn{3}{|c|}{ Volume } \\
\hline milliliter $(\mathrm{mL})$ & .0338 & fluid ounce \\
\hline liter $(\mathrm{L})$ & .2642 & gallon \\
\hline cubic meter $\left(\mathrm{m}^{3}\right)$ & $8.110 \times 10^{-4}$ & acre-foot \\
\hline cubic meter $\left(\mathrm{m}^{3}\right)$ & 35.31 & cubic foot \\
\hline \multicolumn{3}{|c|}{ Mass } \\
\hline milligram (mg) & $3.527 \times 10^{-8}$ & ounce \\
\hline kilogram (kg) & 2.205 & pound \\
\hline $\begin{array}{l}\text { kilogram per square kilometer per year } \\
\qquad\left(\mathrm{kg} / \mathrm{km}^{2} / \mathrm{yr}\right)\end{array}$ & 5.710 & pound per square mile per year \\
\hline \multicolumn{3}{|c|}{ Flow rate } \\
\hline cubic foot per second $\left(\mathrm{ft}^{3} / \mathrm{s}\right)$ & .02832 & cubic meter per second \\
\hline
\end{tabular}

Temperature in degrees Celsius $\left({ }^{\circ} \mathrm{C}\right)$ may be converted to temperature in degrees Fahrenheit ( ${ }^{\circ} \mathrm{F}$ ) as follows:

$$
{ }^{\circ} \mathrm{F}=\left(1.8 \times{ }^{\circ} \mathrm{C}\right)+32
$$

Altitude, as used in this report, refers to distance above or below sea level.

Specific conductance is given in microsiemens per centimeter at 25 degrees Celsius $\left(\mu \mathrm{S} / \mathrm{cm}\right.$ at $25^{\circ} \mathrm{C}$ ). Concentrations of chemical constituents in water are given either in milligrams per liter $(\mathrm{mg} / \mathrm{L})$ or in micrograms per liter $(\mu \mathrm{g} / \mathrm{L})$.

OTHER ABBREVIATIONS USED IN THIS REPORT:

$\mathrm{mg} / \mathrm{L} \quad$ milligrams per liter

$\mu \mathrm{g} / \mathrm{L} \quad$ micrograms per liter

WRI Water Research Institute

USGS United States Geological Survey

UM University of Maine

DOC dissolved organic carbon

DIC dissolved inorganic carbon

ICP-MS inductively coupled plasma mass spectrometry

DIN dissolved inorganic nitrogen

DON dissolved organic nitrogen 


\title{
Hydrologic Data Summary for the Northeast Creek/ Fresh Meadow Estuary, Acadia National Park, Maine, 2000-2001
}

\author{
By James M. Caldwell and Charles W. Culbertson
}

\section{Abstract}

The U.S. Geological Survey, in cooperation with the National Park Service, collected data in Northeast Creek estuary, Mt. Desert Island, Maine, to establish baseline water-quality conditions including estuarine nutrient concentrations. Five sampling sites in Northeast Creek were established and monitored continuously for temperature and specific conductance during May to November, 2000 and 2001. Stream stage, which was affected by ocean tidal dynamics, was recorded at the most downstream site and at one upstream site. Discrete water samples for nutrient concentrations were collected biweekly during May to November, 2000 and 2001, at the five sampling sites, and an additional site seaward of the estuary mouth. Results indicated that the salinity regime of Northeast Creek estuary is dynamic and highly regulated by strong seasonal variations in freshwater runoff, as well as limited seawater exchange caused by a constriction at the bridge, at the downstream end of the estuary. Oligohaline conditions ( $0.5-5$ practical salinity units) occasionally extend to the estuary mouth. During other periods oligohaline and mesohaline (5-20 practical salinity units) conditions exist in some areas of the estuary; polyhaline/marine (20-35 practical salinity units) conditions occasionally exist near the mouth. A saltwater wedge in the bottom water, due to density stratification, was observed to migrate upstream as fresh surface-water inputs diminished during the onset of summer low-flow conditions. Although specific conductance ranged widely at most sites because of tidal influences, other waterquality constituents, including nutrient and chlorophyll- $a$ concentrations, exhibited seasonal distribution patterns in which maximum levels generally occurred in early to mid-summer and again in the fall over both field seasons.

\section{Introduction}

Acadia National Park is located on Mt. Desert Island, on the central coast of Maine (fig. 1). The area has experienced rapid residential development outside of park boundaries since the late 1990's. Changes in water quality on Mt. Desert Island primarily have been attributed to air pollution and residential development within the watersheds (Neckles and others, 2003; Nielsen, 2002; Doering and others, 1995). Acadia's Water Resources Management Plan (Acadia National Park, 2000; Manski, 1998) identifies accelerated rates of freshwater and coastal marine eutrophication as a priority water-quality issue and one of the Park's most important resource management challenges. A substantial amount of recent residential development on Mt. Desert Island has occurred within the Northeast Creek/Fresh Meadow watershed (Nielsen, 2002), prompting concerns by resource managers at the Park that Northeast Creek is susceptible to accelerated nutrient enrichment and eutrophication. To address these concerns, in 2000 the USGS (U.S. Geological Survey) initiated a study, in cooperation with the National Park Service, to establish baseline water-quality conditions for Northeast Creek/Fresh Meadow watershed, Mt. Desert Island, Maine. The study will allow the effects of additional land use and land cover changes in the watershed to be assessed, and is part of an ongoing effort by the USGS to characterize water resources in Acadia National Park. Additional hydrologic data collected in Acadia National Park during this time period, including streamflow and water-quality data from four perennial streams that flow to Northeast Creek are found in Nielsen and others (2000), Stewart and others (2001), and Nielsen (2002).

\section{Purpose and Scope}

This report presents and describes all data collected during the baseline study of Northeast Creek/Fresh Meadow estuary and summarizes all methods and techniques used for data collection and analysis. Data were collected at six sites within the Northeast Creek/Fresh meadow estuary during ice-free months (May-November) of 2000-01. Continuous water-temperature and specific conductance data were collected at stations 101, $102,103,104$, and 105 at two discrete depths. Continuous stage data were collected at stations 101 and 103 (fig. 1). Data collection also included biweekly water-quality sampling at stations $100-105$. Samples were analyzed for 18 water-quality constitu- 


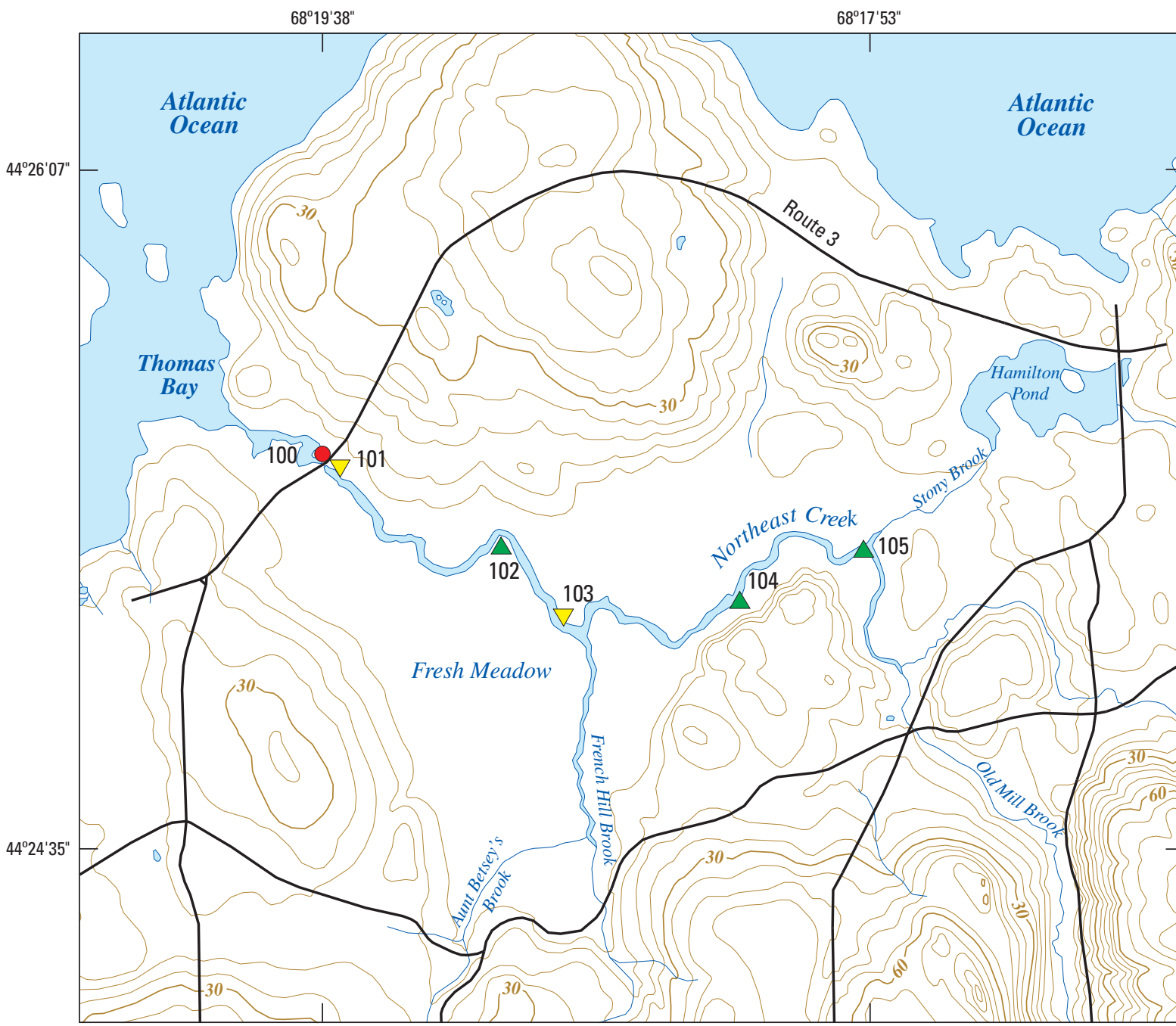

Base from U.S. Geological Survey

Digital line graph Salsbury Cove, 1981, 1:24,000

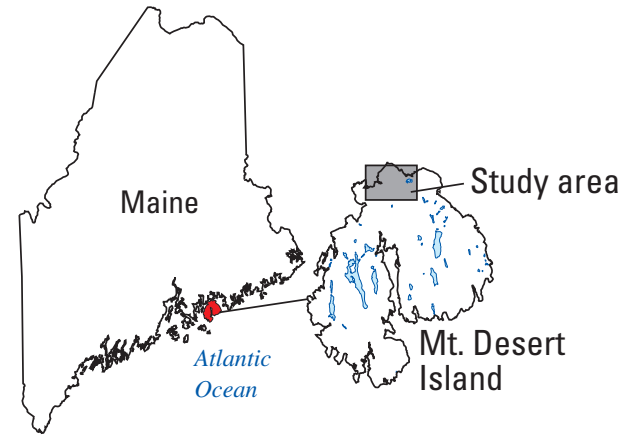

0

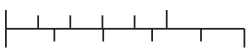

EXPLANATION

$-30-$ Topographic contour-Contour interval 6 meters

Monitoring sites

100 Biweekly water quality

$\triangle^{102}$ Biweekly water quality and continuous temperature and specific conductance

$\nabla^{101}$ Biweekly water quality and continuous temperature, specific conductance, and stage

Figure 1. Location of the Northeast Creek-Fresh Meadow study area, Acadia National Park, Maine. 
ents, including nutrients, silica, and chlorophyll- $a$. Time-series graphs were constructed to compare specific conductance and stage data, and to illustrate the distribution of selected waterquality constituents. All data collected during the study, including quality-assurance records and a stage-discharge rating for one site, are presented as tables in the appendixes.

\section{Description of Study Area}

The study area is located on Mt. Desert Island, Maine (fig. 1). Topography ranges from hills with slopes of about 5 percent to mountains with slopes greater than 60 percent. Annual rainfall is about $50 \mathrm{in} / \mathrm{yr}$ (National Oceanic and Atmospheric Administration, 1979-1999), about 10 in. greater than the average for other coastal areas in Maine. National Park Service land constitutes more than half the area of Mt. Desert Island. As of 2000, the year-round population of the island was 8,770 (U.S. Census Bureau, 2001). The island population increases substantially each summer due to the influx of seasonal residents. Acadia National Park receives about 2.5 million visitors per year.

The Northeast Creek/Fresh Meadow wetland, located on the northern lobe of Mt. Desert Island (fig. 1), is the largest estuarine wetland on the island, and a primary physiographic feature of the park. Although a substantial part of the estuarine wetland is within park boundaries, much of the Northeast Creek watershed lies outside the park. Surface-water inputs to the wetland include four perennial streams and three intermittent streams.

Areas adjacent to the wetland do not contribute any substantial channelized surface-water flow to the wetland or creek; however, they may contribute shallow ground-water flow to the wetland (Nielsen, 2002). The four perennial streams and their drainage areas are Stony Brook $\left(2.60 \mathrm{mi}^{2}\right)$, Old Mill Brook $\left(2.37 \mathrm{mi}^{2}\right)$, Aunt Betsy's Brook $\left(0.63 \mathrm{mi}^{2}\right)$, and French Hill Brook $\left(0.54 \mathrm{mi}^{2}\right)$. The three intermittent streams have a combined drainage area of $1.31 \mathrm{mi}^{2}$. The Northeast Creek/Fresh Meadow wetland has an area of $0.71 \mathrm{mi}^{2}$; the surface area of
Northeast Creek itself is estimated to be $0.05 \mathrm{mi}^{2}$ (Nielsen, 2002).

The outlet of Northeast Creek flows into Thomas Bay (fig. 1), and is constricted vertically by remnants of an old rock dam that lie slightly below the mean high tide line (Nielsen, 2002), and horizontally by bridge abutments. Tidal flow into and out of Northeast Creek varies as a function of the biweekly tidal cycle. During the flooding spring tides, seawater flows over the control structure into Northeast Creek with seaward flow occurring during the subsequent ebbing tide. During neap tides the estuary receives little or no seawater input and the tidal amplitude is dampened or undetectable.

\section{Data Collection and Analysis}

\section{Biweekly Sampling}

Biweekly water-quality sampling for nutrients, chlorophyll- $a$, and water quality parameters of temperature, specific conductance, $\mathrm{pH}$, and dissolved oxygen, was conducted at 6 sites in the study area (fig. 1; table 1) and included Van-Dornstyle sampling and in situ water-quality measurements. All water chemistry analyses except for chlorophyll- $a$ were performed at the Water Research Institute (WRI) inorganic chemistry laboratory at the University of Maine (UM). The laboratory participates in the USGS standard reference sample program (Long and others, 1998), which is used to detect and correct possible analytical deficiencies and problems. The UM laboratory met all USGS analytical requirements for the constituents analyzed for this study. Chlorophyll- $a$ samples were analyzed at the Bigelow Laboratory for Ocean Sciences, East Boothbay Harbor, ME. Quality assurance procedures for chlorophyll- $a$ analyses included collection of random duplicate samples, and performing appropriate equipment, filter, and solvent blank analyses at each biweekly sampling interval.

Table 1. Sites sampled along Northeast Creek near Bar Harbor, Maine.

[dd, degrees; mm, minutes; ss, seconds]

\begin{tabular}{|c|c|c|c|c|c|c|c|}
\hline $\begin{array}{l}\text { USGS station } \\
\text { identifier }\end{array}$ & $\begin{array}{c}\text { Site } \\
\text { number on } \\
\text { figure } 1\end{array}$ & $\begin{array}{c}\text { Latitude } \\
\text { (dd mm ss) }\end{array}$ & $\begin{array}{l}\text { Longitude } \\
\text { (dd mm ss) }\end{array}$ & $\begin{array}{l}\text { Bi-weekly } \\
\text { samples }\end{array}$ & Stage & $\begin{array}{l}\text { 15-minute } \\
\text { recorded } \\
\text { conductance }\end{array}$ & $\begin{array}{c}\text { 15-minute } \\
\text { recorded } \\
\text { temperature }\end{array}$ \\
\hline 442530068193901 & 100 & 442530 & 681939 & $\mathrm{x}$ & & & \\
\hline 01022820 & 101 & 442528 & 681936 & $\mathrm{x}$ & $\mathrm{x}$ & $\mathrm{x}$ & $\mathrm{x}$ \\
\hline 442517068190501 & 102 & 442517 & 681905 & $\mathrm{x}$ & & $\mathrm{x}$ & $\mathrm{x}$ \\
\hline 442507068185301 & 103 & 442507 & 681853 & $\mathrm{x}$ & $\mathrm{x}$ & $\mathrm{x}$ & $\mathrm{x}$ \\
\hline 442509068181901 & 104 & 442509 & 681819 & $\mathrm{x}$ & & $\mathrm{x}$ & $\mathrm{x}$ \\
\hline 442516068175501 & 105 & 442516 & 681755 & $\mathrm{x}$ & & $\mathrm{x}$ & $\mathrm{x}$ \\
\hline
\end{tabular}




\section{Hydrologic Data Summary for the Northeast Creek/Fresh Meadow Estuary, Acadia National Park, Maine, 2000-2001}

Biweekly water-quality samples were collected $0.2 \mathrm{~m}$ below the water surface and about $0.2 \mathrm{~m}$ from the streambed using a 2-L Van-Dorn-style water sampler, and combined in a churn splitter. Water column depth at each of the sites typically averaged about $1 \mathrm{~m}$, although depths varied because of tidal cycle and changing flow conditions from tributaries. Composite samples were subsampled into amber or clear polypropylene or glass containers, depending on type of analysis, and chilled immediately on ice. Samples for dissolved constituents were filtered through a $0.45-\mu \mathrm{m}$ Nuclepore polycarbonate filter within 6 hours of collection, and transported on ice to the WRI inorganic chemistry laboratory for analysis.

In-situ water-quality measurements of temperature, $\mathrm{pH}$, specific conductance, and dissolved oxygen were made about $0.2 \mathrm{~m}$ below the water surface and about $0.2 \mathrm{~m}$ from the streambed at each site at the time of sample collection using a Hydrolab Minisonde 4a multi-parameter water-quality sensor. Calibration of the water-quality sensor was performed twice daily using standards selected to bracket the expected range of field values.

Samples for chlorophyll- $a$ analysis were collected in amber 2-L polypropylene Nalgene bottles from composite water column samples, as described above, and chilled immediately on ice. Triplicate sub-samples were filtered in the labora- tory under low light onto 47-mm Whatman GF/F glass-fiber filters (effective pore size $=0.7 \mu \mathrm{m}$ ) within 6 hours of collection, and stored frozen $\left(-40^{\circ} \mathrm{C}\right)$ until extracted and analyzed within 4 weeks of being filtered.

The results of water-quality analyses performed on these samples and the associated field readings are shown in appendix 1 . The tables are organized by calendar year, station and date. The quality assurance data that accompanies those results are shown at the end of appendix 1; also organized by calendar year, station and date.

Samples were analyzed for total and dissolved concentrations of nutrients (nitrogen and phosphorus), chlorophyll- $a$, and dissolved silica. Total nitrogen analyses included particulate and dissolved organic and inorganic nitrogen; dissolved nitrogen analyses included only dissolved inorganic species (ammonium and nitrate in 2000; ammonium, nitrate and nitrite in 2001). Total phosphorous analyses included particulate and dissolved organic and inorganic phosphorous; dissolved phosphorous analyses included only ortho-phosphate. During the 2000 season samples also were analyzed for selected major ions, and dissolved organic and inorganic carbon. The constituents analyzed and their associated reporting limits are presented in table 2 . Table 3 provides further details about the analytical methods used for selected constituents.

Table 2. Constituents and minimum reporting limits for water-quality sample analyses conducted as part of this study.

$\left[{ }^{\circ} \mathrm{C}\right.$, degrees Celsius; na, not applicable; $\mathrm{ms} / \mathrm{cm}$, microsiemens per centimeter; $\mathrm{mg} / \mathrm{L}$, milligrams per liter; $\mu \mathrm{g} / \mathrm{L}$, micrograms per liter; $\mathrm{N}$, nitrogen; P, Phosphorus; NWIS, National Water Information System]

\begin{tabular}{|c|c|c|}
\hline $\begin{array}{c}\text { USGS NWIS } \\
\text { parameter code }\end{array}$ & $\begin{array}{l}\text { Constituent } \\
\text { (units) }\end{array}$ & $\begin{array}{l}\text { Minimum } \\
\text { reporting limit }\end{array}$ \\
\hline 00010 & Temperature, water $\left({ }^{\circ} \mathrm{C}\right)$ & 0.1 \\
\hline 00095 & Specific conductance, field ( $\mu \mathrm{s} / \mathrm{cm}$ at $25^{\circ} \mathrm{C}$ ) & 1. \\
\hline 00300 & Oxygen, dissolved (mg/L) & 0.1 \\
\hline 00301 & Oxygen, dissolved (percent of saturation) & na \\
\hline 00400 & $\mathrm{pH}$, field, unfiltered water (standard units) & na \\
\hline 00915 & Calcium, dissolved (mg/L) & 0.05 \\
\hline 00925 & Magnesium, dissolved (mg/L) & 0.05 \\
\hline 00930 & Sodium, dissolved (mg/L) & 0.05 \\
\hline 00935 & Potassium, dissolved (mg/L) & 0.05 \\
\hline 00940 & Chloride, dissolved (mg/L) & 0.1 \\
\hline 00945 & Sulfate, dissolved $\left(\mathrm{mg} / \mathrm{L}\right.$ as $\left.\mathrm{SO}_{4}\right)$ & 0.2 \\
\hline 00955 & Silica, dissolved (mg/L as $\left.\mathrm{SiO}_{2}\right)$ & 0.5 \\
\hline 00618 & Nitrogen, nitrate, dissolved (mg/L as $\mathrm{N})$ & 0.01 \\
\hline 00631 & Nitrogen, nitrite plus nitrate, dissolved ( $\mathrm{mg} / \mathrm{L}$ as $\mathrm{N})$ & 0.5 \\
\hline 71846 & Nitrogen, ammonia, dissolved ( $\mathrm{mg} / \mathrm{L}$ as $\left.\mathrm{NH}_{4}\right)$ & 0.05 \\
\hline 00600 & Nitrogen, total (mg/L as N) & 0.01 \\
\hline 00671 & Phosphorus, orthophosphate, dissolved (mg/L as $\mathrm{P}$ ) & 0.1 \\
\hline 00665 & Phosphorus, total (mg/L) & 0.001 \\
\hline 00681 & Carbon, organic, dissolved (mg/L) & 0.5 \\
\hline 00691 & Carbon, inorganic, dissolved (mg/L) & 0.5 \\
\hline 32209 & Chlorophyll- $a(\mu \mathrm{g} / \mathrm{L})$ & 0.1 \\
\hline
\end{tabular}


Table 3. Analytical methods used for selected water-quality constituents in this study.

[EPA, Environmental Protection Agency; nm, nanometer]

\begin{tabular}{|c|c|c|c|c|}
\hline Constituent & $\begin{array}{c}\text { Storage } \\
\text { temper- } \\
\text { ature } \\
\text { (degrees } \\
\text { Celsius) }\end{array}$ & $\begin{array}{l}\text { Maximum } \\
\text { storage } \\
\text { time } \\
\text { prior to } \\
\text { analysis } \\
\text { (days) }\end{array}$ & Analytical method & Reference \\
\hline Ammonium $^{1}$ & 4 & 28 & Colorimetry at $660 \mathrm{~nm}$ using an autoanalyzer & Morrison (1989) \\
\hline Dissolved anions $^{1}$ & 4 & 28 & Ion Chromatography EPA Method 300.0 & $\begin{array}{l}\text { U.S. Environmental Protection Agency } \\
(1984,1993)\end{array}$ \\
\hline Dissolved cations ${ }^{1}$ & 4 & 28 & $\begin{array}{l}\text { Ultrasonic nebulization and inductively } \\
\text { coupled plasma with mass spectroscopy } \\
\text { detection }\end{array}$ & $\begin{array}{l}\text { U.S. Environmental Protection Agency } \\
\text { (1979) }\end{array}$ \\
\hline Dissolved silica $^{1}$ & 4 & 28 & $\begin{array}{l}\text { Colorimetry, using standard method } \\
4500-\mathrm{SiO}_{2}\end{array}$ & $\begin{array}{l}\text { American Public Health Association } \\
\text { and others (1998) }\end{array}$ \\
\hline Nitrate plus Nitrite ${ }^{1,3}$ & 4 & 7 & $\begin{array}{l}\text { Colorimetry, EPA method } 353.2 \text {, Cadmium } \\
\text { reduction }\end{array}$ & $\begin{array}{l}\text { U.S. Environmental Protection Agency } \\
\text { (1979) }\end{array}$ \\
\hline Total nitrogen & 4 & 28 & $\begin{array}{l}\text { Alkaline persulfate digestion followed by } \\
\text { colorimetric detection at } 540 \mathrm{~nm} \text { using an } \\
\text { autoanalyzer }\end{array}$ & $\begin{array}{l}\text { U.S. Environmental Protection Agency } \\
\text { (1987) }\end{array}$ \\
\hline Total phosphorus & 4 & 28 & Standard method 4500-E & $\begin{array}{l}\text { American Public Health Association } \\
\text { and others (1998) }\end{array}$ \\
\hline
\end{tabular}

\section{Continuous Monitoring}

Continuous temperature, specific conductance, and stage data were collected at a time interval of 15-minutes. Water temperature and specific conductance were monitored continuously at 2 depths at stations 101-105 during ice-free months in the Northeast Creek estuary in 2000 and 2001. The temperature/ conductivity sensors at these sites were installed at fixed depths, about $0.2 \mathrm{~m}$ below the mean low tide water surface, and about $0.2 \mathrm{~m}$ above the streambed. Stream stage was recorded to the nearest 0.01 foot at station 101 from May 2000 to November 2001 using an in-line pressure sensor/nitrogen conoflow system mounted on the stream bottom (Carter and Davidian, 1968). For comparison with station 101, relative stage was measured at 15-minute intervals at station 103 from September to November 2000 and May to November 2001 using a submersible pressure transducer.
A Wetlabs ECO-DFLS submersible recording fluorometer (Wetlabs Inc., Philomath, OR) was installed at a depth of about $0.4 \mathrm{~m}$ below the mean low tide water surface at station 102 during much of this project as a means to estimate changes in chlorophyll- $a$ concentration between sampling visits. As this is a fixed-depth instrument, the measurements reflect chlorophyll- $a$ concentration at that depth, and may not represent chlorophyll- $a$ concentrations throughout the water column. These data were collected for experimental purposes and are not included in this report; however they are available upon request at the USGS Water Science Center in Augusta, Maine.

Six stream discharge measurements were made at station 101 over a range of hydrologic conditions and used with corresponding stream stage values to develop a stage-discharge relation (Rantz and others, 1982), which is shown later. Although computation of outflow (positive discharge) at station 101 was beyond the scope of this study, the rating can be used to determine outflow from Northeast Creek. 
The stream stage data collected at station 101 were used to estimate the magnitude of the tidal signal during periods of tidal inflow. Further upstream, stage data collected at station 103 were used to determine the relative change in stage caused by tidal changes or upland freshwater runoff. The continuous temperature and specific conductance data collected at all sites were used to determine the extent and timing of saltwater intrusion upstream into Northeast Creek, or freshwater signatures from surface water or ground water inputs.

\section{Summary of Water Quality and Hydrologic Data}

Although the Northeast Creek estuary/watershed is primarily a freshwater system, it frequently is affected by seawater, which was detected at the most upstream data collection sites during reduced streamflow conditions and high spring tides. This often is observed as a seasonal phenomenon. The increased salinity, which can be determined from the specific conductance (Hem, 1992) was especially evident in bottom waters because of density stratification. Specific conductance (salinity) varied widely at most sites because of tidal influences and freshwater inputs to the estuary/watershed (figs. 2 and 3). Overall specific conductivity in the estuary ranged from less than $100 \mu \mathrm{S} / \mathrm{cm}$ to that of seawater (about 50,000 $\mu \mathrm{S} / \mathrm{cm} ; \mathrm{Hem}$, 1992). Other water-quality constituents, including nutrients and chlorophyll- $a$, exhibited seasonal distribution patterns, with maximum concentrations generally observed in early to midsummer, and again in the fall during both study seasons (fig. 4, and 5).

Results of the water-quality analyses, including associated measurements and applicable quality-assurance data are presented in appendix 1, tables A1-A14. The 15-minute temperature and specific conductance data collected at stations 101-105 are presented as daily values in appendix 2, tables A15-A24. Missing record in the tables is the result of instrument malfunctions that occurred during the study period. The 15-minute stream stage data collected at stations 101 and 103 are presented as daily values in appendix 3, tables A25-A28. The stream stage-discharge relation developed for station 101 is shown in appendix 4, table A29.

\section{May-November 2000}

Dissolved inorganic nitrogen (DIN) species concentrations (nitrate and ammonia) generally were below the reporting limit for nitrate $(0.01 \mathrm{mg} / \mathrm{L})$, and at or below the reporting limit for ammonia $(0.05 \mathrm{mg} / \mathrm{L})$. Some elevated ammonia concentrations $(0.06-0.6 \mathrm{mg} / \mathrm{L})$ however, were detected at the downstream sites (stations 100-103, fig. 4), with the greatest values gener- ally occurring at the most seaward site (station 100). Total nitrogen concentrations varied from 0.26 to $0.82 \mathrm{mg} / \mathrm{L}$ over the entire creek during the open-water season (fig. 4). Peak concentrations were observed in June-July at stations 102-105 (0.68 to $0.77 \mathrm{mg} / \mathrm{L}$ ), with a second peak occurring in September at stations $101-103$ (0.77 to $0.82 \mathrm{mg} / \mathrm{L}$ ). In general, dissolved organic nitrogen (DON) constituted the bulk of the total nitrogen pool.

Phosphorus concentrations showed similar seasonal distributions to those observed for total nitrogen (fig. 4). Reactive (ortho) phosphorus concentrations (reporting limit $=0.1 \mathrm{mg} / \mathrm{L}$ ) generally were relatively low at all sites $(0.1$ to $10.0 \mathrm{mg} / \mathrm{L})$, except for station 100 , the most seaward site, which ranged from 0.5 to $57 \mathrm{mg} / \mathrm{L}$. With the exception of station 100, dissolved inorganic phosphorus contributed little to the total phosphorus pool in the water column. Peaks in total phosphorous (30 to $43 \mathrm{mg} / \mathrm{L}$ ) were observed in late-June at stations 101-105. A second peak ( 27 to $39 \mathrm{mg} / \mathrm{L}$ ) was observed at stations 101-105 between early-September and early-October (fig. 4; appendix 1, tables A2-A12). In both observed seasonal peaks the bulk of the phosphorus occurred in the particulate (organic plus inorganic) form, and/or the dissolved organic form, although the amount within each of these pools was not determined. Total phosphorus at station 100 ranged from 21 to $81 \mathrm{mg} / \mathrm{L}$ during the entire season; ortho-phosphate accounted for 24 to 74 percent of the total phosphorous pool (appendix 1, table A1).

Chlorophyll- $a$ concentrations showed a similar seasonal distribution to that observed for total nitrogen and total phosphorus. A mid-June to early-July peak (18.2-34.9 $\mu \mathrm{g} / \mathrm{L})$ was observed at stations 101-105, with the greatest value $(34.9 \mu \mathrm{g} / \mathrm{L})$ occurring at station 103, Aunt Betsy's Brook (fig. 4). A second lower peak (8.8-12.9 $\mu \mathrm{g} / \mathrm{L})$ was observed in early-October at stations 101-103, with the greatest value again recorded at station 103 (fig. 4). Chlorophyll- $a$ concentrations at station 100 , the most seaward site, were relatively low (0.8$3.4 \mu \mathrm{g} / \mathrm{L}$ ) during the entire season. Note that chlorophyll- $a$ concentrations shown in figure 4 are plotted as total chlorophyll (chlorophyll- $a$ plus pheophytin), and are slightly higher than the concentrations shown in appendix 1 , which were corrected for pheophytin.

The period of continuous water temperature and specific conductivity record collected at the individual stations varied because of different equipment installation dates and periods of missing record. For May through November 2000 (the period of biweekly sampling) the minimum recorded water temperature was $0.2^{\circ} \mathrm{C}$ at station 105 on November 25 and the maximum was $31^{\circ} \mathrm{C}$ at station 103 on August 11. During the same period, the minimum specific conductance was $16 \mu \mathrm{S} / \mathrm{cm}$ on May 21 at station 104 and the maximum was 53,500 $\mu \mathrm{S} / \mathrm{cm}$ on October 28 at station 101 . 

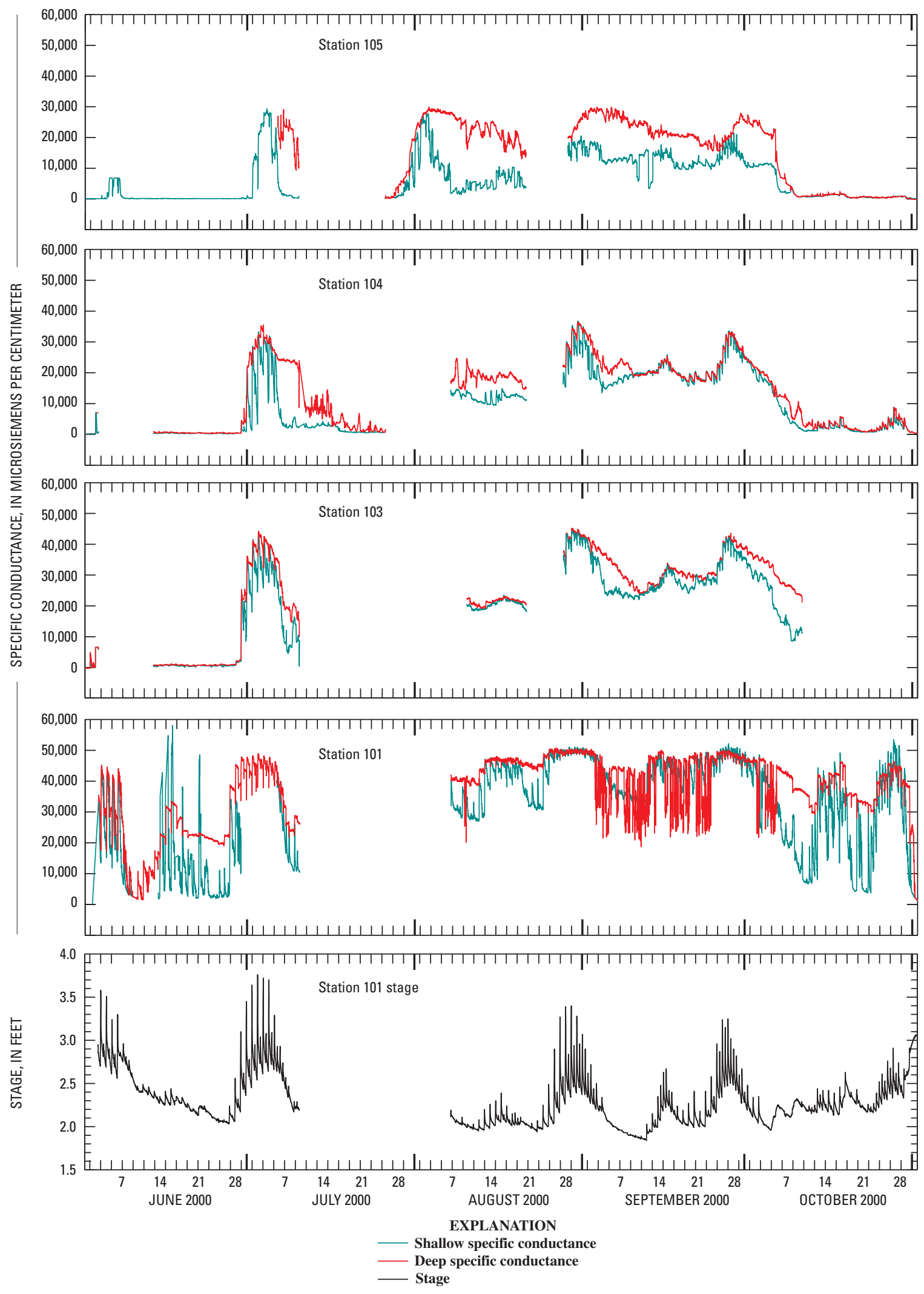

Figure 2. Continuous specific conductance and stage at selected sites during June-0ctober 2000 in the Northeast Creek-Fresh Meadow estuary, Acadia National Park, Maine. 

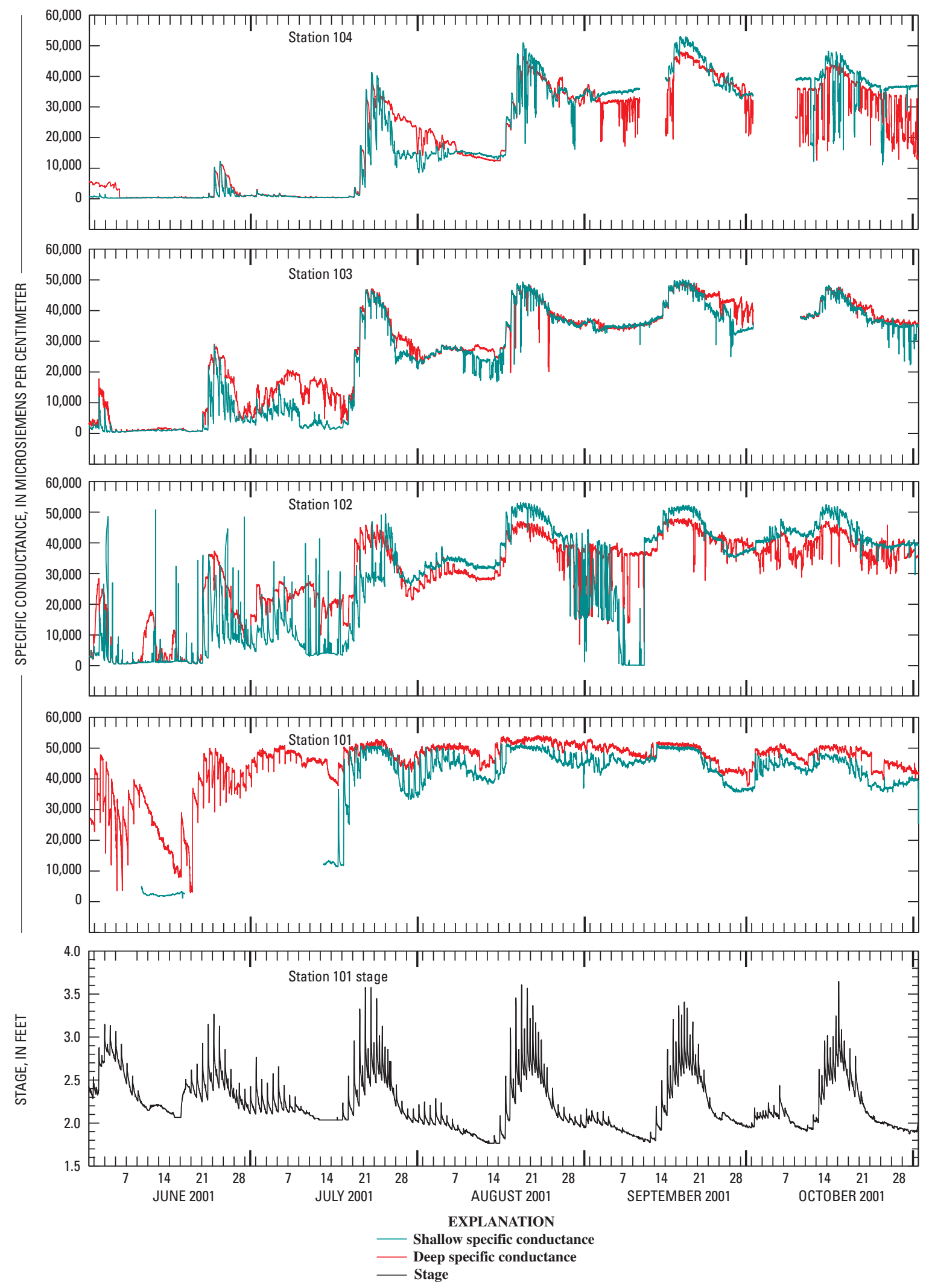

Figure 3. Continuous specific conductance and stage at selected sites during June-0ctober 2001 in the Northeast Creek-Fresh Meadow estuary, Acadia National Park, Maine. 
Total nitrogen
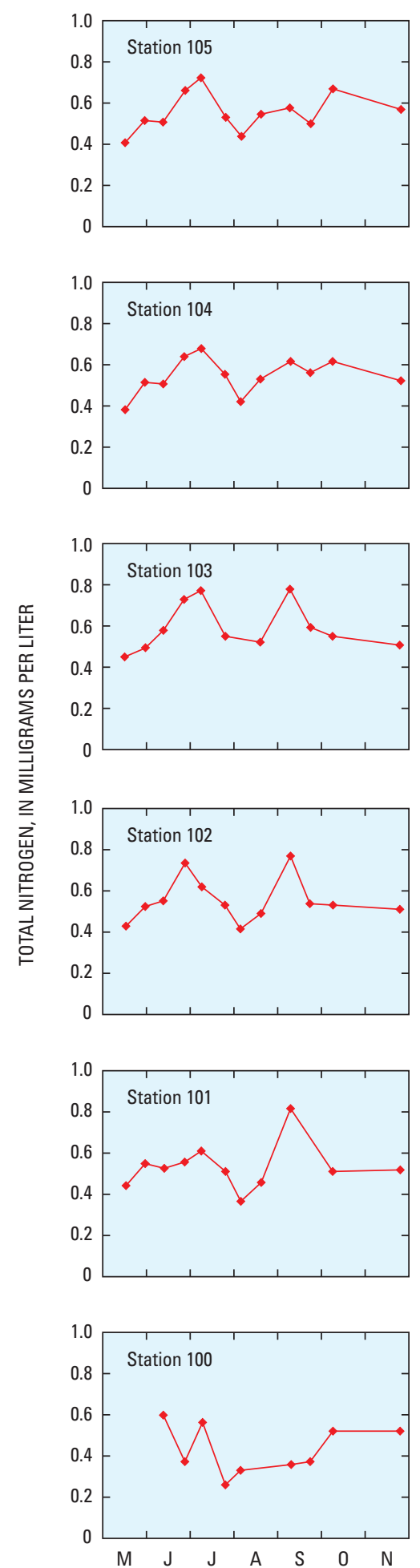

Total phosphorus
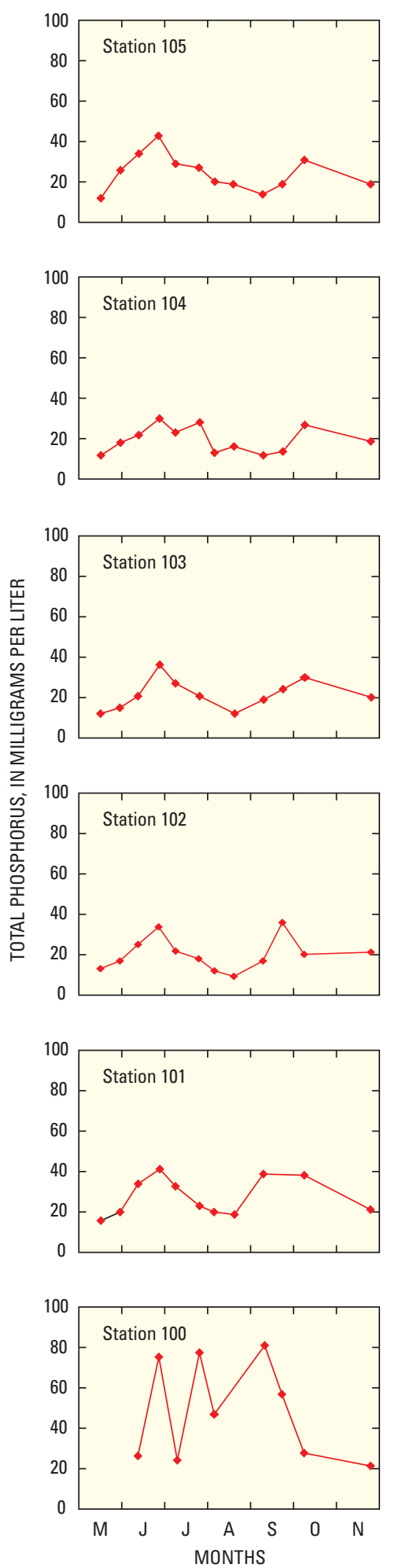

Total chlorophyll-a
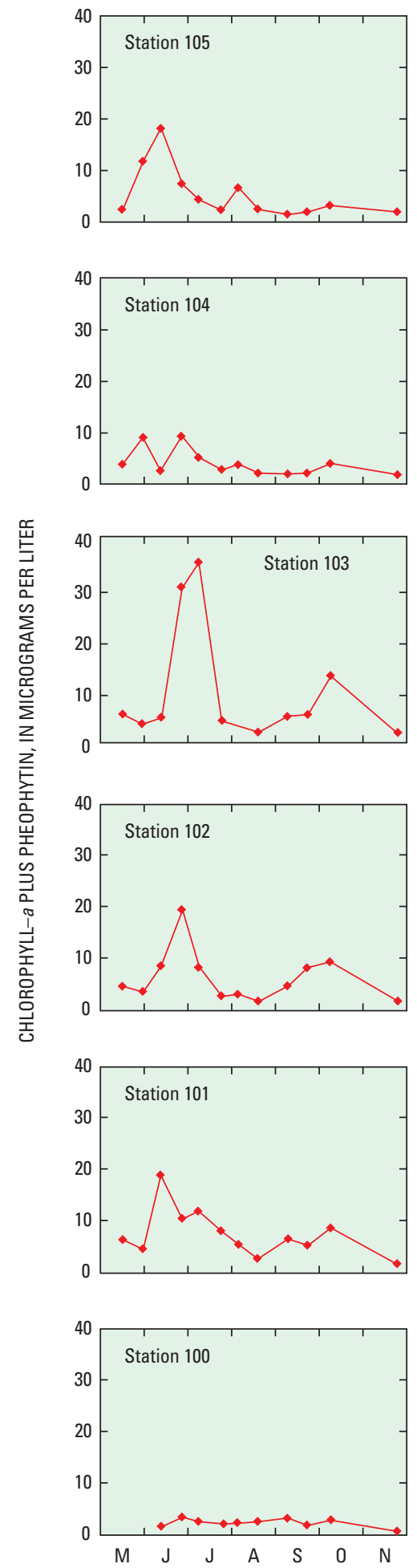

Figure 4. Total nitrogen, phosphorus, and chlorophyll-a concentrations during May-November 2000 at stations 100-105 in the Northeast Creek-Fresh Meadow estuary, Acadia National Park, Maine. 

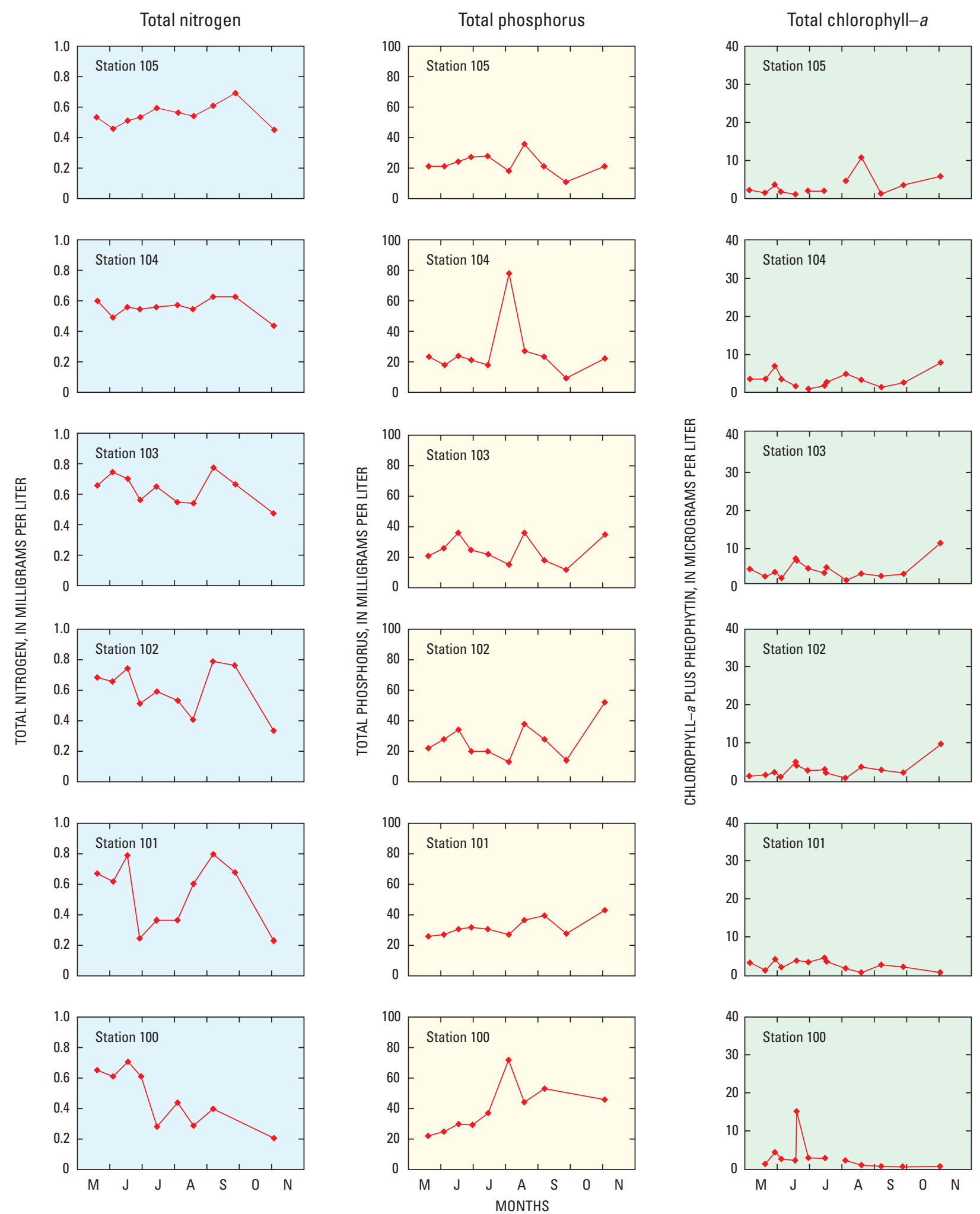

Figure 5. Total nitrogen, phosphorus, and chlorophyll-a concentrations during May-November 2001 at stations 100-105 in the Northeast Creek-Fresh Meadow estuary, Acadia National Park, Maine. 


\section{May-November 2001}

The same general trends observed in the 2000 field season occurred in 2001, although the maxima occurred slightly earlier in 2001. DIN concentrations (nitrate, nitrite and ammonia) were generally below the reporting limit for nitrate and nitrite $(0.01 \mathrm{mg} / \mathrm{L})$, and at or below the reporting limit for ammonia $(0.05 \mathrm{mg} / \mathrm{L})$. As in 2000 however, some elevated ammonia concentrations $(0.1-0.5 \mathrm{mg} / \mathrm{L})$ were detected at the downstream sites (stations 100-103), with the greatest values generally occurring at the most seaward site (station 100). Total nitrogen concentrations ranged from 0.21 to $0.80 \mathrm{mg} / \mathrm{L}$ over the entire creek during the open water season (fig. 5). Peak concentrations ( 0.71 to $0.79 \mathrm{mg} / \mathrm{L}$ ) were observed at stations $100-103$ in early to mid-June, with a second peak ( 0.39 to $0.80 \mathrm{mg} / \mathrm{L})$ occurring at all sites in late-August through late-September. Although a second peak was detected at all sites, the greatest values ( 0.78 to $0.80 \mathrm{mg} / \mathrm{L}$ ) occurred at stations $101-103$. As in the 2000 field season, dissolved organic nitrogen (DON) constituted the bulk of the total nitrogen pool.

Phosphorus concentrations showed similar seasonal distributions to those observed in the 2000 field season, although the fall maxima occurred slightly later in the season (fig. 5). Reactive (ortho) phosphorus concentrations were low ( 1.4 to $11 \mathrm{mg} / \mathrm{L}$ ) at stations $103-105$, compared to the more seaward stations 100-102, where concentrations ranged from 4.3 to $39 \mathrm{mg} / \mathrm{L}$. As in the 2000 field season, ortho-phosphate accounted for a substantial portion of the total phosphorus pool at station 100. With the exception of station 101 during the spring, dissolved inorganic phosphorus contributed little to the total phosphorus pool in the water column at the upstream sites. As in 2000, most of the total phosphorus occurred in the particulate (organic plus inorganic) form, and/or the dissolved organic form, although the amount within each of these pools was not determined. Maximum concentrations were observed in mid-June (24 to $36 \mathrm{mg} / \mathrm{L}$ ), August ( 36 to $78 \mathrm{mg} / \mathrm{L}$ ), and earlyNovember ( 21 to $52 \mathrm{mg} / \mathrm{L}$ ). Total phosphorus at station 100 ranged from 22 to $72 \mathrm{mg} / \mathrm{L}$ over the entire season, with the maximum occurring in early-August; ortho-phosphate accounted for 17 to 74 percent of the total phosphorous pool (fig. 5; appendix 1, table A7).

Chlorophyll- $a$ concentrations also showed a similar seasonal distribution to that observed in 2000 , with small peaks occurring in mid-June ( 8.1 to $24.1 \mu \mathrm{g} / \mathrm{L}$ ), and larger peaks (6.7 to $14.7 \mu \mathrm{g} / \mathrm{L}$ ), associated with stations 102-105, occurring in early-November. The greatest concentration $(14.7 \mu \mathrm{g} / \mathrm{L})$ was observed at station 103, Aunt Betsy's Brook. As in 2000, the chlorophyll- $a$ concentrations at the most seaward site (station 100 ), were low ( 2.3 to $7.7 \mu \mathrm{g} / \mathrm{L}$ ) during the entire season, with the exception of one sample in mid-June that had a concentration of $24.1 \mu \mathrm{g} / \mathrm{L}$ (fig. 5; appendix 1, table A7).

During the 2001 field season, the period of continuous water temperature and specific conductivity record collected at the individual stations varied because of different equipment installation dates and periods of missing record. For May through November 2001 (the period of biweekly sampling) the minimum recorded water temperature was $2.7^{\circ} \mathrm{C}$ at station 102 on October 31 , and the maximum was $34.5^{\circ} \mathrm{C}$ at station 101 on June 15. During the same period at station 101, the minimum recorded specific conductance was $84 \mu \mathrm{S} / \mathrm{cm}$ on June 5 and the maximum was $53,900 \mu \mathrm{S} / \mathrm{cm}$ on June 9 .

\section{References Cited}

Acadia National Park, 2000, Water Resources Management Plan: NPS D-209, April 2000, 102 p.

American Public Health Association, American Water Works Association, and Water Pollution Control Federation, 1998, Standard methods for the examination of water and wastewater (20th ed.): Washington, D.C., American Public Health Association [variously paged].

Carter, R.W., and Davidian, J., 1968, General procedure for gaging streams: U.S. Geological Survey Techniques of Water-Resources Investigations, book 3, chap. A6, 13 p.

Doering, P.H., Beatty, L.L., Keller, A.A., Oviatt, C.A., and Roman, C.T., 1995, Water quality and habitat evaluation of Bass Harbor Marsh, Acadia National Park, Maine: National Park Service Technical Report: NPS/NESORNR/NRTR/ 95-31, $148 \mathrm{p}$.

Hem, J.D., 1992, Study and interpretation of the chemical characteristics of natural water, U.S. Geological Survey WaterSupply Paper 2254, 263 p.

Holm-Hansen, O., Lorenzen, C.J., Holmes, R.W., and Strickand, J.D.H., 1965, Fluorometric determination of chlorophyll: Journal du Conseil International pour l'Exploration de la Mer., no. 30, p. 3-15.

Long, H.K., Daddow, R.L., and Farrar, J.W., 1998, U.S. Geological Survey standard reference sample project: Performance evaluation of analytical laboratories: U.S. Geological Survey Fact Sheet FS-031-98, 4 p.

Lorenzen, C.J., 1967, Determination of chlorophyll and phaeopigments: Spectrophotometric Equations: Limnology and Oceanography, no. 12, p. 343-346.

Manski, D., 1998, Resource Management Plan for Acadia National Park, 33 p.

Morrison, M., 1989, Quality-assurance plan for the long-term monitoring program: U.S. Environmental Protection Agency Laboratory, Chervils, Oregon, 77 p.

National Oceanic and Atmospheric Administration, 19791999, Climatological Data Annual Summaries.

Neckles, H.A., Kopp, B.S., and Guntenspergen, G.R., 2003, Autotrophic responses to nutrient loadings in a Ruppia-dominated estuary: Current status and future projections, 17th Biennial Conference of the Estuarine Research Federation, September 14-18, Seattle, Washington.

Nielsen, J.P., Stewart, G.J., and Caldwell, J.M., 2000, Water resources data for Maine, water year 1999: U.S. Geological Survey Water-Data Report ME-99-1, 194 p.

Nielsen, M.G., 2002, Water budget for and nitrogen loads to Northeast Creek, Bar Harbor, Maine: U.S. Geological 
Survey Water Resources Investigations Report 02-4000, $32 \mathrm{p}$.

Rantz, S.E., and others, 1982, Measurement and computation of streamflow: U.S. Geological Survey Water-Supply Paper 2175, v. 2, 631 p.

Stewart, G.J., Nielsen, J.P., Caldwell, J.M., and Cloutier, A.R., 2001, Water resources data for Maine, water year 2000: U.S. Geological Survey Water-Data Report ME-00-01, 233 p.

U.S. Census Bureau, 2001, American housing survey generated by Martha Nielsen using data extraction system:

http://www.census.gov/des/p1; (21 November 2001).
U.S. Environmental Protection Agency, 1979, Methods for chemical analysis of water and wastes: EPA 600/4-79-020, 460 p. Revised 1983.

U.S. Environmental Protection Agency, 1984, Method 300.0, Determination of inorganic anions in drinking water by ion chromatograph: EPA 600/4-84-017, Revised 1993.

U.S. Environmental Protection Agency, 1987, Handbook of methods for acid deposition studies: laboratory analysis for surface water chemistry: EPA 600/4-87-026 [variously paged].

U.S. Environmental Protection Agency, 1993, Methods for the determination of inorganic substances in environmental samples: EPA 600/R-93-100, 169 p. 


\section{Appendix 1}

Bi-weekly water-quality data, all stations 
Table A1. Water-quality data collected May-November 2000 at station 100 (USGS identifier 442530068193901) Northeast Creek below Route 3 bridge near Bar Harbor, ME [Numbers in parentheses are USGS NWIS parameter code numbers. Sampling depths are relative to the water surface. Abbreviations: A, average value; deg C, degrees Celsius; fltrd, filtered; mg/L, milligrams per liter; $\mathrm{mm} \mathrm{Hg}$, millimeters of mercury; N, nitrogen; P, phosphorus; std, standard; ug/L, micrograms per liter; unfltrd, unfiltered; uS/cm, microsiemens per centimeter; wat unf, unfiltered water; <, less than; --, missing data]. Chlorophyll-a results have been corrected for pheophytin and are reported as the mean of triplicate subsamples from a composited sample collected at each sampling date.

\begin{tabular}{|c|c|c|c|c|c|c|c|c|}
\hline Date & Time & $\begin{array}{c}\text { Sam- } \\
\text { pling } \\
\text { depth, } \\
\text { meters } \\
\text { (00098) }\end{array}$ & $\begin{array}{l}\text { Baro- } \\
\text { metric } \\
\text { pres- } \\
\text { sure, } \\
\text { mm Hg } \\
(00025)\end{array}$ & $\begin{array}{c}\text { Dis- } \\
\text { solved } \\
\text { oxygen, } \\
\text { mg/L } \\
(00300)\end{array}$ & $\begin{array}{c}\text { Dis- } \\
\text { solved } \\
\text { oxygen, } \\
\text { percent } \\
\text { of sat- } \\
\text { uration } \\
\text { (00301) }\end{array}$ & $\begin{array}{c}\text { pH, } \\
\text { water, } \\
\text { unfltrd } \\
\text { field, } \\
\text { std } \\
\text { units } \\
(00400)\end{array}$ & $\begin{array}{c}\text { Specif. } \\
\text { conduc- } \\
\text { tance, } \\
\text { wat unf } \\
\text { uS/cm } \\
25 \text { degc } \\
(00095)\end{array}$ & $\begin{array}{l}\text { Temper- } \\
\text { ature, } \\
\text { water, } \\
\text { deg C } \\
(00010)\end{array}$ \\
\hline \multicolumn{9}{|l|}{ JUN 2000} \\
\hline $\begin{array}{l}14 \ldots \\
28 \ldots\end{array}$ & $\begin{array}{l}1020 \\
0800\end{array}$ & $\begin{array}{l}.30 \\
.10\end{array}$ & $\begin{array}{l}757 \\
756\end{array}$ & $\begin{array}{l}9.3 \\
5.9\end{array}$ & $\begin{array}{r}103 \\
73\end{array}$ & $\begin{array}{l}7.8 \\
7.3\end{array}$ & $\begin{array}{l}32400 \\
42800\end{array}$ & $\begin{array}{l}13.9 \\
17.0\end{array}$ \\
\hline \multicolumn{9}{|l|}{ JUL } \\
\hline $\begin{array}{l}11 \ldots \\
27 \ldots\end{array}$ & $\begin{array}{l}0815 \\
0750\end{array}$ & $\begin{array}{l}.10 \\
.20\end{array}$ & $\begin{array}{l}754 \\
772\end{array}$ & $\begin{array}{l}4.4 \\
4.3\end{array}$ & $\begin{array}{l}52 \\
53\end{array}$ & $\begin{array}{l}7.1 \\
7.3\end{array}$ & $\begin{array}{l}36800 \\
47000\end{array}$ & $\begin{array}{l}16.4 \\
16.8\end{array}$ \\
\hline \multicolumn{9}{|l|}{ AUG } \\
\hline $\begin{array}{l}07 \ldots \\
22 \ldots\end{array}$ & $\begin{array}{l}1740 \\
1625\end{array}$ & $\begin{array}{l}.30 \\
.30\end{array}$ & $\begin{array}{l}758 \\
770\end{array}$ & $\begin{array}{r}7.0 \\
10.6\end{array}$ & $\begin{array}{r}90 \\
151\end{array}$ & $\begin{array}{l}7.6 \\
8.1\end{array}$ & $\begin{array}{l}46300 \\
47800\end{array}$ & $\begin{array}{l}18.6 \\
24.6\end{array}$ \\
\hline \multicolumn{9}{|l|}{ SEP } \\
\hline $\begin{array}{l}12 \ldots \\
12 \ldots \\
25 \ldots\end{array}$ & $\begin{array}{l}1100 \\
1115 \\
1015\end{array}$ & $\begin{array}{l}.60 \\
.50 \\
.30\end{array}$ & $\begin{array}{l}752 \\
752 \\
761\end{array}$ & $\begin{array}{r}11.6 \\
10.6 \\
6.6\end{array}$ & $\begin{array}{r}148 \\
137 \\
76\end{array}$ & $\begin{array}{l}7.8 \\
7.8 \\
7.5\end{array}$ & $\begin{array}{l}50900 \\
50900 \\
50100\end{array}$ & $\begin{array}{l}16.7 \\
17.2 \\
12.1\end{array}$ \\
\hline \multicolumn{9}{|l|}{ OCT } \\
\hline$\underset{\text { NOV }}{11} \ldots$ & 1115 & .40 & 754 & 9.9 & 98 & 7.5 & 41700 & 6.9 \\
\hline $29 \ldots$ & 1245 & .20 & 758 & 9.1 & 69 & 6.0 & 157 & 3.3 \\
\hline Date & Time & $\begin{array}{c}\text { Calcium } \\
\text { water, } \\
\text { fltrd, } \\
\text { mg/L } \\
(00915)\end{array}$ & $\begin{array}{c}\text { Magnes- } \\
\text { ium, } \\
\text { water, } \\
\text { fltrd, } \\
\text { mg/L } \\
(00925)\end{array}$ & $\begin{array}{c}\text { Potas- } \\
\text { sium, } \\
\text { water, } \\
\text { fltrd, } \\
\text { mg/L } \\
\text { (00935) }\end{array}$ & $\begin{array}{c}\text { Sodium, } \\
\text { water, } \\
\text { fltrd, } \\
\text { mg/L } \\
(00930)\end{array}$ & $\begin{array}{c}\text { Chlor- } \\
\text { ide, } \\
\text { water, } \\
\text { fltrd, } \\
\text { mg/L } \\
(00940)\end{array}$ & $\begin{array}{c}\text { Silica, } \\
\text { water, } \\
\text { fltrd, } \\
\text { mg/L } \\
(00955)\end{array}$ & $\begin{array}{c}\text { Sulfate } \\
\text { water, } \\
\text { fltrd, } \\
\text { mg/L } \\
\text { (00945) }\end{array}$ \\
\hline \multicolumn{9}{|l|}{ JUN 2000} \\
\hline $\begin{array}{l}14 \ldots \\
28 \ldots\end{array}$ & $\begin{array}{l}1030 \\
0755\end{array}$ & $\begin{array}{l}42.0 \\
255\end{array}$ & $\begin{array}{l}142 \\
780\end{array}$ & $\begin{array}{l}38.5 \\
251\end{array}$ & $\begin{array}{r}954 \\
6230\end{array}$ & $\begin{array}{l}2040 \\
3000\end{array}$ & $\begin{array}{l}.480 \\
.290\end{array}$ & $\begin{array}{r}267 \\
1660\end{array}$ \\
\hline \multicolumn{9}{|l|}{ JUL } \\
\hline $\begin{array}{l}11 \ldots \\
27 \ldots\end{array}$ & $\begin{array}{l}0800 \\
0745\end{array}$ & $\begin{array}{l}127 \\
331\end{array}$ & $\begin{array}{l}388 \\
957\end{array}$ & $\begin{array}{l}131 \\
309\end{array}$ & $\begin{array}{l}3390 \\
8250\end{array}$ & $\begin{array}{r}5280 \\
15300\end{array}$ & $\begin{array}{l}.360 \\
.240\end{array}$ & $\begin{array}{r}768 \\
1870\end{array}$ \\
\hline \multicolumn{9}{|l|}{ AUG } \\
\hline $\begin{array}{r}07 \ldots \\
22 \ldots\end{array}$ & $\begin{array}{l}1730 \\
1620\end{array}$ & $\begin{array}{l}285 \\
339\end{array}$ & $\begin{array}{r}852 \\
1020\end{array}$ & $\begin{array}{l}302 \\
333\end{array}$ & $\begin{array}{l}7640 \\
9500\end{array}$ & $\begin{array}{l}24200 \\
17900\end{array}$ & $\begin{array}{l}.200 \\
.320\end{array}$ & $\begin{array}{l}4730 \\
2520\end{array}$ \\
\hline $\begin{array}{l}\mathrm{SEP} \\
\quad 12 \ldots \\
25 \ldots\end{array}$ & $\begin{array}{l}1050 \\
1010\end{array}$ & $\begin{array}{l}373 \\
342\end{array}$ & $\begin{array}{l}1150 \\
1050\end{array}$ & $\begin{array}{l}395 \\
381\end{array}$ & $\begin{array}{l}9190 \\
8610\end{array}$ & $\begin{array}{l}26900 \\
16700\end{array}$ & $\begin{array}{l}.230 \\
.160\end{array}$ & $\begin{array}{l}2580 \\
2320\end{array}$ \\
\hline \multicolumn{9}{|l|}{$\mathrm{OCT}$} \\
\hline$\underset{N O V}{11 \ldots}$ & 1110 & 116 & 286 & 115 & 2280 & 4580 & 1.53 & 619 \\
\hline $29 \ldots$ & 1245 & 2.73 & 2.29 & 1.61 & 22.0 & 30.0 & 2.66 & 7.0 \\
\hline
\end{tabular}


Table A1. Water-quality data collected May-November 2000 at station 100 (USGS identifier 442530068193901) Northeast Creek below Route 3 bridge near Bar Harbor, ME - Continued.

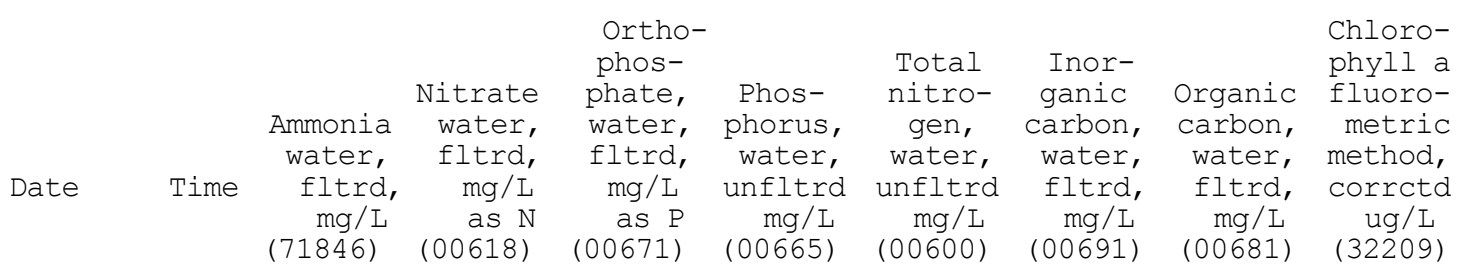

\begin{tabular}{|c|c|c|c|c|c|c|c|c|c|}
\hline \multicolumn{10}{|c|}{ JUN 2000} \\
\hline $\begin{array}{l}14 \ldots \\
28 \ldots\end{array}$ & $\begin{array}{l}1030 \\
0755\end{array}$ & $\begin{array}{l}.04 \\
.14\end{array}$ & $\begin{array}{l}.00 \\
.00\end{array}$ & $\begin{array}{r}8.10 \\
49.0\end{array}$ & $\begin{array}{l}26.0 \\
75.0\end{array}$ & $\begin{array}{l}.60 \\
.37\end{array}$ & $\begin{array}{r}5.6 \\
24.0\end{array}$ & $\begin{array}{r}11.6 \\
3.7\end{array}$ & $\begin{array}{l}\text { A1. } 50 \\
\text { A3.30 }\end{array}$ \\
\hline \multicolumn{10}{|l|}{ JUL } \\
\hline $11 \ldots$ & 0800 & .03 & .00 & .000 & 24.0 & .56 & 11.0 & 6.2 & A2. 50 \\
\hline $27 \ldots$ & 0745 & .15 & .00 & 57.0 & 77.0 & .26 & -- & 1.2 & A2. 00 \\
\hline \multicolumn{10}{|l|}{ AUG } \\
\hline $07 \ldots$ & 1730 & .25 & .00 & 21.0 & 47.0 & .33 & 16.1 & 1.3 & A2. 60 \\
\hline $22 \ldots$ & 1620 & .52 & .00 & 51.0 & -- & .35 & 17.7 & 1.0 & A2. 50 \\
\hline \multicolumn{10}{|l|}{ SEP } \\
\hline $12 \ldots$ & 1050 & .60 & .00 & 49.0 & 81.0 & .36 & 25.2 & .6 & A3. 20 \\
\hline $25 \ldots$ & 1010 & .54 & .00 & 33.0 & 57.0 & .37 & 24.0 & .8 & A1. 70 \\
\hline \multicolumn{10}{|l|}{ OCT } \\
\hline $11 \ldots$ & 1110 & .03 & .00 & 9.10 & 28.0 & .52 & 8.2 & 4.0 & A2. 70 \\
\hline $\begin{array}{l}\text { NOV } \\
29\end{array}$ & 1245 & 03 & مी & 50 & 210 & 52 & 23 & 110 & \\
\hline & 1245 & .03 & .00 & 5.00 & 21.0 & .52 & 2.3 & 14.9 & A. \\
\hline
\end{tabular}


Table A2. Water-quality data collected May-November 2000 at station 101 (USGS identifier 01022820) Northeast Creek at Route 3 bridge near Bar Harbor, ME [Numbers in parentheses are USGS NWIS parameter code numbers. Sampling depths are relative to the water surface. Abbreviations: A, average value; deg C, degrees Celsius; fltrd, filtered; mg/L, milligrams per liter; mm $\mathrm{Hg}$, millimeters of mercury; $\mathrm{N}$, nitrogen; P, phosphorus; std, standard; ug/L, micrograms per liter; unfltrd, unfiltered; uS/cm, microsiemens per centimeter; wat unf, unfiltered water; <, less than; --, missing data]. Chlorophyll-a results have been corrected for pheophytin and are reported as the mean of triplicate subsamples from a composited sample collected at each sampling date.

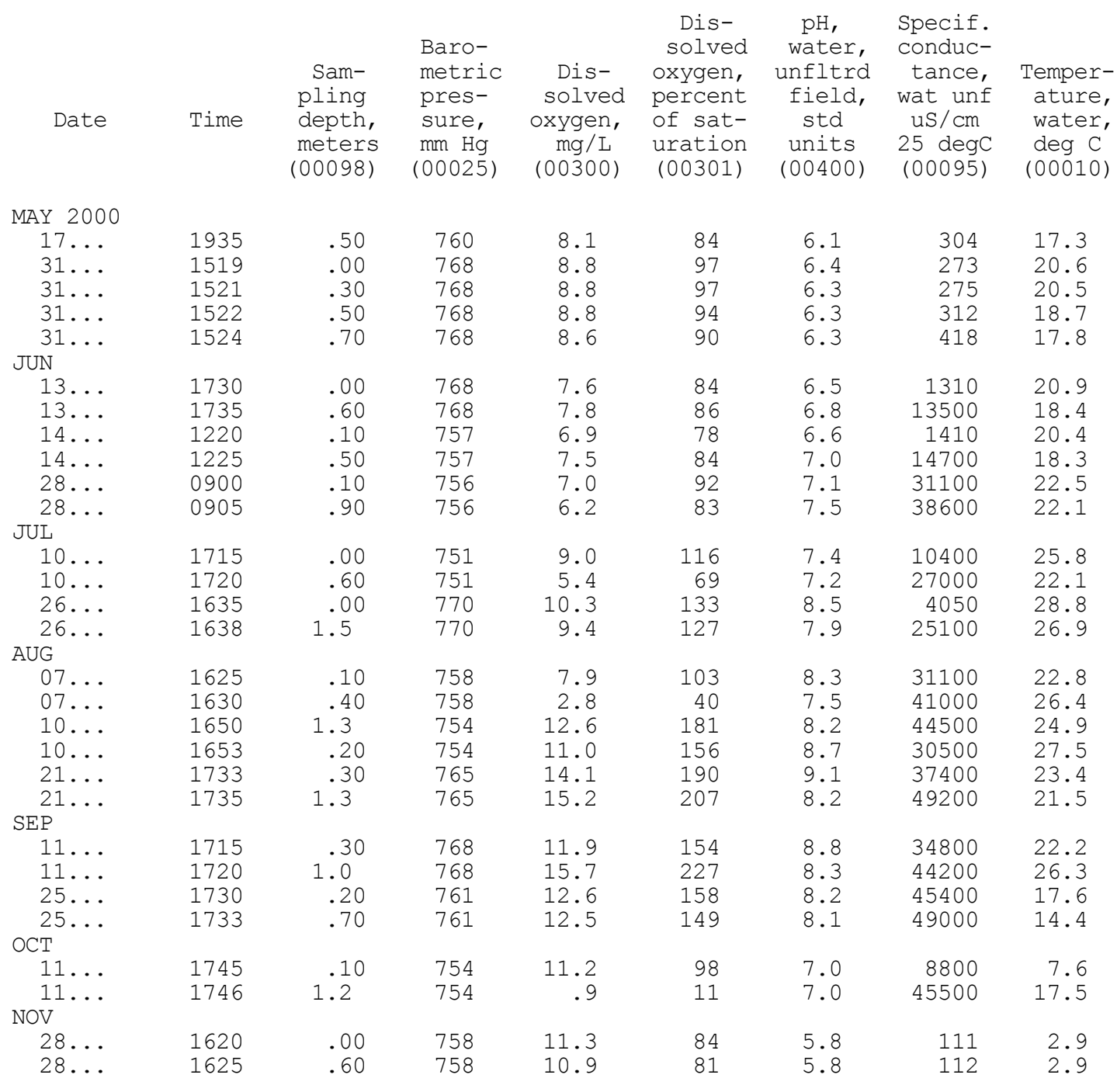


Table A2. Water-quality data collected May-November 2000 at station 101 (USGS identifier 01022820) Northeast Creek at Route 3 bridge near Bar Harbor, ME - Continued.

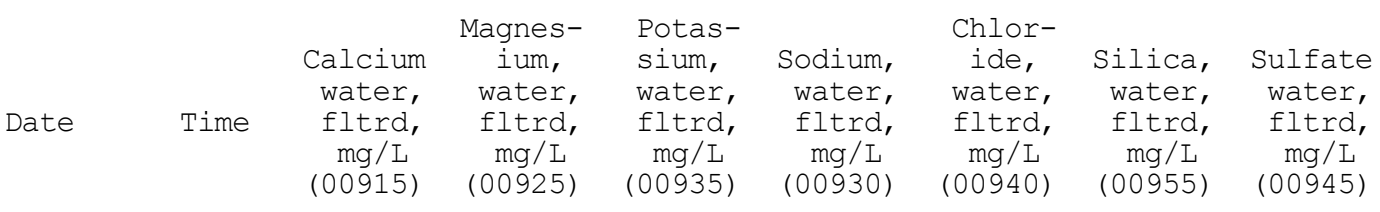

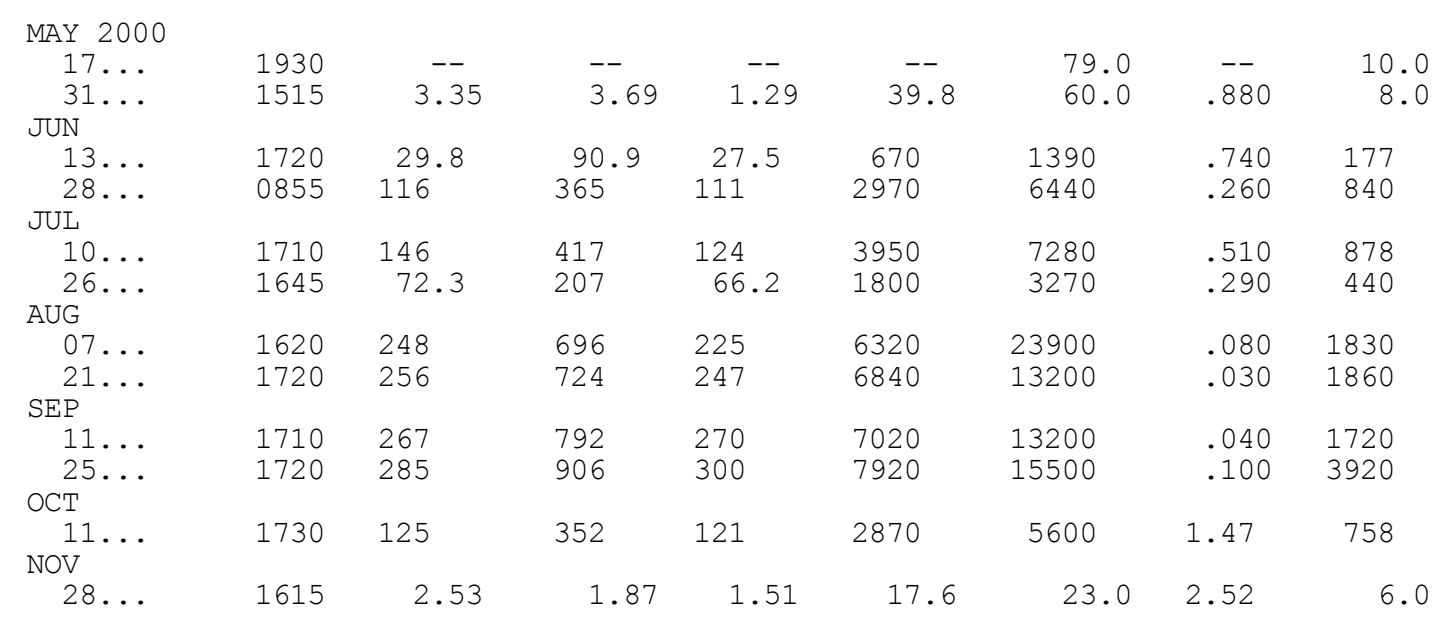

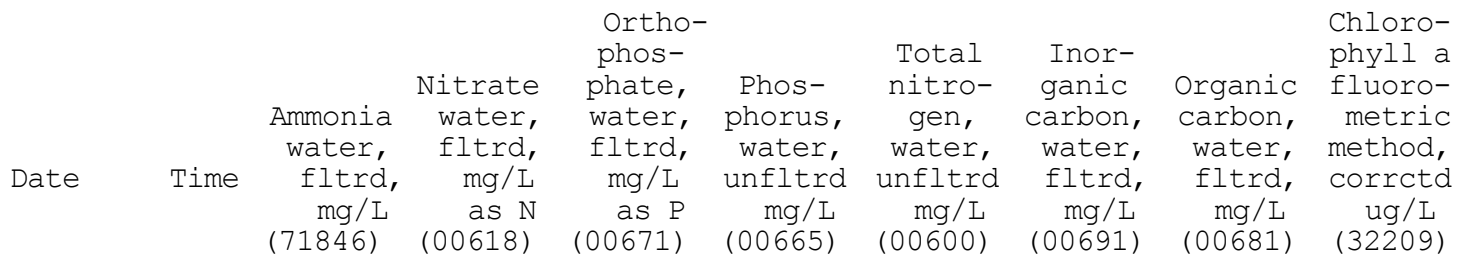

\begin{tabular}{|c|c|c|c|c|c|c|c|c|c|}
\hline \multicolumn{10}{|c|}{ MAY 2000} \\
\hline $17 \ldots$ & 1930 & -- & .00 & -- & 16.0 & .44 & -- & 12.8 & A2.10 \\
\hline $31 \ldots$ & 1515 & .01 & .00 & 3.00 & 20.0 & .55 & 2.9 & 13.4 & A1. 30 \\
\hline \multicolumn{10}{|l|}{ JUN } \\
\hline $13 \ldots$ & 1720 & .03 & .00 & 9.90 & 34.0 & .53 & 5.7 & 13.0 & A10.0 \\
\hline $28 \ldots$ & 0855 & .02 & .00 & 9.20 & 41.0 & .56 & 12.0 & 7.9 & A5. 00 \\
\hline \multicolumn{10}{|l|}{ JUL } \\
\hline $10 \ldots$ & 1710 & .02 & .00 & .000 & 33.0 & .61 & 11.0 & 6.0 & A6.30 \\
\hline $26 \ldots$ & 1645 & .01 & .00 & 5.10 & 23.0 & .52 & -- & 5.0 & A5.00 \\
\hline \multicolumn{10}{|l|}{ AUG } \\
\hline $07 \ldots$ & 1620 & .15 & .00 & 4.50 & 20.0 & .36 & 11.5 & 1.7 & A3. 40 \\
\hline $21 \ldots$ & 1720 & .16 & .00 & 2.00 & 19.0 & .46 & 11.2 & 1.7 & A2.00 \\
\hline \multicolumn{10}{|l|}{ SEP } \\
\hline $11 \ldots$ & 1710 & .18 & .00 & 6.00 & 39.0 & .82 & 10.7 & 2.5 & A 3.80 \\
\hline $25 \ldots$ & 1720 & .45 & .00 & 13.0 & -- & .32 & 20.0 & 1.0 & A2. 80 \\
\hline \multicolumn{10}{|l|}{ OCT } \\
\hline $11 \ldots$ & 1730 & .02 & .00 & 8.40 & 38.0 & .51 & 9.0 & 3.7 & A4. 80 \\
\hline \multicolumn{10}{|l|}{ NOV } \\
\hline $28 \ldots$ & 1615 & .02 & .00 & 4.20 & 21.0 & .52 & 2.1 & 14.9 & A. 700 \\
\hline
\end{tabular}


Table A3. Water-quality data collected May-November 2000 at station 102 (USGS identifier 442517068190501) Northeast Creek near Bar Harbor, ME [Numbers in parentheses are USGS NWIS parameter code numbers. Sampling depths are relative to the water surface. Abbreviations: A, average value; deg C, degrees Celsius; fltrd, filtered; $\mathrm{mg} / \mathrm{L}$, milligrams per liter; $\mathrm{mm} \mathrm{Hg}$, millimeters of mercury; $\mathrm{N}$, nitrogen; P, phosphorus; std, standard; ug/L, micrograms per liter; unfltrd, unfiltered; uS/cm, microsiemens per centimeter; wat unf, unfiltered water; <, less than; --, missing data]. Chlorophyll-a results have been corrected for pheophytin and are reported as the mean of triplicate subsamples from a composited sample collected at each sampling date.

\begin{tabular}{|c|c|c|c|c|c|c|c|c|}
\hline ate & Time & $\begin{array}{c}\text { Sam- } \\
\text { pling } \\
\text { depth, } \\
\text { meters } \\
(00098)\end{array}$ & $\begin{array}{l}\text { Baro- } \\
\text { metric } \\
\text { pres- } \\
\text { sure, } \\
\text { mm Hg } \\
(00025)\end{array}$ & $\begin{array}{c}\text { Dis- } \\
\text { solved } \\
\text { oxygen, } \\
\text { mg/L } \\
(00300)\end{array}$ & $\begin{array}{c}\text { Dis- } \\
\text { solved } \\
\text { oxygen, } \\
\text { percent } \\
\text { of sat- } \\
\text { uration } \\
\text { (00301) }\end{array}$ & $\begin{array}{c}\text { pH, } \\
\text { water, } \\
\text { unfltrd } \\
\text { field, } \\
\text { std } \\
\text { units } \\
(00400)\end{array}$ & $\begin{array}{c}\text { Specif. } \\
\text { conduc- } \\
\text { tance, } \\
\text { wat unf } \\
\text { uS } / \mathrm{cm} \\
25 \mathrm{deg} C \\
(00095)\end{array}$ & $\begin{array}{c}\text { Temper- } \\
\text { ature, } \\
\text { water, } \\
\text { deg C } \\
(00010)\end{array}$ \\
\hline
\end{tabular}

\begin{tabular}{|c|c|c|c|c|c|c|c|c|}
\hline MAY 2000 & & & & & & & & \\
\hline $17 \ldots$ & 1855 & .50 & 760 & 8.2 & 85 & 6.1 & 195 & 17.4 \\
\hline $31 \ldots$ & 1401 & .10 & 768 & 8.8 & 94 & 6.3 & 286 & 18.5 \\
\hline $31 \ldots$ & 1402 & .30 & 768 & 8.9 & 94 & 6.3 & 284 & 18.5 \\
\hline $31 \ldots$ & 1403 & .60 & 768 & 8.9 & 93 & 6.3 & 287 & 18.1 \\
\hline JUN & & & & & & & & \\
\hline $03 \ldots$ & 1300 & .10 & 760 & 8.1 & 94 & 6.9 & 15700 & 19.5 \\
\hline $03 \ldots$ & 1305 & .40 & 760 & 8.1 & 95 & 7.3 & 38700 & 15.7 \\
\hline $13 \ldots$ & 1700 & .10 & 768 & 7.7 & 84 & 6.4 & 936 & 20.2 \\
\hline $13 \ldots$ & 1705 & .40 & 768 & 7.6 & 84 & 6.4 & 946 & 20.1 \\
\hline $14 \ldots$ & 1155 & .20 & 757 & 6.7 & 73 & 6.3 & 1050 & 19.6 \\
\hline $14 \ldots$ & 1200 & .50 & 757 & 6.9 & 74 & 6.3 & 1060 & 18.4 \\
\hline $28 \ldots$ & 0930 & .00 & 756 & 5.1 & 62 & 6.7 & 1510 & 24.3 \\
\hline $28 \ldots$ & 0935 & .40 & 756 & 4.4 & 52 & 6.6 & 2410 & 22.8 \\
\hline JUL & & & & & & & & \\
\hline $10 \ldots$ & 1615 & .00 & 751 & 8.2 & 103 & 6.7 & 10000 & 24.4 \\
\hline $10 \ldots$ & 1620 & .50 & 751 & 7.3 & 97 & 6.8 & 25300 & 24.3 \\
\hline $26 \ldots$ & 1612 & .00 & 770 & 8.3 & 111 & 6.8 & 3550 & 30.2 \\
\hline $26 \ldots$ & 1613 & .60 & 770 & 8.7 & 108 & 6.8 & 12900 & 24.6 \\
\hline AUG & & & & & & & & \\
\hline $07 \ldots$ & 1555 & .10 & 758 & 6.1 & 79 & 7.1 & 25400 & 22.9 \\
\hline $07 \ldots$ & 1600 & .30 & 758 & 4.7 & 64 & 6.7 & 31900 & 25.7 \\
\hline $10 \ldots$ & 1630 & .50 & 754 & 9.8 & 141 & 7.7 & 32500 & 27.6 \\
\hline $10 \ldots$ & 1634 & .10 & 754 & 9.8 & 136 & 8.3 & 23500 & 27.4 \\
\hline $21 \ldots$ & 1659 & .20 & 765 & 11.7 & 152 & 8.8 & 25900 & 24.3 \\
\hline $21 \ldots$ & 1700 & 1.1 & 765 & 12.8 & 170 & 8.3 & 32000 & 24.2 \\
\hline SEP & & & & & & & & \\
\hline $11 \ldots$ & 1630 & .30 & 768 & 11.2 & 144 & 8.7 & 28900 & 23.1 \\
\hline $11 \ldots$ & 1635 & 1.1 & 768 & 10.9 & 160 & 8.0 & 41300 & 27.8 \\
\hline $25 \ldots$ & 1628 & .20 & 761 & 9.2 & 112 & 8.0 & 34700 & 18.6 \\
\hline $25 \ldots$ & 1629 & .70 & 761 & 9.4 & 117 & 8.1 & 41500 & 18.1 \\
\hline $\mathrm{OCT}$ & & & & & & & & \\
\hline $11 \ldots$ & 1620 & .10 & 754 & 10.8 & 94 & 6.9 & 7300 & 7.9 \\
\hline $11 \ldots$ & 1622 & .90 & 754 & 4.7 & 57 & 7.1 & 38200 & 17.5 \\
\hline NOV & & & & & & & & \\
\hline $28 \ldots$ & 1540 & .00 & 758 & 11.6 & 87 & 5.8 & 90 & 3.0 \\
\hline $28 \ldots$ & 1545 & .60 & 758 & 11.0 & 82 & 5.8 & 90 & 3.0 \\
\hline
\end{tabular}


Table A3. Water-quality data collected May-November 2000 at station 102 (USGS identifier 442517068190501) Northeast Creek near Bar Harbor, ME - Continued.

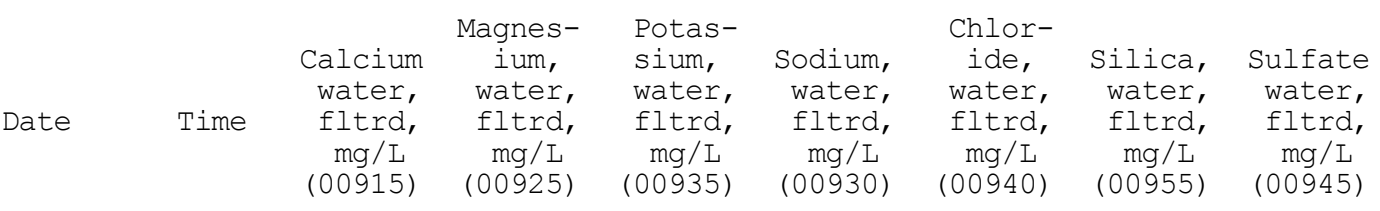

\begin{tabular}{|c|c|c|c|c|c|c|c|c|}
\hline $\begin{array}{c}\text { MAY } 2000 \\
17 \ldots \\
31 \ldots\end{array}$ & $\begin{array}{l}1850 \\
1355\end{array}$ & 3.44 & $\begin{array}{l}-- \\
4.04\end{array}$ & $1 .-\overline{30}$ & $\begin{array}{c}-- \\
43.3\end{array}$ & $\begin{array}{l}53.0 \\
65.0\end{array}$ & $\begin{array}{l}-- \\
.920\end{array}$ & $\begin{array}{l}6.0 \\
8.0\end{array}$ \\
\hline JUN & & & & & & & & \\
\hline $\begin{array}{l}13 \ldots \\
28 \ldots\end{array}$ & $\begin{array}{l}1725 \\
0855\end{array}$ & $\begin{array}{c}7.42 \\
12.6\end{array}$ & $\begin{array}{l}14.6 \\
31.0\end{array}$ & $\begin{array}{l}4.14 \\
8.44\end{array}$ & $\begin{array}{l}135 \\
313\end{array}$ & $\begin{array}{l}251 \\
494\end{array}$ & $\begin{array}{l}1.01 \\
.520\end{array}$ & $\begin{array}{l}31.0 \\
62.0\end{array}$ \\
\hline JUL & & & & & & & & \\
\hline $\begin{array}{l}10 \ldots \\
26 \ldots\end{array}$ & $\begin{array}{l}1710 \\
1615\end{array}$ & $\begin{array}{l}112 \\
27.0\end{array}$ & $\begin{array}{l}304 \\
69.9\end{array}$ & $\begin{array}{l}83.0 \\
20.0\end{array}$ & $\begin{array}{r}2690 \\
582\end{array}$ & $\begin{array}{r}5880 \\
114\end{array}$ & $\begin{array}{l}.920 \\
.340\end{array}$ & $\begin{array}{l}624 \\
145\end{array}$ \\
\hline AUG & & & & & & & & \\
\hline $\begin{array}{l}07 \ldots \\
21 \ldots\end{array}$ & $\begin{array}{l}1550 \\
1655\end{array}$ & $\begin{array}{l}172 \\
167\end{array}$ & $\begin{array}{l}486 \\
475\end{array}$ & $\begin{array}{l}149 \\
146\end{array}$ & $\begin{array}{l}4420 \\
4410\end{array}$ & $\begin{array}{r}11600 \\
7940\end{array}$ & $\begin{array}{l}.290 \\
.110\end{array}$ & $\begin{array}{l}1320 \\
1140\end{array}$ \\
\hline SEP & & & & & & & & \\
\hline $\begin{array}{l}11 \ldots \\
25 \ldots\end{array}$ & $\begin{array}{l}1625 \\
1625\end{array}$ & $\begin{array}{l}230 \\
260\end{array}$ & $\begin{array}{l}676 \\
809\end{array}$ & $\begin{array}{l}224 \\
280\end{array}$ & $\begin{array}{l}6180 \\
6120\end{array}$ & $\begin{array}{l}11400 \\
11900\end{array}$ & $\begin{array}{l}.160 \\
.090\end{array}$ & $\begin{array}{l}1790 \\
1590\end{array}$ \\
\hline OCT & & & & & & & & \\
\hline$\underset{N O V}{11 \ldots}$ & 1615 & 106 & 285 & 102 & 2170 & 4440 & 1.85 & 605 \\
\hline $28 \ldots$ & 1535 & 2.44 & 1.50 & 1.34 & 14.2 & 18.0 & 2.66 & 5.0 \\
\hline
\end{tabular}

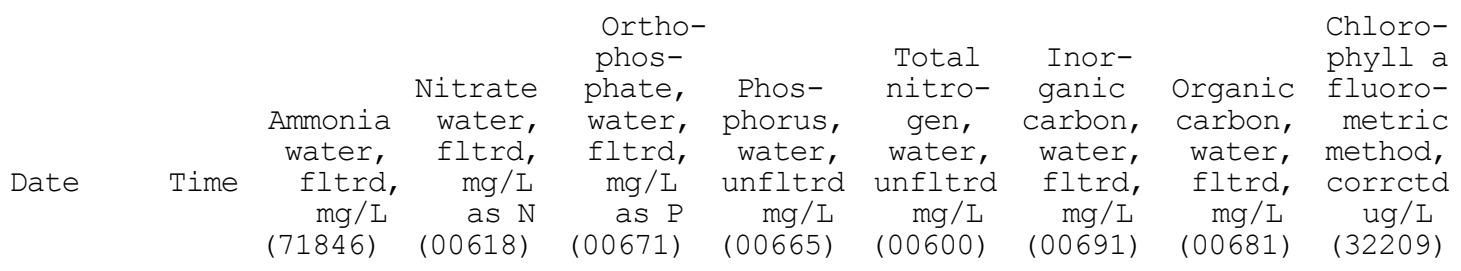

\begin{tabular}{|c|c|c|c|c|c|c|c|c|c|}
\hline \multicolumn{10}{|l|}{ MAY 2000} \\
\hline $17 \ldots$ & 1850 & -- & .00 & -- & 13.0 & .43 & -- & 13.5 & A1. 50 \\
\hline $31 \ldots$ & 1355 & .01 & .00 & 2.50 & 17.0 & .52 & 3.3 & 13.2 & $\mathrm{~A} 1.00$ \\
\hline \multicolumn{10}{|l|}{ JUN } \\
\hline $13 \ldots$ & $\begin{array}{l}1725 \\
855\end{array}$ & .02 & .01 & 6.60 & 25.0 & .55 & 4.1 & 14.3 & A3.40 \\
\hline \multicolumn{10}{|l|}{ JUL } \\
\hline $10 \ldots$ & 1710 & .02 & .00 & .000 & 22.0 & .62 & 8.9 & 7.3 & A4. 60 \\
\hline $26 \ldots$ & 1615 & .01 & .00 & 3.70 & 18.0 & .53 & -- & 9.6 & A1. 50 \\
\hline \multicolumn{10}{|l|}{ AUG } \\
\hline $07 \ldots$ & 1550 & .02 & .00 & 1.50 & 12.0 & .41 & 9.4 & 2.2 & A1. 80 \\
\hline $21 \ldots$ & 1655 & .00 & .00 & .000 & 9.00 & .49 & 6.6 & 2.6 & A1.20 \\
\hline \multicolumn{10}{|l|}{ SEP } \\
\hline $11 \ldots$ & 1625 & .06 & 18.1 & 3.30 & 17.0 & .77 & 10.8 & 2.8 & A3.80 \\
\hline $25 \ldots$ & 1625 & .17 & .00 & 4.60 & 36.0 & .54 & 15.0 & 2.1 & A5. 40 \\
\hline \multicolumn{10}{|l|}{ OCT } \\
\hline $11 \ldots$ & 1615 & .03 & .00 & 4.30 & 20.0 & .53 & 7.8 & 4.0 & A5. 50 \\
\hline \multicolumn{10}{|l|}{ NOV } \\
\hline $28 \ldots$ & 1535 & .02 & .00 & 4.50 & 21.0 & .51 & 1.9 & 14.7 & A. 600 \\
\hline
\end{tabular}


Table A4. Water-quality data collected May-November 2000 at station 103 (USGS identifier 442507068185301) Northeast Creek near Bar Harbor, ME [Numbers in parentheses are USGS NWIS parameter code numbers. Sampling depths are relative to the water surface. Abbreviations: A, average value; deg $\mathrm{C}$, degrees Celsius; fltrd, filtered; $\mathrm{mg} / \mathrm{L}$, milligrams per liter; $\mathrm{mm} \mathrm{Hg}$, millimeters of mercury; $\mathrm{N}$, nitrogen; P, phosphorus; std, standard; ug/L, micrograms per liter; unfltrd, unfiltered; uS/cm, microsiemens per centimeter; wat unf, unfiltered water; $<$, less than; --, missing data]. Chlorophyll-a results have been corrected for pheophytin and are reported as the mean of triplicate subsamples from a composited sample collected at each sampling date.

\begin{tabular}{|c|c|c|c|c|c|c|c|c|}
\hline Date & Time & $\begin{array}{c}\text { Sam- } \\
\text { pling } \\
\text { depth, } \\
\text { meters } \\
(00098)\end{array}$ & $\begin{array}{l}\text { Baro- } \\
\text { metric } \\
\text { pres- } \\
\text { sure, } \\
\text { mm Hg } \\
(00025)\end{array}$ & $\begin{array}{c}\text { Dis- } \\
\text { solved } \\
\text { oxygen, } \\
\text { mg/L } \\
(00300)\end{array}$ & $\begin{array}{c}\text { Dis- } \\
\text { solved } \\
\text { oxygen, } \\
\text { percent } \\
\text { of sat- } \\
\text { uration } \\
\text { (00301) }\end{array}$ & $\begin{array}{c}\text { pH, } \\
\text { water, } \\
\text { unfltrd } \\
\text { field, } \\
\text { std } \\
\text { units } \\
(00400)\end{array}$ & $\begin{array}{c}\text { Specif. } \\
\text { conduc- } \\
\text { tance, } \\
\text { wat unf } \\
\text { uS/ cm } \\
25 \text { degc } \\
(00095)\end{array}$ & $\begin{array}{c}\text { Temper- } \\
\text { ature, } \\
\text { water, } \\
\text { deg C } \\
(00010)\end{array}$ \\
\hline
\end{tabular}

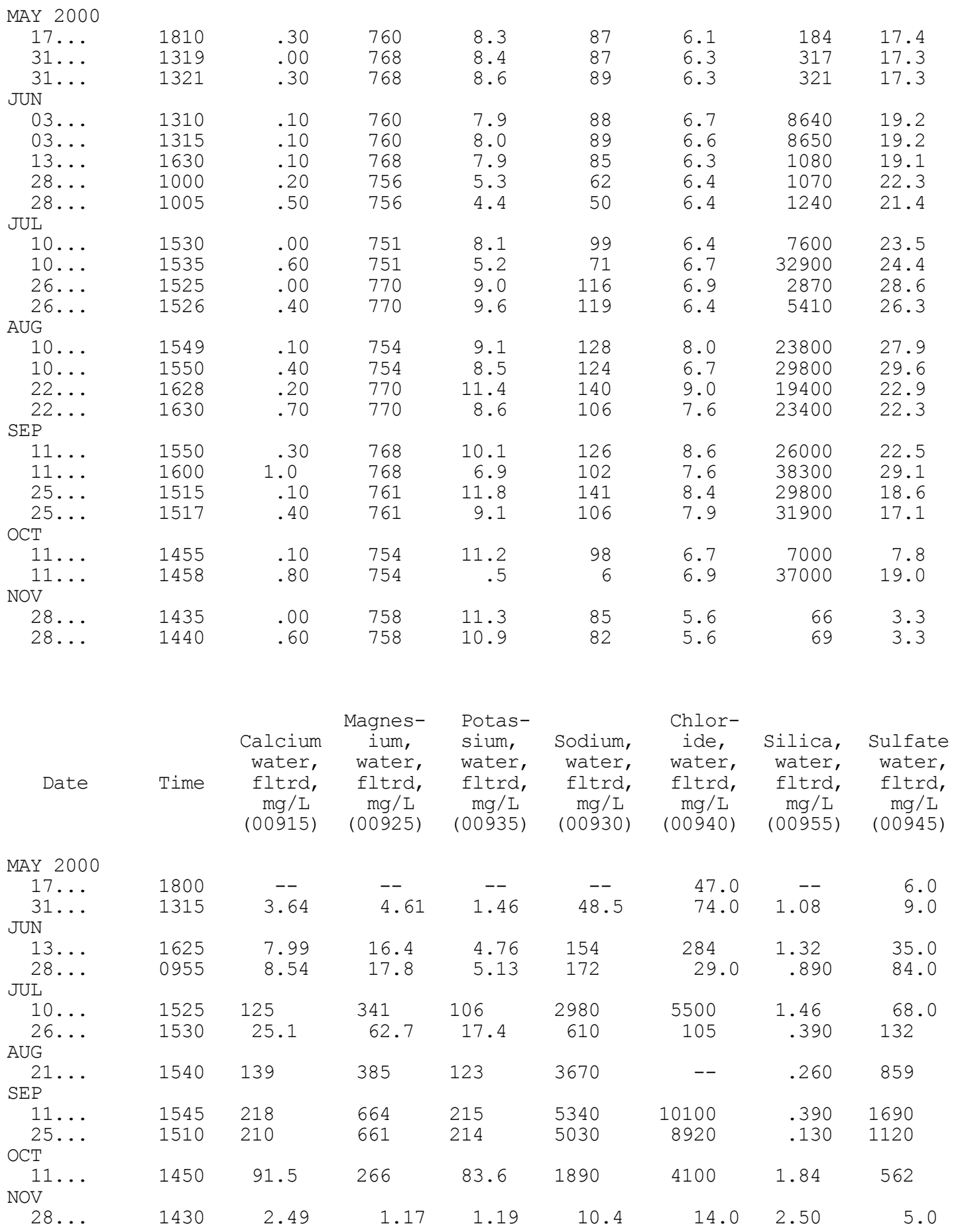


Table A4. Water-quality data collected May-November 2000 at station 103 (USGS identifier 442507068185301) Northeast Creek near Bar Harbor, ME - Continued.

\begin{tabular}{|c|c|c|c|c|c|c|c|c|}
\hline Time & $\begin{array}{c}\text { Ammonia } \\
\text { water, } \\
\text { fltrd, } \\
\text { mg/L }\end{array}$ & $\begin{array}{c}\text { Nitrate } \\
\text { water, } \\
\text { fltrd, } \\
\text { mg/L } \\
\text { as } N \\
(00618)\end{array}$ & $\begin{array}{c}\text { Ortho- } \\
\text { phos- } \\
\text { phate, } \\
\text { water, } \\
\text { fltrd, } \\
\text { mg/L } \\
\text { as P } \\
(00671)\end{array}$ & $\begin{array}{c}\text { Phos- } \\
\text { phorus, } \\
\text { water, } \\
\text { unfltrd } \\
\mathrm{mg} / \mathrm{L} \\
(00665)\end{array}$ & $\begin{array}{c}\text { Total } \\
\text { nitro- } \\
\text { gen, } \\
\text { water, } \\
\text { unfltrd } \\
\mathrm{mg} / \mathrm{L} \\
(00600)\end{array}$ & $\begin{array}{c}\text { Inor- } \\
\text { ganic } \\
\text { carbon, } \\
\text { water, } \\
\text { fltrd, } \\
\text { mg/L } \\
(00691)\end{array}$ & $\begin{array}{c}\text { Organic } \\
\text { carbon, } \\
\text { water, } \\
\text { fltrd, } \\
\mathrm{mg} / \mathrm{L} \\
(00681)\end{array}$ & $\begin{array}{l}\text { Chloro- } \\
\text { phyll a } \\
\text { fluoro- } \\
\text { metric } \\
\text { method, } \\
\text { corrctd } \\
\text { ug/L } \\
(32209)\end{array}$ \\
\hline
\end{tabular}

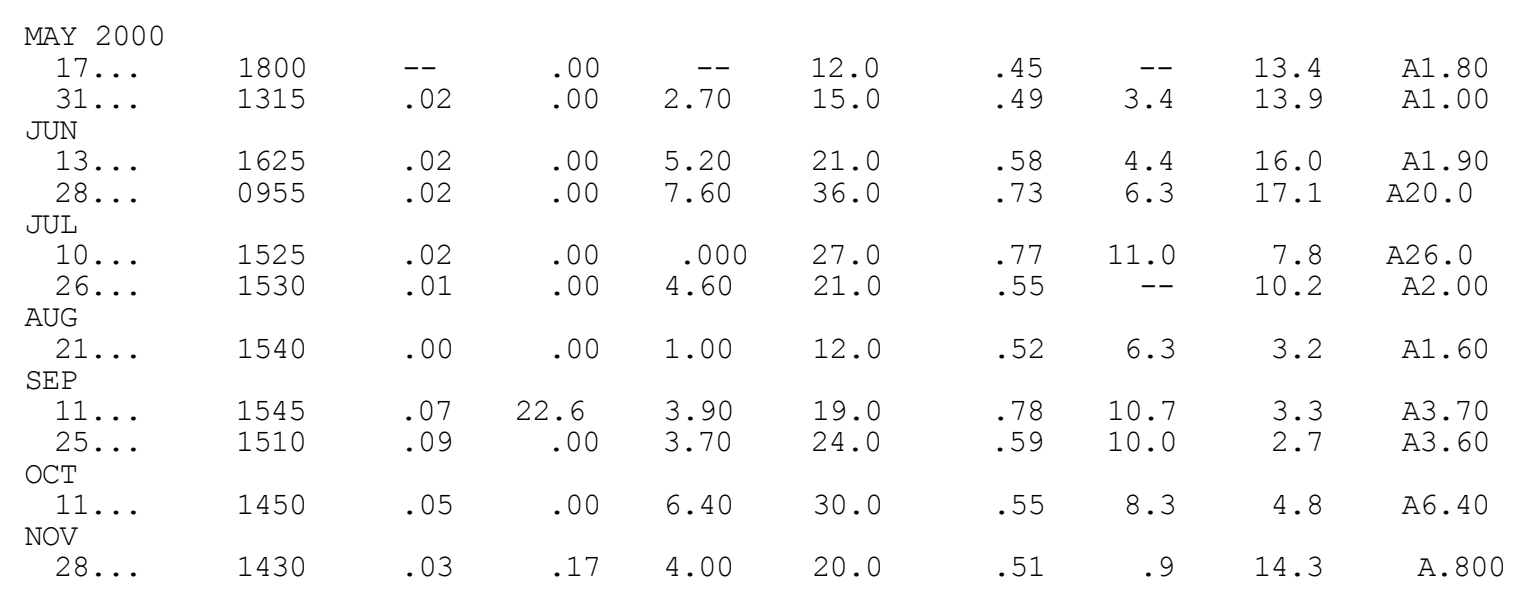


Hydrologic Data Summary for the Northeast Creek/Fresh Meadow Estuary, Acadia National Park, Maine, 2000-2001

Table A5. Water-quality data collected May-November 2000 at station 104 (USGS identifier 442509068181901) Northeast Creek near Bar Harbor, ME [Numbers in parentheses are USGS NWIS parameter code numbers. Sampling depths are relative to the water surface. Abbreviations: A, average value; deg C, degrees Celsius; fltrd, filtered; mg/L, milligrams per liter; mm $\mathrm{Hg}$, millimeters of mercury; $\mathrm{N}$, nitrogen; P, phosphorus; std, standard; ug/L, micrograms per liter; unfltrd, unfiltered; $\mathrm{uS} / \mathrm{cm}$, microsiemens per centimeter; wat unf, unfiltered water; <, less than; --, missing data]. Chlorophyll-a results have been corrected for pheophytin and are reported as the mean of triplicate subsamples from a composited sample collected at each sampling date.

\begin{tabular}{|c|c|c|c|c|c|c|c|c|}
\hline Date & Time & $\begin{array}{l}\text { Sam- } \\
\text { pling } \\
\text { depth, } \\
\text { meters } \\
(00098)\end{array}$ & $\begin{array}{l}\text { Baro- } \\
\text { metric } \\
\text { pres- } \\
\text { sure, } \\
\text { mm Hg } \\
(00025)\end{array}$ & $\begin{array}{c}\text { Dis- } \\
\text { solved } \\
\text { oxygen, } \\
\text { mg/L } \\
(00300)\end{array}$ & $\begin{array}{c}\text { Dis- } \\
\text { solved } \\
\text { oxygen, } \\
\text { percent } \\
\text { of sat- } \\
\text { uration } \\
\text { (00301) }\end{array}$ & $\begin{array}{c}\text { pH, } \\
\text { water, } \\
\text { unfltrd } \\
\text { field, } \\
\text { std } \\
\text { units } \\
(00400)\end{array}$ & $\begin{array}{l}\text { Specif. } \\
\text { conduc- } \\
\text { tance, } \\
\text { wat unf } \\
\text { uS/ cm } \\
25 \text { degc } \\
(00095)\end{array}$ & $\begin{array}{l}\text { Temper- } \\
\text { ature, } \\
\text { water, } \\
\text { deg C } \\
(00010)\end{array}$ \\
\hline
\end{tabular}

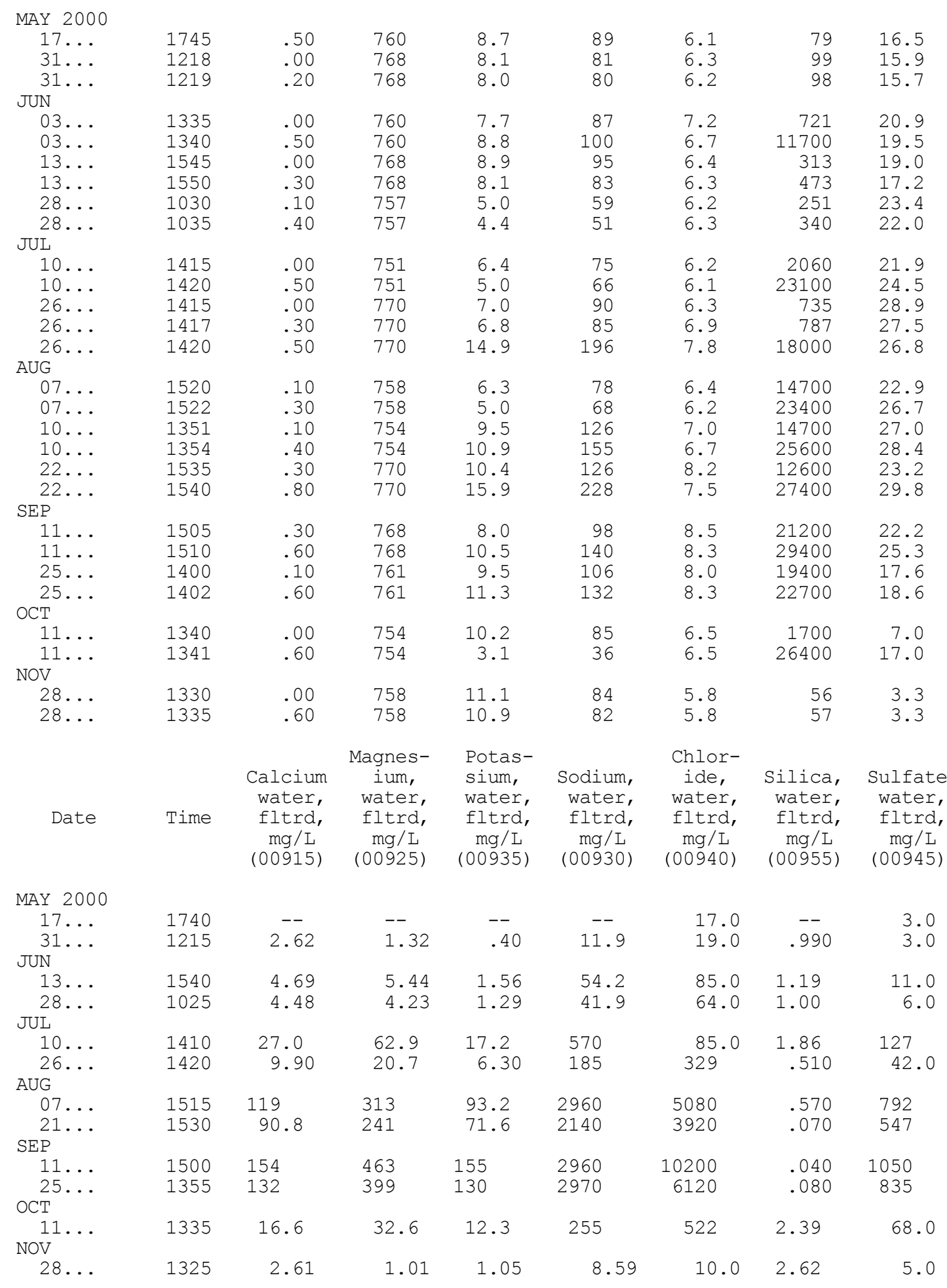


Table A5. Water-quality data collected May-November 2000 at station 104 (USGS identifier 442509068181901) Northeast Creek near Bar Harbor, ME - Continued.

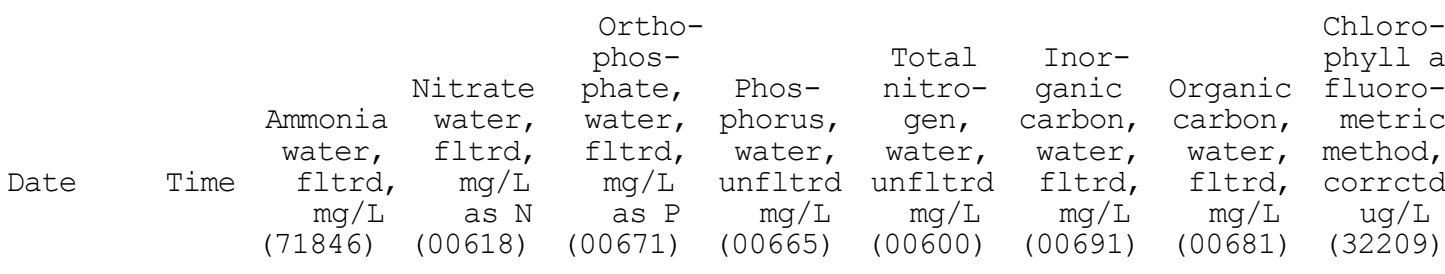

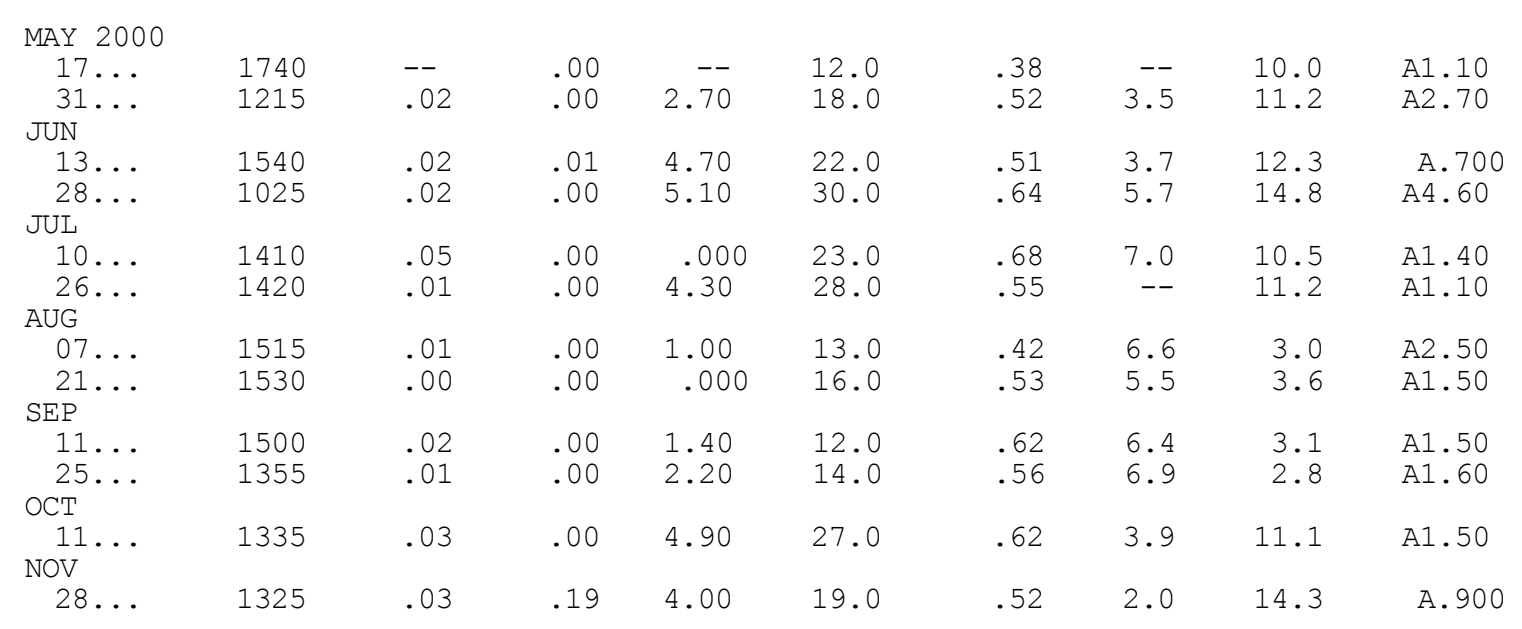


Hydrologic Data Summary for the Northeast Creek/Fresh Meadow Estuary, Acadia National Park, Maine, 2000-2001

Table A6. Water-quality data collected May-November 2000 at station 105 (USGS identifier 442516068175501) Northeast Creek near Bar Harbor, ME [Numbers in parentheses are USGS NWIS parameter code numbers. Sampling depths are relative to the water surface. Abbreviations: A, average value; deg C, degrees Celsius; fltrd, filtered; mg/L, milligrams per liter; mm $\mathrm{Hg}$, millimeters of mercury; $\mathrm{N}$, nitrogen; P, phosphorus; std, standard; ug/L, micrograms per liter; unfltrd, unfiltered; uS/cm, microsiemens per centimeter; wat unf, unfiltered water; <, less than; --, missing data]. Chlorophyll-a results have been corrected for pheophytin and are reported as the mean of triplicate subsamples from a composited sample collected at each sampling date.

\begin{tabular}{|c|c|c|c|c|c|c|c|c|}
\hline Date & Time & $\begin{array}{l}\text { Sam- } \\
\text { pling } \\
\text { depth, } \\
\text { meters } \\
(00098)\end{array}$ & $\begin{array}{l}\text { Baro- } \\
\text { metric } \\
\text { pres- } \\
\text { sure, } \\
\text { mm Hg } \\
(00025)\end{array}$ & $\begin{array}{c}\text { Dis- } \\
\text { solved } \\
\text { oxygen, } \\
\text { mg/L } \\
(00300)\end{array}$ & $\begin{array}{c}\text { Dis- } \\
\text { solved } \\
\text { oxygen, } \\
\text { percent } \\
\text { of sat- } \\
\text { uration } \\
\text { (00301) }\end{array}$ & $\begin{array}{c}\text { pH, } \\
\text { water, } \\
\text { unfltrd } \\
\text { field, } \\
\text { std } \\
\text { units } \\
(00400)\end{array}$ & $\begin{array}{c}\text { Specif. } \\
\text { conduc- } \\
\text { tance, } \\
\text { wat unf } \\
\text { uS/cm } \\
25 \text { degc } \\
(00095)\end{array}$ & $\begin{array}{c}\text { Temper- } \\
\text { ature, } \\
\text { water, } \\
\text { deg C } \\
(00010)\end{array}$ \\
\hline
\end{tabular}

\begin{tabular}{|c|c|c|c|c|c|c|c|c|}
\hline \multicolumn{9}{|c|}{ MAY 2000} \\
\hline $17 \ldots$ & 1700 & .50 & 760 & 8.1 & 83 & 5.9 & 59 & 16.6 \\
\hline $31 \ldots$ & 1133 & .20 & 768 & 7.5 & 75 & 6.2 & 65 & 15.6 \\
\hline $31 \ldots$ & 1136 & .30 & 768 & 7.5 & 74 & 6.1 & 65 & 15.5 \\
\hline $31 \ldots$ & 1137 & .50 & 768 & 7.6 & 74 & 6.1 & 69 & 15.0 \\
\hline \multicolumn{9}{|l|}{ JUN } \\
\hline $13 \ldots$ & 1430 & .20 & 768 & 7.1 & 74 & 6.2 & 122 & 17.7 \\
\hline $28 \ldots$ & 1050 & .10 & 756 & 3.1 & 36 & 6.0 & 112 & 21.9 \\
\hline $28 \ldots$ & 1055 & .50 & 756 & 1.3 & 14 & 5.8 & 133 & 19.2 \\
\hline \multicolumn{9}{|l|}{ JUL } \\
\hline $10 \ldots$ & 1300 & .00 & 751 & 5.0 & 57 & 6.0 & 6 & 20.8 \\
\hline $10 \ldots$ & 1305 & .50 & 751 & 3.4 & 40 & 5.9 & 10100 & 21.0 \\
\hline $26 \ldots$ & 1255 & .00 & 770 & 5.0 & 60 & 6.0 & 344 & 24.8 \\
\hline $26 \ldots$ & 1300 & .50 & 770 & 3.3 & 36 & 5.8 & 3360 & 20.5 \\
\hline \multicolumn{9}{|l|}{ AUG } \\
\hline $07 \ldots$ & 1410 & .00 & 758 & 5.4 & 63 & 6.0 & 4710 & 22.3 \\
\hline $07 \ldots$ & 1412 & .20 & 758 & 4.3 & 54 & 5.9 & 10100 & 24.3 \\
\hline $07 \ldots$ & 1415 & .40 & 758 & 2.1 & 30 & 6.0 & 27600 & 27.6 \\
\hline $10 \ldots$ & 1250 & .10 & 754 & 6.2 & 80 & 6.4 & 5000 & 27.3 \\
\hline $10 \ldots$ & 1255 & .20 & 754 & 5.6 & 74 & 6.3 & 5390 & 27.8 \\
\hline $10 \ldots$ & 1257 & .40 & 754 & 5.4 & 77 & 6.1 & 26500 & 28.7 \\
\hline $10 \ldots$ & 1258 & .40 & 754 & 5.6 & 79 & 6.2 & 23200 & 28.4 \\
\hline $22 \ldots$ & 1510 & .20 & 770 & 8.3 & 101 & 6.7 & 5300 & 25.0 \\
\hline $22 \ldots$ & 1512 & .60 & 770 & 6.8 & 85 & 6.3 & 12600 & 24.7 \\
\hline \multicolumn{9}{|l|}{$\operatorname{SEP}$} \\
\hline $11 \ldots$ & 1355 & .30 & 768 & 6.5 & 79 & 7.7 & 14900 & 22.7 \\
\hline $11 \ldots$ & 1400 & .90 & 768 & 8.7 & 129 & 8.2 & 28800 & 31.6 \\
\hline $25 \ldots$ & 1315 & .20 & 761 & 8.1 & 90 & 6.8 & 10700 & 18.3 \\
\hline $25 \ldots$ & 1318 & .50 & 761 & 12.6 & 160 & 7.5 & 17700 & 24.5 \\
\hline \multicolumn{9}{|l|}{$\mathrm{OCT}$} \\
\hline $11 \ldots$ & 1310 & .00 & 754 & 8.7 & 72 & 6.5 & 642 & 6.5 \\
\hline $11 \ldots$ & 1313 & .50 & 754 & 5.9 & 51 & 6.2 & 3650 & 7.7 \\
\hline \multicolumn{9}{|l|}{ NOV } \\
\hline \multirow{4}{*}{$\begin{array}{l}28 \ldots \\
28 \ldots\end{array}$} & 1300 & .00 & 758 & 11.0 & 83 & 5.8 & 61 & 3.3 \\
\hline & 1305 & .60 & 758 & 10.9 & 82 & 5.8 & 61 & 3.3 \\
\hline & & & Magnes- & Potas- & & Chlor- & & \\
\hline & & $\begin{array}{l}\text { Calcium } \\
\text { water, }\end{array}$ & $\begin{array}{c}\text { ium, } \\
\text { water, }\end{array}$ & & $\begin{array}{c}\text { Sodium, } \\
\text { water, }\end{array}$ & & $\begin{array}{l}\text { Silica, } \\
\text { water, }\end{array}$ & $\begin{array}{c}\text { Sulfate } \\
\text { water, }\end{array}$ \\
\hline \multirow[t]{3}{*}{ Date } & Time & fltrd, & fltrd, & fltrd, & fltrd, & fltrd, & fltrd, & fltrd, \\
\hline & & $\begin{array}{c}\mathrm{mg} / \mathrm{L} \\
(00915)\end{array}$ & $\begin{array}{c}\mathrm{mg} / \mathrm{L} \\
(00925)\end{array}$ & $\begin{array}{c}\mathrm{mg} / \mathrm{L} \\
(00935)\end{array}$ & $\begin{array}{c}\mathrm{mg} / \mathrm{L} \\
(00930)\end{array}$ & $\begin{array}{c}\mathrm{mg} / \mathrm{L} \\
(00940)\end{array}$ & $\begin{array}{c}\mathrm{mg} / \mathrm{L} \\
(00955)\end{array}$ & $\begin{array}{c}\mathrm{mg} / \mathrm{L} \\
(00945)\end{array}$ \\
\hline \multirow{2}{*}{\multicolumn{9}{|c|}{ MAY 2000}} \\
\hline & & & & & & & & \\
\hline $17 \ldots$ & 1650 & -- & -- & -- & -- & 12.0 & -- & 3.0 \\
\hline $31 \ldots$ & 1130 & 2.40 & .84 & .23 & 7.61 & 12.0 & .970 & 2.0 \\
\hline \multicolumn{9}{|l|}{ JUN } \\
\hline $13 \ldots$ & 1425 & 3.38 & 2.08 & .55 & 18.2 & 31.0 & 1.11 & 4.0 \\
\hline $28 \ldots$ & 1045 & 3.80 & 1.81 & .50 & 15.0 & 24.0 & 1.20 & 2.0 \\
\hline \multicolumn{9}{|l|}{ JUL } \\
\hline $10 \ldots$ & 1255 & 7.95 & 11.3 & 3.19 & 99.0 & 17.0 & 2.12 & 21.0 \\
\hline $26 \ldots$ & 1310 & 5.34 & 7.30 & 2.10 & 74.7 & 114 & 1.04 & 14.0 \\
\hline \multicolumn{9}{|l|}{ AUG } \\
\hline $07 \ldots$ & 1405 & 121 & 322 & 95.0 & 2950 & 5280 & .820 & 802 \\
\hline $21 \ldots$ & 1415 & 40.1 & 105 & 32.8 & 976 & 1630 & .850 & 232 \\
\hline \multicolumn{9}{|l|}{$\mathrm{SEP}$} \\
\hline $11 \ldots$ & 1350 & 109 & 327 & 106 & 2250 & 5600 & .070 & 653 \\
\hline $25 \ldots$ & 1310 & 74.3 & 214 & 68.3 & 1820 & -- & .230 & 461 \\
\hline \multicolumn{9}{|l|}{$\mathrm{OCT}$} \\
\hline $11 \ldots$ & 1300 & 10.1 & 11.1 & 6.42 & 91.6 & 181 & 2.78 & 29.0 \\
\hline $\begin{array}{l}\text { NOV } \\
28 \ldots\end{array}$ & 1255 & 2.99 & 1.07 & .92 & 8.73 & 11.0 & 2.69 & 5.0 \\
\hline
\end{tabular}


Table A6. Water-quality data collected May-November 2000 at station 105 (USGS identifier 442516068175501) Northeast Creek near Bar Harbor, ME - Continued.

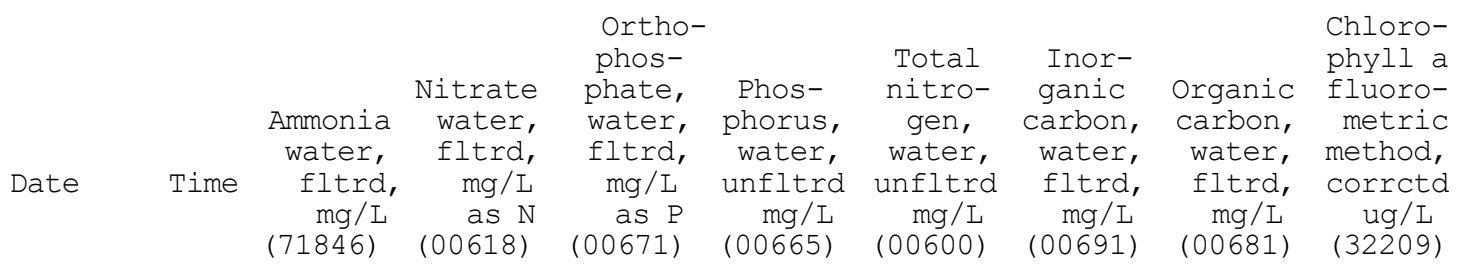

\begin{tabular}{|c|c|c|c|c|c|c|c|c|c|}
\hline \multicolumn{10}{|c|}{ MAY 2000} \\
\hline $17 \ldots$ & 1650 & -- & .01 & -- & 12.0 & .41 & -- & 9.3 & A. 700 \\
\hline $31 \ldots$ & 1130 & .01 & .00 & 3.30 & 26.0 & .52 & 3.6 & 10.0 & A4. 60 \\
\hline \multicolumn{10}{|l|}{ JUN } \\
\hline $\begin{array}{l}13 \ldots \\
28 \ldots\end{array}$ & $\begin{array}{l}1425 \\
1045\end{array}$ & $\begin{array}{l}.04 \\
.01\end{array}$ & $\begin{array}{l}.02 \\
.00\end{array}$ & $\begin{array}{c}8.60 \\
10.0\end{array}$ & $\begin{array}{l}34.0 \\
43.0\end{array}$ & $\begin{array}{l}.51 \\
.66\end{array}$ & $\begin{array}{l}4.1 \\
7.2\end{array}$ & $\begin{array}{l}11.7 \\
14.8\end{array}$ & $\begin{array}{l}\text { A7.30 } \\
\text { A3.10 }\end{array}$ \\
\hline \multicolumn{10}{|l|}{ JUL } \\
\hline $10 \ldots$ & 1255 & .03 & .00 & .000 & 29.0 & .72 & 6.5 & 10.4 & A. 800 \\
\hline $26 \ldots$ & 1310 & .01 & .00 & 4.80 & 27.0 & .53 & -- & 11.1 & A. 700 \\
\hline \multicolumn{10}{|l|}{ AUG } \\
\hline $07 \ldots$ & 1405 & .02 & .00 & 2.30 & 20.0 & .44 & 8.5 & 3.6 & A4. 50 \\
\hline $21 \ldots$ & 1415 & .00 & .00 & .000 & 19.0 & .55 & 4.5 & 6.2 & A1. 60 \\
\hline \multicolumn{10}{|l|}{ SEP } \\
\hline $11 \ldots$ & 1350 & .02 & .00 & 2.20 & 14.0 & .58 & 6.3 & 3.4 & A1. 30 \\
\hline $25 \ldots$ & 1310 & .01 & .00 & 2.50 & 19.0 & .50 & 5.9 & 3.6 & A1.20 \\
\hline \multicolumn{10}{|l|}{ OCT } \\
\hline $11 \ldots$ & 1300 & .02 & .00 & 5.80 & 31.0 & .67 & 4.5 & 11.4 & A1.10 \\
\hline NOV & & & & & & & & & \\
\hline 28 . & 1255 & .04 & .22 & 7.90 & 19.0 & .57 & 2.2 & 14.8 & A1.00 \\
\hline
\end{tabular}


Hydrologic Data Summary for the Northeast Creek/Fresh Meadow Estuary, Acadia National Park, Maine, 2000-2001

Table A7. Water-quality data collected May-November 2001 at station 100 (USGS identifier 442530068193901) Northeast Creek below Route 3 bridge near Bar Harbor, ME [Numbers in parentheses are USGS NWIS parameter code numbers. Sampling depths are relative to the water surface. Abbreviations: A, average value; deg C, degrees Celsius; fltrd, filtered; mg/L, milligrams per liter; $\mathrm{mm} \mathrm{Hg}$, millimeters of mercury; N, nitrogen; P, phosphorus; std, standard; ug/L, micrograms per liter; unfltrd, unfiltered; uS/cm, microsiemens per centimeter; wat unf, unfiltered water; $<$, less than; --, missing data]. Chlorophyll-a results have been corrected for pheophytin and are reported as the mean of triplicate subsamples from a composited sample collected at each sampling date.

\begin{tabular}{|c|c|c|c|c|c|c|c|c|}
\hline Date & Time & $\begin{array}{l}\text { Sam- } \\
\text { pling } \\
\text { depth, } \\
\text { meters } \\
(00098)\end{array}$ & $\begin{array}{l}\text { Baro- } \\
\text { metric } \\
\text { pres- } \\
\text { sure, } \\
\text { mm Hg } \\
(00025)\end{array}$ & $\begin{array}{c}\text { Dis- } \\
\text { solved } \\
\text { oxygen, } \\
\text { mg/L } \\
(00300)\end{array}$ & $\begin{array}{c}\text { Dis- } \\
\text { solved } \\
\text { oxygen, } \\
\text { percent } \\
\text { of sat- } \\
\text { uration } \\
\text { (00301) }\end{array}$ & $\begin{array}{c}\text { pH, } \\
\text { water, } \\
\text { unfltrd } \\
\text { field, } \\
\text { std } \\
\text { units } \\
(00400)\end{array}$ & $\begin{array}{l}\text { Specif. } \\
\text { conduc- } \\
\text { tance, } \\
\text { wat unf } \\
\text { uS/ cm } \\
25 \text { degC } \\
(00095)\end{array}$ & $\begin{array}{c}\text { Temper- } \\
\text { ature, } \\
\text { water, } \\
\text { deg C } \\
(00010)\end{array}$ \\
\hline
\end{tabular}

\begin{tabular}{|c|c|c|c|c|c|c|c|c|}
\hline \multicolumn{9}{|l|}{ MAY 2001} \\
\hline $22 \ldots$ & 1030 & .80 & 765 & 9.4 & 103 & 7.6 & 34400 & 13.8 \\
\hline $22 \ldots$ & 1040 & .80 & 765 & 9.2 & 101 & 7.7 & 36400 & 13.5 \\
\hline $22 \ldots$ & 1045 & .50 & 765 & 8.9 & 99 & 7.5 & 23300 & 16.4 \\
\hline \multicolumn{9}{|l|}{ JUN } \\
\hline $06 \ldots$ & 1155 & .30 & 756 & 7.1 & 80 & 6.3 & 4460 & 19.7 \\
\hline $06 \ldots$ & 1200 & .60 & 756 & 7.4 & 83 & 6.5 & 6830 & 19.3 \\
\hline $20 \ldots$ & 1145 & .40 & 762 & 8.1 & 100 & 7.0 & 23200 & 22.1 \\
\hline \multicolumn{9}{|l|}{ JUL } \\
\hline $03 \ldots$ & 0950 & .50 & 755 & 10.3 & 127 & 7.8 & 41300 & 17.1 \\
\hline $03 \ldots$ & 1000 & .80 & 755 & 9.7 & 117 & 7.8 & 47800 & \\
\hline $03 \ldots$ & 1010 & .60 & 755 & 10.5 & 128 & 7.8 & 45200 & 5.7 \\
\hline $18 \ldots$ & 1200 & .30 & 763 & 7.8 & 100 & 7.8 & 48400 & 18.4 \\
\hline \multicolumn{9}{|l|}{ AUG } \\
\hline $07 \ldots$ & 1405 & .10 & 745 & 12.4 & 190 & 8.0 & 50200 & 26.1 \\
\hline \multicolumn{9}{|l|}{ SEP } \\
\hline $\begin{array}{c}10 \ldots \\
\text { OCT }\end{array}$ & 1823 & .50 & 756 & 8.8 & 122 & 7.8 & 49400 & 7 \\
\hline $\begin{array}{l}01 \ldots \\
\text { NOV }\end{array}$ & 1125 & .50 & 759 & 7.1 & 82 & 7.6 & 50700 & 1 \\
\hline $06 \ldots$ & 1610 & .60 & 756 & 10.2 & 108 & 7.6 & 48300 & 8 \\
\hline
\end{tabular}

\begin{tabular}{|c|c|c|c|c|c|c|c|c|c|}
\hline Date & Time & $\begin{array}{c}\text { Silica, } \\
\text { water, } \\
\text { fltrd, } \\
\mathrm{mg} / \mathrm{L} \\
\text { (00955) }\end{array}$ & $\begin{array}{c}\text { Ammonia } \\
\text { water, } \\
\text { fltrd, } \\
\text { mg/L } \\
\text { (71846) }\end{array}$ & $\begin{array}{c}\text { Nitrate } \\
\text { water, } \\
\text { fltrd, } \\
\text { mg/L } \\
\text { as N } \\
(00618)\end{array}$ & $\begin{array}{c}\text { Nitrite } \\
+ \\
\text { nitrate } \\
\text { water } \\
\text { fltrd, } \\
\mathrm{mg} / \mathrm{L} \\
\text { as N } \\
(00631)\end{array}$ & $\begin{array}{c}\text { Ortho- } \\
\text { phos- } \\
\text { phate, } \\
\text { water, } \\
\text { fltrd, } \\
\text { mg/L } \\
\text { as P } \\
(00671)\end{array}$ & $\begin{array}{c}\text { Phos- } \\
\text { phorus, } \\
\text { water, } \\
\text { unfltrd } \\
\text { mg/L } \\
(00665)\end{array}$ & $\begin{array}{c}\text { Total } \\
\text { nitro- } \\
\text { gen, } \\
\text { water, } \\
\text { unfltrd } \\
\text { mg/L } \\
(00600)\end{array}$ & $\begin{array}{c}\text { Chloro- } \\
\text { phyll a } \\
\text { fluoro- } \\
\text { metric } \\
\text { method, } \\
\text { corrctd } \\
\text { ug/L } \\
\text { (32209) }\end{array}$ \\
\hline
\end{tabular}

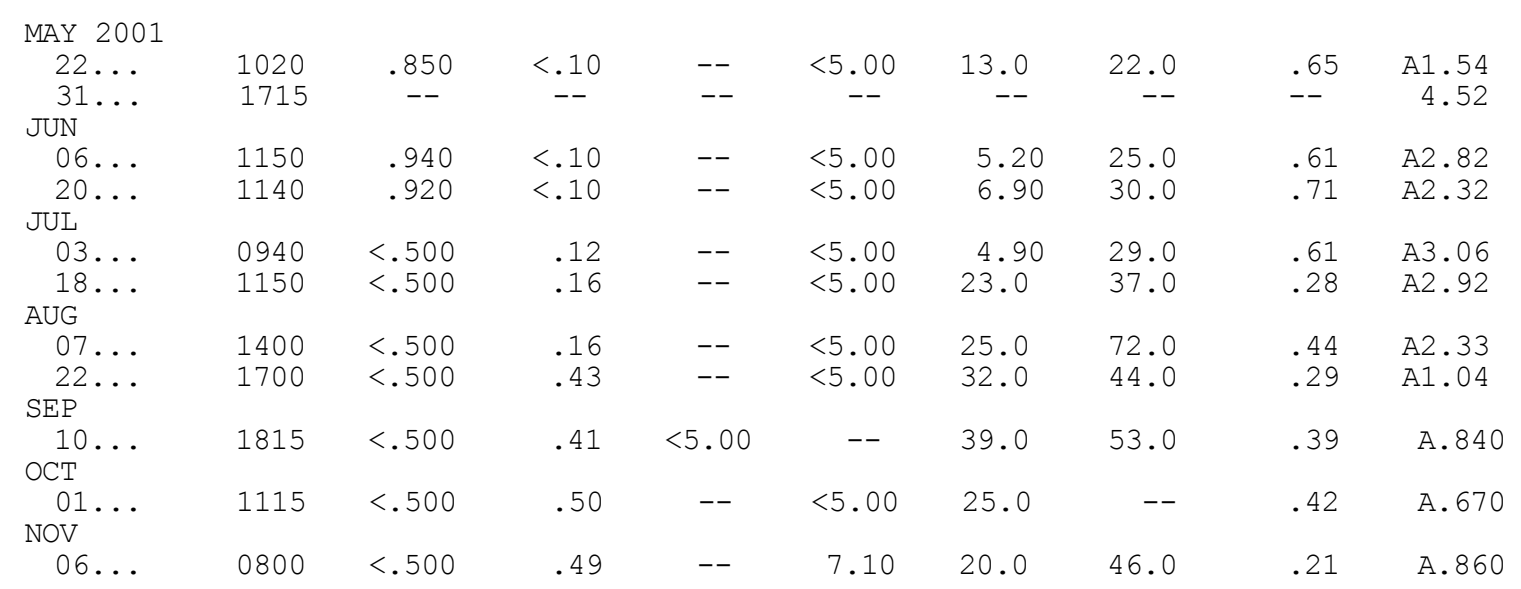


Table A8. Water-quality data collected May-November 2001 at station 101 (USGS identifier 01022820) Northeast Creek at Route 3 bridge near Bar Harbor, ME [Numbers in parentheses are USGS NWIS parameter code numbers. Sampling depths are relative to the water surface. Abbreviations: A, average value; deg C, degrees Celsius; fltrd, filtered; $\mathrm{mg} / \mathrm{L}$, milligrams per liter; $\mathrm{mm} \mathrm{Hg}$, millimeters of mercury; $\mathrm{N}$, nitrogen; P, phosphorus; std, standard; ug/L, micrograms per liter; unfltrd, unfiltered; uS/cm, microsiemens per centimeter; wat unf, unfiltered water; <, less than; --, missing data]. Chlorophyll-a results have been corrected for pheophytin and are reported as the mean of triplicate subsamples from a composited sample collected at each sampling date.

\begin{tabular}{|c|c|c|c|c|c|c|c|c|}
\hline Date & Time & $\begin{array}{l}\text { Sam- } \\
\text { pling } \\
\text { depth, } \\
\text { meters } \\
\text { (00098) }\end{array}$ & $\begin{array}{l}\text { Baro- } \\
\text { metric } \\
\text { pres- } \\
\text { sure, } \\
\text { mm Hg } \\
(00025)\end{array}$ & $\begin{array}{c}\text { Dis- } \\
\text { solved } \\
\text { oxygen, } \\
\text { mg/L } \\
(00300)\end{array}$ & $\begin{array}{c}\text { Dis- } \\
\text { solved } \\
\text { oxygen, } \\
\text { percent } \\
\text { of sat- } \\
\text { uration } \\
\text { (00301) }\end{array}$ & $\begin{array}{c}\text { pH, } \\
\text { water, } \\
\text { unfltrd } \\
\text { field, } \\
\text { std } \\
\text { units } \\
(00400)\end{array}$ & $\begin{array}{c}\text { Specif. } \\
\text { conduc- } \\
\text { tance, } \\
\text { wat unf } \\
\text { uS/cm } \\
25 \text { degc } \\
(00095)\end{array}$ & $\begin{array}{c}\text { Temper- } \\
\text { ature, } \\
\text { water, } \\
\text { deg C } \\
(00010)\end{array}$ \\
\hline
\end{tabular}

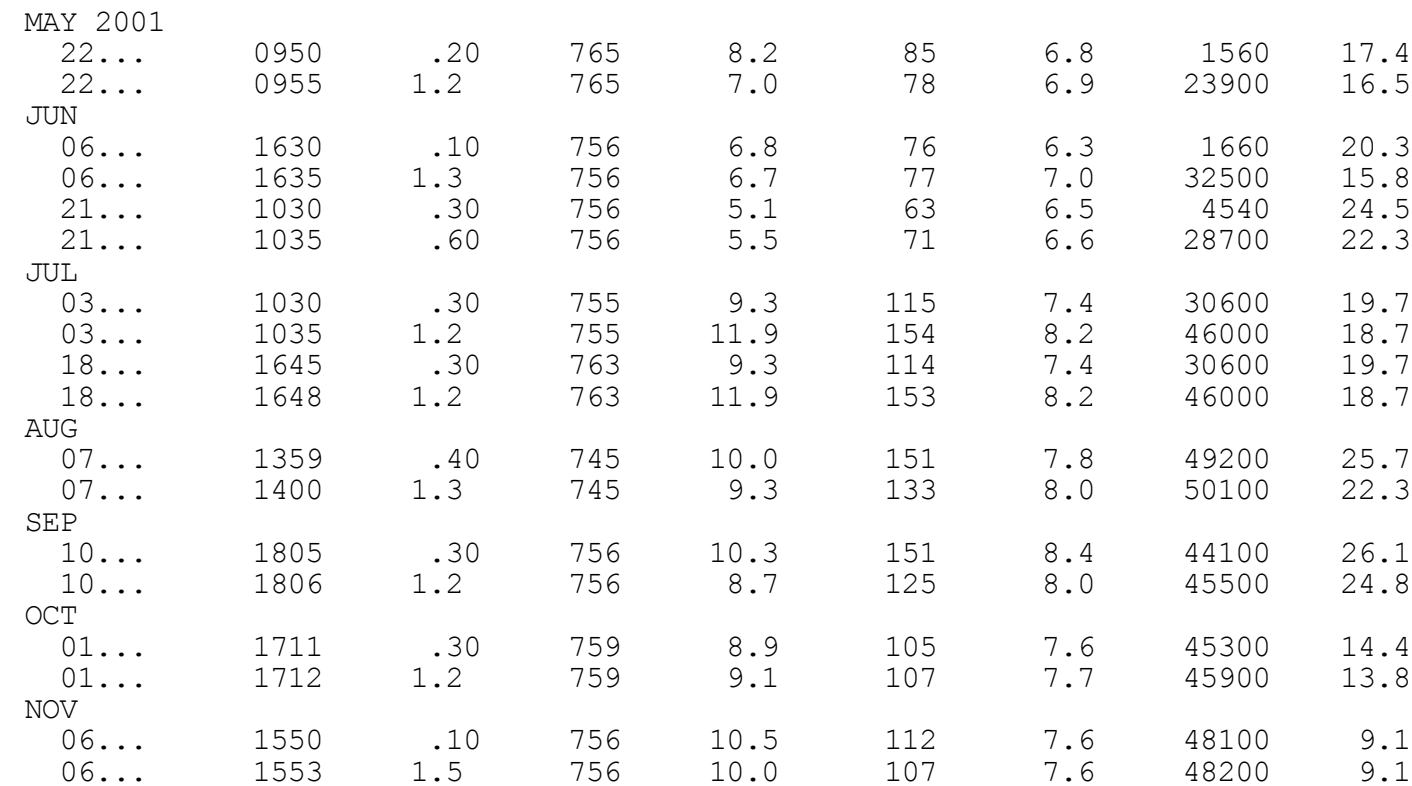

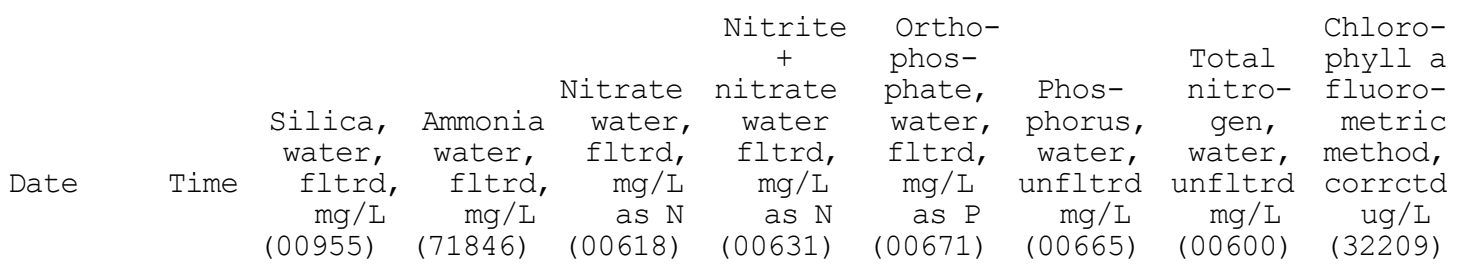

\begin{tabular}{|c|c|c|c|c|c|c|c|c|c|}
\hline \multicolumn{10}{|l|}{ HI 2001} \\
\hline $22 \ldots$ & 0940 & .840 & $<.10$ & -- & $<5.00$ & 17.0 & 26.0 & .68 & $\begin{array}{l}\text { A3. } 41 \\
\text { A1.38 }\end{array}$ \\
\hline $31 \ldots$ & 1700 & -- & -- & -- & -- & -- & -- & -- & 4.29 \\
\hline \multicolumn{10}{|l|}{ JUN } \\
\hline $06 \ldots$ & 1620 & 1.00 & $<.10$ & -- & $<5.00$ & 5.20 & 27.0 & .62 & A2. 15 \\
\hline $20 \ldots$ & 1715 & 1.20 & $<.10$ & -- & $<5.00$ & 7.10 & 31.0 & .79 & A4.03 \\
\hline \multicolumn{10}{|l|}{ JUL } \\
\hline $\begin{array}{l}02 \ldots \\
18 \ldots\end{array}$ & $\begin{array}{l}1630 \\
1640\end{array}$ & $\begin{array}{l}<.500 \\
<.500\end{array}$ & $\begin{array}{r}.18 \\
<.10\end{array}$ & $\begin{array}{l}-- \\
--\end{array}$ & $\begin{array}{l}<5.00 \\
70.0\end{array}$ & $\begin{array}{l}4.30 \\
6.70\end{array}$ & $\begin{array}{l}32.0 \\
31.0\end{array}$ & $\begin{array}{l}.25 \\
.36\end{array}$ & $\begin{array}{l}\text { A3. } 56 \\
\text { A } 4.75\end{array}$ \\
\hline \multicolumn{10}{|l|}{ AUG } \\
\hline $07 \ldots$ & 1350 & $<.500$ & .17 & -- & $<5.00$ & 3.60 & 27.0 & .36 & A1. 81 \\
\hline $22 \ldots$ & 1100 & $<.500$ & .40 & -- & $<5.00$ & 7.60 & 37.0 & .60 & A. 830 \\
\hline \multicolumn{10}{|l|}{ SEP } \\
\hline $\begin{array}{c}10 \ldots \\
\text { OCT }\end{array}$ & 1800 & $<.500$ & .38 & $<5.00$ & -- & 6.30 & 40.0 & .80 & A2. 85 \\
\hline $\begin{array}{c}01 \ldots \\
\text { NOV }\end{array}$ & 1700 & $<.500$ & .36 & -- & $<5.00$ & 7.40 & 28.0 & .68 & A2. 27 \\
\hline $06 \ldots$ & 1630 & $<.500$ & .41 & -- & 5.20 & 19.0 & 43.0 & .23 & A. 910 \\
\hline
\end{tabular}


Hydrologic Data Summary for the Northeast Creek/Fresh Meadow Estuary, Acadia National Park, Maine, 2000-2001

Table A9. Water-quality data collected May-November 2001 at station 102 (USGS identifier 442517068190501) Northeast Creek near Bar Harbor, ME [Numbers in parentheses are USGS NWIS parameter code numbers. Sampling depths are relative to the water surface. Abbreviations: A, average value; $\operatorname{deg} \mathrm{C}$, degrees Celsius; fltrd, filtered; $\mathrm{mg} / \mathrm{L}$, milligrams per liter; $\mathrm{mm} \mathrm{Hg}$, millimeters of mercury; $\mathrm{N}$, nitrogen; P, phosphorus; std, standard; ug/L, micrograms per liter; unfltrd, unfiltered; uS/cm, microsiemens per centimeter; wat unf, unfiltered water; <, less than; --, missing data]. Chlorophyll-a results have been corrected for pheophytin and are reported as the mean of triplicate subsamples from a composited sample collected at each sampling date.

\begin{tabular}{|c|c|c|c|c|c|c|c|c|}
\hline Date & Time & $\begin{array}{l}\text { Sam- } \\
\text { pling } \\
\text { depth, } \\
\text { meters } \\
(00098)\end{array}$ & $\begin{array}{l}\text { Baro- } \\
\text { metric } \\
\text { pres- } \\
\text { sure, } \\
\text { mm Hg } \\
(00025)\end{array}$ & $\begin{array}{c}\text { Dis- } \\
\text { solved } \\
\text { oxygen, } \\
\text { mg/L } \\
(00300)\end{array}$ & $\begin{array}{c}\text { Dis- } \\
\text { solved } \\
\text { oxygen, } \\
\text { percent } \\
\text { of sat- } \\
\text { uration } \\
\text { (00301) }\end{array}$ & $\begin{array}{c}\text { pH, } \\
\text { water, } \\
\text { unfltrd } \\
\text { field, } \\
\text { std } \\
\text { units } \\
(00400)\end{array}$ & $\begin{array}{c}\text { Specif. } \\
\text { conduc- } \\
\text { tance, } \\
\text { wat unf } \\
\text { uS / cm } \\
25 \mathrm{degC} \\
(00095)\end{array}$ & $\begin{array}{c}\text { Temper- } \\
\text { ature, } \\
\text { water, } \\
\text { deg C } \\
(00010)\end{array}$ \\
\hline
\end{tabular}

\begin{tabular}{|c|c|c|c|c|c|c|c|c|}
\hline $22 \ldots$ & 1100 & .20 & 765 & 7.3 & 79 & 6.8 & 844 & 19.2 \\
\hline $22 \ldots$ & 1105 & .70 & 765 & 7.6 & 77 & 6.7 & 1100 & 15.8 \\
\hline \multicolumn{9}{|l|}{ JUN } \\
\hline $06 \ldots$ & 1600 & .10 & 756 & 6.8 & 76 & 6.2 & 528 & 20.2 \\
\hline $06 \ldots$ & 1605 & .80 & 756 & 6.6 & 74 & 6.2 & 536 & 20.1 \\
\hline $20 \ldots$ & 1640 & .20 & 762 & 7.4 & 98 & 6.5 & 611 & 30.5 \\
\hline $20 \ldots$ & 1645 & .70 & 762 & 6.6 & 82 & 6.5 & 645 & 26.2 \\
\hline \multicolumn{9}{|l|}{ JUL } \\
\hline $03 \ldots$ & 1045 & .70 & 755 & 8.5 & 109 & 7.4 & 24300 & 22.8 \\
\hline $18 \ldots$ & 1606 & .70 & 763 & 6.7 & 93 & 7.7 & 32200 & 26.4 \\
\hline $18 \ldots$ & 1605 & .20 & 763 & 8.1 & 103 & 7.3 & 9140 & 25.9 \\
\hline \multicolumn{9}{|l|}{ AUG } \\
\hline $07 \ldots$ & 1334 & .30 & 745 & 9.8 & 147 & 8.2 & 32600 & 29.0 \\
\hline $07 \ldots$ & 1335 & .80 & 745 & 9.9 & 152 & 8.1 & 36900 & 29.3 \\
\hline \multicolumn{9}{|l|}{ SEP } \\
\hline $10 \ldots$ & 1700 & .20 & 756 & 10.3 & 147 & 8.2 & 37900 & 26.2 \\
\hline $10 \ldots$ & 1701 & .70 & 756 & 10.3 & 147 & 8.2 & 38200 & 25.9 \\
\hline \multicolumn{9}{|l|}{$\mathrm{OCT}$} \\
\hline $01 \ldots$ & 1557 & .20 & 759 & 9.8 & 126 & 7.6 & 42200 & 19.3 \\
\hline $01 \ldots$ & 1600 & .70 & 759 & 11.3 & 148 & 7.6 & 44300 & 20.4 \\
\hline \multicolumn{9}{|l|}{ NOV } \\
\hline $06 \ldots$ & 1522 & .10 & 756 & 12.6 & 133 & 7.9 & 41300 & 10.0 \\
\hline $06 \ldots$ & 1525 & .90 & 756 & 12.4 & 132 & 7.9 & 42000 & 10.2 \\
\hline
\end{tabular}

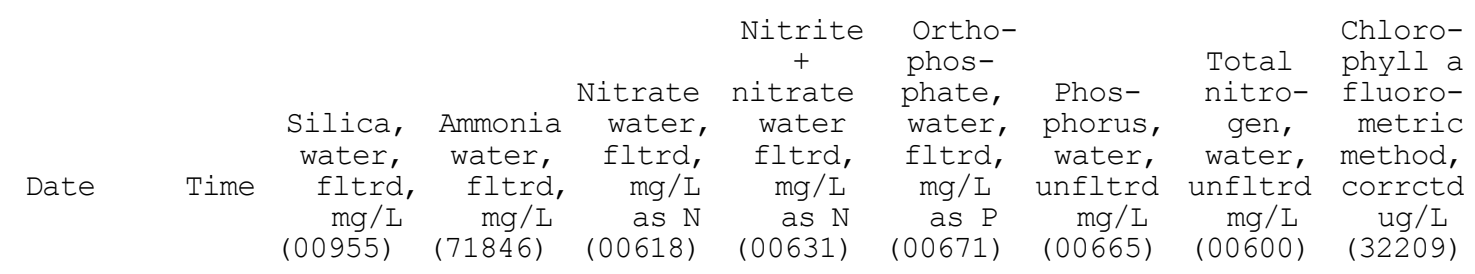

\begin{tabular}{|c|c|c|c|c|c|c|c|c|c|}
\hline \multicolumn{10}{|c|}{ MAY 2001} \\
\hline $\begin{array}{l}07 \ldots \\
22\end{array}$ & $\begin{array}{l}1510 \\
1050\end{array}$ & $1 . \overline{20}$ & $<-10$ & -- & $<5.00$ & $11^{--}$ & $22^{--}$ & -- & $\begin{array}{r}1.51 \\
A 1.79\end{array}$ \\
\hline 31 & 1630 & - & - & -- & - & ${ }_{-}^{+1}$ & 22.0 & .00 & 2.43 \\
\hline \multicolumn{10}{|l|}{ JUN } \\
\hline $06 \ldots$ & $\begin{array}{l}1555 \\
1630\end{array}$ & $\begin{array}{l}1.20 \\
1.20\end{array}$ & $\begin{array}{l}<.10 \\
<.10\end{array}$ & $\begin{array}{l}-- \\
--\end{array}$ & $\begin{array}{l}<5.00 \\
<5.00\end{array}$ & $\begin{array}{l}4.20 \\
5.90\end{array}$ & $\begin{array}{l}28.0 \\
34.0\end{array}$ & $\begin{array}{l}.66 \\
.74\end{array}$ & $\begin{array}{l}\text { A1.21 } \\
\text { A5.31 }\end{array}$ \\
\hline \multicolumn{10}{|l|}{ JUL } \\
\hline $02 \ldots$ & 1530 & $<.500$ & $<.10$ & -- & $<5.00$ & 6.00 & 20.0 & .52 & A2.85 \\
\hline $18 \ldots$ & 1600 & $<.500$ & $<.10$ & -- & $<5.00$ & 4.30 & 20.0 & .59 & А 3.19 \\
\hline \multicolumn{10}{|l|}{ AUG } \\
\hline $07 \ldots$ & 1330 & $<.500$ & $<.10$ & -- & $<5.00$ & 1.30 & 13.0 & .53 & A1.03 \\
\hline $22 \ldots$ & 1200 & $<.500$ & .26 & -- & $<5.00$ & 3.20 & 38.0 & .41 & A3.90 \\
\hline \multicolumn{10}{|l|}{ SEP } \\
\hline 10. & 1650 & $<.500$ & .17 & $<5.00$ & -- & 3.30 & 28.0 & .79 & A3.01 \\
\hline \multicolumn{10}{|l|}{ OCT } \\
\hline $01 \ldots$ & 1550 & $<.500$ & .18 & -- & $<5.00$ & $<1.00$ & 14.0 & .77 & A2.34 \\
\hline NOV $6 .$. & 1600 & $<.500$ & .22 & -- & $<5.00$ & 2.60 & 52.0 & .34 & A9.83 \\
\hline
\end{tabular}


Table A10. Water-quality data collected May-November 2001 at station 103 (USGS identifier 442507068185301) Northeast Creek near Bar Harbor, ME [Numbers in parentheses are USGS NWIS parameter code numbers. Sampling depths are relative to the water surface. Abbreviations: A, average value; deg C, degrees Celsius; fltrd, filtered; mg/L, milligrams per liter; $\mathrm{mm} \mathrm{Hg}$, millimeters of mercury; $\mathrm{N}$, nitrogen; P, phosphorus; std, standard; ug/L, micrograms per liter; unfltrd, unfiltered; uS/cm, microsiemens per centimeter; wat unf, unfiltered water; <, less than; --, missing data]. Chlorophyll-a results have been corrected for pheophytin and are reported as the mean of triplicate subsamples from a composited sample collected at each sampling date.

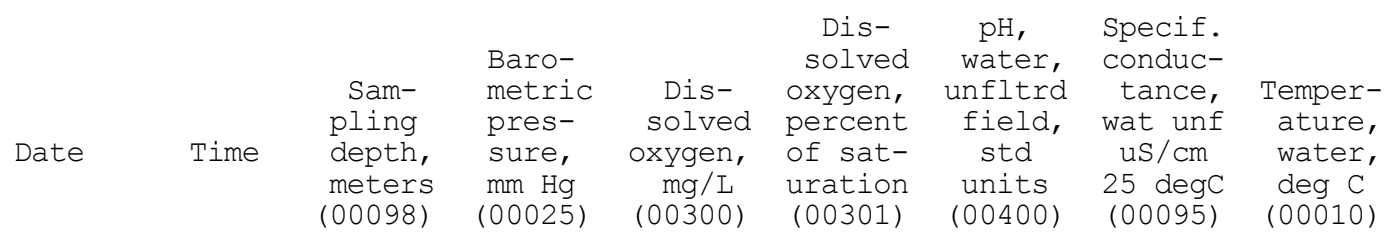

\begin{tabular}{|c|c|c|c|c|c|c|c|c|}
\hline \multicolumn{9}{|c|}{2001} \\
\hline $22 \ldots$ & 1205 & .20 & 765 & 8.0 & 85 & 6.8 & 452 & 18.4 \\
\hline $22 \ldots$ & 1207 & .70 & 765 & 7.6 & 81 & 6.7 & 2300 & 18.2 \\
\hline \multicolumn{9}{|l|}{ JUN } \\
\hline $06 \ldots$ & 1530 & .10 & 756 & 7.0 & 79 & 6.1 & 355 & 20.5 \\
\hline $06 \ldots$ & 1535 & .90 & 756 & 7.0 & 78 & 6.1 & 357 & 20.5 \\
\hline $20 \ldots$ & 1609 & .20 & 762 & 7.5 & 98 & 6.5 & 534 & 29.1 \\
\hline $20 \ldots$ & 1610 & .90 & 762 & 6.3 & 72 & 6.5 & 591 & 22.0 \\
\hline \multicolumn{9}{|l|}{ JUL } \\
\hline $03 \ldots$ & 1200 & .30 & 755 & 10.8 & 127 & 7.0 & 6050 & 21.8 \\
\hline $03 \ldots$ & 1201 & .60 & 755 & 8.9 & 110 & 6.9 & 13000 & 23.7 \\
\hline $18 \ldots$ & 1528 & .20 & 763 & 8.0 & 97 & 7.0 & 3340 & 25.0 \\
\hline $18 \ldots$ & 1530 & .80 & 763 & 2.1 & 28 & 6.6 & 24600 & 26.1 \\
\hline \multicolumn{9}{|l|}{ AUG } \\
\hline $07 \ldots$ & 1315 & 1.5 & 745 & 4.5 & 72 & 6.9 & 43200 & 30.3 \\
\hline \multicolumn{9}{|l|}{ SEP } \\
\hline $10 \ldots$ & 1610 & .80 & 756 & 8.3 & 116 & 7.9 & 35100 & 25.4 \\
\hline \multicolumn{9}{|l|}{$\mathrm{OCT}$} \\
\hline $01 \ldots$ & 1455 & .20 & 759 & 8.3 & 113 & 7.4 & 43800 & 22.2 \\
\hline $01 \ldots$ & 1457 & .80 & 759 & 6.9 & 97 & 7.4 & 47700 & 22.8 \\
\hline \multicolumn{9}{|l|}{ NOV } \\
\hline $06 \ldots$ & 1440 & .10 & 756 & 11.9 & 121 & 7.7 & 35800 & 9.3 \\
\hline $06 \ldots$ & 1442 & .60 & 756 & 11.8 & 120 & 7.7 & 35900 & \\
\hline
\end{tabular}

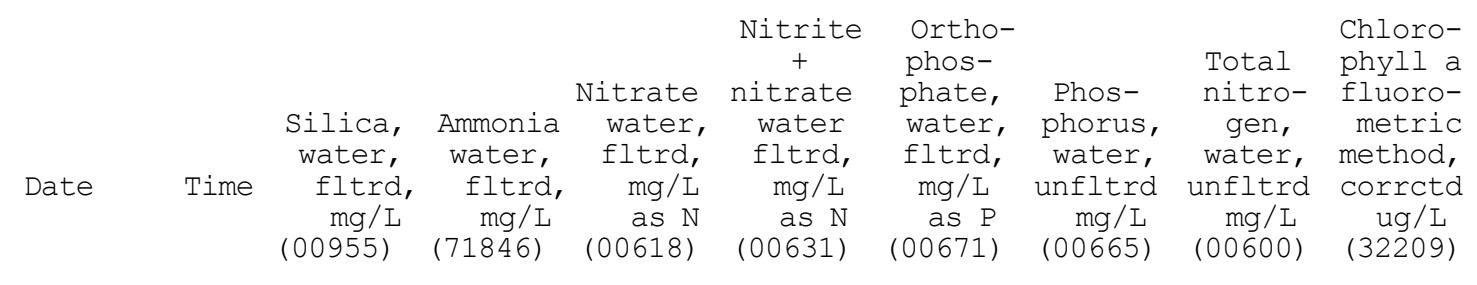

\begin{tabular}{|c|c|c|c|c|c|c|c|c|c|}
\hline \multicolumn{10}{|l|}{ MAY 2001} \\
\hline $07 \ldots$ & 1430 & -- & -- & -- & -- & -- & -- & -- & 4.06 \\
\hline $22 \ldots$ & 1200 & 1.10 & $<.10$ & -- & $<5.00$ & 11.0 & 21.0 & .66 & A1. 98 \\
\hline $31 \ldots$ & 1550 & -- & -- & -- & -- & -- & -- & -- & 3.13 \\
\hline \multicolumn{10}{|l|}{ JUN } \\
\hline $06 \ldots$ & 1520 & 1.20 & $<.10$ & -- & $<5.00$ & 4.50 & 26.0 & .75 & A1. 57 \\
\hline $20 \ldots$ & 1600 & 1.00 & $<.10$ & -- & $<5.00$ & 8.00 & 36.0 & .70 & A6.83 \\
\hline \multicolumn{10}{|l|}{ JUL } \\
\hline $02 \ldots$ & 1430 & $<.500$ & $<.10$ & -- & $<5.00$ & 5.50 & 25.0 & .56 & A4.19 \\
\hline $18 \ldots$ & 1520 & $<.500$ & $<.10$ & -- & $<5.00$ & 4.80 & 22.0 & .65 & A2. 88 \\
\hline \multicolumn{10}{|l|}{ AUG } \\
\hline $07 \ldots$ & 1310 & $<.500$ & $<.10$ & -- & $<5.00$ & 1.50 & 15.0 & .55 & A. 930 \\
\hline $22 \ldots$ & 1300 & $<.500$ & .24 & -- & $<5.00$ & 2.90 & 36.0 & .54 & A2. 74 \\
\hline \multicolumn{10}{|l|}{ SEP } \\
\hline $\begin{array}{c}10 \ldots \\
\text { OCT }\end{array}$ & 1600 & $<.500$ & .17 & $<5.00$ & -- & 2.70 & 18.0 & .78 & A2.09 \\
\hline$\underset{\text { NOV }}{01 \ldots}$ & 1450 & $<.500$ & .17 & -- & $<5.00$ & 1.40 & 12.0 & .66 & A2. 67 \\
\hline $06 \ldots$ & 1500 & $<.500$ & .23 & -- & $<5.00$ & 3.40 & 35.0 & .48 & A11.1 \\
\hline
\end{tabular}


Table A11. Water-quality data collected May-November 2001 at station 104 (USGS identifier 442509068181901) Northeast Creek near Bar Harbor, ME [Numbers in parentheses are USGS NWIS parameter code numbers. Sampling depths are relative to the water surface. Abbreviations: A, average value; $\operatorname{deg} \mathrm{C}$, degrees Celsius; fltrd, filtered; $\mathrm{mg} / \mathrm{L}$, milligrams per liter; $\mathrm{mm} \mathrm{Hg}$, millimeters of mercury; $\mathrm{N}$, nitrogen; P, phosphorus; std, standard; ug/L, micrograms per liter; unfltrd, unfiltered; uS/cm, microsiemens per centimeter; wat unf, unfiltered water; <, less than; --, missing data]. Chlorophyll-a results have been corrected for pheophytin and are reported as the mean of triplicate subsamples from a composited sample collected at each sampling date.

\begin{tabular}{|c|c|c|c|c|c|c|c|c|}
\hline ate & Time & $\begin{array}{c}\text { Sam- } \\
\text { pling } \\
\text { depth, } \\
\text { meters } \\
(00098)\end{array}$ & $\begin{array}{l}\text { Baro- } \\
\text { metric } \\
\text { pres- } \\
\text { sure, } \\
\text { mm Hg } \\
(00025)\end{array}$ & $\begin{array}{c}\text { Dis- } \\
\text { solved } \\
\text { oxygen, } \\
\text { mg/L } \\
(00300)\end{array}$ & $\begin{array}{c}\text { Dis- } \\
\text { solved } \\
\text { oxygen, } \\
\text { percent } \\
\text { of sat- } \\
\text { uration } \\
\text { (00301) }\end{array}$ & $\begin{array}{c}\text { pH, } \\
\text { water, } \\
\text { unfltrd } \\
\text { field, } \\
\text { std } \\
\text { units } \\
(00400)\end{array}$ & $\begin{array}{l}\text { Specif. } \\
\text { conduc- } \\
\text { tance, } \\
\text { wat unf } \\
\text { uS/ cm } \\
25 \text { degc } \\
(00095)\end{array}$ & $\begin{array}{c}\text { Temper- } \\
\text { ature, } \\
\text { water, } \\
\text { deg C } \\
(00010)\end{array}$ \\
\hline
\end{tabular}

\begin{tabular}{|c|c|c|c|c|c|c|c|c|}
\hline $22 \ldots$ & 1335 & .20 & 765 & 8.5 & 94 & 6.4 & 150 & 20.6 \\
\hline $22 \ldots$ & 1337 & .60 & 765 & 8.2 & 88 & 6.4 & 167 & 18.9 \\
\hline \multicolumn{9}{|l|}{ JUN } \\
\hline $06 \ldots$ & 1430 & .10 & 756 & 6.8 & 75 & 6.0 & 118 & 20.2 \\
\hline $06 \ldots$ & 1435 & .70 & 756 & 7.0 & 74 & 6.2 & 118 & 17.6 \\
\hline $20 \ldots$ & 1450 & .20 & 762 & 7.8 & 99 & 6.5 & 162 & 27.0 \\
\hline $20 \ldots$ & 1452 & .60 & 762 & 9.1 & 107 & 6.9 & 178 & 23.6 \\
\hline \multicolumn{9}{|l|}{ JUL } \\
\hline $03 \ldots$ & 1415 & .30 & 755 & 12.0 & 139 & 7.3 & 859 & 22.1 \\
\hline $03 \ldots$ & 1416 & .60 & 755 & 11.2 & 127 & 7.3 & 1110 & 20.8 \\
\hline $18 \ldots$ & 1441 & .20 & 763 & 8.2 & 96 & 6.9 & 284 & 23.5 \\
\hline $18 \ldots$ & 1443 & .60 & 763 & 4.1 & 45 & 6.4 & 447 & 20.0 \\
\hline \multicolumn{9}{|l|}{ AUG } \\
\hline $07 \ldots$ & 1234 & .30 & 745 & 9.2 & 128 & 8.1 & 15100 & 28.9 \\
\hline \multicolumn{9}{|l|}{ SEP } \\
\hline $10 \ldots$ & 1513 & .50 & 756 & 9.1 & 125 & 8.0 & 32400 & 25.3 \\
\hline \multicolumn{9}{|l|}{ OCT } \\
\hline $01 \ldots$ & $\begin{array}{l}1049 \\
1352\end{array}$ & .20 & 759 & 7.0 & 101 & 6 & $\begin{array}{l}32100 \\
01300\end{array}$ & 16.4 \\
\hline \multicolumn{9}{|l|}{ NOV } \\
\hline $06 \ldots$ & 1323 & .10 & 756 & 10.9 & 110 & 7.2 & 31200 & 9.9 \\
\hline $06 \ldots$ & 1330 & .80 & 756 & 12.1 & 125 & 7.4 & 31300 & 11.0 \\
\hline
\end{tabular}

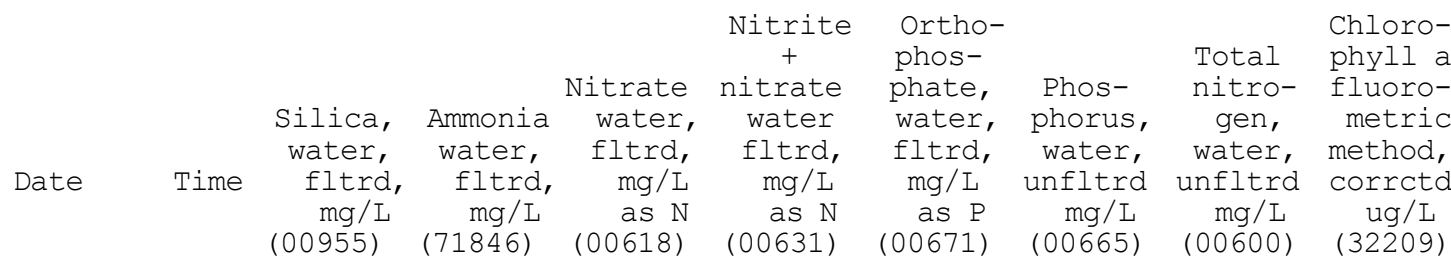

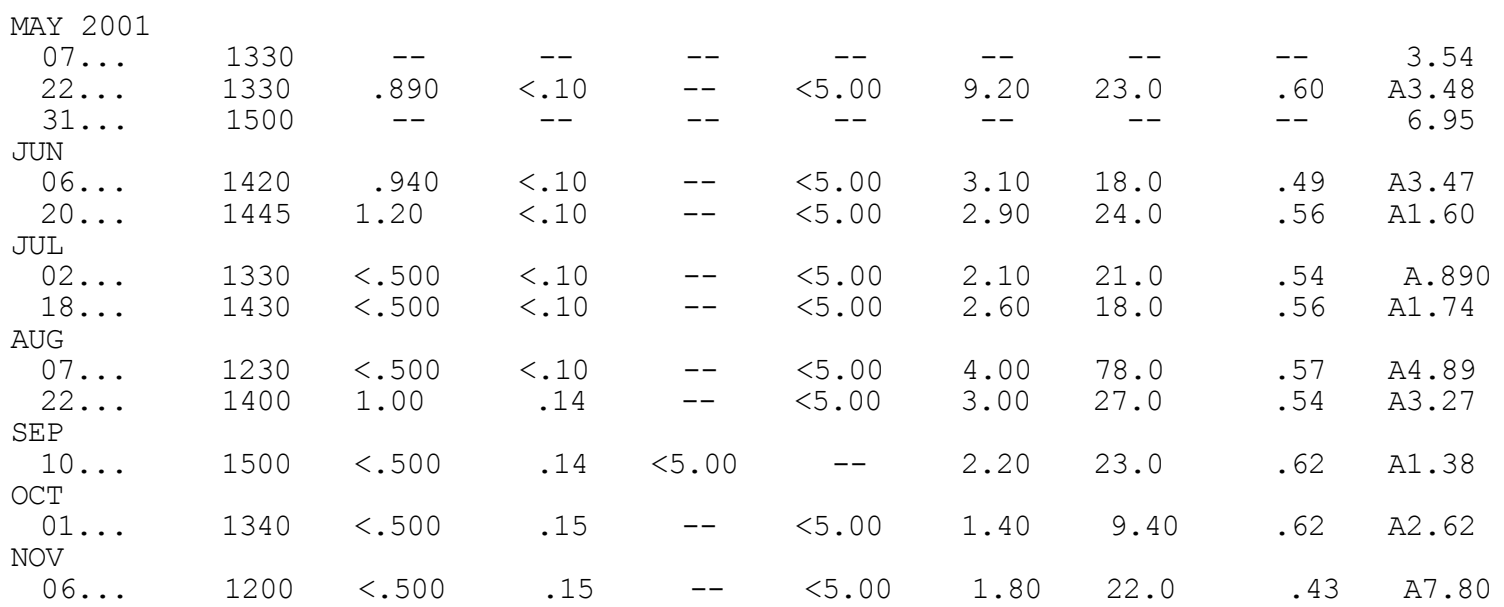


Table A12. Water-quality data collected May-November 2001 at station 105 (USGS identifier 442516068175501) Northeast Creek near Bar Harbor, ME [Numbers in parentheses are USGS NWIS parameter code numbers. Sampling depths are relative to the water surface. Abbreviations: A, average value; deg C, degrees Celsius; fltrd, filtered; mg/L, milligrams per liter; mm $\mathrm{Hg}$, millimeters of mercury; N, nitrogen; P, phosphorus; std, standard; ug/L, micrograms per liter; unfltrd, unfiltered; uS/cm, microsiemens per centimeter; wat unf, unfiltered water; <, less than; --, missing data]. Chlorophyll-a results have been corrected for pheophytin and are reported as the mean of triplicate subsamples from a composited sample collected at each sampling date.

\begin{tabular}{|c|c|c|c|c|c|c|c|c|}
\hline Date & Time & $\begin{array}{l}\text { Sam- } \\
\text { pling } \\
\text { depth, } \\
\text { meters } \\
(00098)\end{array}$ & $\begin{array}{l}\text { Baro- } \\
\text { metric } \\
\text { pres- } \\
\text { sure, } \\
\text { mm Hg } \\
(00025)\end{array}$ & $\begin{array}{c}\text { Dis- } \\
\text { solved } \\
\text { oxygen, } \\
\text { mg/L } \\
(00300)\end{array}$ & $\begin{array}{l}\text { Dis- } \\
\text { solved } \\
\text { oxygen, } \\
\text { percent } \\
\text { of sat- } \\
\text { uration } \\
\text { (00301) }\end{array}$ & $\begin{array}{l}\mathrm{pH}, \\
\text { water, } \\
\text { unfltrd } \\
\text { field, } \\
\text { std } \\
\text { units } \\
(00400)\end{array}$ & $\begin{array}{l}\text { Specif. } \\
\text { conduc- } \\
\text { tance, } \\
\text { wat unf } \\
\text { uS/cm } \\
25 \text { degC } \\
(00095)\end{array}$ & $\begin{array}{c}\text { Temper- } \\
\text { ature, } \\
\text { water, } \\
\text { deg C } \\
(00010)\end{array}$ \\
\hline
\end{tabular}

\begin{tabular}{|c|c|c|c|c|c|c|c|c|}
\hline \multicolumn{9}{|l|}{ Y 200} \\
\hline $22 \ldots$ & 1507 & .70 & 765 & 8.0 & 79 & $\begin{array}{l}6.3 \\
6.2\end{array}$ & $\begin{array}{l}115 \\
102\end{array}$ & $\begin{array}{l}19.1 \\
15.1\end{array}$ \\
\hline \multicolumn{9}{|l|}{ JUN } \\
\hline $06 \ldots$ & 1345 & .20 & 756 & 7.0 & 77 & 6.0 & 116 & 19.5 \\
\hline $06 \ldots$ & 1350 & .80 & 756 & 6.7 & 71 & 6.0 & 102 & 17.9 \\
\hline $20 \ldots$ & 1350 & .20 & 762 & 7.1 & 90 & 6.2 & 97 & 27.2 \\
\hline $20 \ldots$ & 1352 & .70 & 762 & 7.8 & 90 & 6.2 & 96 & 22.4 \\
\hline \multicolumn{9}{|l|}{ JUL } \\
\hline $03 \ldots$ & 1430 & .30 & 755 & 7.8 & 92 & 6.8 & 140 & 23.1 \\
\hline $03 \ldots$ & 1432 & .80 & 755 & 7.9 & 86 & 6.9 & 121 & 19.1 \\
\hline $18 \ldots$ & 1330 & .20 & 763 & 7.4 & 88 & 6.3 & 85 & 23.9 \\
\hline $18 \ldots$ & 1332 & .70 & 763 & 4.9 & 53 & 6.2 & 102 & 19.2 \\
\hline \multicolumn{9}{|l|}{ AUG } \\
\hline $07 \ldots$ & 1159 & .50 & 745 & 10.0 & 152 & 5.8 & 22800 & 32.3 \\
\hline \multicolumn{9}{|l|}{ SEP } \\
\hline $10 \ldots$ & 1343 & .20 & 756 & 7.5 & 103 & 7.0 & 29400 & 25.8 \\
\hline $10 \ldots$ & 1345 & .70 & 756 & 8.5 & 121 & 6.9 & 31100 & 27.3 \\
\hline \multicolumn{9}{|l|}{ OCT } \\
\hline $01 \cdots$ & 1247 & .70 & $\begin{array}{l}159 \\
759\end{array}$ & 12.9 & 171 & 7.5 & 39900 & 22.0 \\
\hline \multicolumn{9}{|l|}{ NOV } \\
\hline $06 \ldots$ & 1155 & .10 & 756 & 10.2 & 102 & 6.8 & 29600 & 9.9 \\
\hline $06 \ldots$ & 1200 & .50 & 756 & 10.9 & 114 & 7.3 & 31400 & 11.7 \\
\hline
\end{tabular}

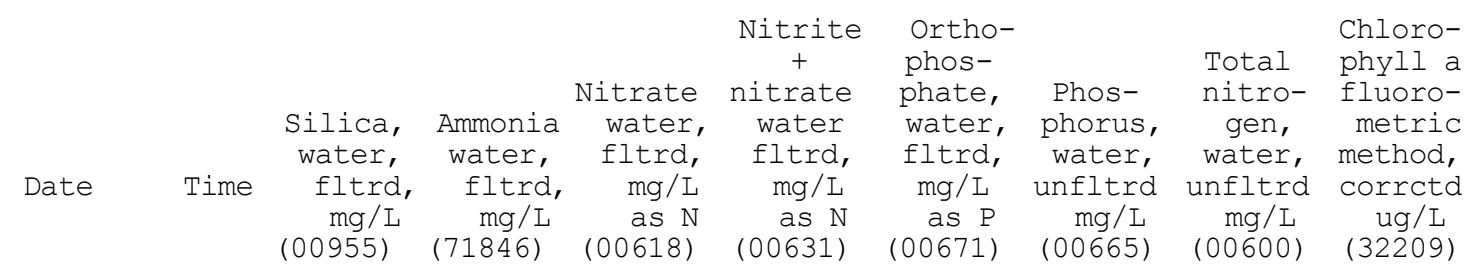

\begin{tabular}{|c|c|c|c|c|c|c|c|c|c|}
\hline $07 \ldots$ & 1230 & -- & -- & -- & -- & -- & -- & -- & 2.19 \\
\hline $22 \ldots$ & 1500 & .910 & $<.10$ & -- & $<5.00$ & 8.50 & 21.0 & .54 & A1. 56 \\
\hline $31 \ldots$ & 1420 & -- & -- & -- & -- & -- & -- & -- & 3.63 \\
\hline \multicolumn{10}{|l|}{ JUN } \\
\hline $06 \ldots$ & 1340 & 1.00 & $<.10$ & -- & $<5.00$ & 2.90 & 21.0 & .46 & A1. 81 \\
\hline $20 \ldots$ & 1340 & 1.70 & $<.10$ & -- & $<5.00$ & -- & 24.0 & .51 & A1.00 \\
\hline \multicolumn{10}{|l|}{ JUL } \\
\hline $02 \ldots$ & 1230 & 1.20 & $<.10$ & -- & $<5.00$ & 2.50 & 27.0 & .53 & A1.95 \\
\hline $18 \ldots$ & 1320 & .800 & $<.10$ & -- & $<5.00$ & 6.20 & 28.0 & .60 & A1.99 \\
\hline \multicolumn{10}{|l|}{ AUG } \\
\hline $07 \ldots$ & 1150 & $<.500$ & $<.10$ & -- & $<5.00$ & 2.80 & 18.0 & .56 & A4.71 \\
\hline$\underset{S F P}{22} \cdots$ & 1500 & .720 & .13 & -- & $<5.00$ & 5.50 & 36.0 & .55 & A10. 8 \\
\hline \multicolumn{10}{|l|}{ SEP } \\
\hline OCT & 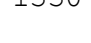 & .300 & 2.10 & $<0.00$ & & 1.90 & 21.0 & .01 & $A \perp .13$ \\
\hline $01 \ldots$ & 1240 & $<.500$ & .15 & -- & $<5.00$ & 1.40 & 11.0 & .69 & A3. 60 \\
\hline 06. & 1100 & $<.500$ & $<.10$ & -- & 5.10 & 1.60 & 21.0 & .45 & A5.90 \\
\hline
\end{tabular}


Table A13. --Water-quality assurance records, replicate samples collected May-November 2000, Northeast Creek stations 100 through 105. Replicate samples are in bold. [Numbers in parentheses are USGS NWIS parameter code numbers. Abbreviations: fltrd, filtered; mg/L, milligrams per liter; N, nitrogen; P, phosphorus; std, standard; ug/L, micrograms per liter; unfltrd, unfiltered; uS/cm, microsiemens per centimeter; wat unf, unfiltered water; $<$, less than; --, missing data].

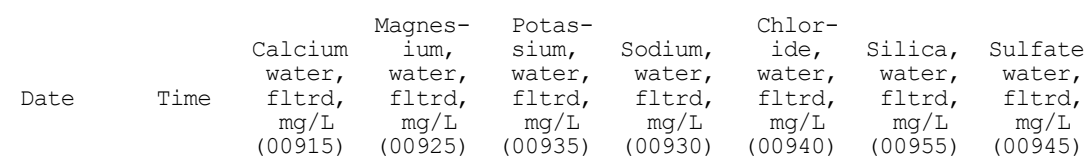

442530068193901 Northeast Creek monitoring station 100

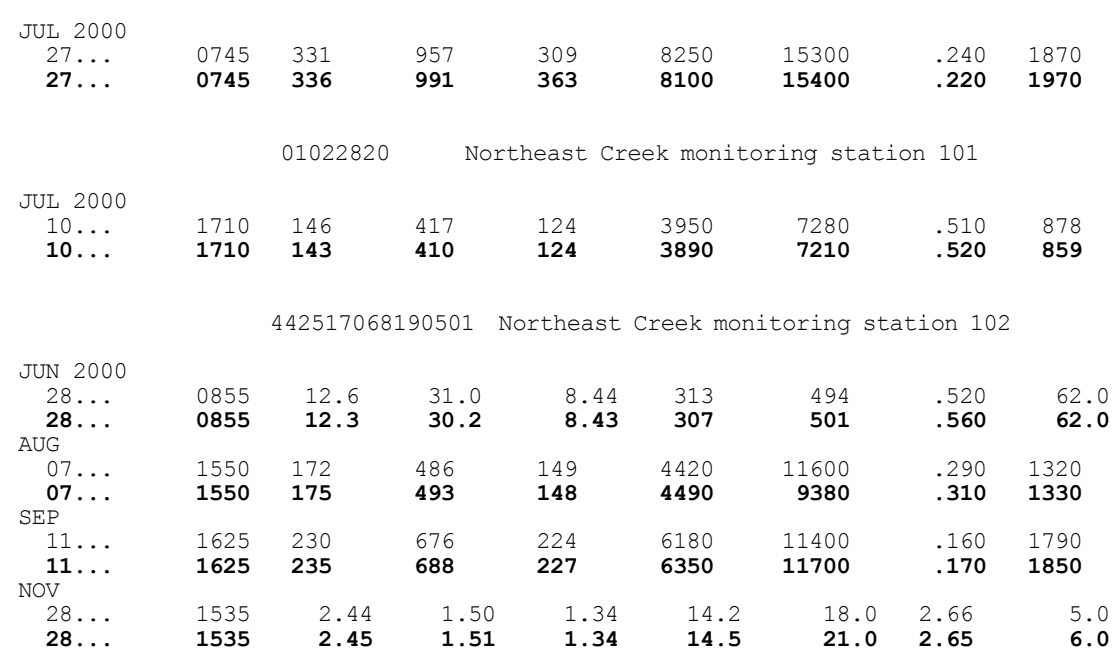

442507068185301 Northeast Creek monitoring station 103

$\begin{array}{crrrrrrrr}\text { JUL } 2000 & & & & & & & \\ 10 \ldots & 1525 & 125 & 341 & 106 & 2980 & 5500 & 1.46 & 68.0 \\ \mathbf{1 0} \ldots & \mathbf{1 5 2 5} & \mathbf{1 2 5} & \mathbf{3 4 1} & \mathbf{1 0 6} & \mathbf{2 9 8 0} & \mathbf{5 5 3 0} & \mathbf{1 . 4 4} & \mathbf{6 9 . 0} \\ 26 \ldots & 1530 & 25.1 & 62.7 & 17.4 & 610 & 105 & .390 & 132 \\ \mathbf{2 6} \ldots & \mathbf{1 5 3 0} & \mathbf{2 4 . 9} & \mathbf{6 2 . 5} & \mathbf{1 7 . 1} & \mathbf{6 1 0} & \mathbf{1 0 4} & \mathbf{. 3 9 0} & \mathbf{1 3 2} \\ \text { OCT } & & & & & & & & \\ 11 \ldots & 1450 & 91.5 & 266 & 83.6 & 1890 & 4100 & \mathbf{1 . 8 4} & 562 \\ \mathbf{1 1} \ldots & \mathbf{1 4 5 0} & \mathbf{9 2 . 7} & \mathbf{2 6 6} & \mathbf{8 6 . 1} & \mathbf{1 8 7 0} & \mathbf{4 1 0 0} & \mathbf{1 . 8 5} & \mathbf{5 6 6}\end{array}$

442509068181901 Northeast Creek monitoring station 104

\begin{tabular}{|c|c|c|c|c|c|c|c|c|}
\hline $\begin{array}{c}\text { MAY } 2000 \\
31 \ldots \\
31 \ldots\end{array}$ & $\begin{array}{l}1215 \\
1215\end{array}$ & $\begin{array}{l}2.62 \\
2.62\end{array}$ & $\begin{array}{l}1.32 \\
1.30\end{array}$ & $\begin{array}{l}.40 \\
.40\end{array}$ & $\begin{array}{l}11.9 \\
11.7\end{array}$ & $\begin{array}{l}19.0 \\
19.0\end{array}$ & $\begin{array}{l}.990 \\
.940\end{array}$ & $\begin{array}{l}3.0 \\
3.0\end{array}$ \\
\hline \multicolumn{9}{|l|}{ JUN } \\
\hline $\begin{array}{l}28 \ldots \\
28 \ldots\end{array}$ & $\begin{array}{l}1025 \\
1025\end{array}$ & $\begin{array}{l}4.48 \\
4.52\end{array}$ & $\begin{array}{l}4.23 \\
4.26\end{array}$ & $\begin{array}{l}1.29 \\
1.30\end{array}$ & $\begin{array}{l}41.9 \\
41.7\end{array}$ & $\begin{array}{l}64.0 \\
64.0\end{array}$ & $\begin{array}{r}1.00 \\
.990\end{array}$ & $\begin{array}{l}6.0 \\
6.0\end{array}$ \\
\hline \multicolumn{9}{|l|}{ AUG } \\
\hline $\begin{array}{l}07 \ldots \\
07 \ldots\end{array}$ & $\begin{array}{l}1515 \\
1515\end{array}$ & $\begin{array}{l}119 \\
116\end{array}$ & $\begin{array}{l}313 \\
312\end{array}$ & $\begin{array}{l}93.2 \\
92.2\end{array}$ & $\begin{array}{l}2960 \\
2950\end{array}$ & $\begin{array}{l}5080 \\
4900\end{array}$ & $\begin{array}{r}.570 \\
.580\end{array}$ & $\begin{array}{l}792 \\
797\end{array}$ \\
\hline $\begin{array}{l}21 \ldots \\
21 \ldots\end{array}$ & $\begin{array}{l}1530 \\
1530\end{array}$ & $\begin{array}{l}90.8 \\
89.6\end{array}$ & $\begin{array}{l}241 \\
236\end{array}$ & $\begin{array}{l}71.6 \\
74.3\end{array}$ & $\begin{array}{l}2140 \\
\mathbf{2 2 0 0}\end{array}$ & $\begin{array}{l}3920 \\
3740\end{array}$ & $\begin{array}{l}.070 \\
.060\end{array}$ & $\begin{array}{l}547 \\
552\end{array}$ \\
\hline \multicolumn{9}{|l|}{ SEP } \\
\hline $\begin{array}{l}25 \ldots \\
25 \ldots\end{array}$ & $\begin{array}{l}1355 \\
1355\end{array}$ & $\begin{array}{l}132 \\
133\end{array}$ & $\begin{array}{l}399 \\
401\end{array}$ & $\begin{array}{l}130 \\
132\end{array}$ & $\begin{array}{l}2970 \\
\mathbf{3 0 0 0}\end{array}$ & $\begin{array}{l}6120 \\
6020\end{array}$ & $\begin{array}{l}.080 \\
.060\end{array}$ & $\begin{array}{l}835 \\
797\end{array}$ \\
\hline
\end{tabular}

442516068175501 Northeast Creek monitoring station 105

$\begin{array}{rrrrrrrrr}\text { JUN } 2000 & & & & & & & \\ 13 \ldots & 1425 & 3.38 & 2.08 & .55 & 18.2 & 31.0 & 1.11 & 4.0 \\ \mathbf{1 3} \ldots & \mathbf{1 4 2 5} & \mathbf{3 . 3 7} & \mathbf{2 . 0 7} & . \mathbf{5 5} & \mathbf{1 8 . 2} & \mathbf{3 1 . 0} & \mathbf{1 . 1 5} & \mathbf{4 . 0} \\ \text { AUG } & & & & & & & & \\ 21 \ldots & 1415 & 40.1 & 105 & 32.8 & 976 & 1630 & \mathbf{. 8 5 0} & 232 \\ \mathbf{2 1} \ldots & \mathbf{1 4 1 5} & \mathbf{3 9 . 3} & \mathbf{1 0 3} & \mathbf{3 2 . 7} & \mathbf{9 7 7} & \mathbf{1 6 4 0} & \mathbf{. 8 5 0} & \mathbf{2 2 6} \\ \mathrm{SEP} & & & & & & & & \\ 25 \ldots & 1310 & 74.3 & 214 & 68.3 & 1820 & -- & .230 & 461 \\ \mathbf{2 5} \ldots & \mathbf{1 3 1 0} & \mathbf{7 5 . 3} & \mathbf{2 1 8} & \mathbf{6 9 . 9} & \mathbf{1 8 2 0} & \mathbf{3 3 0 0} & . \mathbf{2 3 0} & \mathbf{5 3 8} \\ \text { NOV } & & & & & & & & \\ 28 \ldots & 1255 & 2.99 & 1.07 & .92 & 8.73 & 11.0 & 2.69 & 5.0 \\ \mathbf{2 8} \ldots & \mathbf{1 2 5 5} & \mathbf{3 . 0 2} & \mathbf{1 . 0 7} & \mathbf{. 9 2} & \mathbf{8 . 6 5} & \mathbf{1 1 . 0} & \mathbf{2 . 7 3} & \mathbf{5 . 0}\end{array}$


Table A13. --Water-quality assurance records, replicate samples collected May-November 2000, Northeast Creek stations 100 through 105. - Continued.

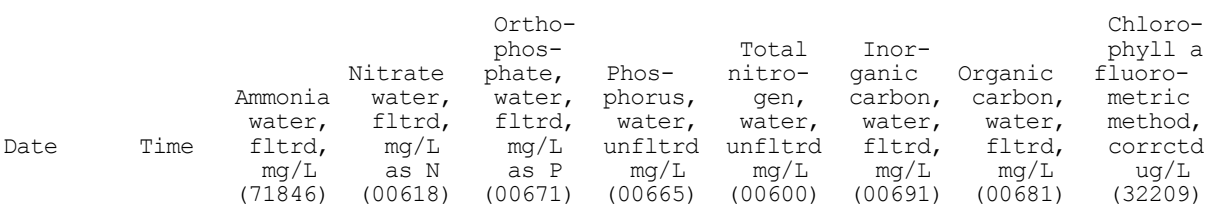

442530068193901 Northeast Creek monitoring station 100

\begin{tabular}{|c|c|c|c|c|c|c|c|}
\hline \multicolumn{8}{|c|}{ JUN 2000} \\
\hline $14 \ldots$ & 1030 & .04 & .00 & 8.10 & 26.0 & .60 & 5.6 \\
\hline $\begin{array}{l}14 \ldots \\
\text { JUL } 2000\end{array}$ & 1030 & -- & -- & - & 26.0 & -- & 5.5 \\
\hline $27 \ldots$ & 0745 & .15 & .00 & 57.0 & 77.0 & .26 & -- \\
\hline $27 \ldots$ & 0745 & .15 & .00 & -- & -- & .28 & - \\
\hline
\end{tabular}

$01022820 \quad$ Northeast Creek monitoring station 101

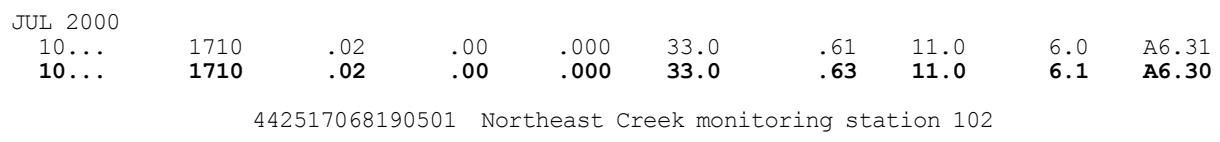

\begin{tabular}{|c|c|c|c|c|c|c|c|c|}
\hline \multicolumn{9}{|l|}{ JUN 2000} \\
\hline $\begin{array}{l}28 \ldots \\
28 \ldots\end{array}$ & $\begin{array}{l}0855 \\
0855\end{array}$ & $\begin{array}{l}.02 \\
.01\end{array}$ & $\begin{array}{l}1.05 \\
1.07\end{array}$ & $\begin{array}{l}7.30 \\
7.30\end{array}$ & $\begin{array}{l}34.0 \\
36.0\end{array}$ & $\begin{array}{l}.74 \\
.73\end{array}$ & $\begin{array}{l}5.9 \\
6.0\end{array}$ & $\begin{array}{l}16.7 \\
16.4\end{array}$ \\
\hline \multicolumn{9}{|l|}{ AUG } \\
\hline $\begin{array}{l}07 \ldots \\
07 \ldots\end{array}$ & $\begin{array}{l}1550 \\
1550\end{array}$ & $\begin{array}{l}.02 \\
.03\end{array}$ & $\begin{array}{l}.00 \\
.00\end{array}$ & $\begin{array}{l}1.50 \\
1.20\end{array}$ & $\begin{array}{l}12.0 \\
11.0\end{array}$ & $\begin{array}{l}.41 \\
.39\end{array}$ & $\begin{array}{l}9.4 \\
9.7\end{array}$ & $\begin{array}{l}2.2 \\
2.2\end{array}$ \\
\hline \multicolumn{9}{|l|}{ SEP } \\
\hline $\begin{array}{l}11 \ldots \\
11 \ldots\end{array}$ & $\begin{array}{l}1625 \\
1625\end{array}$ & $\begin{array}{l}.06 \\
.07\end{array}$ & $\begin{array}{l}18.1 \\
19.1\end{array}$ & $\begin{array}{l}3.30 \\
3.40\end{array}$ & $\begin{array}{l}17.0 \\
18.0\end{array}$ & $\begin{array}{l}.77 \\
.74\end{array}$ & $\begin{array}{l}10.8 \\
11.0\end{array}$ & $\begin{array}{l}2.8 \\
2.8\end{array}$ \\
\hline \multicolumn{9}{|l|}{ NOV } \\
\hline $\begin{array}{l}28 \ldots \\
28 \ldots\end{array}$ & $\begin{array}{l}1535 \\
1535\end{array}$ & $\begin{array}{l}.02 \\
.02\end{array}$ & $\begin{array}{l}.00 \\
.47\end{array}$ & $\begin{array}{l}4.50 \\
\mathbf{4 . 4 0}\end{array}$ & $\begin{array}{l}21.0 \\
20.0\end{array}$ & $\begin{array}{l}.51 \\
.53\end{array}$ & $\begin{array}{l}1.9 \\
2.0\end{array}$ & $\begin{array}{l}14.7 \\
14.9\end{array}$ \\
\hline
\end{tabular}

442507068185301 Northeast Creek monitoring station 103

JUL 2000

$10 \ldots$

10.

$\begin{array}{ll}26 \ldots & 1530 \\ 26 \ldots & 1530\end{array}$

OCT

$11 \ldots$

MAY 2000

MAY 2000

$\begin{array}{ll}31 \ldots & 1215 \\ 31 \ldots & \mathbf{1 2 1 5}\end{array}$

JUN

$28 \ldots \quad 1025$

28... 1025

$07 \ldots 1515$

07... 1515

21... 1530

$21 \ldots \quad 1530$

25... 1355

25... 1355

JUL 2001

$02 \ldots$

1330

1330

442516068175501 Northeast Creek monitoring station 105

JUN 2000

$13 \ldots$

AUG

AUG 21.

$21 \ldots$

SEP

$25 \ldots$

NOV

$28 \ldots$

1425
$\mathbf{1 4 2 5}$

1415

1415

1310

1310

1255
.04

.04

.0

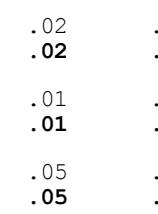

.00
.00
.00
.00
.00
.00

$\begin{array}{rr}.000 & 27.0 \\ -- & -- \\ 4.60 & 21.0 \\ \mathbf{4 . 3 0} & 20.0 \\ 6.40 & 30.0 \\ \mathbf{6 . 9 0} & \mathbf{2 8 . 0}\end{array}$

.77
.76

11.0
11.0

$.55 \quad--$

$--$

$\begin{array}{ll}8.3 & 4.8 \\ 8.5 & 4.8\end{array}$

442509068181901 Northeast Creek monitoring station 104

$\begin{array}{rrrrrrr}.02 & .00 & 2.70 & 18.0 & .52 & 3.5 & 11.2 \\ .02 & .00 & -- & -- & .52 & 3.6 & 10.6 \\ .02 & .00 & 5.10 & 30.0 & .64 & 5.7 & 14.8 \\ .02 & .00 & -- & -- & .66 & 5.5 & 14.9 \\ .01 & .00 & 1.00 & 13.0 & .42 & 6.6 & 3.0 \\ .01 & .00 & -- & -- & .43 & \mathbf{6 . 6} & 3.0 \\ .00 & .00 & .000 & 16.0 & .53 & 5.5 & 3.6 \\ .00 & .00 & .000 & 12.0 & .53 & 5.2 & 3.7 \\ .01 & .00 & 2.20 & 14.0 & .56 & 6.9 & 2.8 \\ .01 & .00 & -- & -.0 & .56 & .5 & \mathbf{2 . 9} \\ <.10 & -- & 2.10 & 21.0 & .54 & -- & -- \\ <.10 & -- & 1.90 & 21.0 & .56 & -- & --\end{array}$

.00

.01

$\begin{array}{ll}.04 & .22 \\ .04 & .22\end{array}$

8.60
.
2.
2.
7.
6.

34.0

.51
.51

$\begin{array}{ll}4.1 & 11.7 \\ 4.0 & 11.6\end{array}$

$.000 \quad 19.0$

$2.50 \quad 19.0$

.55

4.5
4.5

$6.2 \quad$ A1. 60

$\begin{array}{lll}.50 & 5.9 & 3.6 \\ 48 & 6.0 & 3.7\end{array}$

$\begin{array}{lllll}7.90 & 19.0 & .57 & 2.2 & 14.8 \\ 6.70 & 20.0 & .57 & 1.9 & 14.8\end{array}$
A1.96 
Table A14. --Water-quality assurance records, replicate samples collected May-November 2001, Northeast Creek stations 100 through 105. Replicate samples are in bold. [Numbers in parentheses are USGS NWIS parameter code numbers. Abbreviations: fltrd, filtered; mg/L, milligrams per liter; N, nitrogen; P, phosphorus; std, standard; ug/L, micrograms per liter; unfltrd, unfiltered; uS/cm, microsiemens per centimeter; wat unf, unfiltered water; $<$, less than; --, missing data].

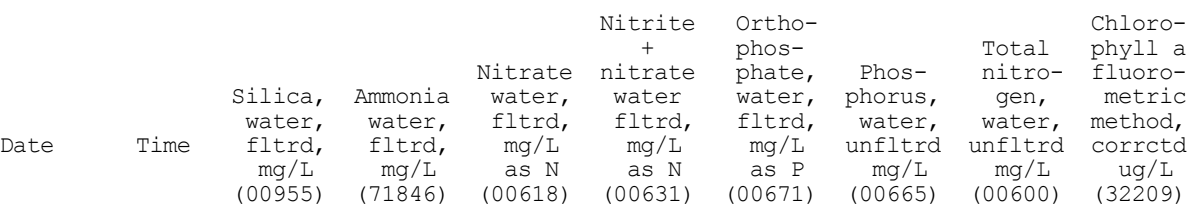

442530068193901 Northeast Creek monitoring station 100

\begin{tabular}{|c|c|c|c|c|c|c|c|c|c|}
\hline $20 \ldots$ & 1140 & .920 & $<.10$ & -- & $<5.00$ & 6.90 & 30.0 & .71 & A2. 32 \\
\hline$\underset{\text { AUG }}{20 \ldots}$ & 1140 & -- & -- & -- & -- & - & -- & -- & A2. 69 \\
\hline $\begin{array}{l}07 \ldots \\
07 \ldots\end{array}$ & $\begin{array}{l}1400 \\
1400\end{array}$ & $\begin{array}{l}<.500 \\
<.500\end{array}$ & $\begin{array}{l}.16 \\
.16\end{array}$ & $\begin{array}{l}-- \\
--\end{array}$ & $\begin{array}{l}<5.00 \\
<5.00\end{array}$ & $\begin{array}{l}25.0 \\
\mathbf{2 8 . 0}\end{array}$ & $\begin{array}{l}72.0 \\
67.0\end{array}$ & $\begin{array}{l}.44 \\
.48\end{array}$ & $\begin{array}{l}\text { A2 } \\
\text { A2. }\end{array}$ \\
\hline
\end{tabular}

01022820 Northeast Creek at Rt 3 bridge, monitoring station 101

MAY 2001

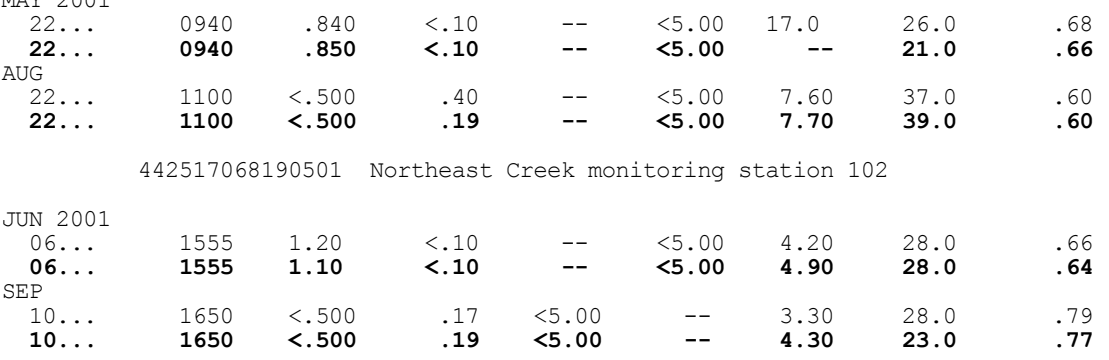

442507068185301 Northeast Creek monitoring station 103

JUN 2001

\begin{tabular}{|c|c|c|c|c|c|c|c|c|}
\hline $\begin{array}{l}20 \ldots \\
20 \ldots \\
\text { DCT }\end{array}$ & $\begin{array}{l}1600 \\
1600\end{array}$ & $\begin{array}{l}1.00 \\
1.00\end{array}$ & $\begin{array}{l}<.10 \\
<.10\end{array}$ & $\begin{array}{l}-- \\
--\end{array}$ & $\begin{array}{l}<5.00 \\
<5.00\end{array}$ & $\begin{array}{r}8.00 \\
--\end{array}$ & $\begin{array}{l}36.0 \\
37.0\end{array}$ & $\begin{array}{l}.70 \\
.69\end{array}$ \\
\hline $\begin{array}{l}01 \ldots \\
01 \ldots\end{array}$ & $\begin{array}{l}1450 \\
1450\end{array}$ & $\begin{array}{l}<.500 \\
<.500\end{array}$ & $\begin{array}{l}.17 \\
.18\end{array}$ & $\begin{array}{l}-- \\
--\end{array}$ & $\begin{array}{l}<5.00 \\
<5.00\end{array}$ & $\begin{array}{l}1.40 \\
2.00\end{array}$ & $\begin{array}{l}12.0 \\
12.0\end{array}$ & $\begin{array}{l}.66 \\
.66\end{array}$ \\
\hline $\begin{array}{l}06 \ldots \\
06 \ldots\end{array}$ & $\begin{array}{l}1500 \\
1500\end{array}$ & $\begin{array}{l}<.500 \\
<.500\end{array}$ & $\begin{array}{l}.23 \\
.10\end{array}$ & $\begin{array}{l}-- \\
--\end{array}$ & $\begin{array}{l}<5.00 \\
<5.00\end{array}$ & $\begin{array}{l}3.40 \\
2.60\end{array}$ & $\begin{array}{l}35.0 \\
34.0\end{array}$ & .4 \\
\hline
\end{tabular}

442509068181901 Northeast Creek monitoring station 104

JUL 2001

$02 \ldots$

$\begin{array}{llllllll}1330 & <.500 & <.10 & -- & <5.00 & 2.10 & 21.0 & .54 \\ 1330< & <.500 & <.10 & -- & <5.00 & \mathbf{1 . 9 0} & \mathbf{2 1 . 0} & .56\end{array}$

442516068175501 Northeast Creek monitoring station 105

JUL 2001

$02 \ldots$

$02 \ldots$

$18 \ldots$

$18 \ldots$

1230
1230
1320

1.20
--
.800
.790

$<.10$
--
$<.10$
$<.10$

--
--
--
--

$<5.00$
--
$<5.00$
$<5.00$

2.50
--
6.20
5.60

27.0

--
28.0

.53
--
.60
.58 


\section{Appendix 2}

Water temperature and specific conductance monitored at two depths, stations 101-105 
Table A15. Specific conductance and water temperature data collected May-November 2000 at station 101 (USGS identifier 01022820) Northeast Creek at Route 3 bridge near Bar Harbor, ME [Data are presented as minimum, mean and maximum daily values of continuous data at 15-minute intervals. Shallow and deep data were collected at fixed depths located, respectively, about $0.2 \mathrm{~m}$ below the water surface and about $0.2 \mathrm{~m}$ above the stream bottom.]

SHALLOW

Specific conductance, water, microsiemens per centimeter at 25 degrees Celsius

\begin{tabular}{|c|c|c|c|c|c|c|c|c|c|c|c|c|}
\hline \multirow[t]{2}{*}{ DAY } & MAX & MIN & MEAN & MAX & MIN & MEAN & MAX & MIN & MEAN & MAX & MIN & MEAN \\
\hline & & MAY & & \multicolumn{3}{|c|}{ JUNE } & \multicolumn{3}{|c|}{ JULY } & & \multicolumn{2}{|l|}{ AUGUST } \\
\hline 1 & --- & --- & --- & --- & --- & --- & --- & --- & --- & --- & --- & \\
\hline 2 & --- & --- & --- & --- & --- & --- & --- & --- & --- & --- & --- & \\
\hline $\begin{array}{l}3 \\
4\end{array}$ & --- & --- & $\begin{array}{l}--- \\
---\end{array}$ & 44800 & --- & $311^{---}$ & --- & --- & --- & --- & --- & \\
\hline 5 & --- & --- & --- & 44400 & 15100 & 28000 & --- & --- & --- & --- & -- & \\
\hline 6 & --- & --- & --- & 41500 & 11800 & 25100 & --- & --- & --- & --- & --- & \\
\hline 7 & --- & --- & --- & 43400 & 7990 & 23800 & 40000 & 20500 & 29400 & --- & --- & \\
\hline 8 & --- & --- & --- & 19200 & 3340 & 7440 & 26200 & 13500 & 17800 & 33600 & 30500 & 31400 \\
\hline 9 & --- & --- & --- & 10400 & 2350 & 3610 & 26000 & 10800 & 13200 & 38200 & 28100 & 31000 \\
\hline 10 & & & & --- & --- & --- & --- & --- & --- & 37200 & & \\
\hline 11 & --- & --- & --- & --- & --- & --- & --- & --- & --- & 33200 & 27500 & \\
\hline 12 & --- & --- & --- & --- & --- & --- & --- & --- & --- & 39100 & 27000 & \\
\hline 13 & --- & --- & --- & --- & --- & --- & --- & --- & --- & 47100 & 28200 & \\
\hline 14 & --- & -- & --- & --- & --- & --- & --- & --- & --- & 47100 & 37200 & \\
\hline 15 & --- & --- & --- & --- & --- & --- & --- & --- & --- & 47600 & 40000 & 44300 \\
\hline 16 & --- & --- & --- & --- & --- & --- & --- & --- & --- & 47400 & 40800 & \\
\hline 17 & 7000 & 283 & 997 & --- & --- & --- & --- & --- & --- & 46800 & 40600 & \\
\hline 18 & 7000 & 493 & 1570 & --- & --- & --- & --- & --- & --- & 46500 & 38100 & \\
\hline 19 & 7000 & 303 & 1620 & 38200 & 3070 & 13300 & --- & --- & --- & 46800 & 38800 & 7 \\
\hline 20 & 5080 & 187 & 485 & 17900 & 2560 & 11500 & --- & --- & --- & 45500 & 38900 & 4 \\
\hline 21 & 2350 & 210 & 300 & 14400 & 1930 & 4940 & --- & --- & --- & 38900 & 33600 & \\
\hline & 284 & 228 & 26 & & & & --- & --- & --- & 39500 & 31700 & \\
\hline 23 & 354 & 284 & 331 & 14600 & 2830 & 8510 & --- & --- & --- & 40900 & 30600 & \\
\hline 24 & 360 & 242 & 318 & 3330 & 2090 & 2610 & --- & --- & --- & 48200 & 30700 & 353 \\
\hline 25 & 254 & 86 & 163 & 11100 & 1950 & 3240 & --- & --- & --- & 50400 & 38800 & 4 \\
\hline 26 & 92 & 78 & 84 & 11800 & 2280 & 3980 & --- & --- & --- & 50500 & 45900 & \\
\hline 7 & 118 & 90 & 102 & 34200 & 2390 & 9580 & --- & --- & --- & 50400 & 46700 & \\
\hline 28 & 130 & 118 & 122 & 34100 & 12400 & 23200 & --- & --- & --- & 50200 & 48000 & \\
\hline 29 & 152 & 130 & 140 & --- & --- & --- & --- & --- & --- & 51100 & 48200 & \\
\hline 30 & 3950 & 152 & 331 & --- & --- & --- & --- & --- & --- & 51100 & 48600 & 4 \\
\hline 31 & & - & & --- & --- & --- & --- & --- & --- & 51000 & 48700 & \\
\hline $\mathrm{MC}$ & & & & 44800 & 1930 & 13300 & & & & 51100 & 27000 & \\
\hline
\end{tabular}

\begin{tabular}{|c|c|c|c|c|c|c|c|c|c|c|c|c|}
\hline \multirow[t]{2}{*}{ DAY } & MAX & MIN & MEAN & MAX & MIN & MEAN & MAX & MIN & MEAN & MAX & MIN & MEAN \\
\hline & \multicolumn{3}{|c|}{ SEPTEMBER } & \multicolumn{3}{|c|}{ OCTOBER } & \multicolumn{3}{|c|}{ NOVEMBER } & \multicolumn{3}{|c|}{ DECEMBER } \\
\hline $\begin{array}{l}1 \\
2 \\
3 \\
4 \\
5\end{array}$ & $\begin{array}{l}50900 \\
49800 \\
48600 \\
46600 \\
43200\end{array}$ & $\begin{array}{l}47500 \\
46700 \\
45300 \\
42100 \\
38500\end{array}$ & $\begin{array}{l}49300 \\
48400 \\
47100 \\
44100 \\
40600\end{array}$ & $\begin{array}{l}49500 \\
47900 \\
48600 \\
45400 \\
41600\end{array}$ & $\begin{array}{l}41200 \\
38200 \\
25000 \\
23300 \\
25300\end{array}$ & $\begin{array}{l}45600 \\
43700 \\
40100 \\
37900 \\
39200\end{array}$ & $\begin{array}{r}3720 \\
1330 \\
698 \\
607 \\
703\end{array}$ & $\begin{array}{r}1330 \\
690 \\
572 \\
571 \\
607\end{array}$ & $\begin{array}{r}2330 \\
927 \\
619 \\
581 \\
645\end{array}$ & $\begin{array}{l}128 \\
158 \\
202 \\
262 \\
324\end{array}$ & $\begin{array}{l}112 \\
127 \\
157 \\
202 \\
262\end{array}$ & $\begin{array}{l}121 \\
141 \\
177 \\
230 \\
296\end{array}$ \\
\hline $\begin{array}{r}6 \\
7 \\
8 \\
9 \\
10\end{array}$ & $\begin{array}{l}39600 \\
38700 \\
37200 \\
37700 \\
41300\end{array}$ & $\begin{array}{l}35600 \\
34100 \\
34000 \\
34400 \\
33300\end{array}$ & $\begin{array}{l}38000 \\
36000 \\
35700 \\
36200 \\
34300\end{array}$ & $\begin{array}{l}40700 \\
33200 \\
26400 \\
28900 \\
29200\end{array}$ & $\begin{array}{l}27500 \\
21000 \\
19100 \\
18300 \\
11400\end{array}$ & $\begin{array}{l}32700 \\
28100 \\
21300 \\
20800 \\
17300\end{array}$ & $\begin{array}{r}763 \\
758 \\
718 \\
16000 \\
36500\end{array}$ & $\begin{array}{l}703 \\
712 \\
677 \\
693 \\
889\end{array}$ & $\begin{array}{r}749 \\
739 \\
692 \\
2680 \\
10400\end{array}$ & $\begin{array}{r}390 \\
399 \\
463 \\
29200 \\
31700\end{array}$ & $\begin{array}{l}324 \\
386 \\
398 \\
461 \\
592\end{array}$ & $\begin{array}{r}363 \\
390 \\
431 \\
2910 \\
5080\end{array}$ \\
\hline $\begin{array}{l}11 \\
12 \\
13 \\
14 \\
15\end{array}$ & $\begin{array}{l}35800 \\
45500 \\
45800 \\
48000 \\
49200\end{array}$ & $\begin{array}{l}33100 \\
32800 \\
37900 \\
41900 \\
39600\end{array}$ & $\begin{array}{l}33800 \\
34300 \\
41500 \\
45100 \\
45000\end{array}$ & $\begin{array}{l}20200 \\
18800 \\
29800 \\
44400 \\
43400\end{array}$ & $\begin{array}{r}7390 \\
6670 \\
6890 \\
8040 \\
27200\end{array}$ & $\begin{array}{r}11200 \\
7610 \\
12800 \\
26400 \\
37300\end{array}$ & $\begin{array}{l}42800 \\
43200 \\
46000 \\
50100 \\
42600\end{array}$ & $\begin{array}{r}1600 \\
2500 \\
2180 \\
3500 \\
10200\end{array}$ & $\begin{array}{l}17600 \\
17200 \\
20500 \\
27600 \\
24500\end{array}$ & $\begin{array}{l}58100 \\
54600 \\
43600 \\
57200 \\
20000\end{array}$ & $\begin{array}{r}708 \\
5930 \\
11600 \\
4910 \\
3840\end{array}$ & $\begin{array}{r}30300 \\
42300 \\
25200 \\
24800 \\
8530\end{array}$ \\
\hline $\begin{array}{l}16 \\
17 \\
18 \\
19 \\
20\end{array}$ & $\begin{array}{l}47900 \\
47300 \\
44700 \\
45100 \\
47000\end{array}$ & $\begin{array}{l}39400 \\
42200 \\
38800 \\
36200 \\
35300\end{array}$ & $\begin{array}{l}45200 \\
45100 \\
41800 \\
40100 \\
40900\end{array}$ & $\begin{array}{l}42700 \\
46300 \\
49200 \\
38100 \\
28300\end{array}$ & $\begin{array}{r}14300 \\
18300 \\
13400 \\
25500 \\
5430\end{array}$ & $\begin{array}{l}29400 \\
33900 \\
31900 \\
33300 \\
15200\end{array}$ & $\begin{array}{r}12500 \\
23900 \\
1610 \\
815 \\
13800\end{array}$ & $\begin{array}{r}1550 \\
979 \\
800 \\
572 \\
577\end{array}$ & $\begin{array}{r}4620 \\
3990 \\
1030 \\
642 \\
1710\end{array}$ & $\begin{array}{r}5220 \\
19900 \\
1440 \\
199 \\
474\end{array}$ & $\begin{array}{r}2330 \\
1440 \\
195 \\
146 \\
139\end{array}$ & $\begin{array}{r}2900 \\
4030 \\
491 \\
160 \\
160\end{array}$ \\
\hline $\begin{array}{l}21 \\
22 \\
23 \\
24 \\
25\end{array}$ & $\begin{array}{l}49200 \\
48700 \\
49400 \\
48600 \\
49100\end{array}$ & $\begin{array}{l}35700 \\
42500 \\
38300 \\
41400 \\
41400\end{array}$ & $\begin{array}{l}42200 \\
46100 \\
43300 \\
45500 \\
45800\end{array}$ & $\begin{array}{l}34400 \\
33000 \\
31300 \\
34000 \\
46800\end{array}$ & $\begin{array}{r}4200 \\
4610 \\
3740 \\
3740 \\
16900\end{array}$ & $\begin{array}{r}11600 \\
15300 \\
7180 \\
15300 \\
31400\end{array}$ & $\begin{array}{r}7440 \\
18900 \\
13800 \\
23100 \\
25800\end{array}$ & $\begin{array}{l}697 \\
617 \\
500 \\
432 \\
486\end{array}$ & $\begin{array}{l}1010 \\
2780 \\
2060 \\
3210 \\
4610\end{array}$ & $\begin{array}{l}203 \\
151 \\
178 \\
237 \\
351\end{array}$ & $\begin{array}{l}144 \\
142 \\
149 \\
177 \\
237\end{array}$ & $\begin{array}{l}171 \\
146 \\
161 \\
203 \\
290\end{array}$ \\
\hline $\begin{array}{l}26 \\
27 \\
28 \\
29 \\
30 \\
31\end{array}$ & $\begin{array}{r}50800 \\
52100 \\
52100 \\
50900 \\
50600 \\
---\end{array}$ & $\begin{array}{l}42800 \\
47000 \\
46100 \\
45600 \\
41500\end{array}$ & $\begin{array}{r}47900 \\
49300 \\
49300 \\
48500 \\
47000 \\
---\end{array}$ & $\begin{array}{l}45400 \\
49700 \\
53500 \\
51500 \\
44200 \\
23000\end{array}$ & $\begin{array}{r}30600 \\
29100 \\
36900 \\
31100 \\
17300 \\
3650\end{array}$ & $\begin{array}{l}39800 \\
41000 \\
47000 \\
43100 \\
31200 \\
10900\end{array}$ & $\begin{array}{r}38200 \\
3680 \\
188 \\
108 \\
113 \\
---\end{array}$ & $\begin{array}{l}625 \\
186 \\
108 \\
101 \\
103 \\
---\end{array}$ & $\begin{array}{r}10200 \\
1170 \\
132 \\
104 \\
107 \\
---\end{array}$ & $\begin{array}{r}491 \\
542 \\
19300 \\
653 \\
1410 \\
13600\end{array}$ & $\begin{array}{l}351 \\
491 \\
532 \\
529 \\
555 \\
479\end{array}$ & $\begin{array}{r}429 \\
522 \\
1880 \\
568 \\
588 \\
848\end{array}$ \\
\hline NT & 52100 & 2800 & 42900 & 53500 & 3650 & 27700 & 50100 & 101 & 5500 & 58100 & 112 & 49 \\
\hline
\end{tabular}


Table A15. Specific conductance and water temperature data collected May-November 2000 at station 101 (USGS identifier 01022820) Northeast Creek at Route 3 bridge near Bar Harbor, ME - Continued.

DEEP

Specific conductance, water, microsiemens per centimeter at 25 degrees Celsius

\begin{tabular}{|c|c|c|c|c|c|c|c|c|c|c|c|c|}
\hline DAY & MAX & $\begin{array}{l}\text { MIN } \\
\text { MAY }\end{array}$ & MEAN & MAX & \multicolumn{2}{|l|}{ JUNE } & \multicolumn{2}{|r|}{ JULY } & MEAN & MAX & \multicolumn{2}{|l|}{ AUGUST } \\
\hline 1 & --- & --- & --- & --- & --- & --- & 47900 & 35400 & 44100 & --- & --- & - \\
\hline 2 & --- & --- & --- & --- & --- & --- & 48200 & 36700 & 45300 & --- & --- & \\
\hline 3 & --- & --- & --- & --- & --- & --- & 49000 & 37800 & 46200 & -- & -- & --- \\
\hline $\begin{array}{l}4 \\
5\end{array}$ & $\begin{array}{l}--- \\
---\end{array}$ & $\begin{array}{l}--- \\
---\end{array}$ & $\begin{array}{l}--- \\
---\end{array}$ & $\begin{array}{l}45200 \\
44600\end{array}$ & $\begin{array}{l}17300 \\
17300\end{array}$ & $\begin{array}{l}36100 \\
36000\end{array}$ & $\begin{array}{l}48200 \\
47700\end{array}$ & $\begin{array}{l}40000 \\
37800\end{array}$ & $\begin{array}{l}45400 \\
44200\end{array}$ & $\begin{array}{l}--- \\
---\end{array}$ & $\begin{array}{l}--- \\
---\end{array}$ & $\begin{array}{l}--- \\
---\end{array}$ \\
\hline 6 & --- & --- & --- & 42100 & 18400 & 32800 & 46400 & 37000 & 42000 & --- & --- & - \\
\hline 7 & --- & --- & --- & 44100 & 17500 & 35000 & 39900 & 29800 & 35300 & --- & --- & \\
\hline 8 & --- & --- & --- & 27100 & 4250 & 14500 & 32000 & 21900 & 26600 & 41500 & $\begin{array}{l}39900 \\
397\end{array}$ & 40600 \\
\hline 9 & --- & --- & --- & 12300 & 2460 & 5720 & 28900 & 22300 & 25000 & 41100 & 39700 & 40300 \\
\hline 10 & --- & --- & --- & 10800 & 1630 & 3310 & --- & --- & --- & 41700 & 20200 & 38200 \\
\hline 11 & --- & --- & --- & 14200 & 1500 & 5300 & --- & --- & --- & 41200 & 38400 & 39800 \\
\hline 12 & --- & --- & --- & 12400 & 3900 & 9470 & --- & --- & --- & 43700 & 38600 & 39500 \\
\hline 13 & --- & --- & --- & 17400 & 8200 & 10800 & --- & --- & --- & 46400 & 42300 & 43400 \\
\hline $\begin{array}{l}14 \\
15\end{array}$ & --- & --- & --- & 23000 & 11300 & 15900 & --- & --- & --- & 47300 & 44700 & 46500 \\
\hline 15 & --- & --- & --- & 31000 & 19400 & 22300 & --- & --- & --- & 47700 & 44700 & 47000 \\
\hline 16 & --- & --- & --- & 31900 & 23200 & 30700 & --- & --- & --- & 48000 & 45300 & 47100 \\
\hline 17 & 7000 & 300 & 3790 & 33500 & 27700 & 32500 & --- & --- & --- & 47400 & 44500 & 46500 \\
\hline 18 & 7000 & 506 & 3990 & 30600 & 24600 & 27300 & --- & --- & --- & 47700 & 45100 & 46900 \\
\hline $\begin{array}{l}19 \\
20\end{array}$ & $\begin{array}{l}7000 \\
7000\end{array}$ & $\begin{array}{l}29 y \\
186\end{array}$ & $\begin{array}{r}2860 \\
872\end{array}$ & $\begin{array}{l}28000 \\
23900\end{array}$ & $\begin{array}{l}22200 \\
21600\end{array}$ & $\begin{array}{l}23900 \\
22600\end{array}$ & $\begin{array}{l}--- \\
---\end{array}$ & --- & $\begin{array}{l}--- \\
---\end{array}$ & 47600 & $\begin{array}{l}45300 \\
44700\end{array}$ & $\begin{array}{l}46800 \\
46000\end{array}$ \\
\hline 21 & 3940 & 212 & 451 & 22800 & 22000 & 22500 & --- & --- & --- & 0 & 44700 & 0 \\
\hline 22 & 290 & 230 & 268 & 23300 & 22000 & 22600 & --- & --- & --- & 45900 & 43700 & 44900 \\
\hline 23 & 486 & 289 & 342 & 22400 & 21300 & 21800 & --- & --- & --- & 45500 & 43100 & 44500 \\
\hline 24 & 365 & 245 & 324 & 21500 & 20400 & 21100 & --- & --- & --- & 49000 & 43900 & 45700 \\
\hline 25 & 260 & 87 & 165 & 20800 & 19400 & 20300 & --- & --- & --- & 50400 & 46800 & 48500 \\
\hline 26 & 93 & 79 & 84 & 22600 & 19100 & 20200 & --- & --- & --- & 50500 & 47300 & 49600 \\
\hline 27 & 120 & 91 & 104 & 38800 & 21700 & 25300 & --- & --- & --- & 50800 & 47500 & 49600 \\
\hline 28 & 139 & 119 & 124 & 45400 & 32500 & 38400 & --- & --- & --- & 50300 & 48200 & 49200 \\
\hline 29 & 154 & 132 & 142 & 47900 & 33600 & 41800 & --- & --- & --- & 50300 & 48600 & 49600 \\
\hline $\begin{array}{l}30 \\
31\end{array}$ & 6440 & 154 & 689 & 47800 & 33900 & 44700 & --- & --- & --- & 50500 & 48900 & 49600 \\
\hline & & & & & & & & & & & & \\
\hline MONTH & 7000 & 79 & 1010 & 47900 & 1500 & 23800 & 49000 & 21900 & 39300 & 50800 & 20200 & 45600 \\
\hline \multirow[t]{2}{*}{ DAY } & MAX & MIN & MEAN & MAX & MIN & MEAN & MAX & MIN & MEAN & MAX & MIN & MEAN \\
\hline & \multicolumn{3}{|c|}{ SEPTEMBER } & \multicolumn{3}{|c|}{ OCTOBER } & \multicolumn{3}{|c|}{ NOVEMBER } & \multicolumn{3}{|c|}{ DECEMBER } \\
\hline 1 & 50600 & 48500 & 49500 & 48100 & 44200 & 46700 & 25500 & 1340 & 9360 & 135 & 116 & 126 \\
\hline $\begin{array}{l}2 \\
3\end{array}$ & $\begin{array}{l}50400 \\
49800\end{array}$ & $\begin{array}{l}48100 \\
25900\end{array}$ & $\begin{array}{l}49200 \\
42000\end{array}$ & $\begin{array}{l}47500 \\
47800\end{array}$ & $\begin{array}{l}44700 \\
21700\end{array}$ & $\begin{array}{l}46500 \\
39400\end{array}$ & $\begin{array}{r}1340 \\
714\end{array}$ & $\begin{array}{l}699 \\
580\end{array}$ & $\begin{array}{l}938 \\
629\end{array}$ & $\begin{array}{l}163 \\
209\end{array}$ & $\begin{array}{l}130 \\
163\end{array}$ & $\begin{array}{l}147 \\
183\end{array}$ \\
\hline 4 & & 21500 & 38300 & 47400 & 24400 & 44900 & 629 & 580 & 589 & 267 & 209 & 235 \\
\hline 5 & 44100 & 21900 & 31300 & 46400 & 23000 & 35000 & 719 & 621 & 651 & 331 & 267 & 301 \\
\hline 6 & 44900 & 29000 & 41600 & 45400 & 22800 & 31800 & 776 & 719 & 759 & 397 & 331 & \\
\hline 8 & $\begin{array}{l}44800 \\
44500\end{array}$ & $\begin{array}{l}28200 \\
23200\end{array}$ & $\begin{array}{l}40700 \\
39300\end{array}$ & $\begin{array}{l}44800 \\
43300\end{array}$ & $\begin{array}{l}42800 \\
41600\end{array}$ & $\begin{array}{l}43800 \\
42600\end{array}$ & $\begin{array}{l}768 \\
730\end{array}$ & $\begin{array}{l}724 \\
689\end{array}$ & $\begin{array}{l}751 \\
703\end{array}$ & $\begin{array}{l}410 \\
477\end{array}$ & $\begin{array}{l}393 \\
410\end{array}$ & \\
\hline & 44600 & 23200 & 38400 & 42300 & 36200 & 40900 & 19600 & 703 & 11900 & 29000 & 477 & 18400 \\
\hline 10 & 44000 & 20900 & 36500 & 37700 & 36800 & 37300 & 35300 & 9860 & 24800 & 31600 & 25400 & 29000 \\
\hline 11 & 43300 & 18700 & 32500 & 37600 & 36300 & 37000 & 39200 & 12500 & 30200 & 48100 & 21600 & 38900 \\
\hline $\begin{array}{l}12 \\
13\end{array}$ & $\begin{array}{l}43100 \\
48400\end{array}$ & $\begin{array}{l}22000 \\
25200\end{array}$ & $\begin{array}{l}30700 \\
41800\end{array}$ & $\begin{array}{l}36600 \\
32300\end{array}$ & $\begin{array}{l}31300 \\
29100\end{array}$ & $\begin{array}{l}35500 \\
30800\end{array}$ & $\begin{array}{l}37900 \\
40900\end{array}$ & $\begin{array}{r}7850 \\
14200\end{array}$ & $\begin{array}{l}28900 \\
31200\end{array}$ & $\begin{array}{l}47800 \\
40700\end{array}$ & $\begin{array}{l}22700 \\
16600\end{array}$ & $\begin{array}{l}41300 \\
33500\end{array}$ \\
\hline 14 & 48200 & 23200 & 43300 & 42100 & 30400 & 37000 & 44000 & 18700 & 35800 & 47000 & 14000 & 36100 \\
\hline 15 & 50100 & 44400 & 49200 & 40900 & 36200 & 39300 & 42700 & 15900 & 32900 & 41200 & 14400 & 29500 \\
\hline 16 & 48000 & 28100 & 39900 & 42700 & 35300 & 400 & 33200 & 1570 & 19600 & 1 & 2640 & 10600 \\
\hline $\begin{array}{l}17 \\
18\end{array}$ & $\begin{array}{l}48100 \\
47600\end{array}$ & $\begin{array}{l}21900 \\
23700\end{array}$ & $\begin{array}{l}39600 \\
35300\end{array}$ & $\begin{array}{l}42800 \\
46300\end{array}$ & $\begin{array}{l}37500 \\
36800\end{array}$ & $\begin{array}{l}41700 \\
43400\end{array}$ & $\begin{array}{l}24900 \\
22300\end{array}$ & 1000 & $\begin{array}{r}9490 \\
17200\end{array}$ & 22500 & $\begin{array}{r}1460 \\
200\end{array}$ & $\begin{array}{r}9880 \\
502\end{array}$ \\
\hline 19 & 47400 & 23200 & 35500 & 46200 & 31700 & 41300 & 11800 & 691 & 5530 & 204 & & \\
\hline 20 & 300 & 22900 & 3720 & 36200 & 31500 & 35500 & 19000 & 7030 & 12900 & 491 & 145 & 66 \\
\hline 21 & 49300 & 45700 & 47900 & 36100 & 28000 & 34900 & 17700 & 13800 & 15000 & 209 & 150 & 6 \\
\hline $\begin{array}{l}22 \\
23\end{array}$ & $\begin{array}{l}49300 \\
49100\end{array}$ & $\begin{array}{l}22000 \\
22800\end{array}$ & $\begin{array}{l}35800 \\
32400\end{array}$ & $\begin{array}{l}33900 \\
33500\end{array}$ & $\begin{array}{l}32400 \\
29700\end{array}$ & $\begin{array}{l}33400 \\
32800\end{array}$ & $\begin{array}{l}19500 \\
16900\end{array}$ & $\begin{array}{l}8880 \\
9060\end{array}$ & $\begin{array}{l}16400 \\
13400\end{array}$ & $\begin{array}{l}157 \\
184\end{array}$ & $\begin{array}{l}147 \\
155\end{array}$ & $\begin{array}{l}152 \\
167\end{array}$ \\
\hline 24 & 00 & 23700 & 38400 & 年 & 29500 & 314 & 23400 & 3150 & 16600 & & & \\
\hline 25 & 49600 & 44700 & 47300 & 41600 & 31900 & 37000 & 25700 & 15600 & 22800 & 360 & 244 & 77 \\
\hline 26 & 50200 & 46300 & 48900 & 42600 & 35600 & 41400 & 34900 & 19500 & 29400 & 497 & 360 & \\
\hline $\begin{array}{l}27 \\
28\end{array}$ & $\begin{array}{l}50200 \\
50200\end{array}$ & $\begin{array}{l}47200 \\
46300\end{array}$ & $\begin{array}{l}49000 \\
48800\end{array}$ & $\begin{array}{l}45800 \\
46500\end{array}$ & $\begin{array}{l}34600 \\
39900\end{array}$ & 44000 & $\begin{array}{r}34200 \\
192\end{array}$ & $\begin{array}{l}192 \\
110\end{array}$ & $\begin{array}{r}15100 \\
136\end{array}$ & $\begin{array}{r}551 \\
23700\end{array}$ & $\begin{array}{l}491 \\
543\end{array}$ & 10100 \\
\hline 29 & 49200 & 45600 & 48100 & 45100 & 38400 & 43800 & 114 & 105 & 108 & 17800 & 548 & 2680 \\
\hline 30 & 49100 & 44600 & 47600 & 43900 & 31800 & 39100 & 120 & 107 & 112 & 11700 & 569 & 2170 \\
\hline 31 & & & & 391 & 1970 & 31500 & - & --- & & 13600 & 498 & 2430 \\
\hline MONTH & 50600 & 18700 & 41200 & 48100 & 19700 & 38800 & 44000 & 105 & 13500 & 48100 & 116 & 8700 \\
\hline
\end{tabular}


Table A15. Specific conductance and water temperature data collected May-November 2000 at station 101 (USGS identifier 01022820) Northeast Creek at Route 3 bridge near Bar Harbor, ME - Continued.

SHALLOW

Temperature, water, degrees Celsius

\begin{tabular}{|c|c|c|c|c|c|c|c|c|c|c|c|c|}
\hline \multirow[t]{2}{*}{ DAY } & MAX & MIN & MEAN & MAX & MIN & MEAN & MAX & MIN & MEAN & MAX & MIN & MEAN \\
\hline & & MAY & & \multicolumn{3}{|c|}{ JUNE } & \multicolumn{3}{|c|}{ JULY } & \multicolumn{3}{|c|}{ AUGUST } \\
\hline 1 & --- & --- & --- & --- & --- & --- & --- & --- & --- & --- & --- & --- \\
\hline 2 & --- & --- & --- & --- & --- & --- & --- & --- & --- & --- & --- & --- \\
\hline 3 & --- & --- & --- & --- & --- & --- & --- & --- & --- & --- & --- & --- \\
\hline $\begin{array}{l}4 \\
5\end{array}$ & $\begin{array}{l}--- \\
---\end{array}$ & $\begin{array}{l}--- \\
---\end{array}$ & $\begin{array}{l}--- \\
---\end{array}$ & 20.2 & $\begin{array}{r}13.9 \\
12.5\end{array}$ & $\begin{array}{l}17.2 \\
18.0\end{array}$ & --- & --- & $\begin{array}{l}--- \\
---\end{array}$ & --- & --- & --- \\
\hline & & & & & & & & & & & & \\
\hline 6 & --- & --- & --- & 20.1 & 12.7 & 16.6 & 25.3 & $\begin{array}{l}11.0 \\
19.8\end{array}$ & 18.4 & --- & --- & \\
\hline 8 & --- & -- & -- & 19.2 & 12.7 & 16.2 & 23.3 & 20.1 & 21.9 & 29.5 & 21.9 & 25.2 \\
\hline 9 & --- & --- & --- & 22.2 & 15.7 & 19.1 & 27.1 & 18.6 & 22.2 & 26.6 & 22.2 & \\
\hline 10 & --- & --- & --- & -- & -- & -- & -- & -- & -- & 28.5 & 23.0 & 25.0 \\
\hline 11 & --- & --- & --- & --- & --- & --- & --- & --- & --- & 28.5 & 19.8 & 24.0 \\
\hline 11 & --- & --- & --- & --- & --- & --- & --- & --- & --- & 25.3 & 19.5 & \\
\hline 13 & --- & --- & --- & --- & --- & -- & --- & --- & --- & 24.1 & 20.2 & \\
\hline 14 & --- & --- & --- & --- & --- & --- & --- & --- & --- & 22.6 & 18.9 & 21.4 \\
\hline 15 & --- & --- & --- & --- & --- & --- & --- & --- & --- & 23.3 & 18.6 & 21.2 \\
\hline 16 & --- & --- & --- & --_ & --- & --- & --_ & --- & --_ & 22.6 & 18.6 & 21.0 \\
\hline 17 & 18.6 & 13.9 & 15.9 & --- & --- & --- & --- & --- & --- & 24.1 & 18.6 & \\
\hline 18 & 15.4 & 13.7 & 14.6 & --- & --- & --- & --- & --- & --- & 24.5 & 17.7 & \\
\hline 19 & 14.7 & 12.3 & 13.6 & --- & --- & --- & --- & --- & --- & 23.7 & 19.2 & \\
\hline 20 & 17.4 & 11.8 & 14.7 & --- & --- & --- & --- & --- & --- & 22.6 & 17.4 & 19.5 \\
\hline 21 & 17.1 & 14.9 & 15.9 & --- & --- & --- & --- & --- & --- & 24.1 & 15.7 & 19.7 \\
\hline 22 & 18.6 & 14.7 & 16.5 & --- & --- & --- & --- & --- & --- & 25.8 & 16.5 & \\
\hline 23 & 17.1 & 14.2 & 15.4 & --- & --- & --- & --- & --- & --- & 24.5 & 18.6 & \\
\hline 24 & 14.2 & 11.6 & 12.9 & --- & --- & --- & --- & --- & --- & 26.6 & 18.9 & 22.3 \\
\hline 25 & 11.6 & 10.9 & 11.4 & --- & --- & --- & --- & --- & --- & 26.6 & 19.8 & 22.7 \\
\hline 26 & 16.6 & 10.5 & 13.3 & --- & --- & --- & --- & --- & --- & 28.0 & 20.2 & \\
\hline 27 & 17.1 & 14.2 & 15.8 & --- & --- & --- & --- & --- & --- & 25.3 & 18.0 & \\
\hline $\begin{array}{ll}28 \\
29\end{array}$ & 16.3 & 14.4 & 15.2 & -- & -- & --- & --- & --- & -- & 22.6 & 17.4 & $\begin{array}{l}19.7 \\
18.8\end{array}$ \\
\hline $\begin{array}{l}29 \\
30\end{array}$ & $\begin{array}{l}15.2 \\
19.8\end{array}$ & $\begin{array}{l}13.9 \\
12.9\end{array}$ & $\begin{array}{l}14.5 \\
16.0\end{array}$ & --- & --- & ---- & --- & ---- & --- & $\begin{array}{l}22.2 \\
20.5\end{array}$ & $\begin{array}{l}16.5 \\
1.5\end{array}$ & \\
\hline 31 & & -- & -- & --- & --- & --- & --- & --- & --- & 23.3 & 16.8 & 20.3 \\
\hline MC & 19.8 & 10.5 & 14.7 & & & & & & & 29.5 & 15.7 & \\
\hline
\end{tabular}

\begin{tabular}{|c|c|c|c|c|c|c|c|c|c|c|c|c|}
\hline \multirow[t]{2}{*}{ DAY } & MAX & MIN & MEAN & MAX & MIN & MEAN & MAX & MIN & MEAN & MAX & MIN & MEAN \\
\hline & \multicolumn{3}{|c|}{ SEPTEMBER } & \multicolumn{3}{|c|}{ OCTOBER } & \multicolumn{3}{|c|}{ NOVEMBER } & \multicolumn{3}{|c|}{ DECEMBER } \\
\hline $\begin{array}{l}1 \\
2 \\
3 \\
4 \\
5\end{array}$ & $\begin{array}{l}25.8 \\
24.9 \\
20.5 \\
20.2 \\
20.8\end{array}$ & $\begin{array}{l}18.3 \\
18.6 \\
17.7 \\
18.0 \\
14.4\end{array}$ & $\begin{array}{l}22.5 \\
21.6 \\
19.4 \\
19.4 \\
17.9\end{array}$ & $\begin{array}{l}16.5 \\
18.6 \\
19.2 \\
17.7 \\
16.0\end{array}$ & $\begin{array}{l}12.0 \\
14.4 \\
14.2 \\
13.7 \\
11.8\end{array}$ & $\begin{array}{l}14.1 \\
16.3 \\
16.5 \\
15.5 \\
14.3\end{array}$ & $\begin{array}{l}6.9 \\
8.1 \\
8.5 \\
7.8 \\
6.9\end{array}$ & $\begin{array}{l}6.0 \\
6.5 \\
6.9 \\
6.2 \\
6.2\end{array}$ & $\begin{array}{l}6.5 \\
7.4 \\
7.9 \\
6.9 \\
6.5\end{array}$ & $\begin{array}{l}3.0 \\
2.0 \\
0.9 \\
1.2 \\
0.9\end{array}$ & $\begin{array}{l}2.0 \\
0.2 \\
0.1 \\
0.2 \\
0.2\end{array}$ & $\begin{array}{l}2.5 \\
0.8 \\
0.4 \\
0.6 \\
0.6\end{array}$ \\
\hline $\begin{array}{r}6 \\
7 \\
8 \\
9 \\
10\end{array}$ & $\begin{array}{l}21.2 \\
21.2 \\
23.7 \\
25.8 \\
25.3\end{array}$ & $\begin{array}{l}12.3 \\
14.2 \\
15.7 \\
17.7 \\
16.0\end{array}$ & $\begin{array}{l}16.6 \\
17.6 \\
19.8 \\
21.5 \\
20.3\end{array}$ & $\begin{array}{l}14.4 \\
14.2 \\
15.4 \\
13.4 \\
11.6\end{array}$ & $\begin{array}{r}11.2 \\
10.7 \\
9.7 \\
10.1 \\
6.0\end{array}$ & $\begin{array}{r}12.3 \\
12.7 \\
12.5 \\
11.4 \\
7.7\end{array}$ & $\begin{array}{r}7.0 \\
8.3 \\
9.5 \\
10.5 \\
8.9\end{array}$ & $\begin{array}{l}6.3 \\
6.7 \\
8.1 \\
8.7 \\
8.0\end{array}$ & $\begin{array}{l}6.7 \\
7.6 \\
8.7 \\
9.4 \\
8.4\end{array}$ & $\begin{array}{l}1.2 \\
0.6 \\
0.7 \\
0.9 \\
0.9\end{array}$ & $\begin{array}{r}0.2 \\
0.3 \\
0.2 \\
-0.5 \\
-0.2\end{array}$ & $\begin{array}{l}0.6 \\
0.4 \\
0.4 \\
0.4 \\
0.3\end{array}$ \\
\hline $\begin{array}{l}11 \\
12 \\
13 \\
14 \\
15\end{array}$ & $\begin{array}{l}23.7 \\
23.3 \\
23.7 \\
22.2 \\
17.7\end{array}$ & $\begin{array}{l}15.4 \\
18.9 \\
18.6 \\
15.4 \\
15.2\end{array}$ & $\begin{array}{l}19.7 \\
21.1 \\
20.9 \\
18.8 \\
16.5\end{array}$ & $\begin{array}{r}8.9 \\
12.7 \\
15.2 \\
15.7 \\
14.7\end{array}$ & $\begin{array}{r}5.3 \\
4.8 \\
7.4 \\
10.1 \\
11.4\end{array}$ & $\begin{array}{r}6.7 \\
8.3 \\
11.2 \\
12.9 \\
13.8\end{array}$ & $\begin{array}{l}9.5 \\
9.7 \\
9.1 \\
9.1 \\
9.1\end{array}$ & $\begin{array}{l}7.6 \\
8.5 \\
8.1 \\
8.3 \\
6.9\end{array}$ & $\begin{array}{l}8.7 \\
9.0 \\
8.6 \\
8.7 \\
8.4\end{array}$ & $\begin{array}{l}0.2 \\
1.3 \\
0.2 \\
0.1 \\
0.5\end{array}$ & $\begin{array}{l}-0.7 \\
-0.7 \\
-0.6 \\
-0.7 \\
-0.4\end{array}$ & $\begin{array}{r}-0.2 \\
0.0 \\
-0.2 \\
-0.3 \\
0.0\end{array}$ \\
\hline $\begin{array}{l}16 \\
17 \\
18 \\
19 \\
20\end{array}$ & $\begin{array}{l}19.5 \\
18.0 \\
20.5 \\
19.8 \\
19.2\end{array}$ & $\begin{array}{l}15.2 \\
15.2 \\
15.2 \\
15.7 \\
17.7\end{array}$ & $\begin{array}{l}17.3 \\
16.6 \\
17.6 \\
17.9 \\
18.3\end{array}$ & $\begin{array}{l}12.5 \\
12.5 \\
11.8 \\
12.0 \\
12.7\end{array}$ & $\begin{array}{r}7.9 \\
8.9 \\
9.1 \\
10.5 \\
8.3\end{array}$ & $\begin{array}{l}10.7 \\
10.8 \\
10.6 \\
11.5 \\
10.7\end{array}$ & $\begin{array}{l}6.9 \\
8.0 \\
6.2 \\
3.9 \\
3.6\end{array}$ & $\begin{array}{l}5.2 \\
5.5 \\
3.1 \\
2.1 \\
1.8\end{array}$ & $\begin{array}{l}6.0 \\
6.4 \\
4.5 \\
2.9 \\
2.3\end{array}$ & $\begin{array}{l}0.3 \\
1.3 \\
1.6 \\
0.5 \\
0.3\end{array}$ & $\begin{array}{r}-0.1 \\
0.0 \\
0.2 \\
-0.1 \\
-0.2\end{array}$ & $\begin{array}{l}0.0 \\
0.4 \\
1.2 \\
0.2 \\
0.1\end{array}$ \\
\hline $\begin{array}{l}21 \\
22 \\
23 \\
24 \\
25\end{array}$ & $\begin{array}{l}21.2 \\
20.2 \\
17.7 \\
17.1 \\
18.9\end{array}$ & $\begin{array}{l}18.0 \\
16.0 \\
14.2 \\
14.7 \\
12.5\end{array}$ & $\begin{array}{l}19.5 \\
18.3 \\
16.2 \\
16.4 \\
15.2\end{array}$ & $\begin{array}{l}15.2 \\
14.4 \\
11.6 \\
12.5 \\
13.4\end{array}$ & $\begin{array}{l}9.1 \\
8.7 \\
6.3 \\
5.5 \\
7.0\end{array}$ & $\begin{array}{r}12.2 \\
10.8 \\
8.7 \\
9.1 \\
11.0\end{array}$ & $\begin{array}{l}2.7 \\
2.3 \\
1.6 \\
1.6 \\
1.6\end{array}$ & $\begin{array}{l}2.0 \\
1.3 \\
0.6 \\
0.3 \\
0.5\end{array}$ & $\begin{array}{l}2.2 \\
1.8 \\
1.1 \\
0.9 \\
0.8\end{array}$ & $\begin{array}{l}0.3 \\
0.1 \\
0.1 \\
0.2 \\
0.1\end{array}$ & $\begin{array}{l}-0.1 \\
-0.1 \\
-0.1 \\
-0.1 \\
-0.1\end{array}$ & $\begin{array}{l}0.1 \\
0.1 \\
0.1 \\
0.1 \\
0.1\end{array}$ \\
\hline $\begin{array}{l}26 \\
27 \\
28 \\
29 \\
30 \\
31\end{array}$ & $\begin{array}{r}15.2 \\
16.0 \\
13.7 \\
13.2 \\
14.2 \\
---\end{array}$ & $\begin{array}{r}12.3 \\
12.5 \\
10.7 \\
8.7 \\
9.7 \\
---\end{array}$ & $\begin{array}{r}14.0 \\
14.0 \\
12.8 \\
11.1 \\
12.1 \\
---\end{array}$ & $\begin{array}{r}14.2 \\
13.0 \\
11.8 \\
7.9 \\
6.3 \\
6.7\end{array}$ & $\begin{array}{r}10.5 \\
11.4 \\
7.4 \\
3.9 \\
3.4 \\
6.0\end{array}$ & $\begin{array}{r}12.1 \\
12.2 \\
10.4 \\
5.4 \\
4.9 \\
6.2\end{array}$ & $\begin{array}{l}1.6 \\
2.1 \\
3.1 \\
3.6 \\
3.4 \\
---\end{array}$ & $\begin{array}{l}0.5 \\
1.0 \\
2.0 \\
2.8 \\
2.8 \\
---\end{array}$ & $\begin{array}{l}0.9 \\
1.7 \\
2.6 \\
3.2 \\
3.1 \\
---\end{array}$ & $\begin{array}{l}0.1 \\
0.3 \\
0.1 \\
0.1 \\
0.1 \\
0.1\end{array}$ & $\begin{array}{r}-0.1 \\
-0.1 \\
-0.4 \\
-0.1 \\
0.1 \\
-0.4\end{array}$ & $\begin{array}{l}.1 \\
.1 \\
.0\end{array}$ \\
\hline MONTH & 25.8 & 8.7 & 17.7 & 19.2 & 3.4 & 11.1 & 10.5 & 0.3 & 5.3 & 3.0 & -0.7 & 0.3 \\
\hline
\end{tabular}


Table A15. Specific conductance and water temperature data collected May-November 2000 at station 101 (USGS identifier 01022820) Northeast Creek at Route 3 bridge near Bar Harbor, ME - Continued.

\begin{tabular}{|c|c|c|c|c|c|c|c|c|c|c|c|c|}
\hline \multirow[b]{2}{*}{ DAY } & \multicolumn{12}{|c|}{ DEEP } \\
\hline & MAX & MIN & MEAN & MAX & MIN & MEAN & MAX & MIN & MEAN & MAX & MIN & MEAN \\
\hline & \multicolumn{3}{|c|}{ MAY } & \multicolumn{3}{|c|}{ JUNE } & \multicolumn{3}{|c|}{ JULY } & \multicolumn{3}{|c|}{ AUGUST } \\
\hline 1 & --- & --- & --- & --- & --- & --- & 23.7 & 14.9 & 18.0 & --- & --- & --- \\
\hline 2 & --- & --- & --- & --- & --- & --- & 24.9 & 16.5 & 20.1 & --- & --- & --- \\
\hline 3 & --- & --- & --- & --- & --- & --- & 22.6 & 16.8 & 19.4 & --- & --- & --- \\
\hline 4 & --- & --- & --- & 19.2 & 13.9 & 16.5 & 23.0 & 15.7 & 19.6 & --- & --- & --- \\
\hline 5 & --- & --- & --- & 19.8 & 12.5 & 16.1 & 23.0 & 16.5 & 20.5 & --- & --- & --- \\
\hline 6 & --- & --- & --- & 20.5 & 12.7 & 16.1 & 24.5 & 16.8 & 21.2 & --- & --- & --- \\
\hline 7 & --- & --- & --- & 17.7 & 11.4 & 13.2 & 25.8 & 19.5 & 22.8 & --- & --- & --- \\
\hline 8 & --- & --- & --- & 18.3 & 12.5 & 15.4 & 25.3 & 21.2 & 22.7 & 26.2 & 23.3 & 25.0 \\
\hline 9 & --- & --- & --- & 22.2 & 15.5 & 18.5 & 24.9 & 21.5 & 23.0 & 27.1 & 24.5 & 26.1 \\
\hline 10 & --- & --- & --- & 20.5 & 18.0 & 19.0 & --- & --- & --- & 27.1 & 24.5 & 26.0 \\
\hline 11 & --- & --- & --- & 18.6 & 14.9 & 16.2 & --- & --- & --- & 29.5 & 25.3 & 27.0 \\
\hline 12 & --- & --- & --- & 19.8 & 14.4 & 16.6 & --- & --- & --- & 27.1 & 21.2 & 25.9 \\
\hline 13 & --- & --- & --- & 19.5 & 17.7 & 18.3 & --- & --- & --- & 23.7 & 19.8 & 22.4 \\
\hline 14 & --- & --- & --- & 20.2 & 17.9 & 18.7 & --- & --- & --- & 21.5 & 18.6 & 20.5 \\
\hline 15 & --- & --- & --- & 18.6 & 15.2 & 18.2 & --- & --- & --- & 21.2 & 18.3 & 19.2 \\
\hline 16 & --- & --- & --- & 19.8 & 15.2 & 16.1 & --- & --- & --- & 20.8 & 18.6 & 19.5 \\
\hline 17 & 17.7 & 14.2 & 15.6 & 21.2 & 18.9 & 19.6 & --- & --- & --- & 21.5 & 18.6 & 20.1 \\
\hline 18 & 14.9 & 13.7 & 14.5 & 21.9 & 20.8 & 21.4 & --- & --- & --- & 21.9 & 17.7 & 19.8 \\
\hline 19 & 14.9 & 12.5 & 13.6 & 22.6 & 19.5 & 20.6 & --- & --- & --- & 22.6 & 18.6 & 20.4 \\
\hline 20 & 17.4 & 11.8 & 14.6 & 22.2 & 20.1 & 21.0 & --- & --- & --- & 21.9 & 18.6 & 20.4 \\
\hline 21 & 17.1 & 14.9 & 15.8 & 21.9 & 20.1 & 20.8 & --- & --- & --- & 24.1 & 20.5 & 22.2 \\
\hline 22 & 18.6 & 14.7 & 16.5 & 21.2 & 18.0 & 19.3 & --- & --- & --- & 25.8 & 22.2 & 23.7 \\
\hline 23 & 17.1 & 14.2 & 15.4 & 21.5 & 19.8 & 20.6 & --- & --- & --- & 25.3 & 23.0 & 24.0 \\
\hline 24 & 14.2 & 11.6 & 13.0 & 22.2 & 21.2 & 21.8 & --- & --- & --- & 25.8 & 23.0 & 23.8 \\
\hline 25 & 11.6 & 11.2 & 11.4 & 23.0 & 21.9 & 22.5 & --- & --- & --- & 25.8 & 21.5 & 23.6 \\
\hline 26 & 16.6 & 10.5 & 13.3 & 25.3 & 22.6 & 23.5 & --- & --- & --- & 24.5 & 20.2 & 22.1 \\
\hline 27 & 17.1 & 14.2 & 15.8 & 26.6 & 23.0 & 25.3 & --- & --- & --- & 23.0 & 18.0 & 20.0 \\
\hline 28 & 16.5 & 14.4 & 15.2 & 25.8 & 22.2 & 23.3 & --- & --- & --- & 22.2 & 17.1 & 19.4 \\
\hline 29 & 15.2 & 13.9 & 14.5 & 23.3 & 17.1 & 21.3 & --- & --- & --- & 21.9 & 16.5 & 18.6 \\
\hline 30 & 19.2 & 12.9 & 15.9 & 19.2 & 14.9 & 17.5 & --- & --- & --- & 20.5 & 16.5 & 18.1 \\
\hline 31 & --- & --- & --- & --- & --- & --- & --- & --- & --- & 23.0 & 16.8 & 20.0 \\
\hline MONTH & 19.2 & 10.5 & 14.7 & 26.6 & 11.4 & 19.2 & & & & 29.5 & 16.5 & 22.0 \\
\hline \multirow[t]{2}{*}{ DAY } & MAX & MIN & MEAN & MAX & MIN & MEAN & MAX & MIN & MEAN & MAX & MIN & MEAN \\
\hline & & EPTEME & & & CTOBER & & & VEMBER & & & ECEMBE & \\
\hline 1 & 25.3 & 18.0 & 21.8 & 16.3 & 12.3 & 14.5 & 6.9 & 6.0 & 6.6 & 3.0 & 2.0 & 2.5 \\
\hline 2 & 24.5 & 18.6 & 21.0 & 17.7 & 15.7 & 16.7 & 8.1 & 6.5 & 7.4 & 2.0 & 0.2 & 0.8 \\
\hline 3 & 19.8 & 17.4 & 18.7 & 18.6 & 17.4 & 18.0 & 8.5 & 6.9 & 7.8 & 0.9 & 0.1 & 0.4 \\
\hline 4 & 19.8 & 19.2 & 19.4 & 18.6 & 17.7 & 18.3 & 7.9 & 6.2 & 6.9 & 1.2 & 0.2 & 0.6 \\
\hline 5 & 21.5 & 19.2 & 20.3 & 18.0 & 16.5 & 17.2 & 6.7 & 6.2 & 6.4 & 0.9 & 0.2 & 0.6 \\
\hline 6 & 22.2 & 19.2 & 20.9 & 17.4 & 15.4 & 16.6 & 7.0 & 6.3 & 6.7 & 1.2 & 0.2 & 0.7 \\
\hline 7 & 22.6 & 20.5 & 21.7 & 17.4 & 16.8 & 17.2 & 8.3 & 6.9 & 7.6 & 0.6 & 0.3 & 0.4 \\
\hline 8 & 24.1 & 21.9 & 23.0 & 18.0 & 16.8 & 17.4 & 9.5 & 8.1 & 8.7 & 0.9 & 0.2 & 0.4 \\
\hline 9 & 25.8 & 22.9 & 24.2 & 17.9 & 13.2 & 17.2 & 9.3 & 8.7 & 9.0 & 0.3 & -0.6 & -0.2 \\
\hline 10 & 25.3 & 22.2 & 23.7 & 14.2 & 13.4 & 13.8 & 9.3 & 8.1 & 8.7 & 0.2 & -0.6 & -0.2 \\
\hline 11 & 23.7 & 20.8 & 22.4 & 14.4 & 13.9 & 14.2 & 9.3 & 8.1 & 8.7 & 0.2 & -0.7 & -0.3 \\
\hline 12 & 23.3 & 18.6 & 22.4 & 14.2 & 11.8 & 13.9 & 9.5 & 8.5 & 9.3 & 1.3 & -0.7 & 0.0 \\
\hline 13 & 21.2 & 17.7 & 19.7 & 12.5 & 11.4 & 12.1 & 9.1 & 8.5 & 8.8 & 0.1 & -0.6 & -0.2 \\
\hline 14 & 19.5 & 17.1 & 18.4 & 13.2 & 11.6 & 12.4 & 9.1 & 8.7 & 8.9 & 0.1 & -0.7 & -0.2 \\
\hline 15 & 17.1 & 15.5 & 16.0 & 14.7 & 12.7 & 13.7 & 9.1 & 8.1 & 8.6 & 0.5 & -0.6 & 0.0 \\
\hline 16 & 18.9 & 15.7 & 17.3 & 14.2 & 10.5 & 12.0 & 8.3 & 5.7 & 6.8 & 0.2 & -0.1 & 0.1 \\
\hline 17 & 17.7 & 15.2 & 16.7 & 12.0 & 9.9 & 11.3 & 8.0 & 5.5 & 6.7 & 1.6 & 0.0 & 0.5 \\
\hline 18 & 20.5 & 16.8 & 18.6 & 12.3 & 10.1 & 11.4 & 7.8 & 3.3 & 5.4 & 1.6 & 0.2 & 1.2 \\
\hline 19 & 20.2 & 18.6 & 19.2 & 12.0 & 10.5 & 11.1 & 4.2 & 2.2 & 3.2 & 0.5 & -0.2 & 0.2 \\
\hline 20 & 19.2 & 17.7 & 18.6 & 13.2 & 11.4 & 12.1 & 4.0 & 2.4 & 3.2 & 0.3 & -0.2 & 0.1 \\
\hline 21 & 20.5 & 17.7 & 18.9 & 14.9 & 12.5 & 13.4 & 3.7 & 2.7 & 3.2 & 0.3 & -0.1 & 0.1 \\
\hline 22 & 21.2 & 18.0 & 19.5 & 15.2 & 14.4 & 14.7 & 2.7 & 1.7 & 2.4 & 0.1 & -0.1 & 0.1 \\
\hline 23 & 18.9 & 16.3 & 17.8 & 14.4 & 10.5 & 13.4 & 2.3 & 1.1 & 1.6 & 0.1 & -0.1 & 0.1 \\
\hline 24 & 17.1 & 14.9 & 16.4 & 11.2 & 8.3 & 10.1 & 1.5 & 0.6 & 1.1 & 0.2 & -0.1 & 0.1 \\
\hline 25 & 16.0 & 12.5 & 14.7 & 11.4 & 8.9 & 10.5 & 1.5 & 0.3 & 1.1 & 0.1 & -0.1 & 0.1 \\
\hline 26 & 15.2 & 12.9 & 14.0 & 13.2 & 10.5 & 11.4 & 1.7 & 0.9 & 1.5 & 0.1 & -0.1 & 0.1 \\
\hline 27 & 15.7 & 12.5 & 14.0 & 12.5 & 11.2 & 11.7 & 2.1 & 1.7 & 1.9 & 0.3 & -0.1 & 0.1 \\
\hline 28 & 13.7 & 11.4 & 12.8 & 11.8 & 8.1 & 10.5 & 3.1 & 2.0 & 2.6 & 0.1 & -0.6 & -0.1 \\
\hline 29 & 13.2 & 9.3 & 11.3 & 8.5 & 4.8 & 5.8 & 3.6 & 2.8 & 3.2 & 0.2 & 0.1 & 0.1 \\
\hline 30 & 14.2 & 10.5 & 12.4 & 6.5 & 4.5 & 5.7 & 3.4 & 2.8 & 3.1 & 0.2 & 0.0 & 0.1 \\
\hline 31 & --- & --- & --- & 6.9 & 6.3 & 6.6 & --- & --- & --- & 0.1 & -0.4 & 0.1 \\
\hline MONTH & 25.8 & 9.3 & 18.5 & 18.6 & 4.5 & 13.1 & 9.5 & 0.3 & 5.6 & 3.0 & -0.7 & 0.3 \\
\hline
\end{tabular}


Table A16. Specific conductance and water temperature data collected May-November 2000 at station 102 (USGS identifier 442517068190501) Northeast Creek near Bar Harbor, ME [Data are presented as minimum, mean and maximum daily values of continuous data at 15-minute intervals. Shallow and deep data were collected at fixed depths located, respectively, about $0.2 \mathrm{~m}$ below the water surface and about $0.2 \mathrm{~m}$ above the stream bottom.]

SHALLOW

Specific conductance, water, microsiemens per centimeter at 25 degrees Celsius

\begin{tabular}{|c|c|c|c|c|c|c|c|c|c|c|c|c|}
\hline \multirow[t]{2}{*}{ DAY } & MAX & MIN & MEAN & MAX & MIN & MEAN & MAX & MIN & MEAN & MAX & MIN & MEAN \\
\hline & & MAY & & \multicolumn{3}{|c|}{ JUNE } & \multicolumn{3}{|c|}{ JULY } & \multicolumn{3}{|c|}{ AUGUST } \\
\hline 1 & --- & --- & --- & 7000 & 295 & 797 & --- & --- & --- & --- & --- & \\
\hline 2 & --- & --- & --- & 7000 & 1510 & 6280 & --- & --- & --- & --- & --- & \\
\hline 3 & --- & --- & --- & 7000 & 5210 & 6910 & --- & --- & --- & --- & --- & \\
\hline 4 & --- & --- & --- & 7000 & 7000 & 7000 & --- & --- & --- & --- & --- & \\
\hline 5 & --- & --- & --- & 7000 & 6500 & 6990 & --- & --- & --- & --- & --- & - \\
\hline 8 & --- & --- & --- & 7000 & 2120 & 3310 & 11900 & 6590 & 8220 & 21900 & 10200 & 15900 \\
\hline 9 & --- & --- & --- & 2120 & 1630 & 1830 & 8460 & 5710 & 7150 & 11400 & 9240 & 10000 \\
\hline 10 & --- & --- & --- & 1640 & 1040 & 1220 & --- & --- & -- & 22000 & 9620 & 13500 \\
\hline 11 & --- & --- & --- & 1180 & 1040 & 1130 & --- & --- & --- & 22400 & 21300 & 21900 \\
\hline 12 & --- & --- & --- & 1150 & 901 & 1020 & --- & --- & --- & 22400 & 21000 & 21700 \\
\hline 13 & --- & --- & --- & 1070 & 846 & 988 & --- & --- & --- & 24000 & 20800 & 21500 \\
\hline 14 & --- & --- & --- & 1310 & 1020 & 1110 & --- & --- & --- & 26300 & 21300 & 22400 \\
\hline 15 & --- & --- & --- & 2100 & 1150 & 1250 & --- & --- & --- & 25900 & 21800 & 23100 \\
\hline 16 & --- & --- & --- & 2350 & 1240 & 1420 & --- & --- & --- & 27600 & 22500 & 24200 \\
\hline 17 & --- & --- & --- & 3920 & 1380 & 1760 & --- & --- & --- & 28900 & 23300 & 25600 \\
\hline 18 & 304 & 216 & 245 & 6540 & 1170 & 1570 & --- & --- & --- & 26400 & 24000 & 25000 \\
\hline 19 & 308 & 132 & 199 & 1420 & 958 & 1180 & --- & --- & --- & 37800 & 23600 & 24600 \\
\hline 20 & 167 & 128 & 143 & 1200 & 1030 & 1130 & --- & --- & --- & 28800 & 22200 & 24000 \\
\hline 21 & 216 & 164 & 180 & 1190 & 1060 & 1130 & --- & --- & --- & --- & --- & \\
\hline 22 & 299 & 216 & 247 & 1310 & 1110 & 1220 & --- & --- & --- & --- & --- & \\
\hline 23 & 335 & 298 & 313 & 1320 & 1220 & 1270 & --- & --- & --- & --- & --- & \\
\hline 24 & 314 & 226 & 255 & 1260 & 1020 & 1160 & --- & --- & --- & --- & --- & \\
\hline 25 & 243 & 69 & 114 & 1140 & 1060 & 1100 & --- & --- & --- & --- & --- & \\
\hline 26 & 82 & 68 & 73 & 1230 & 1060 & 1140 & --- & --- & --- & --- & --- & \\
\hline 27 & 107 & 82 & 94 & 3600 & 1100 & 1490 & --- & --- & --- & --- & --- & \\
\hline 28 & 121 & 103 & 111 & --- & --- & --- & --- & --- & --- & --- & --- & \\
\hline 29 & 152 & 119 & 132 & --- & --- & --- & --- & --- & --- & 46000 & 39600 & 42300 \\
\hline 30 & 222 & 152 & 183 & --- & --- & --- & --- & --- & --- & 46100 & 41100 & 43400 \\
\hline 31 & 347 & 222 & 273 & --- & --- & --- & --- & --- & --- & 45700 & 38300 & 43100 \\
\hline MONTH & 347 & 68 & 183 & 7000 & 295 & 2560 & & & & 46100 & 9240 & 25100 \\
\hline
\end{tabular}

\begin{tabular}{|c|c|c|c|c|c|c|c|c|c|}
\hline DAY & MAX & MIN & MEAN & MAX & MIN & MEAN & MAX & MIN & MEAN \\
\hline & \multicolumn{3}{|c|}{ SEPTEMBER } & \multicolumn{3}{|c|}{ OCTOBER } & \multicolumn{3}{|c|}{ NOVEMBER } \\
\hline 1 & 44700 & 40400 & 42500 & 42100 & 31800 & 34100 & 2010 & 642 & 1170 \\
\hline 2 & 42700 & 38300 & 40700 & 36200 & 30900 & 32500 & 727 & 397 & 488 \\
\hline 3 & 40600 & 34100 & 37700 & 37100 & 30100 & 32000 & 2080 & 396 & 434 \\
\hline 4 & 36100 & 29100 & 32800 & 32800 & 29900 & 31200 & 657 & 440 & 476 \\
\hline 5 & 34500 & 26200 & 29200 & --- & --- & --- & 637 & 517 & 573 \\
\hline 6 & 26300 & 24600 & 25500 & --- & --- & --- & 646 & 598 & 625 \\
\hline 7 & 25600 & 24200 & 24800 & --- & --- & --- & 832 & 568 & 589 \\
\hline 8 & 25600 & 24100 & 24800 & 16400 & 11900 & 13900 & 1480 & 566 & 602 \\
\hline 9 & 29400 & 24400 & 25400 & 17800 & 12000 & 14500 & 3160 & 617 & 786 \\
\hline 10 & 28600 & 26300 & 27400 & 16000 & 6970 & 9290 & 1510 & 394 & 661 \\
\hline 11 & 29000 & 26100 & 27500 & --- & --- & --- & --- & --- & --- \\
\hline 12 & 29400 & 26600 & 27200 & --- & --- & --- & --- & --- & --- \\
\hline 13 & 30900 & 28100 & 29200 & --- & --- & --- & --- & --- & --- \\
\hline 14 & 40100 & 29200 & 30300 & --- & --- & --- & --- & --- & --- \\
\hline 15 & 37700 & 29000 & 32700 & --- & --- & --- & --- & --- & --- \\
\hline 16 & --- & --- & --- & --- & --- & --- & 5860 & 655 & 1810 \\
\hline 17 & 37000 & 30900 & 32900 & --- & --- & --- & --- & --- & --- \\
\hline 18 & 32000 & 29600 & 30500 & --- & --- & --- & 674 & 451 & 553 \\
\hline 19 & 48200 & 27900 & 29500 & --- & --- & --- & 473 & 439 & 456 \\
\hline 20 & 31800 & 27500 & 29900 & --- & --- & --- & 550 & 470 & 492 \\
\hline 21 & 34400 & 26600 & 29600 & --- & --- & --- & 577 & 466 & 539 \\
\hline 22 & 35600 & 30200 & 31400 & --- & --- & --- & 530 & 345 & 412 \\
\hline 23 & --- & --- & --- & --- & --- & --- & 400 & 323 & 356 \\
\hline 24 & --- & --- & --- & --- & --- & --- & 396 & 323 & 361 \\
\hline 25 & 42000 & 29800 & 32700 & --- & --- & --- & 459 & 368 & 418 \\
\hline 26 & 44600 & 32600 & 38000 & --- & --- & --- & 733 & 434 & 495 \\
\hline 27 & 45600 & 35900 & 40800 & --- & --- & --- & 514 & 102 & 247 \\
\hline 28 & 44800 & 36800 & 41200 & --- & --- & --- & --- & --- & --- \\
\hline 29 & 42800 & 36000 & 39000 & --- & --- & --- & --- & --- & --- \\
\hline 30 & 39400 & 32900 & 36100 & --- & --- & --- & --- & --- & --- \\
\hline 31 & 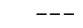 & --- & --- & --- & --- & --- & --- & --- & --- \\
\hline MONTH & 48200 & 24100 & 32200 & 42100 & 6970 & 23900 & 5860 & 102 & 597 \\
\hline
\end{tabular}


Table A16. Specific conductance and water temperature data collected May-November 2000 at station 102 (USGS identifier 442517068190501) Northeast Creek near Bar Harbor, ME - Continued.

\section{DEEP}

Specific conductance, water, microsiemens per centimeter at 25 degrees Celsius

\begin{tabular}{|c|c|c|c|c|c|c|c|c|c|c|c|c|}
\hline \multirow[t]{2}{*}{ DAY } & MAX & MIN & MEAN & MAX & MIN & MEAN & MAX & MIN & MEAN & MAX & MIN & MEAN \\
\hline & & MAY & & \multicolumn{3}{|c|}{ JUNE } & \multicolumn{3}{|c|}{ JULY } & \multicolumn{3}{|c|}{ AUGUST } \\
\hline 1 & --- & --- & --- & --- & --- & --- & --- & --- & --- & --- & --- & --- \\
\hline 2 & --- & --- & --- & --- & --- & --- & --- & --- & --- & --- & --- & --- \\
\hline 3 & --- & --- & --- & --- & --- & --- & --- & --- & --- & --- & --- & --- \\
\hline 4 & --- & --- & --- & --- & --- & --- & --- & --- & --- & --- & --- & --- \\
\hline 5 & --- & --- & --- & --- & --- & --- & --- & --- & --- & --- & --- & --- \\
\hline 6 & --- & --- & --- & --- & --- & --- & --- & --- & --- & --- & --- & \\
\hline 7 & --- & --- & --- & --- & --- & --- & 28000 & 20000 & 23400 & --- & --- & \\
\hline 8 & --- & --- & --- & --- & --- & --- & 20600 & 16200 & 19100 & 27500 & 21600 & 26100 \\
\hline 9 & --- & --- & --- & --- & --- & --- & 18400 & 11900 & 16400 & 27900 & 16500 & 25600 \\
\hline 10 & --- & --- & --- & --- & --- & --- & --- & --- & --- & --- & --- & \\
\hline 11 & --- & --- & --- & --- & --- & --- & --- & --- & --- & 25800 & 23300 & 24400 \\
\hline 12 & --- & --- & --- & --- & --- & --- & --- & --- & --- & 26500 & 23700 & 25100 \\
\hline 13 & --- & --- & --- & --- & --- & --- & --- & --- & --- & 25800 & 24100 & 25000 \\
\hline 14 & --- & --- & --- & --- & --- & --- & --- & --- & --- & 26400 & 15300 & 24900 \\
\hline 15 & --- & --- & --- & --- & --- & --- & --- & --- & --- & 27200 & 16300 & 25700 \\
\hline 16 & --- & --- & --- & --- & --- & --- & --- & --- & --- & 28700 & 25000 & 27300 \\
\hline 17 & --- & --- & --- & --- & --- & --- & --- & --- & --- & 30500 & 27000 & 200 \\
\hline 18 & --- & --- & --- & --- & --- & --- & --- & --- & --- & 29400 & 25400 & 27100 \\
\hline 19 & --- & --- & --- & --- & --- & --- & --- & --- & --- & 28600 & 26400 & 27600 \\
\hline 20 & --- & --- & --- & --- & --- & --- & --- & --- & --- & 28500 & 25600 & 27300 \\
\hline 21 & --- & --- & --- & --- & --- & --- & --- & --- & --- & --- & --- & - \\
\hline 22 & --- & --- & --- & --- & --- & --- & --- & --- & --- & --- & --- & \\
\hline 23 & --- & --- & --- & --- & --- & --- & --- & --- & --- & --- & --- & \\
\hline 24 & --- & --- & --- & --- & --- & --- & --- & --- & --- & --- & --- & \\
\hline 25 & --- & --- & --- & --- & --- & --- & --- & --- & --- & --- & --- & - \\
\hline 26 & --- & --- & --- & --- & --- & --- & --- & --- & --- & --- & --- & \\
\hline 27 & --- & --- & --- & --- & --- & --- & --- & --- & --- & --- & -- & \\
\hline 28 & --- & --- & --- & --- & --- & --- & --- & --- & --- & --- & -- & \\
\hline 29 & --- & --- & --- & --- & --- & --- & --- & --- & --- & 47300 & 32100 & 43400 \\
\hline 30 & --- & --- & --- & --- & --- & --- & --- & --- & --- & 47300 & 26100 & 42000 \\
\hline 31 & --- & --- & --- & --- & --- & --- & --- & --- & --- & 46000 & 23300 & 41800 \\
\hline MONTH & --- & --- & --- & --- & --- & --- & & & & 47300 & 15300 & 29500 \\
\hline
\end{tabular}

\begin{tabular}{|c|c|c|c|c|c|c|c|c|c|}
\hline \multirow[t]{2}{*}{ DAY } & MAX & MIN & MEAN & MAX & MIN & MEAN & MAX & MIN & MEAN \\
\hline & \multicolumn{3}{|c|}{ SEPTEMBER } & \multicolumn{3}{|c|}{ OCTOBER } & \multicolumn{3}{|c|}{ NOVEMBER } \\
\hline $\begin{array}{l}1 \\
2 \\
3 \\
4 \\
5\end{array}$ & $\begin{array}{l}45800 \\
43900 \\
42300 \\
41200 \\
41200\end{array}$ & $\begin{array}{l}21900 \\
30200 \\
23700 \\
23300 \\
18600\end{array}$ & $\begin{array}{l}41300 \\
40200 \\
35400 \\
36100 \\
34200\end{array}$ & $\begin{array}{l}38300 \\
37800 \\
37600 \\
36900 \\
36000\end{array}$ & $\begin{array}{l}35700 \\
35900 \\
35000 \\
33000 \\
34600\end{array}$ & $\begin{array}{l}37000 \\
37000 \\
36600 \\
35200 \\
35200\end{array}$ & $\begin{array}{r}2050 \\
659 \\
454 \\
532 \\
650\end{array}$ & $\begin{array}{l}657 \\
409 \\
406 \\
453 \\
532\end{array}$ & $\begin{array}{r}1190 \\
488 \\
421 \\
486 \\
588\end{array}$ \\
\hline $\begin{array}{r}6 \\
7 \\
8 \\
9 \\
10\end{array}$ & $\begin{array}{l}40800 \\
39600 \\
36600 \\
36200 \\
34100\end{array}$ & $\begin{array}{l}24700 \\
20100 \\
24600 \\
21100 \\
18100\end{array}$ & $\begin{array}{l}33800 \\
32600 \\
32400 \\
30900 \\
30100\end{array}$ & $\begin{array}{l}36200 \\
35700 \\
32200 \\
29600 \\
26200\end{array}$ & $\begin{array}{r}34900 \\
28900 \\
20200 \\
25600 \\
7300\end{array}$ & $\begin{array}{l}35700 \\
32200 \\
28000 \\
28300 \\
19300\end{array}$ & $\begin{array}{r}663 \\
614 \\
632 \\
719 \\
2400\end{array}$ & $\begin{array}{l}614 \\
584 \\
582 \\
632 \\
409\end{array}$ & $\begin{array}{l}642 \\
603 \\
603 \\
675 \\
716\end{array}$ \\
\hline $\begin{array}{l}11 \\
12 \\
13 \\
14 \\
15\end{array}$ & $\begin{array}{l}34200 \\
33500 \\
32300 \\
31600 \\
35500\end{array}$ & $\begin{array}{l}19300 \\
15300 \\
13900 \\
19500 \\
21500\end{array}$ & $\begin{array}{l}30400 \\
21700 \\
25500 \\
26900 \\
29300\end{array}$ & $\begin{array}{l}--- \\
--- \\
--- \\
--- \\
---\end{array}$ & $\begin{array}{l}--- \\
--- \\
--- \\
--- \\
---\end{array}$ & $\begin{array}{l}--- \\
--- \\
--- \\
---\end{array}$ & $\begin{array}{l}17900 \\
19300 \\
24400 \\
34500 \\
33900\end{array}$ & $\begin{array}{r}383 \\
681 \\
812 \\
6070 \\
3890\end{array}$ & $\begin{array}{r}8660 \\
10100 \\
13400 \\
26900 \\
19200\end{array}$ \\
\hline $\begin{array}{l}16 \\
17 \\
18 \\
19 \\
20\end{array}$ & $\begin{array}{l}39700 \\
36500 \\
36700 \\
35700 \\
34600\end{array}$ & $\begin{array}{l}24200 \\
23500 \\
16300 \\
17200 \\
20400\end{array}$ & $\begin{array}{l}28400 \\
32000 \\
32100 \\
32300 \\
29900\end{array}$ & $\begin{array}{l}--- \\
--- \\
--- \\
--- \\
---\end{array}$ & $\begin{array}{l}--- \\
--- \\
--- \\
--- \\
---\end{array}$ & $\begin{array}{l}--- \\
--- \\
--- \\
--- \\
---\end{array}$ & $\begin{array}{r}4640 \\
1230 \\
646 \\
489 \\
560\end{array}$ & $\begin{array}{l}682 \\
567 \\
470 \\
451 \\
485\end{array}$ & $\begin{array}{r}1500 \\
679 \\
566 \\
466 \\
504\end{array}$ \\
\hline $\begin{array}{l}21 \\
22 \\
23 \\
24 \\
25\end{array}$ & $\begin{array}{l}35200 \\
35400 \\
33500 \\
37200 \\
43700\end{array}$ & $\begin{array}{l}15900 \\
18800 \\
19200 \\
24200 \\
19800\end{array}$ & $\begin{array}{l}27800 \\
28300 \\
27300 \\
30500 \\
33300\end{array}$ & $\begin{array}{l}--- \\
--- \\
--- \\
--- \\
---\end{array}$ & $\begin{array}{l}--- \\
--- \\
--- \\
--- \\
---\end{array}$ & $\begin{array}{l}--- \\
--- \\
--- \\
--- \\
---\end{array}$ & $\begin{array}{l}617 \\
547 \\
416 \\
409 \\
474\end{array}$ & $\begin{array}{l}481 \\
355 \\
333 \\
333 \\
379\end{array}$ & $\begin{array}{l}566 \\
443 \\
380 \\
372 \\
431\end{array}$ \\
\hline $\begin{array}{l}26 \\
27 \\
28 \\
29 \\
30 \\
31\end{array}$ & $\begin{array}{r}46000 \\
46200 \\
45900 \\
43200 \\
41300 \\
----\end{array}$ & $\begin{array}{r}40800 \\
36400 \\
37100 \\
40300 \\
33800 \\
----\end{array}$ & $\begin{array}{r}42800 \\
43500 \\
43500 \\
41700 \\
38600 \\
---\end{array}$ & $\begin{array}{l}--- \\
--- \\
--- \\
--- \\
--- \\
---\end{array}$ & $\begin{array}{l}--- \\
--- \\
--- \\
--- \\
--- \\
---\end{array}$ & $\begin{array}{l}--- \\
--- \\
--- \\
--- \\
--- \\
---\end{array}$ & $\begin{array}{l}824 \\
534 \\
--- \\
--- \\
--- \\
---\end{array}$ & $\begin{array}{l}448 \\
105 \\
--- \\
--- \\
--- \\
---\end{array}$ & $\begin{array}{l}513 \\
256 \\
--- \\
--- \\
--- \\
---\end{array}$ \\
\hline MONTH & 46200 & 13900 & 33100 & 38300 & 7300 & 32400 & 34500 & 105 & 3380 \\
\hline
\end{tabular}


Table A16. Specific conductance and water temperature data collected May-November 2000 at station 102 (USGS identifier 442517068190501) Northeast Creek near Bar Harbor, ME - Continued.

\begin{tabular}{|c|c|c|c|c|c|c|c|c|c|c|c|c|}
\hline \multirow[b]{2}{*}{ DAY } & \multicolumn{12}{|c|}{ SHALLOW } \\
\hline & MAX & MIN & MEAN & MAX & MIN & MEAN & MAX & MIN & MEAN & MAX & MIN & MEAN \\
\hline & & MAY & & & JUNE & & & JULY & & & AUGUST & \\
\hline 1 & --- & --- & --- & 24.4 & 16.1 & 19.5 & --- & --- & --- & --- & --- & --- \\
\hline 2 & --- & --- & --- & 21.4 & 15.6 & 19.4 & --- & --- & --- & --- & --- & --- \\
\hline 3 & --- & --- & --- & 21.7 & 13.5 & 17.6 & --- & --- & --- & --- & --- & --- \\
\hline 4 & --- & --- & --- & 20.3 & 15.0 & 18.0 & --- & --- & --- & --- & --- & --- \\
\hline 5 & --- & --- & --- & 22.4 & 14.3 & 18.5 & --- & --- & --- & --- & --- & --- \\
\hline 6 & --- & --- & --- & 20.6 & 16.4 & 17.8 & --- & --- & --- & --- & --- & --- \\
\hline 7 & --- & --- & --- & 17.2 & 12.8 & 14.5 & 26.5 & 21.0 & 23.6 & --- & --- & --- \\
\hline 8 & --- & --- & --- & 19.0 & 13.5 & 16.3 & 23.2 & 19.3 & 21.2 & 28.8 & 20.7 & 23.9 \\
\hline 9 & --- & --- & --- & 23.2 & 15.3 & 18.9 & 26.0 & 17.5 & 21.5 & 26.0 & 18.4 & 22.4 \\
\hline 10 & --- & --- & --- & 20.6 & 17.8 & 18.5 & --- & --- & --- & 27.8 & 20.0 & 23.6 \\
\hline 11 & --- & --- & --- & 17.8 & 14.2 & 15.3 & --- & --- & --- & 28.3 & 20.3 & 23.9 \\
\hline 12 & --- & --- & --- & 22.1 & 13.3 & 17.1 & --- & --- & --- & 23.9 & 19.6 & 21.7 \\
\hline 13 & --- & --- & --- & 21.0 & 15.8 & 18.3 & --- & --- & --- & 24.4 & 20.3 & 21.8 \\
\hline 14 & --- & --- & --- & 22.4 & 16.1 & 19.0 & --- & --- & --- & 22.8 & 19.0 & 21.4 \\
\hline 15 & --- & --- & --- & 19.3 & 16.9 & 17.6 & --- & --- & --- & 25.2 & 20.7 & 22.7 \\
\hline 16 & --- & --- & --- & 26.5 & 16.1 & 19.9 & --- & --- & --- & 24.4 & 21.0 & 22.9 \\
\hline 17 & --- & --- & --- & 30.3 & 20.7 & 24.1 & --- & --- & --- & 25.6 & 20.0 & 23.0 \\
\hline 18 & 16.4 & 13.5 & 14.1 & 26.9 & 21.0 & 23.5 & --- & --- & --- & 24.8 & 18.1 & 21.4 \\
\hline 19 & 15.3 & 11.9 & 13.6 & 28.3 & 20.7 & 23.8 & --- & --- & --- & 24.8 & 19.3 & 22.1 \\
\hline 20 & 18.1 & 11.0 & 14.5 & 25.6 & 20.6 & 22.8 & --- & --- & --- & 23.2 & 18.1 & 20.5 \\
\hline 21 & 16.7 & 13.5 & 15.3 & 23.2 & 19.7 & 21.6 & --- & --- & --- & --- & --- & --- \\
\hline 22 & 18.4 & 14.0 & 16.1 & 28.3 & 18.4 & 21.9 & --- & --- & --- & --- & --- & --- \\
\hline 23 & 16.6 & 13.8 & 14.9 & 26.9 & 20.7 & 23.7 & --- & --- & --- & --- & --- & --- \\
\hline 24 & 14.2 & 11.4 & 12.6 & 28.8 & 21.0 & 24.3 & --- & --- & --- & --- & --- & --- \\
\hline 25 & 11.7 & 10.8 & 11.3 & 26.9 & 20.3 & 23.2 & --- & --- & --- & --- & --- & --- \\
\hline 26 & 17.2 & 10.4 & 13.6 & 32.6 & 21.7 & 25.9 & --- & --- & --- & --- & --- & --- \\
\hline 27 & 17.8 & 13.5 & 15.7 & 28.8 & 23.2 & 25.7 & --- & --- & --- & --- & --- & --- \\
\hline 28 & 16.1 & 13.5 & 14.9 & --- & --- & --- & --- & --- & --- & --- & --- & --- \\
\hline 29 & 15.3 & 13.0 & 14.1 & --- & --- & --- & --- & --- & --- & 24.4 & 18.4 & 20.9 \\
\hline 30 & 20.0 & 12.3 & 15.9 & --- & --- & --- & --- & --- & --- & 22.1 & 18.1 & 20.0 \\
\hline 31 & 20.0 & 14.3 & 17.1 & --- & --- & --- & --- & --- & --- & 25.6 & 19.4 & 22.2 \\
\hline MONTH & 20.0 & 10.4 & 14.6 & 32.6 & 12.8 & 20.2 & & & & 28.8 & 18.1 & 22.1 \\
\hline
\end{tabular}

\begin{tabular}{|c|c|c|c|c|c|c|c|c|c|}
\hline DAY & MAX & MIN & MEAN & MAX & MIN & MEAN & MAX & MIN & MEAN \\
\hline & \multicolumn{3}{|c|}{ SEPTEMBER } & \multicolumn{3}{|c|}{ OCTOBER } & \multicolumn{3}{|c|}{ NOVEMBER } \\
\hline $\begin{array}{l}1 \\
2 \\
3 \\
4 \\
5\end{array}$ & $\begin{array}{l}27.8 \\
26.0 \\
21.4 \\
20.3 \\
20.7\end{array}$ & $\begin{array}{l}22.1 \\
20.6 \\
19.3 \\
17.2 \\
13.0\end{array}$ & $\begin{array}{l}24.7 \\
23.0 \\
20.1 \\
19.0 \\
16.6\end{array}$ & $\begin{array}{l}17.8 \\
19.4 \\
21.0 \\
18.4 \\
18.4\end{array}$ & $\begin{array}{l}11.6 \\
14.5 \\
14.8 \\
14.0 \\
13.3\end{array}$ & $\begin{array}{l}14.4 \\
16.7 \\
17.5 \\
16.4 \\
15.4\end{array}$ & $\begin{array}{l}6.9 \\
8.4 \\
9.0 \\
7.8 \\
6.9\end{array}$ & $\begin{array}{l}5.8 \\
6.4 \\
6.5 \\
6.0 \\
5.8\end{array}$ & $\begin{array}{l}6.4 \\
7.4 \\
7.8 \\
6.5 \\
6.4\end{array}$ \\
\hline $\begin{array}{r}6 \\
7 \\
8 \\
9 \\
10\end{array}$ & $\begin{array}{l}21.0 \\
20.0 \\
21.7 \\
25.2 \\
24.8\end{array}$ & $\begin{array}{l}11.6 \\
12.8 \\
14.5 \\
16.1 \\
14.2\end{array}$ & $\begin{array}{l}16.1 \\
16.3 \\
18.3 \\
20.2 \\
18.6\end{array}$ & $\begin{array}{r}15.0 \\
14.3 \\
14.3 \\
11.4 \\
8.2\end{array}$ & $\begin{array}{r}11.7 \\
10.6 \\
9.3 \\
7.1 \\
5.0\end{array}$ & $\begin{array}{r}13.2 \\
12.5 \\
11.9 \\
9.5 \\
6.7\end{array}$ & $\begin{array}{r}7.1 \\
8.6 \\
9.3 \\
10.2 \\
8.9\end{array}$ & $\begin{array}{l}6.2 \\
6.7 \\
7.8 \\
8.4 \\
7.8\end{array}$ & $\begin{array}{l}6.6 \\
7.6 \\
8.6 \\
9.3 \\
8.1\end{array}$ \\
\hline $\begin{array}{l}11 \\
12 \\
13 \\
14 \\
15\end{array}$ & $\begin{array}{l}22.4 \\
22.4 \\
25.2 \\
24.0 \\
21.3\end{array}$ & $\begin{array}{l}11.6 \\
18.7 \\
20.3 \\
18.1 \\
18.1\end{array}$ & $\begin{array}{l}17.9 \\
20.6 \\
22.1 \\
21.1 \\
19.5\end{array}$ & $\begin{array}{l}--- \\
--- \\
--- \\
--- \\
---\end{array}$ & $\begin{array}{l}--- \\
--- \\
--- \\
--- \\
---\end{array}$ & $\begin{array}{l}--- \\
--- \\
--- \\
--- \\
---\end{array}$ & $\begin{array}{r}9.3 \\
10.0 \\
9.2 \\
8.9 \\
9.2\end{array}$ & $\begin{array}{l}7.6 \\
8.4 \\
8.0 \\
8.2 \\
6.5\end{array}$ & $\begin{array}{l}8.5 \\
9.1 \\
8.6 \\
8.7 \\
8.4\end{array}$ \\
\hline $\begin{array}{l}16 \\
17 \\
18 \\
19 \\
20\end{array}$ & $\begin{array}{l}21.0 \\
18.4 \\
22.1 \\
20.0 \\
20.7\end{array}$ & $\begin{array}{l}16.9 \\
15.0 \\
14.8 \\
15.8 \\
17.8\end{array}$ & $\begin{array}{l}18.9 \\
16.8 \\
18.2 \\
18.2 \\
19.1\end{array}$ & $\begin{array}{l}--- \\
--- \\
--- \\
--- \\
---\end{array}$ & $\begin{array}{l}--- \\
--- \\
--- \\
--- \\
---\end{array}$ & $\begin{array}{l}--- \\
--- \\
--- \\
--- \\
---\end{array}$ & $\begin{array}{l}6.6 \\
7.3 \\
6.2 \\
3.8 \\
2.5\end{array}$ & $\begin{array}{l}4.8 \\
5.2 \\
3.1 \\
2.1 \\
1.7\end{array}$ & $\begin{array}{l}5.8 \\
6.2 \\
4.4 \\
2.9 \\
2.1\end{array}$ \\
\hline $\begin{array}{l}21 \\
22 \\
23 \\
24 \\
25\end{array}$ & $\begin{array}{l}22.1 \\
22.1 \\
18.7 \\
17.8 \\
19.4\end{array}$ & $\begin{array}{l}18.4 \\
16.4 \\
16.1 \\
16.1 \\
13.0\end{array}$ & $\begin{array}{l}20.2 \\
19.0 \\
17.6 \\
17.1 \\
16.3\end{array}$ & $\begin{array}{l}--- \\
--- \\
--- \\
--- \\
---\end{array}$ & $\begin{array}{l}--- \\
--- \\
--- \\
--- \\
---\end{array}$ & $\begin{array}{l}--- \\
--- \\
--- \\
--- \\
---\end{array}$ & $\begin{array}{l}2.8 \\
2.1 \\
1.4 \\
1.4 \\
1.3\end{array}$ & $\begin{array}{l}1.7 \\
1.0 \\
0.4 \\
0.3 \\
0.3\end{array}$ & $\begin{array}{l}2.2 \\
1.5 \\
0.9 \\
0.8 \\
0.8\end{array}$ \\
\hline $\begin{array}{l}26 \\
27 \\
28 \\
29 \\
30 \\
31\end{array}$ & $\begin{array}{r}16.4 \\
17.5 \\
15.0 \\
13.8 \\
15.0 \\
---\end{array}$ & $\begin{array}{r}13.5 \\
12.8 \\
11.0 \\
9.3 \\
9.7 \\
---\end{array}$ & $\begin{array}{r}15.2 \\
15.0 \\
13.5 \\
11.7 \\
12.4 \\
---\end{array}$ & $\begin{array}{l}--- \\
--- \\
--- \\
--- \\
--- \\
---\end{array}$ & $\begin{array}{l}--- \\
--- \\
--- \\
--- \\
--- \\
---\end{array}$ & $\begin{array}{l}--- \\
--- \\
--- \\
--- \\
--- \\
---\end{array}$ & $\begin{array}{l}1.0 \\
2.1 \\
--- \\
--- \\
--- \\
---\end{array}$ & $\begin{array}{l}0.3 \\
0.9 \\
--- \\
--- \\
--- \\
---\end{array}$ & $\begin{array}{l}0.6 \\
1.7 \\
--- \\
--- \\
--- \\
---\end{array}$ \\
\hline MONTH & 27.8 & 9.3 & 18.1 & 21.0 & 5.0 & 13.4 & 10.2 & 0.3 & 5.5 \\
\hline
\end{tabular}


Table A16. Specific conductance and water temperature data collected May-November 2000 at station 102 (USGS identifier 442517068190501) Northeast Creek near Bar Harbor, ME - Continued.

DEEP

Temperature, water, degrees Celsius

\begin{tabular}{|c|c|c|c|c|c|c|c|c|c|c|c|c|}
\hline \multirow[t]{2}{*}{ DAY } & MAX & MIN & MEAN & MAX & MIN & MEAN & MAX & MIN & MEAN & MAX & MIN & MEAN \\
\hline & & MAY & & \multicolumn{3}{|c|}{ JUNE } & \multicolumn{3}{|c|}{ JULY } & \multicolumn{3}{|c|}{ AUGUST } \\
\hline 1 & --- & --- & --- & --- & --- & --- & --- & --- & --- & --- & --- & --- \\
\hline 2 & --- & --- & --- & --- & --- & --- & --- & --- & --- & --- & --- & --- \\
\hline 3 & --- & --- & --- & --- & --- & --- & --- & --- & --- & --- & --- & --- \\
\hline 4 & --- & --- & --- & --- & --- & --- & --- & --- & --- & --- & --- & --- \\
\hline 5 & --- & --- & --- & --- & --- & --- & --- & --- & --- & --- & --- & --- \\
\hline 6 & --- & --- & --- & --- & --- & --- & --- & --- & --- & --- & --- & --- \\
\hline 7 & --- & --- & --- & --- & --- & --- & 26.0 & 22.0 & 24.3 & --- & --- & --- \\
\hline 8 & --- & --- & --- & --- & --- & --- & 25.6 & 23.6 & 24.4 & 29.3 & 23.2 & 26.0 \\
\hline 9 & --- & --- & --- & --- & --- & --- & 26.5 & 22.0 & 23.9 & 28.8 & 24.8 & 26.3 \\
\hline 10 & --- & --- & --- & --- & --- & --- & -- & -- & -- & 28.3 & 23.6 & 25.6 \\
\hline 11 & --- & --- & --- & --- & --- & --- & --- & --- & --- & 26.9 & 21.0 & 24.2 \\
\hline 12 & --- & --- & --- & --- & --- & --- & --- & --- & --- & 26.5 & 21.7 & 23.6 \\
\hline 13 & --- & --- & --- & --- & --- & --- & --- & --- & --- & 24.8 & 22.1 & 23.5 \\
\hline 14 & --- & --- & --- & --- & --- & --- & --- & --- & --- & 25.2 & 23.2 & 23.8 \\
\hline 15 & --- & --- & --- & --- & --- & --- & --- & --- & --- & 25.2 & 23.2 & 24.1 \\
\hline 16 & --- & --- & --- & --- & --- & --- & --- & --- & --- & 24.8 & 23.6 & 24.1 \\
\hline 17 & --- & --- & --- & --- & --- & --- & --- & --- & --- & 26.0 & 22.8 & 23.9 \\
\hline 18 & --- & --- & --- & --- & --- & --- & --- & --- & --- & 25.2 & 19.3 & 23.0 \\
\hline 19 & --- & --- & --- & --- & --- & --- & --- & --- & --- & 25.2 & 22.4 & .4 \\
\hline 20 & --- & --- & --- & --- & --- & --- & --- & --- & --- & 24.4 & 20.6 & 22.7 \\
\hline 21 & --- & --- & --- & --- & --- & --- & --- & --- & --- & --- & --- & --- \\
\hline 22 & --- & --- & --- & --- & --- & --- & --- & --- & --- & --- & --- & --- \\
\hline 23 & --- & --- & --- & --- & --- & --- & --- & --- & --- & --- & --- & --- \\
\hline 24 & --- & --- & --- & --- & --- & --- & --- & --- & --- & --- & --- & --- \\
\hline 25 & --- & --- & --- & --- & --- & --- & --- & --- & --- & --- & --- & --- \\
\hline 26 & --- & --- & --- & --- & --- & --- & --- & --- & --- & --- & --- & --- \\
\hline 27 & --- & --- & --- & --- & --- & --- & -- & --- & --- & --- & --- & \\
\hline 28 & --- & --- & --- & --- & --- & --- & --- & --- & --- & --- & --- & \\
\hline 29 & --- & --- & --- & --- & --- & --- & --- & --- & --- & 24.4 & 18.4 & 20.9 \\
\hline 30 & --- & --- & --- & --- & --- & --- & --- & --- & --- & 22.1 & 18.1 & 20.0 \\
\hline 31 & --- & --- & --- & --- & --- & --- & --- & --- & --- & 25.6 & 19.0 & 22.1 \\
\hline MONTH & --- & --- & --- & --- & --- & --- & & & & 29.3 & 18.1 & 23.6 \\
\hline
\end{tabular}

\begin{tabular}{|c|c|c|c|c|c|c|c|c|c|}
\hline \multirow{2}{*}{ DAY } & MAX & MIN & MEAN & MAX & MIN & MEAN & MAX & MIN & MEAN \\
\hline & \multicolumn{3}{|c|}{ SEPTEMBER } & \multicolumn{3}{|c|}{ OCTOBER } & \multicolumn{3}{|c|}{ NOVEMBER } \\
\hline $\begin{array}{l}1 \\
2 \\
3 \\
4 \\
5\end{array}$ & $\begin{array}{l}27.4 \\
25.6 \\
22.1 \\
22.1 \\
24.4\end{array}$ & $\begin{array}{l}22.1 \\
21.3 \\
21.0 \\
21.0 \\
21.3\end{array}$ & $\begin{array}{l}24.6 \\
23.3 \\
21.3 \\
21.5 \\
22.5\end{array}$ & $\begin{array}{l}17.8 \\
18.4 \\
20.0 \\
19.3 \\
19.7\end{array}$ & $\begin{array}{l}12.8 \\
15.3 \\
17.5 \\
16.4 \\
17.5\end{array}$ & $\begin{array}{l}14.8 \\
16.9 \\
18.6 \\
18.5 \\
18.6\end{array}$ & $\begin{array}{l}6.9 \\
8.4 \\
9.0 \\
7.8 \\
6.9\end{array}$ & $\begin{array}{l}5.8 \\
6.4 \\
6.5 \\
6.0 \\
6.0\end{array}$ & $\begin{array}{l}6.4 \\
7.4 \\
7.8 \\
6.5 \\
6.4\end{array}$ \\
\hline $\begin{array}{r}6 \\
7 \\
8 \\
9 \\
10\end{array}$ & $\begin{array}{l}24.0 \\
22.4 \\
22.4 \\
25.6 \\
24.7\end{array}$ & $\begin{array}{l}20.6 \\
20.6 \\
19.0 \\
21.0 \\
21.0\end{array}$ & $\begin{array}{l}22.4 \\
21.6 \\
21.0 \\
23.3 \\
23.1\end{array}$ & $\begin{array}{l}18.7 \\
15.8 \\
15.8 \\
15.8 \\
13.5\end{array}$ & $\begin{array}{r}15.8 \\
14.5 \\
13.8 \\
13.5 \\
6.9\end{array}$ & $\begin{array}{r}17.2 \\
15.3 \\
15.3 \\
15.4 \\
9.4\end{array}$ & $\begin{array}{r}7.1 \\
8.8 \\
9.3 \\
10.4 \\
8.9\end{array}$ & $\begin{array}{l}6.2 \\
6.7 \\
8.0 \\
8.4 \\
7.6\end{array}$ & $\begin{array}{l}6.6 \\
7.6 \\
8.7 \\
9.3 \\
8.2\end{array}$ \\
\hline $\begin{array}{l}11 \\
12 \\
13 \\
14 \\
15\end{array}$ & $\begin{array}{l}23.5 \\
23.2 \\
24.0 \\
24.0 \\
22.0\end{array}$ & $\begin{array}{l}17.2 \\
19.4 \\
21.0 \\
19.0 \\
19.4\end{array}$ & $\begin{array}{l}21.1 \\
21.5 \\
22.4 \\
21.4 \\
20.6\end{array}$ & $\begin{array}{l}--- \\
--- \\
--- \\
--- \\
---\end{array}$ & $\begin{array}{l}--- \\
--- \\
--- \\
--- \\
---\end{array}$ & $\begin{array}{l}--- \\
--- \\
--- \\
--- \\
---\end{array}$ & $\begin{array}{l}9.2 \\
9.8 \\
9.1 \\
9.1 \\
9.2\end{array}$ & $\begin{array}{l}7.6 \\
8.6 \\
8.0 \\
8.4 \\
6.7\end{array}$ & $\begin{array}{l}8.4 \\
9.0 \\
8.6 \\
8.8 \\
8.5\end{array}$ \\
\hline $\begin{array}{l}16 \\
17 \\
18 \\
19 \\
20\end{array}$ & $\begin{array}{l}20.7 \\
20.0 \\
21.0 \\
20.7 \\
21.4\end{array}$ & $\begin{array}{l}17.5 \\
15.0 \\
17.5 \\
18.1 \\
20.0\end{array}$ & $\begin{array}{l}19.0 \\
17.7 \\
19.1 \\
20.1 \\
20.6\end{array}$ & $\begin{array}{l}--- \\
--- \\
--- \\
--- \\
---\end{array}$ & $\begin{array}{l}--- \\
--- \\
--- \\
--- \\
---\end{array}$ & $\begin{array}{l}--- \\
--- \\
--- \\
--- \\
---\end{array}$ & $\begin{array}{l}6.7 \\
7.1 \\
6.2 \\
3.8 \\
2.5\end{array}$ & $\begin{array}{l}4.8 \\
5.2 \\
3.1 \\
2.1 \\
1.7\end{array}$ & $\begin{array}{l}5.9 \\
6.2 \\
4.5 \\
2.9 \\
2.1\end{array}$ \\
\hline $\begin{array}{l}21 \\
22 \\
23 \\
24 \\
25\end{array}$ & $\begin{array}{l}22.4 \\
22.1 \\
19.4 \\
18.4 \\
19.4\end{array}$ & $\begin{array}{l}20.3 \\
17.5 \\
18.1 \\
16.7 \\
15.5\end{array}$ & $\begin{array}{l}21.1 \\
19.8 \\
18.6 \\
17.5 \\
17.8\end{array}$ & $\begin{array}{l}--- \\
--- \\
--- \\
--- \\
---\end{array}$ & $\begin{array}{l}--- \\
--- \\
--- \\
--- \\
---\end{array}$ & $\begin{array}{l}--- \\
--- \\
--- \\
--- \\
---\end{array}$ & $\begin{array}{l}2.8 \\
2.1 \\
--- \\
--- \\
---\end{array}$ & $\begin{array}{l}1.8 \\
1.1 \\
--- \\
--- \\
---\end{array}$ & $\begin{array}{l}2.2 \\
1.6 \\
--- \\
--- \\
---\end{array}$ \\
\hline $\begin{array}{l}26 \\
27 \\
28 \\
29 \\
30 \\
31\end{array}$ & $\begin{array}{r}15.8 \\
17.2 \\
15.0 \\
14.0 \\
15.3 \\
---\end{array}$ & $\begin{array}{r}14.0 \\
14.0 \\
12.1 \\
11.0 \\
10.6 \\
---\end{array}$ & $\begin{array}{r}15.3 \\
15.3 \\
14.0 \\
12.5 \\
13.2 \\
---\end{array}$ & $\begin{array}{l}--- \\
--- \\
--- \\
--- \\
--- \\
---\end{array}$ & $\begin{array}{l}--- \\
--- \\
--- \\
--- \\
--- \\
---\end{array}$ & $\begin{array}{l}--- \\
--- \\
--- \\
--- \\
--- \\
---\end{array}$ & $\begin{array}{l}--- \\
2.1 \\
--- \\
--- \\
--- \\
---\end{array}$ & $\begin{array}{l}--- \\
1.0 \\
--- \\
--- \\
--- \\
---\end{array}$ & $\begin{array}{l}--- \\
1.7 \\
--- \\
--- \\
--- \\
--\end{array}$ \\
\hline MONTH & 27.4 & 10.6 & 19.8 & 20.0 & 6.9 & 16.0 & 10.4 & 1.0 & 6.3 \\
\hline
\end{tabular}


Table A17. Specific conductance and water temperature data collected May-November 2000 at station 103 (USGS identifier 442507068185301) Northeast Creek near Bar Harbor, ME [Data are presented as minimum, mean and maximum daily values of continuous data at 15-minute intervals. Shallow and deep data were collected at fixed depths located, respectively, about $0.2 \mathrm{~m}$ below the water surface and about $0.2 \mathrm{~m}$ above the stream bottom.]

SHALLOW

Specific conductance, water, microsiemens per centimeter at 25 degrees Celsius

\begin{tabular}{|c|c|c|c|c|c|c|c|c|c|c|c|c|}
\hline \multirow[t]{2}{*}{ DAY } & MAX & MIN & MEAN & MAX & MIN & MEAN & MAX & MIN & MEAN & MAX & MIN & MEAN \\
\hline & & MAY & & \multicolumn{3}{|c|}{ JUNE } & \multicolumn{3}{|c|}{ JULY } & \multicolumn{3}{|c|}{ AUGUST } \\
\hline 1 & --- & --- & --- & 3570 & 264 & 391 & 36100 & 18200 & 27600 & --- & --- & --- \\
\hline 2 & --- & --- & --- & 7000 & 455 & 1680 & 41400 & 24600 & 33200 & --- & --- & --- \\
\hline 3 & --- & --- & --- & --- & --- & --- & 43500 & 25900 & 34800 & --- & --- & --- \\
\hline $\begin{array}{l}4 \\
5\end{array}$ & $\begin{array}{l}--- \\
---\end{array}$ & $\begin{array}{l}--- \\
---\end{array}$ & $\begin{array}{l}--- \\
---\end{array}$ & $\begin{array}{l}--- \\
---\end{array}$ & --- & $\begin{array}{l}--- \\
---\end{array}$ & $\begin{array}{l}41600 \\
39400\end{array}$ & $\begin{array}{l}25900 \\
27900\end{array}$ & $\begin{array}{l}33800 \\
3332\end{array}$ & --- & --- & $\begin{array}{l}--- \\
---\end{array}$ \\
\hline & & & - & -- & --- & -- & 39400 & 27900 & 33300 & -- & --- & -- \\
\hline 6 & --- & --- & --- & --- & --- & --- & 33200 & 21800 & 28100 & --- & --- & - \\
\hline 7 & --- & --- & --- & --- & --- & --- & 23300 & 8420 & 15300 & --- & --- & \\
\hline 8 & --- & --- & --- & --- & --- & --- & 12400 & 4950 & 7190 & --- & --- & - \\
\hline 9 & --- & --- & --- & --- & --- & --- & 16700 & 6900 & 11900 & --- & --- & - \\
\hline 10 & --- & --- & --- & --- & --- & --- & --- & --- & --- & --- & --- & - \\
\hline 11 & --- & --- & --- & --- & --- & --- & --- & --- & --- & 20600 & 18600 & 1960 \\
\hline 12 & --- & --- & --- & & --- & --- & --- & --- & --- & 19500 & 18700 & 19000 \\
\hline 13 & --- & --- & --- & --- & --- & --- & --- & --- & --- & 19400 & 18900 & 19200 \\
\hline 14 & --- & --- & --- & 1260 & 746 & 1050 & --- & --- & --- & 20700 & 19100 & 1990 \\
\hline 15 & --- & --- & --- & 1430 & 999 & 1230 & --- & --- & --- & 21400 & 20200 & 20900 \\
\hline 16 & --- & --- & --- & 1500 & 1010 & 1270 & --- & --- & --- & 22500 & 20700 & 21700 \\
\hline 17 & --- & --- & --- & 1460 & 1060 & 1310 & --- & --- & --- & 23500 & 21600 & 22600 \\
\hline 18 & 292 & 195 & 234 & 1310 & 927 & 1140 & --- & --- & --- & 22600 & 21600 & 22000 \\
\hline 19 & 284 & 114 & 161 & 1310 & 683 & 1020 & --- & --- & --- & 22000 & 20900 & 21500 \\
\hline 20 & 165 & 110 & 131 & 1100 & 803 & 940 & --- & --- & --- & 21500 & 19600 & 20900 \\
\hline 21 & 236 & 140 & 174 & 1260 & 601 & 1050 & --- & --- & --- & --- & --- & 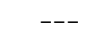 \\
\hline 22 & 383 & 192 & 250 & 1340 & 1060 & 1230 & --- & --- & --- & --- & --- & \\
\hline 23 & 394 & 257 & 292 & 1260 & 1040 & 1110 & --- & --- & --- & --- & --- & \\
\hline 24 & 273 & 174 & 228 & 1230 & 601 & 1050 & --- & --- & --- & --- & --- & \\
\hline 25 & 174 & 67 & 92 & 1280 & 910 & 1150 & --- & --- & --- & --- & --- & \\
\hline 26 & 83 & 64 & 69 & 1270 & 638 & 1040 & --- & --- & --- & --- & --- & -- \\
\hline 27 & 108 & 76 & 87 & 1230 & 967 & 1100 & --- & --- & --- & --- & --- & \\
\hline 28 & 131 & 92 & 101 & 2700 & 1130 & 1280 & --- & --- & --- & --- & --- & \\
\hline 29 & 165 & 107 & 126 & 25300 & 2120 & 4320 & --- & --- & --- & 43200 & 37500 & 41000 \\
\hline 30 & 255 & 139 & 187 & 34700 & 8880 & 18200 & --- & --- & --- & 44100 & 40900 & 42900 \\
\hline 31 & 368 & 217 & 294 & --- & --- & --- & --- & --- & --- & 43700 & 39500 & 42000 \\
\hline MONTH & 394 & 64 & 173 & 34700 & 264 & 2190 & 43500 & 4950 & 25000 & 44100 & 18600 & 25600 \\
\hline \multirow[t]{2}{*}{ DAY } & MAX & MIN & MEAN & MAX & MIN & MEAN & & & & & & \\
\hline & \multicolumn{3}{|c|}{ SEPTEMBER } & \multicolumn{3}{|c|}{ OCTOBER } & & & & & & \\
\hline 1 & 42100 & 38500 & 41200 & 35800 & 32200 & 34000 & & & & & & \\
\hline 2 & 40600 & 30600 & 37100 & 33500 & 28800 & 30700 & & & & & & \\
\hline $\begin{array}{l}3 \\
4\end{array}$ & 36000 & 31100 & 34900 & $\begin{array}{l}31700 \\
300\end{array}$ & 27900 & 29700 & & & & & & \\
\hline $\begin{array}{l}4 \\
5\end{array}$ & $\begin{array}{l}34600 \\
28600\end{array}$ & 23500 & $\begin{array}{l}30100 \\
24500\end{array}$ & $\begin{array}{l}30800 \\
28800\end{array}$ & $\begin{array}{l}28600 \\
25000\end{array}$ & $\begin{array}{l}29500 \\
26500\end{array}$ & & & & & & \\
\hline 6 & 25700 & 23700 & 24600 & 26300 & 18800 & 20600 & & & & & & \\
\hline 7 & 26400 & 23800 & 25100 & 18800 & 14900 & 16400 & & & & & & \\
\hline 8 & 24900 & 22600 & 23700 & 17400 & 14000 & 15300 & & & & & & \\
\hline 9 & 24600 & 22800 & 23700 & 14600 & 8990 & 11700 & & & & & & \\
\hline 10 & 23600 & 22200 & 23000 & 12800 & 9090 & 10800 & & & & & & \\
\hline 11 & 24000 & 22200 & 23400 & --- & --- & --- & & & & & & \\
\hline 12 & 24700 & 23500 & 24100 & --- & --- & --- & & & & & & \\
\hline 13 & 27100 & 24300 & 25400 & --- & --- & --- & & & & & & \\
\hline 14 & 27000 & 24600 & 25700 & --- & --- & --- & & & & & & \\
\hline 15 & 29400 & 25700 & 27400 & --- & --- & --- & & & & & & \\
\hline 16 & 33300 & 27400 & 31100 & --- & --- & --- & & & & & & \\
\hline 17 & 31900 & 29700 & 31000 & --- & --- & --- & & & & & & \\
\hline 18 & 30400 & 26800 & 28200 & --- & --- & --- & & & & & & \\
\hline 19 & 28300 & 25400 & 26400 & --- & --- & --- & & & & & & \\
\hline 20 & 27200 & 24200 & 25400 & --- & --- & --- & & & & & & \\
\hline 21 & 29900 & 25400 & 26300 & --- & --- & --- & & & & & & \\
\hline 22 & 30300 & 27200 & 28600 & --- & --- & --- & & & & & & \\
\hline 23 & 28500 & 26600 & 27600 & --- & --- & --- & & & & & & \\
\hline 24 & 30200 & 26900 & 28600 & --- & --- & --- & & & & & & \\
\hline 25 & 33300 & 27100 & 28800 & --- & --- & --- & & & & & & \\
\hline 26 & 38900 & 31400 & 33900 & --- & --- & --- & & & & & & \\
\hline 27 & 41700 & 37300 & 40000 & --- & --- & --- & & & & & & \\
\hline 28 & 42200 & 36000 & 40300 & --- & --- & --- & & & & & & \\
\hline 29 & 38700 & 34500 & 37500 & --- & --- & --- & & & & & & \\
\hline 30 & 37800 & 34600 & 36000 & --- & --- & --- & & & & & & \\
\hline 31 & & & & --- & --- & --- & & & & & & \\
\hline MONTH & 42200 & 22200 & 29500 & 35800 & 8990 & 22500 & & & & & & \\
\hline
\end{tabular}


Table A17. Specific conductance and water temperature data collected May-November 2000 at station 103 (USGS identifier 442507068185301) Northeast Creek near Bar Harbor, ME - Continued.

DEEP

Specific conductance, water, microsiemens per centimeter at 25 degrees Celsius

\begin{tabular}{|c|c|c|c|c|c|c|c|c|c|c|c|c|}
\hline \multirow[t]{2}{*}{ DAY } & MAX & MIN & MEAN & MAX & MIN & MEAN & MAX & MIN & MEAN & MAX & MIN & MEAN \\
\hline & & MAY & & \multicolumn{3}{|c|}{ JUNE } & \multicolumn{3}{|c|}{ JULY } & \multicolumn{3}{|c|}{ AUGUST } \\
\hline 1 & --- & --- & --- & 3760 & 286 & 400 & 36100 & 32600 & 34000 & --- & --- & --- \\
\hline 2 & --- & --- & --- & 7000 & 462 & 2000 & 41100 & 33500 & 39100 & --- & --- & --- \\
\hline 3 & --- & --- & --- & --- & --- & --- & 44100 & 35500 & 41200 & --- & --- & --- \\
\hline 4 & --- & --- & --- & --- & --- & --- & 42400 & 32300 & 40000 & --- & --- & --- \\
\hline 5 & --- & --- & --- & --- & --- & --- & 39900 & 34500 & 37900 & --- & --- & --- \\
\hline 6 & --- & --- & --- & --- & --- & --- & 35600 & 31300 & 34400 & --- & --- & --- \\
\hline 7 & --- & --- & --- & --- & --- & --- & 31300 & 19200 & 24500 & --- & --- & --- \\
\hline 8 & --- & --- & --- & --- & --- & --- & 20000 & 17500 & 19300 & --- & --- & --- \\
\hline 9 & --- & --- & --- & --- & --- & --- & 21100 & 15000 & 18800 & --- & --- & --- \\
\hline 10 & --- & --- & --- & --- & --- & --- & --- & --- & --- & --- & --- & --- \\
\hline 11 & --- & --- & --- & --- & --- & --- & --- & --- & --- & 22900 & 20600 & 21400 \\
\hline 12 & --- & --- & --- & --- & --- & --- & --- & --- & --- & 21100 & 19700 & 20500 \\
\hline 13 & --- & --- & --- & --- & --- & --- & --- & --- & --- & 20400 & 19500 & 19800 \\
\hline 14 & --- & --- & --- & 1430 & 934 & 1240 & --- & --- & --- & 21800 & 19400 & 21200 \\
\hline 15 & --- & --- & --- & 1460 & 1140 & 1320 & --- & --- & --- & 22100 & 21100 & 21600 \\
\hline 16 & --- & --- & --- & 1680 & 1240 & 1400 & --- & --- & --- & 22700 & 21900 & 22300 \\
\hline 17 & --- & --- & --- & 1630 & 1340 & 1480 & --- & --- & --- & 23200 & 22000 & 22800 \\
\hline 18 & 5890 & 4680 & 5540 & 1530 & 1240 & 1350 & --- & --- & --- & 23100 & 22100 & 22500 \\
\hline 19 & 5800 & 5560 & 5650 & 1480 & 1150 & 1260 & --- & --- & --- & 22500 & 21800 & 22100 \\
\hline 20 & 5600 & 5380 & 5480 & 1380 & 933 & 1120 & --- & --- & --- & 22200 & 21200 & 21700 \\
\hline 21 & 5460 & 5340 & 5400 & 1370 & 1060 & 1200 & --- & --- & --- & --- & --- & - \\
\hline 22 & 5390 & 3340 & 4990 & 1430 & 1240 & 1310 & --- & --- & --- & --- & --- & --- \\
\hline 23 & 5520 & 4890 & 5310 & 1480 & 1100 & 1230 & --- & --- & --- & --- & --- & --- \\
\hline 24 & 5410 & 1120 & 3650 & 1410 & 1150 & 1250 & --- & --- & --- & --- & --- & --- \\
\hline 25 & 5310 & 2750 & 4560 & 1520 & 1170 & 1270 & --- & --- & --- & --- & --- & --- \\
\hline 26 & 5380 & 5190 & 5260 & 1610 & 1360 & 1510 & --- & --- & --- & --- & --- & --- \\
\hline 27 & 5350 & 4970 & 5220 & 1430 & 1200 & 1290 & --- & --- & --- & --- & --- & --- \\
\hline 28 & 5190 & 4840 & 5040 & $\begin{array}{r}2070 \\
25500\end{array}$ & 1300 & 1470 & -- & --- & -- & --- & 3930-- & ק--- \\
\hline $\begin{array}{l}29 \\
30\end{array}$ & 5040 & $\begin{array}{l}4630 \\
4620\end{array}$ & 4790 & $\begin{array}{l}25500 \\
34900\end{array}$ & $\begin{array}{r}2070 \\
21400\end{array}$ & $\begin{array}{r}4430 \\
23700\end{array}$ & --- & - & -_- & $\begin{array}{l}43400 \\
45000\end{array}$ & $\begin{array}{l}39300 \\
42000\end{array}$ & $\begin{array}{l}42000 \\
43800\end{array}$ \\
\hline 31 & 4830 & 317 & 2810 & 54500 & & 20100 & --- & --- & --- & 44700 & 42000 & 43200 \\
\hline MONTH & 5890 & 317 & 4880 & 34900 & 286 & 2640 & 44100 & 15000 & 32100 & 45000 & 19400 & 26500 \\
\hline \multirow[t]{2}{*}{ DAY } & MAX & MIN & MEAN & MAX & MIN & MEAN & & & & & & \\
\hline & \multicolumn{3}{|c|}{ SEPTEMBER } & \multicolumn{3}{|c|}{ OCTOBER } & & & & & & \\
\hline 1 & 43000 & 41000 & 42100 & 39400 & 36400 & 37900 & & & & & & \\
\hline 2 & 42200 & 36200 & 40500 & 37400 & 35000 & $\begin{array}{l}36300 \\
35500\end{array}$ & & & & & & \\
\hline $\begin{array}{l}3 \\
4\end{array}$ & $\begin{array}{l}40700 \\
39000\end{array}$ & $\begin{array}{l}37600 \\
36300\end{array}$ & $\begin{array}{l}39000 \\
37400\end{array}$ & $\begin{array}{l}365000 \\
35800\end{array}$ & $\begin{array}{l}34500 \\
33900\end{array}$ & $\begin{array}{l}35500 \\
34800\end{array}$ & & & & & & \\
\hline 5 & 37100 & 34700 & 35900 & 34700 & 32400 & 33600 & & & & & & \\
\hline 6 & 35600 & 33300 & 34300 & 33600 & 29600 & 31200 & & & & & & \\
\hline 7 & 33800 & 31100 & 32400 & 29800 & 27100 & 28300 & & & & & & \\
\hline 8 & 31300 & 28900 & 29800 & 27800 & 26400 & 27000 & & & & & & \\
\hline $\begin{array}{r}9 \\
10\end{array}$ & $\begin{array}{l}29600 \\
28300\end{array}$ & 26600 & $\begin{array}{l}28100 \\
26500\end{array}$ & $\begin{array}{l}27000 \\
25800\end{array}$ & 25400 & 26400 & & & & & & \\
\hline & & & s & & & & & & & & & \\
\hline $\begin{array}{l}11 \\
12\end{array}$ & 26500 & 23800 & 24800 & --- & --- & --- & & & & & & \\
\hline 13 & 27900 & 25200 & 27200 & --- & --- & --- & & & & & & \\
\hline 14 & 27400 & 26300 & 26900 & --- & --- & --- & & & & & & \\
\hline 15 & 30600 & 26600 & 28700 & --- & --- & --- & & & & & & \\
\hline 16 & 33900 & 30000 & 31900 & --- & --- & --- & & & & & & \\
\hline 17 & 33000 & 31500 & 32100 & --- & --- & --- & & & & & & \\
\hline 18 & 32400 & 30400 & 31300 & --- & --- & --- & & & & & & \\
\hline $\begin{array}{l}19 \\
20\end{array}$ & 31400 & 29500 & 30800 & $\begin{array}{l}--- \\
---\end{array}$ & $\begin{array}{l}--- \\
---\end{array}$ & --- & & & & & & \\
\hline & & & & & & & & & & & & \\
\hline 21 & 30400 & 28100 & 29600 & --- & --- & --- & & & & & & \\
\hline 22 & 30000 & 29300 & 29700 & --- & --- & --- & & & & & & \\
\hline 23 & 29900 & 28200 & 28900 & --- & --- & --- & & & & & & \\
\hline $\begin{array}{l}24 \\
25\end{array}$ & 30900 & 28900 & 30400 & --- & $\begin{array}{l}--- \\
---\end{array}$ & $\begin{array}{l}--- \\
---\end{array}$ & & & & & & \\
\hline & (35900 & 29800 & 30600 & & & & & & & & & \\
\hline 26 & 40100 & 32900 & 34600 & --- & --- & --- & & & & & & \\
\hline 27 & 42300 & 39300 & 40700 & --- & --- & --- & & & & & & \\
\hline 28 & 43500 & 40400 & 41500 & --- & --- & --- & & & & & & \\
\hline 29 & 42400 & 38700 & 40100 & --- & --- & --- & & & & & & \\
\hline 30 & 40100 & 38200 & 39000 & --- & --- & --- & & & & & & \\
\hline 31 & -- & -- & -- & --- & --- & --- & & & & & & \\
\hline MONTH & 43500 & 23800 & 32600 & 39400 & 23900 & 31600 & & & & & & \\
\hline
\end{tabular}


Table A17. Specific conductance and water temperature data collected May-November 2000 at station 103 (USGS identifier 442507068185301) Northeast Creek near Bar Harbor, ME - Continued.

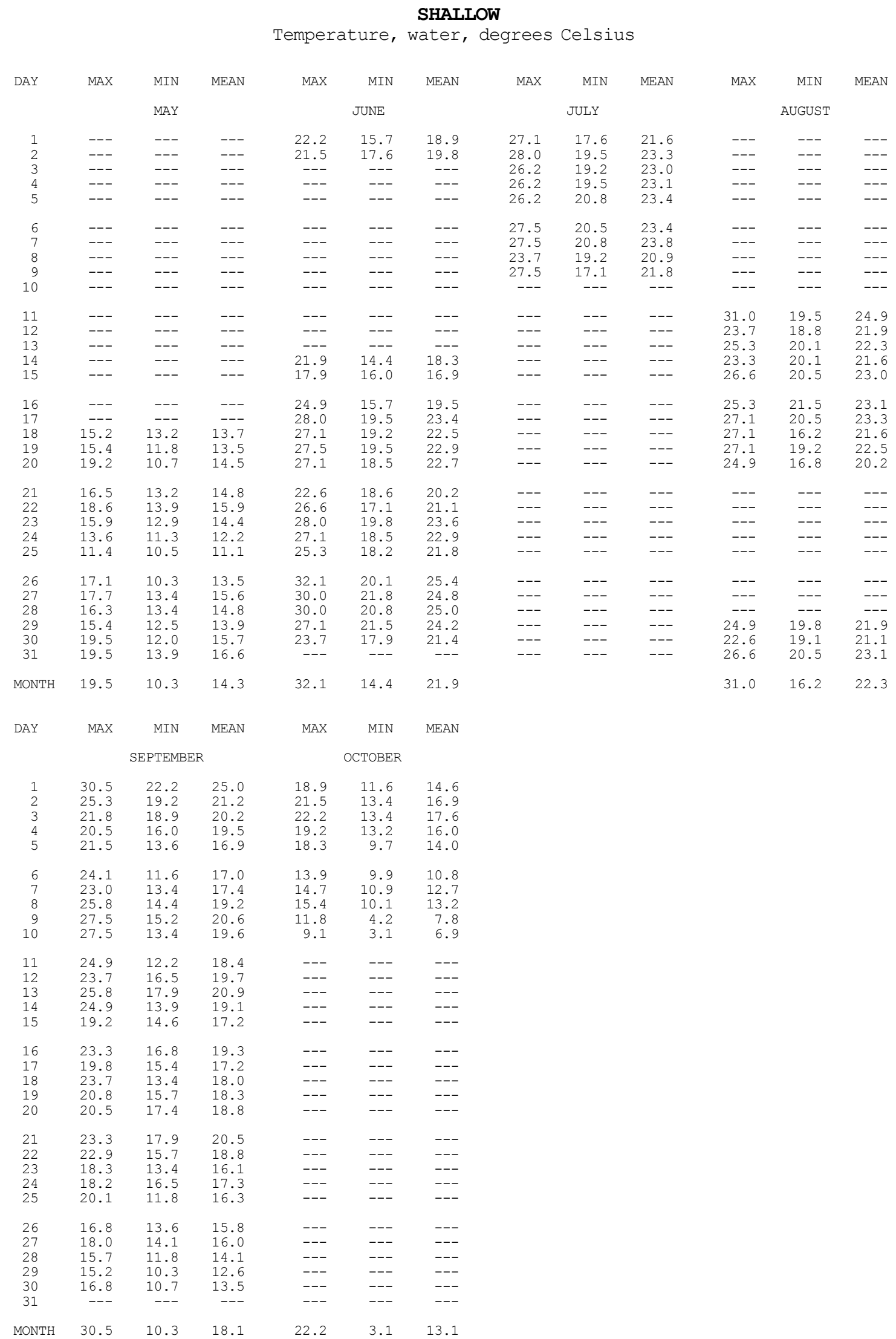


Table A17. Specific conductance and water temperature data collected May-November 2000 at station 103 (USGS identifier 442507068185301) Northeast Creek near Bar Harbor, ME - Continued.

DEEP

Temperature, water, degrees Celsius

\begin{tabular}{|c|c|c|c|c|c|c|c|c|c|c|c|c|}
\hline \multirow[t]{2}{*}{ DAY } & MAX & MIN & MEAN & MAX & MIN & MEAN & MAX & MIN & MEAN & MAX & MIN & MEAN \\
\hline & & MAY & & \multicolumn{3}{|c|}{ JUNE } & \multicolumn{3}{|c|}{ JULY } & \multicolumn{3}{|c|}{ AUGUST } \\
\hline 1 & --- & --- & --- & 22.2 & 15.7 & 18.9 & 26.2 & 17.6 & 21.7 & --- & --- & --- \\
\hline 2 & --- & -- & --- & 21.5 & 17.9 & 19.9 & 28.0 & 19.8 & 23.6 & --- & --- & \\
\hline 3 & --- & --- & --- & --- & --- & --- & 25.8 & 19.5 & 22.9 & --- & --- & - \\
\hline $\begin{array}{l}4 \\
5\end{array}$ & $\begin{array}{l}--- \\
---\end{array}$ & --- & $\begin{array}{l}--- \\
---\end{array}$ & --- & --- & --- & 26.2 & 19.5 & 23.3 & --- & --- & --- \\
\hline & & & & -- & -- & -- & 27.1 & 21.1 & 24.1 & -- & -- & - \\
\hline 6 & --- & --- & --- & --- & --- & --- & 30.0 & 21.5 & 24.6 & --- & --- & --- \\
\hline 7 & --- & --- & --- & --- & --- & --- & 29.5 & 23.7 & 25.6 & --- & --- & \\
\hline 8 & --- & --- & --- & --- & --- & --- & 24.5 & 20.8 & 22.8 & --- & --- & \\
\hline 9 & --- & --- & --- & --- & --- & --- & 26.6 & 17.9 & 22.5 & --- & --- & \\
\hline 10 & --- & --- & --- & --- & --- & --- & -- & -- & -- & --- & --- & - \\
\hline 11 & --- & --- & --- & --- & --- & --- & --- & --- & --- & 29.0 & 21.1 & 25.0 \\
\hline 12 & --- & --- & --- & --- & --- & --- & --- & --- & --- & 24.9 & 20.1 & 22.7 \\
\hline 13 & --- & --- & --- & --- & --- & --- & --- & --- & --- & 24.5 & 20.5 & 22.5 \\
\hline 14 & --- & --- & --- & 21.9 & 14.1 & 18.3 & --- & --- & --- & 23.7 & 21.1 & 22 \\
\hline 15 & --- & --- & --- & 17.9 & 16.2 & 16.9 & --- & --- & --- & 25.3 & 22.6 & 23.7 \\
\hline 16 & --- & --- & --- & 23.3 & 15.7 & 18.8 & --- & --- & --- & 25.3 & 23.3 & 24.2 \\
\hline 17 & - & --- & --- & 27.1 & 19.5 & 22.7 & --- & --- & --- & 26.2 & 21.8 & 24.1 \\
\hline 18 & 15.2 & 13.2 & 13.8 & 25.3 & 18.8 & 22.1 & --- & --- & --- & 24.5 & 17.9 & 21.4 \\
\hline 19 & 15.4 & 11.8 & 13.6 & 26.2 & 19.5 & 22.2 & --- & --- & --- & 24.1 & 19.8 & 22.3 \\
\hline 20 & 18.9 & 10.7 & 14.5 & 27.1 & 17.9 & 22.2 & --- & --- & --- & 22.6 & 17.9 & 20.5 \\
\hline 21 & 16.5 & 13.4 & 14.9 & 22.2 & 17.9 & 19.9 & --- & --- & --- & --- & --- & - \\
\hline 22 & 18.6 & 13.9 & 16.0 & 23.7 & 17.4 & 20.2 & --- & --- & --- & --- & --- & \\
\hline 23 & 15.9 & 12.7 & 14.4 & 27.1 & 19.5 & 23.1 & --- & --- & --- & --- & --- & \\
\hline 24 & 13.9 & 11.3 & 12.3 & 24.9 & 17.6 & 21.7 & --- & --- & --- & --- & --- & \\
\hline 25 & 11.6 & 10.7 & 11.1 & 25.3 & 17.4 & 21.5 & --- & --- & --- & --- & --- & --- \\
\hline 26 & 17.1 & 10.3 & 13.5 & 30.0 & 20.1 & 24.5 & --- & --- & --- & --- & --- & --- \\
\hline 27 & 18.0 & 13.4 & 15.7 & 26.2 & 21.8 & 24.2 & --- & --- & --- & --- & --- & --- \\
\hline $\begin{array}{l}28 \\
29\end{array}$ & $\begin{array}{l}16 \cdot 5 \\
15\end{array}$ & 13.4 & $\begin{array}{l}14.9 \\
14\end{array}$ & 28.0 & 20.1 & 24.1 & --- & --- & --- & 237 & $---\overline{8}$ & 21.9 \\
\hline 30 & $\begin{array}{l}19.4 \\
19.5\end{array}$ & $\begin{array}{l}12.5 \\
12.0\end{array}$ & $\begin{array}{l}14.0 \\
15.7\end{array}$ & $\begin{array}{l}26.6 \\
23.7\end{array}$ & $\begin{array}{l}21.1 \\
17.9\end{array}$ & $\begin{array}{l}24.1 \\
22.4\end{array}$ & --- & --- & --- & 22.2 & 20.1 & 21.2 \\
\hline 31 & 19.2 & 13.9 & 16.6 & -- & -- & -- & --- & --- & --- & 24.5 & 20.5 & 22.4 \\
\hline MONTH & 19.5 & 10.3 & 14.4 & 30.0 & 14.1 & 21.5 & 30.0 & 17.6 & 23.5 & 29.0 & 17.9 & 22.7 \\
\hline
\end{tabular}

\begin{tabular}{|c|c|c|c|c|c|c|}
\hline \multirow[t]{2}{*}{ DAY } & MAX & MIN & MEAN & MAX & MIN & MEAN \\
\hline & \multicolumn{3}{|c|}{ SEPTEMBER } & \multicolumn{3}{|c|}{ OCTOBER } \\
\hline $\begin{array}{l}1 \\
2 \\
3 \\
4 \\
5\end{array}$ & $\begin{array}{l}25.8 \\
25.3 \\
21.5 \\
20.8 \\
20.5\end{array}$ & $\begin{array}{l}22.6 \\
20.1 \\
19.8 \\
19.5 \\
16.5\end{array}$ & $\begin{array}{l}24.2 \\
21.6 \\
20.8 \\
20.3 \\
18.8\end{array}$ & $\begin{array}{l}16.8 \\
17.7 \\
18.0 \\
17.9 \\
16.5\end{array}$ & $\begin{array}{l}12.9 \\
14.4 \\
15.2 \\
14.7 \\
13.2\end{array}$ & $\begin{array}{l}15.0 \\
16.0 \\
16.6 \\
16.3 \\
15.1\end{array}$ \\
\hline $\begin{array}{r}6 \\
7 \\
8 \\
9 \\
10\end{array}$ & $\begin{array}{l}21.2 \\
21.5 \\
22.6 \\
23.3 \\
23.0\end{array}$ & $\begin{array}{l}15.7 \\
15.9 \\
17.1 \\
17.9 \\
15.7\end{array}$ & $\begin{array}{l}18.8 \\
18.9 \\
19.9 \\
20.7 \\
19.5\end{array}$ & $\begin{array}{l}15.7 \\
14.7 \\
15.7 \\
14.4 \\
10.5\end{array}$ & $\begin{array}{r}11.6 \\
12.0 \\
12.5 \\
8.5 \\
7.0\end{array}$ & $\begin{array}{r}12.5 \\
13.2 \\
14.3 \\
11.0 \\
9.1\end{array}$ \\
\hline $\begin{array}{l}11 \\
12 \\
13 \\
14 \\
15\end{array}$ & $\begin{array}{l}21.9 \\
21.5 \\
23.0 \\
21.5 \\
19.5\end{array}$ & $\begin{array}{l}14.6 \\
17.4 \\
19.5 \\
16.2 \\
15.4\end{array}$ & $\begin{array}{l}18.5 \\
19.5 \\
21.0 \\
19.3 \\
18.0\end{array}$ & $\begin{array}{l}--- \\
--- \\
--- \\
--- \\
---\end{array}$ & $\begin{array}{l}--- \\
--- \\
--- \\
--- \\
---\end{array}$ & $\begin{array}{l}--- \\
--- \\
--- \\
--- \\
--\end{array}$ \\
\hline $\begin{array}{l}16 \\
17 \\
18 \\
19 \\
20\end{array}$ & $\begin{array}{l}21.5 \\
19.2 \\
21.2 \\
20.1 \\
19.8\end{array}$ & $\begin{array}{l}17.6 \\
15.9 \\
14.1 \\
15.7 \\
17.9\end{array}$ & $\begin{array}{l}19.6 \\
17.7 \\
18.0 \\
18.1 \\
18.8\end{array}$ & $\begin{array}{l}--- \\
--- \\
--- \\
--- \\
---\end{array}$ & $\begin{array}{l}--- \\
--- \\
--- \\
--- \\
--\end{array}$ & $\begin{array}{l}--- \\
--- \\
--- \\
--- \\
--\end{array}$ \\
\hline $\begin{array}{l}21 \\
22 \\
23 \\
24 \\
25\end{array}$ & $\begin{array}{l}21.9 \\
20.8 \\
18.3 \\
18.9 \\
18.9\end{array}$ & $\begin{array}{l}18.9 \\
16.8 \\
14.1 \\
16.8 \\
14.9\end{array}$ & $\begin{array}{l}20.3 \\
19.3 \\
16.3 \\
18.0 \\
17.1\end{array}$ & $\begin{array}{l}--- \\
--- \\
--- \\
--- \\
---\end{array}$ & $\begin{array}{l}--- \\
--- \\
--- \\
--- \\
---\end{array}$ & $\begin{array}{l}--- \\
--- \\
--- \\
--- \\
--\end{array}$ \\
\hline $\begin{array}{l}26 \\
27 \\
28 \\
29 \\
30 \\
31\end{array}$ & $\begin{array}{l}16.8 \\
17.6 \\
15.7 \\
14.9 \\
15.4\end{array}$ & $\begin{array}{l}14.6 \\
14.9 \\
13.2 \\
11.8 \\
13.2\end{array}$ & $\begin{array}{l}15.9 \\
16.1 \\
14.7 \\
13.5 \\
14.5\end{array}$ & $\begin{array}{l}--- \\
--- \\
--- \\
--- \\
--- \\
---\end{array}$ & $\begin{array}{l}--- \\
--- \\
--- \\
--- \\
---\end{array}$ & $\begin{array}{l}--- \\
--- \\
--- \\
--- \\
--\end{array}$ \\
\hline MONTH & 25.8 & 11.8 & 18.6 & 18.0 & 7.0 & 3.9 \\
\hline
\end{tabular}


Hydrologic Data Summary for the Northeast Creek/Fresh Meadow Estuary, Acadia National Park, Maine, 2000-2001

Table A18. Specific conductance and water temperature data collected May-November 2000 at station 104 (USGS identifier 442509068181901) Northeast Creek near Bar Harbor, ME [Data are presented as minimum, mean and maximum daily values of continuous data at 15-minute intervals. Shallow and deep data were collected at fixed depths located, respectively, about $0.2 \mathrm{~m}$ below the water surface and about $0.2 \mathrm{~m}$ above the stream bottom.]

SHALLOW

Specific conductance, water, microsiemens per centimeter at 25 degrees Celsius

\begin{tabular}{|c|c|c|c|c|c|c|c|c|c|c|c|c|}
\hline DAY & MAX & $\begin{array}{l}\text { MIN } \\
\text { MAY }\end{array}$ & MEAN & MAX & $\begin{array}{l}\text { MIN } \\
\text { JUNE }\end{array}$ & MEAN & MAX & $\begin{array}{l}\text { MIN } \\
\text { JULY }\end{array}$ & MEAN & MAX & $\begin{array}{c}\text { MIN } \\
\text { AUGUST }\end{array}$ & MEAN \\
\hline 1 & --- & --- & --- & 256 & 98 & 113 & 23700 & 1240 & 8570 & --- & --- & --- \\
\hline 2 & --- & --- & --- & 428 & 113 & 146 & 28600 & 3290 & 16900 & --- & --- & --- \\
\hline 3 & --- & --- & --- & --- & --- & --- & 33300 & 19300 & 26400 & --- & --- & --- \\
\hline $\begin{array}{l}4 \\
5\end{array}$ & $\begin{array}{l}--- \\
---\end{array}$ & $\begin{array}{l}--- \\
---\end{array}$ & $\begin{array}{l}--- \\
---\end{array}$ & $\begin{array}{l}--- \\
---\end{array}$ & $\begin{array}{l}--- \\
---\end{array}$ & $\begin{array}{l}--- \\
---\end{array}$ & $\begin{array}{l}31800 \\
31900\end{array}$ & $\begin{array}{l}10200 \\
11700\end{array}$ & $\begin{array}{l}26600 \\
24800\end{array}$ & $\begin{array}{l}--- \\
---\end{array}$ & $\begin{array}{l}--- \\
---\end{array}$ & $\begin{array}{l}--- \\
---\end{array}$ \\
\hline 6 & --- & --- & --- & --- & --- & --- & 25900 & 6420 & 15500 & --- & --- & - \\
\hline 7 & --- & --- & --- & --- & --- & --- & 8330 & 2600 & 4420 & --- & --- & - \\
\hline 8 & --- & --- & --- & --- & --- & --- & 3390 & 2090 & 2500 & 14700 & 12400 & 13700 \\
\hline 9 & $\begin{array}{l}--- \\
---\end{array}$ & --- & --- & --- & --- & --- & 5710 & 2070 & 3890 & 14600 & 11400 & 13200 \\
\hline 10 & --- & --- & --- & --- & --- & --- & --- & --- & --- & 14200 & 12200 & 13100 \\
\hline 11 & --- & --- & --- & --- & --- & --- & --- & --- & --- & 13500 & 11000 & 12300 \\
\hline 12 & --- & --- & --- & --- & --- & --- & 2830 & 2080 & 2270 & 11400 & 10400 & 10900 \\
\hline 13 & --- & --- & --- & --- & --- & --- & 3600 & 2190 & 2540 & 10800 & 9970 & 10400 \\
\hline $\begin{array}{l}14 \\
15\end{array}$ & $\begin{array}{l}--- \\
---\end{array}$ & $\begin{array}{l}--- \\
---\end{array}$ & $\begin{array}{l}--- \\
---\end{array}$ & $\begin{array}{l}472 \\
606\end{array}$ & $\begin{array}{l}301 \\
344\end{array}$ & $\begin{array}{l}361 \\
449\end{array}$ & 4070 & 2760 & 3100 & 13700 & 9660 & 10600 \\
\hline & & & & & & & & & & & & \\
\hline $\begin{array}{l}16 \\
17\end{array}$ & $\begin{array}{l}--- \\
---\end{array}$ & $\begin{array}{l}--- \\
---\end{array}$ & $\begin{array}{l}--- \\
---\end{array}$ & $\begin{array}{l}658 \\
640\end{array}$ & $\begin{array}{l}341 \\
310\end{array}$ & $\begin{array}{l}481 \\
467\end{array}$ & $\begin{array}{l}3970 \\
3560\end{array}$ & $\begin{array}{l}2650 \\
1210\end{array}$ & $\begin{array}{l}2930 \\
2600\end{array}$ & $\begin{array}{l}14300 \\
14800\end{array}$ & $\begin{array}{r}9490 \\
11100\end{array}$ & $\begin{array}{l}11800 \\
13100\end{array}$ \\
\hline 18 & 110 & 30 & 52 & 390 & 278 & 313 & 2100 & $\begin{array}{r}1210 \\
811\end{array}$ & $\begin{array}{l}2600 \\
1110\end{array}$ & $\begin{array}{l}14800 \\
12800\end{array}$ & $\begin{array}{l}11100 \\
11900\end{array}$ & 12300 \\
\hline 19 & 158 & 26 & 72 & 417 & 243 & 306 & 1280 & 702 & 857 & 13200 & 12200 & 12800 \\
\hline 20 & 166 & 18 & 77 & 359 & 270 & 304 & 829 & 628 & 719 & 12700 & 11800 & 12300 \\
\hline 21 & 135 & 16 & 61 & 522 & 305 & 391 & 811 & 606 & 705 & --- & --- & \\
\hline 22 & 106 & 24 & 44 & 572 & 337 & 453 & 734 & 553 & 668 & --- & --- & \\
\hline 23 & 127 & 34 & 59 & 339 & 250 & 295 & 800 & 526 & 626 & --- & --- & --- \\
\hline $\begin{array}{l}24 \\
25\end{array}$ & $\begin{array}{l}129 \\
182\end{array}$ & $\begin{array}{l}26 \\
27\end{array}$ & $\begin{array}{l}61 \\
96\end{array}$ & $\begin{array}{l}338 \\
523\end{array}$ & $\begin{array}{l}243 \\
272\end{array}$ & $\begin{array}{l}290 \\
376\end{array}$ & $\begin{array}{l}835 \\
807\end{array}$ & $\begin{array}{l}664 \\
626\end{array}$ & $\begin{array}{l}726 \\
674\end{array}$ & $\begin{array}{l}--- \\
---\end{array}$ & $\begin{array}{l}--- \\
---\end{array}$ & $\begin{array}{l}--- \\
---\end{array}$ \\
\hline 26 & 164 & 29 & 89 & 487 & 235 & 324 & --- & --- & --- & --- & --- & \\
\hline 27 & 141 & 24 & 76 & 316 & 230 & 253 & --- & --- & --- & --- & --- & --- \\
\hline 28 & 160 & 24 & 78 & 543 & 269 & 326 & --- & --- & --- & --- & --- & --- \\
\hline 29 & 158 & 27 & 78 & 4490 & 384 & 643 & --- & --- & --- & 30100 & 19500 & 25700 \\
\hline 30 & 226 & 19 & 58 & 20900 & 832 & 2510 & --- & --- & --- & 35400 & 25700 & 30600 \\
\hline 31 & 143 & 29 & & -- & --- & --- & --- & --- & --- & 36700 & 25500 & \\
\hline MONTH & 226 & 16 & 70 & 20900 & 98 & 463 & 33300 & 526 & 6620 & 36700 & 9490 & 15300 \\
\hline \multirow[t]{2}{*}{ DAY } & MAX & MIN & MEAN & MAX & MIN & MEAN & MAX & MIN & MEAN & & & \\
\hline & \multicolumn{3}{|c|}{ SEPTEMBER } & \multicolumn{3}{|c|}{ OCTOBER } & \multicolumn{3}{|c|}{ NOVEMBER } & & & \\
\hline 1 & 35000 & 24900 & 30400 & 25000 & 23000 & 24100 & 831 & 323 & 492 & & & \\
\hline 2 & 28800 & 17800 & 22600 & 23200 & 19900 & 21400 & 323 & 203 & 247 & & & \\
\hline 3 & 22500 & 17800 & 19800 & 21000 & 17000 & 18900 & 203 & 176 & 186 & & & \\
\hline 4 & 21000 & 13500 & 17000 & 19400 & 17100 & 17900 & 195 & 174 & 182 & & & \\
\hline 5 & 16100 & 14700 & 15300 & 17400 & 15900 & 16500 & 221 & 190 & 198 & & & \\
\hline 6 & 17000 & 15700 & 16300 & 16200 & 11500 & 13500 & 225 & 208 & 216 & & & \\
\hline 7 & 18600 & 16300 & 17300 & 11500 & 7280 & 9310 & 208 & 200 & 204 & & & \\
\hline 8 & 19400 & 16700 & 18100 & 8690 & 5520 & 6890 & 203 & 186 & 194 & & & \\
\hline 9 & 19400 & 17800 & 18800 & 5520 & 3490 & 4240 & 315 & 188 & 221 & & & \\
\hline 10 & 19900 & 18400 & 19300 & 3980 & 2140 & 3270 & 278 & 139 & 170 & & & \\
\hline 11 & 20200 & 18700 & 19700 & 2210 & 1140 & 1680 & 193 & 136 & 156 & & & \\
\hline 12 & 19900 & 19400 & 19700 & 1480 & 988 & 1170 & 284 & 151 & 204 & & & \\
\hline 13 & 20800 & 19400 & 20200 & 1600 & 1120 & 1240 & 383 & 181 & 256 & & & \\
\hline 14 & 21100 & 19000 & 20400 & 4180 & 1160 & 2010 & 964 & 198 & $\begin{array}{l}370 \\
336\end{array}$ & & & \\
\hline 15 & 24100 & 20300 & 22500 & 3880 & 1820 & 2440 & 736 & 216 & 336 & & & \\
\hline 16 & 25800 & 21400 & 23700 & 4130 & 1690 & 2380 & 217 & 139 & 164 & & & \\
\hline 17 & 23200 & 20600 & 21200 & 4220 & 1870 & 2490 & 157 & 130 & 144 & & & \\
\hline 18 & 20800 & 19400 & 20000 & 5530 & 2010 & 3040 & 162 & 136 & 150 & & & \\
\hline 19 & 19500 & 17200 & 18200 & 2940 & 2100 & 2460 & 157 & 144 & 151 & & & \\
\hline 20 & 19800 & 15700 & 17300 & 2100 & 1110 & 1470 & 171 & 145 & 154 & & & \\
\hline 21 & 20400 & 16100 & 17700 & 1190 & 852 & 994 & 170 & 143 & 159 & & & \\
\hline 22 & 20000 & 17600 & 18400 & 1000 & 731 & 843 & 143 & 122 & 132 & & & \\
\hline 23 & 20600 & 17200 & 17900 & 829 & 749 & 786 & 122 & 116 & 118 & & & \\
\hline 24 & 20500 & 17300 & 18900 & 889 & 800 & 835 & 122 & 115 & 118 & & & \\
\hline 25 & 22800 & 16200 & 18800 & 2450 & 877 & 1230 & 132 & 119 & 126 & & & \\
\hline 26 & 26300 & 17500 & 21900 & 4000 & 1350 & 2200 & 253 & 126 & 141 & & & \\
\hline 27 & 32500 & 23100 & 28400 & 5710 & 1520 & 2960 & 296 & 62 & 112 & & & \\
\hline 28 & 33500 & 28700 & 32100 & 8820 & 1970 & 4590 & --- & --- & --- & & & \\
\hline 29 & 31700 & 26400 & 28800 & 6150 & 2950 & 4180 & ---- & --- & --- & & & \\
\hline $\begin{array}{l}30 \\
31\end{array}$ & 28300 & 23300 & 25900 & $\begin{array}{l}4810 \\
2110\end{array}$ & $\begin{array}{r}1660 \\
586\end{array}$ & $\begin{array}{l}2790 \\
1040\end{array}$ & --- & --- & --- & & & \\
\hline MONTH & 35000 & 13500 & 20900 & 25000 & 586 & 5770 & 964 & 62 & 196 & & & \\
\hline
\end{tabular}


Table A18. Specific conductance and water temperature data collected May-November 2000 at station 104 (USGS identifier 442509068181901) Northeast Creek near Bar Harbor, ME - Continued.

DEEP

Specific conductance, water, microsiemens per centimeter at 25 degrees Celsius

\begin{tabular}{|c|c|c|c|c|c|c|c|c|c|c|c|c|}
\hline \multirow[t]{2}{*}{ DAY } & MAX & MIN & MEAN & MAX & MIN & MEAN & MAX & MIN & MEAN & MAX & MIN & MEAN \\
\hline & & MAY & & \multicolumn{3}{|c|}{ JUNE } & \multicolumn{3}{|c|}{ JULY } & \multicolumn{3}{|c|}{ AUGUST } \\
\hline 1 & --- & --- & --- & 242 & 95 & 125 & 26800 & 20200 & 23900 & --- & --- & \\
\hline 2 & --- & --- & --- & 426 & 122 & 176 & 31700 & 23100 & 28500 & --- & --- & \\
\hline 3 & --- & --- & --- & --- & --- & --- & 35200 & 27700 & 32900 & --- & --- & \\
\hline 4 & --- & -- & --- & --- & --- & --- & 35500 & 29200 & 31100 & --- & --- & \\
\hline 5 & --- & --- & --- & --- & --- & --- & 31400 & 27000 & 29100 & --- & --- & --- \\
\hline 6 & --- & --- & --- & --- & --- & --- & 27400 & 24000 & 25500 & --- & --- & \\
\hline 7 & --- & --- & --- & --- & --- & --- & 24600 & 23900 & 24200 & --- & & \\
\hline 8 & --- & --- & --- & --- & --- & --- & 24300 & 23500 & 23900 & 24700 & 17100 & 20600 \\
\hline 9 & --- & --- & --- & --- & --- & --- & 24200 & 22900 & 23600 & 21000 & 14700 & 15800 \\
\hline 10 & --- & --- & --- & --- & --- & --- & -- & -- & -- & 24600 & 14400 & 19200 \\
\hline 11 & --- & --- & --- & --- & --- & --- & --- & --- & --- & 21400 & 18400 & 19900 \\
\hline 12 & --- & --- & --- & --- & --- & --- & 12200 & 5520 & 8130 & 21100 & 15100 & \\
\hline 13 & --- & --- & --- & --- & --- & --- & 12800 & 6630 & 9240 & 20300 & 17600 & 18900 \\
\hline 14 & --- & -- & --- & 958 & 425 & 600 & 12600 & 4990 & 8370 & 19100 & 17400 & 18000 \\
\hline 15 & --- & --- & --- & 852 & 488 & 654 & 14500 & 5720 & 9010 & 19100 & 16600 & 17700 \\
\hline 16 & --- & --- & --- & 814 & 577 & 693 & 12900 & 2640 & 6560 & 20200 & 16800 & 19100 \\
\hline 17 & --- & --- & --- & 721 & 567 & 635 & 4150 & 2960 & 3610 & 19600 & 18400 & \\
\hline 18 & 169 & 84 & 96 & 585 & 402 & 503 & 6850 & 3630 & 5110 & 20400 & 18200 & 19300 \\
\hline 19 & 91 & 70 & 75 & 614 & 392 & 520 & 4140 & 1810 & 2790 & 19600 & 17400 & 18400 \\
\hline 20 & 98 & 69 & 73 & 567 & 388 & 469 & 3040 & 1170 & 1990 & 17700 & 15000 & 16900 \\
\hline 21 & 149 & 73 & 83 & 510 & 358 & 431 & 6880 & 909 & 2880 & --- & --- & \\
\hline ר) & 170 & 81 & 105 & 639 & 424 & 515 & 1680 & 834 & 1090 & --- & --- & \\
\hline 23 & 185 & 86 & 104 & 456 & 337 & 387 & 4320 & 726 & 1920 & --- & --- & \\
\hline 24 & 93 & 69 & 86 & 530 & 266 & 382 & 3100 & 927 & 1670 & --- & --- & \\
\hline 25 & 69 & 55 & 61 & 608 & 324 & 445 & 2020 & 853 & 1300 & --- & --- & \\
\hline 26 & 59 & 55 & 56 & 709 & 415 & 534 & --- & --- & --- & --- & --- & \\
\hline & 62 & 50 & 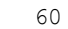 & 544 & 289 & 396 & --- & --- & --- & --- & --- & \\
\hline 28 & 69 & 62 & 66 & 493 & 298 & 383 & --- & --- & --- & --- & --- & \\
\hline 29 & 77 & 68 & 72 & 4550 & 384 & 687 & --- & --- & --- & 29400 & 25600 & 27900 \\
\hline 30 & 150 & 76 & 93 & 20200 & 3260 & 5510 & --- & --- & --- & 34400 & 27000 & 33000 \\
\hline 31 & 167 & 91 & 118 & & & & --- & --- & --- & 36400 & 30400 & 35100 \\
\hline MONTH & 185 & 55 & 82 & 20200 & 95 & 739 & 35500 & 726 & 13300 & 36400 & 14400 & 100 \\
\hline
\end{tabular}

\begin{tabular}{|c|c|c|c|c|c|c|c|c|c|}
\hline \multirow[t]{2}{*}{ DAY } & MAX & MIN & MEAN & MAX & MIN & MEAN & MAX & MIN & MEAN \\
\hline & \multicolumn{3}{|c|}{ SEPTEMBER } & \multicolumn{3}{|c|}{ OCTOBER } & \multicolumn{3}{|c|}{ NOVEMBER } \\
\hline $\begin{array}{l}1 \\
2 \\
3 \\
4 \\
5\end{array}$ & $\begin{array}{l}34500 \\
32500 \\
27900 \\
25500 \\
22900\end{array}$ & $\begin{array}{l}31100 \\
26500 \\
24400 \\
20200 \\
19600\end{array}$ & $\begin{array}{l}33200 \\
30600 \\
26200 \\
23100 \\
21200\end{array}$ & $\begin{array}{l}25300 \\
23800 \\
21900 \\
20400 \\
19500\end{array}$ & $\begin{array}{l}23400 \\
21600 \\
19700 \\
19100 \\
17000\end{array}$ & $\begin{array}{l}24300 \\
22600 \\
20900 \\
19800 \\
17900\end{array}$ & $\begin{array}{l}820 \\
321 \\
207 \\
192 \\
218\end{array}$ & $\begin{array}{l}321 \\
205 \\
173 \\
172 \\
187\end{array}$ & $\begin{array}{l}489 \\
246 \\
185 \\
180 \\
195\end{array}$ \\
\hline $\begin{array}{r}6 \\
7 \\
8 \\
9 \\
10\end{array}$ & $\begin{array}{l}22300 \\
23800 \\
24700 \\
23300 \\
21400\end{array}$ & $\begin{array}{l}19300 \\
21400 \\
22500 \\
21000 \\
18900\end{array}$ & $\begin{array}{l}20900 \\
22700 \\
23600 \\
22400 \\
19600\end{array}$ & $\begin{array}{l}17300 \\
13000 \\
13200 \\
11100 \\
10700\end{array}$ & $\begin{array}{r}12200 \\
12100 \\
11100 \\
5010 \\
4840\end{array}$ & $\begin{array}{r}14700 \\
12400 \\
12000 \\
8230 \\
6730\end{array}$ & $\begin{array}{l}222 \\
291 \\
200 \\
311 \\
285\end{array}$ & $\begin{array}{l}204 \\
196 \\
183 \\
185 \\
137\end{array}$ & $\begin{array}{l}213 \\
213 \\
191 \\
220 \\
174\end{array}$ \\
\hline $\begin{array}{l}11 \\
12 \\
13 \\
14 \\
15\end{array}$ & $\begin{array}{l}19600 \\
20000 \\
20600 \\
20500 \\
24100\end{array}$ & $\begin{array}{l}19000 \\
19200 \\
19700 \\
19800 \\
19800\end{array}$ & $\begin{array}{l}19300 \\
19400 \\
20300 \\
20100 \\
22400\end{array}$ & $\begin{array}{l}9360 \\
3980 \\
4850 \\
4270 \\
4000\end{array}$ & $\begin{array}{l}2780 \\
2280 \\
1620 \\
1830 \\
2110\end{array}$ & $\begin{array}{l}6120 \\
2920 \\
2890 \\
2970 \\
2890\end{array}$ & $\begin{array}{r}192 \\
275 \\
394 \\
1020 \\
861\end{array}$ & $\begin{array}{l}133 \\
148 \\
178 \\
198 \\
226\end{array}$ & $\begin{array}{l}153 \\
201 \\
256 \\
422 \\
368\end{array}$ \\
\hline $\begin{array}{l}16 \\
17 \\
18 \\
19 \\
20\end{array}$ & $\begin{array}{l}25400 \\
23500 \\
20600 \\
19600 \\
19200\end{array}$ & $\begin{array}{l}22700 \\
20300 \\
19100 \\
18300 \\
17100\end{array}$ & $\begin{array}{l}23900 \\
21600 \\
19800 \\
18800 \\
17900\end{array}$ & $\begin{array}{l}4620 \\
4330 \\
5840 \\
5530 \\
2240\end{array}$ & $\begin{array}{l}1830 \\
2750 \\
2480 \\
2130 \\
1530\end{array}$ & $\begin{array}{l}2860 \\
3470 \\
3890 \\
3520 \\
1820\end{array}$ & $\begin{array}{l}264 \\
171 \\
193 \\
164 \\
238\end{array}$ & $\begin{array}{l}142 \\
132 \\
136 \\
142 \\
151\end{array}$ & $\begin{array}{l}171 \\
146 \\
152 \\
150 \\
201\end{array}$ \\
\hline $\begin{array}{l}21 \\
22 \\
23 \\
24 \\
25\end{array}$ & $\begin{array}{l}20000 \\
19800 \\
20300 \\
20400 \\
22500\end{array}$ & $\begin{array}{l}17200 \\
17300 \\
17000 \\
17500 \\
17800\end{array}$ & $\begin{array}{l}18400 \\
18200 \\
17600 \\
19000 \\
19300\end{array}$ & $\begin{array}{l}2150 \\
1380 \\
1240 \\
1730 \\
2730\end{array}$ & $\begin{array}{r}1340 \\
918 \\
813 \\
943 \\
1060\end{array}$ & $\begin{array}{r}1600 \\
1130 \\
918 \\
1240 \\
1530\end{array}$ & $\begin{array}{l}393 \\
643 \\
624 \\
518 \\
485\end{array}$ & $\begin{array}{l}159 \\
382 \\
306 \\
314 \\
315\end{array}$ & $\begin{array}{l}278 \\
495 \\
409 \\
487 \\
360\end{array}$ \\
\hline $\begin{array}{l}26 \\
27 \\
28 \\
29 \\
30 \\
31\end{array}$ & $\begin{array}{r}25900 \\
32200 \\
33200 \\
32600 \\
28700 \\
---\end{array}$ & $\begin{array}{l}21500 \\
25900 \\
28600 \\
27800 \\
24900\end{array}$ & $\begin{array}{l}22900 \\
29400 \\
32500 \\
30100 \\
27200\end{array}$ & $\begin{array}{l}4020 \\
5510 \\
8650 \\
8360 \\
5340 \\
2100\end{array}$ & $\begin{array}{r}1730 \\
1640 \\
2740 \\
4380 \\
2100 \\
579\end{array}$ & $\begin{array}{l}2700 \\
3340 \\
5300 \\
5580 \\
3720 \\
1080\end{array}$ & $\begin{array}{l}459 \\
493 \\
--- \\
--- \\
--- \\
---\end{array}$ & $\begin{array}{r}331 \\
60 \\
--- \\
--- \\
--- \\
---\end{array}$ & $\begin{array}{l}361 \\
192 \\
--- \\
--- \\
--- \\
---\end{array}$ \\
\hline MONTH & 34500 & 17000 & 22700 & 25300 & 579 & 7130 & 1020 & 60 & 263 \\
\hline
\end{tabular}


Table A18. Specific conductance and water temperature data collected May-November 2000 at station 104 (USGS identifier 442509068181901) Northeast Creek near Bar Harbor, ME - Continued.

\begin{tabular}{|c|c|c|c|c|c|c|c|c|c|c|c|c|}
\hline \multirow[b]{2}{*}{ DAY } & \multicolumn{12}{|c|}{ Temperature, water, degrees Celsius } \\
\hline & MAX & MIN & MEAN & MAX & MIN & MEAN & MAX & MIN & MEAN & MAX & MIN & MEAN \\
\hline & & MAY & & \multicolumn{3}{|c|}{ JUNE } & \multicolumn{3}{|c|}{ JULY } & \multicolumn{3}{|c|}{ AUGUST } \\
\hline 1 & --- & --- & --- & 21.9 & 16.0 & 18.8 & 27.6 & 18.9 & 22.7 & --- & --- & --- \\
\hline 2 & --- & --- & --- & 20.9 & 18.3 & 19.7 & 29.5 & 21.2 & 24.3 & --- & --- & --- \\
\hline 3 & --- & --- & --- & --- & --- & --- & 25.4 & 23.0 & 23.9 & --- & --- & --- \\
\hline 4 & --- & --- & --- & --- & --- & --- & 27.1 & 22.6 & 24.3 & --- & --- & --- \\
\hline 5 & --- & --- & --- & --- & --- & --- & 26.2 & 21.2 & 24.3 & --- & --- & --- \\
\hline 6 & --- & --- & --- & --- & --- & --- & 26.2 & 20.5 & 23.6 & --- & --- & --- \\
\hline 7 & --- & --- & --- & --- & --- & --- & 26.3 & 19.9 & 22.8 & --- & --- & --- \\
\hline 8 & --- & --- & --- & --- & --- & --- & 22.6 & 19.2 & 20.8 & 29.5 & 21.6 & 25.2 \\
\hline 9 & --- & --- & --- & --- & --- & --- & 25.4 & 16.3 & 20.8 & 27.1 & 22.3 & 24.7 \\
\hline 10 & --- & --- & --- & --- & --- & --- & --- & --- & -- & 28.5 & 21.6 & 24.7 \\
\hline 11 & --- & --- & --- & --- & --- & --- & --- & --- & --- & 29.0 & 20.8 & 24.5 \\
\hline 12 & --- & --- & --- & --- & --- & --- & 27.6 & 19.2 & 23.4 & 23.0 & 19.5 & 21.5 \\
\hline 13 & --- & --- & --- & --- & --- & --- & 28.5 & 20.2 & 24.2 & 24.6 & 20.2 & 21.8 \\
\hline 14 & & --- & --- & 21.2 & 15.2 & 18.3 & 28.5 & 21.6 & 24.9 & 22.6 & 19.2 & 21.3 \\
\hline 15 & --- & --- & --- & 18.0 & 16.6 & 17.3 & 29.5 & 21.2 & 24.9 & 25.0 & 20.2 & 22.5 \\
\hline 16 & --- & --- & --- & 23.8 & 16.0 & 19.2 & 23.4 & 18.3 & 21.2 & 25.0 & 20.9 & 22.6 \\
\hline 17 & --- & --- & --- & 26.7 & 19.2 & 22.8 & 22.7 & 17.1 & 19.4 & 25.4 & 20.5 & 22.9 \\
\hline 18 & --- & --- & --- & 26.2 & 21.2 & 22.9 & 21.6 & 19.2 & 20.0 & 25.0 & 17.7 & 21.2 \\
\hline 19 & --- & --- & --- & 26.7 & 20.9 & 23.0 & 23.8 & 18.6 & 21.0 & 26.2 & 19.2 & 22.1 \\
\hline 20 & --- & --- & --- & 28.5 & 19.9 & 23.5 & 26.2 & 18.9 & 21.8 & 23.4 & 18.3 & 20.2 \\
\hline 21 & --- & --- & --- & 21.9 & 19.2 & 20.6 & 25.4 & 18.6 & 22.0 & --- & --- & --- \\
\hline 22 & --- & --- & --- & 25.8 & 18.0 & 21.3 & 21.9 & 19.9 & 20.7 & --- & --- & --- \\
\hline 23 & --- & --- & --- & 27.6 & 20.5 & 23.6 & 26.2 & 18.9 & 21.9 & --- & --- & $\begin{array}{l}--- \\
---\end{array}$ \\
\hline $\begin{array}{l}24 \\
25\end{array}$ & $\begin{array}{l}--- \\
---\end{array}$ & $\begin{array}{l}--- \\
---\end{array}$ & $\begin{array}{l}--- \\
---\end{array}$ & $\begin{array}{l}26.2 \\
24.6\end{array}$ & $\begin{array}{l}19.9 \\
19.2\end{array}$ & $\begin{array}{l}22.8 \\
22\end{array}$ & $\begin{array}{l}27.6 \\
29.5\end{array}$ & $\begin{array}{l}19.5 \\
20.5\end{array}$ & $\begin{array}{l}23.4 \\
24.8\end{array}$ & $\begin{array}{l}--- \\
---\end{array}$ & $\begin{array}{l}--- \\
---\end{array}$ & $\begin{array}{l}--- \\
---\end{array}$ \\
\hline 26 & --- & --- & --_ & 300 & 20.5 & 250 & --- & --- & --- & --- & --- & --- \\
\hline 27 & --- & --- & --- & 28.5 & 22.3 & 24.5 & --- & --- & --- & --- & --- & --- \\
\hline 28 & --- & --- & --- & 27.6 & 21.6 & 24.6 & --- & --- & --- & --- & --- & --- \\
\hline 29 & --- & --- & --- & 26.7 & 22.3 & 24.3 & --- & --- & --- & 26.2 & 18.3 & 23.0 \\
\hline 30 & --- & --- & --- & 23.0 & 20.5 & 21.7 & --- & --- & --- & 23.4 & 21.6 & 22.6 \\
\hline 31 & --- & --- & --- & -- & -- & -- & --- & --- & --- & 27.1 & 21.2 & 24.0 \\
\hline MONTH & --- & --- & --- & 30.0 & 15.2 & 21.9 & 29.5 & 16.3 & 22.7 & 29.5 & 17.7 & 22.8 \\
\hline \multirow{2}{*}{ DAY } & MAX & MIN & MEAN & MAX & MIN & MEAN & $\operatorname{MAX}$ & MIN & MEAN & & & \\
\hline & \multicolumn{3}{|c|}{ SEPTEMBER } & \multicolumn{3}{|c|}{ OCTOBER } & & VEMBEF & & & & \\
\hline 1 & 29.0 & 23.4 & 25.9 & 18.0 & 12.1 & 15.0 & 6.7 & 6.0 & 6.4 & & & \\
\hline 2 & 25.8 & 18.0 & 21.1 & 20.2 & 14.2 & 17.2 & 8.0 & 6.4 & 7.3 & & & \\
\hline 3 & 21.2 & 17.4 & 19.0 & 20.9 & 14.0 & 17.1 & 9.0 & 6.6 & 7.7 & & & \\
\hline 4 & 20.2 & 15.5 & 18.4 & 18.0 & 14.2 & 16.3 & 7.1 & 6.0 & 6.5 & & & \\
\hline 5 & 21.6 & 12.8 & 16.8 & 17.7 & 11.4 & 14.6 & 6.7 & 6.0 & 6.4 & & & \\
\hline 6 & 22.6 & 12.5 & 17.1 & 13.7 & 11.0 & 11.8 & 7.1 & 6.2 & 6.7 & & & \\
\hline 7 & 20.9 & 12.5 & 16.6 & 14.7 & 10.6 & 12.5 & 8.6 & 6.7 & 7.6 & & & \\
\hline 8 & 24.6 & 14.2 & 18.8 & 14.5 & 8.4 & 11.3 & 9.6 & 7.8 & 8.5 & & & \\
\hline 9 & 26.7 & 15.5 & 20.9 & 9.7 & 6.7 & 7.8 & 10.8 & 8.2 & 9.0 & & & \\
\hline 10 & 28.1 & 15.5 & 21.0 & 8.0 & 5.9 & 7.0 & 8.4 & 7.4 & 7.7 & & & \\
\hline 11 & 26.2 & 15.0 & 20.0 & 8.0 & 5.2 & 6.2 & 9.3 & 7.6 & 8.4 & & & \\
\hline 12 & 23.0 & 18.0 & 20.1 & 9.8 & 4.4 & 7.1 & 9.8 & 8.4 & 8.9 & & & \\
\hline 13 & 26.2 & 18.9 & 21.3 & 13.3 & 6.7 & 9.8 & 9.0 & 8.0 & 8.5 & & & \\
\hline 14 & 23.8 & 17.1 & 20.1 & 15.5 & 8.9 & 11.8 & 8.8 & 8.2 & 8.6 & & & \\
\hline 15 & 19.2 & 17.4 & 18.5 & 15.8 & 11.0 & 13.6 & 9.0 & 6.7 & 8.4 & & & \\
\hline 16 & 21.9 & 17.1 & 19.2 & 11.2 & 7.3 & 9.6 & 6.8 & 5.4 & 6.1 & & & \\
\hline 17 & 18.3 & 14.5 & 16.4 & 12.6 & 6.9 & 9.0 & 6.9 & 5.2 & 6.1 & & & \\
\hline 18 & 21.9 & 15.0 & 18.1 & 11.0 & 7.1 & 9.0 & 6.0 & 3.3 & 4.5 & & & \\
\hline 19 & 19.2 & 15.2 & 17.5 & 11.4 & 9.7 & 10.7 & 4.0 & 2.3 & 2.9 & & & \\
\hline 20 & 19.9 & 17.4 & 18.3 & 11.7 & 7.4 & 9.6 & 2.3 & 1.7 & 2.1 & & & \\
\hline 21 & 21.9 & 18.3 & 19.9 & 13.7 & 9.0 & 11.1 & 2.6 & 1.7 & 2.1 & & & \\
\hline 22 & 20.9 & 14.5 & 17.7 & 13.0 & 8.4 & 10.3 & 2.0 & 1.2 & 1.5 & & & \\
\hline 23 & 18.3 & 15.2 & 16.8 & 10.2 & 5.9 & 7.9 & 1.9 & 0.8 & 1.2 & & & \\
\hline 24 & 18.0 & 16.3 & 17.2 & 10.2 & 5.4 & 7.6 & 1.6 & 0.6 & 1.0 & & & \\
\hline 25 & 19.2 & 11.4 & 16.1 & 11.9 & 6.7 & 9.1 & 1.5 & 0.5 & 0.9 & & & \\
\hline 26 & & & 15.8 & 13.3 & 8.0 & 10.6 & 1.1 & 0.4 & 0.7 & & & \\
\hline 27 & 18.9 & 12.8 & 16.0 & 12.8 & 9.9 & 11.5 & 2.9 & 1.1 & 1.9 & & & \\
\hline 28 & 16.6 & 13.5 & 15.2 & 12.1 & 7.6 & 10.7 & --- & --- & --- & & & \\
\hline 29 & 16.3 & 11.2 & 13.8 & 8.4 & 3.9 & 5.9 & --- & --- & --- & & & \\
\hline 30 & 16.3 & 11.4 & 13.9 & 6.7 & 3.2 & 5.1 & --- & --- & --- & & & \\
\hline 31 & & & & 6.4 & 5. & 6.1 & --- & -1 & & & & \\
\hline MONTH & 29.0 & 11.2 & 18.2 & 20.9 & 3.2 & 10.4 & 10.8 & 0.4 & 5.5 & & & \\
\hline
\end{tabular}


Table A18. Specific conductance and water temperature data collected May-November 2000 at station 104 (USGS identifier 442509068181901) Northeast Creek near Bar Harbor, ME - Continued.

DEEP

Temperature, water, degrees Celsius

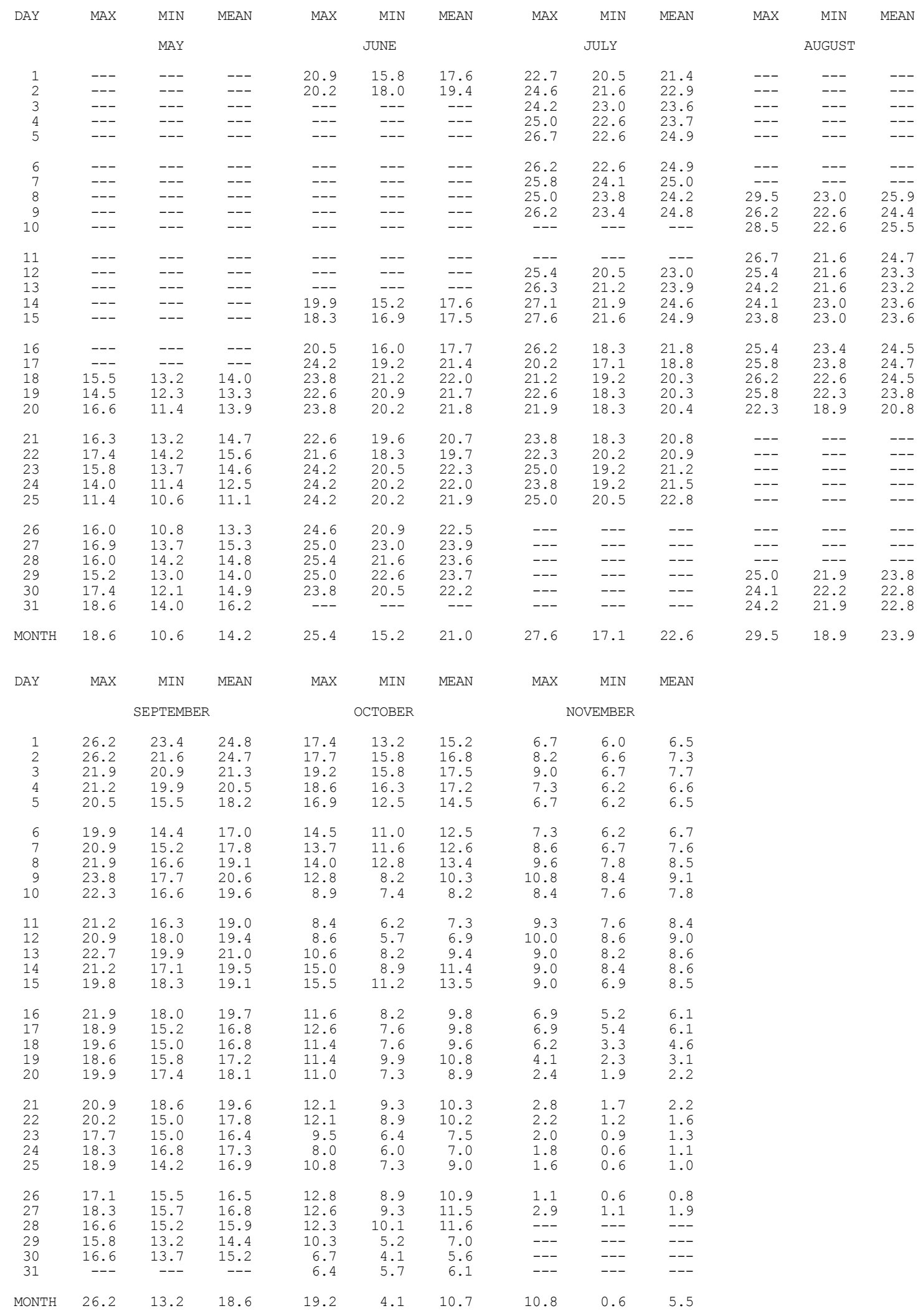


Table A19. Specific conductance and water temperature data collected May-November 2000 at station 105 (USGS identifier 442516068175501) Northeast Creek near Bar Harbor, ME [Data are presented as minimum, mean and maximum daily values of continuous data at 15-minute intervals. Shallow and deep data were collected at fixed depths located, respectively, about $0.2 \mathrm{~m}$ below the water surface and about $0.2 \mathrm{~m}$ above the stream bottom.]

SHALLOW

Specific conductance, water, microsiemens per centimeter at 25 degrees Celsius

\begin{tabular}{|c|c|c|c|c|c|c|c|c|c|c|c|c|}
\hline \multirow[t]{2}{*}{ DAY } & MAX & MIN & MEAN & MAX & MIN & MEAN & MAX & MIN & MEAN & MAX & MIN & MEAN \\
\hline & & MAY & & \multicolumn{3}{|c|}{ JUNE } & \multicolumn{3}{|c|}{ JULY } & \multicolumn{3}{|c|}{ AUGUST } \\
\hline $\begin{array}{l}1 \\
2 \\
3 \\
4 \\
5\end{array}$ & $\begin{array}{l}--- \\
--- \\
--- \\
--- \\
---\end{array}$ & $\begin{array}{l}--- \\
--- \\
--- \\
--- \\
---\end{array}$ & $\begin{array}{l}--- \\
--- \\
--- \\
--- \\
---\end{array}$ & $\begin{array}{r}70 \\
81 \\
104 \\
1010 \\
6820\end{array}$ & $\begin{array}{l}56 \\
58 \\
66 \\
64 \\
84\end{array}$ & $\begin{array}{r}59 \\
66 \\
77 \\
168 \\
4370\end{array}$ & $\begin{array}{r}3320 \\
14800 \\
24500 \\
29300 \\
28100\end{array}$ & $\begin{array}{r}147 \\
3280 \\
4610 \\
23500 \\
11600\end{array}$ & $\begin{array}{r}418 \\
12300 \\
22200 \\
27600 \\
20300\end{array}$ & $\begin{array}{l}21700 \\
27100 \\
27800 \\
22800 \\
15900\end{array}$ & $\begin{array}{r}5640 \\
12000 \\
12000 \\
10100 \\
9080\end{array}$ & $\begin{array}{l}16100 \\
24400 \\
23000 \\
14600 \\
12100\end{array}$ \\
\hline $\begin{array}{r}6 \\
7 \\
8 \\
9 \\
10\end{array}$ & $\begin{array}{l}--- \\
--- \\
--- \\
--- \\
---\end{array}$ & $\begin{array}{l}--- \\
--- \\
--- \\
--- \\
---\end{array}$ & $\begin{array}{l}--- \\
--- \\
--- \\
--- \\
---\end{array}$ & $\begin{array}{r}6810 \\
6810 \\
494 \\
280 \\
128\end{array}$ & $\begin{array}{r}850 \\
494 \\
137 \\
95 \\
76\end{array}$ & $\begin{array}{r}5980 \\
3210 \\
226 \\
156 \\
94\end{array}$ & $\begin{array}{r}23200 \\
3030 \\
1350 \\
1130 \\
---\end{array}$ & $\begin{array}{r}2910 \\
1020 \\
935 \\
333 \\
---\end{array}$ & $\begin{array}{r}12400 \\
1850 \\
1110 \\
600 \\
---\end{array}$ & $\begin{array}{r}11600 \\
11700 \\
3660 \\
3680 \\
6160\end{array}$ & $\begin{array}{l}5770 \\
1710 \\
1670 \\
1400 \\
2040\end{array}$ & $\begin{array}{l}8530 \\
6080 \\
2700 \\
2270 \\
3790\end{array}$ \\
\hline $\begin{array}{l}11 \\
12 \\
13 \\
14 \\
15\end{array}$ & $\begin{array}{l}--- \\
--- \\
--- \\
--- \\
---\end{array}$ & $\begin{array}{l}--- \\
--- \\
--- \\
--- \\
---\end{array}$ & $\begin{array}{l}--- \\
--- \\
--- \\
--- \\
---\end{array}$ & $\begin{array}{l}157 \\
147 \\
140 \\
157 \\
184\end{array}$ & $\begin{array}{r}100 \\
105 \\
77 \\
82 \\
97\end{array}$ & $\begin{array}{l}137 \\
125 \\
100 \\
115 \\
144\end{array}$ & $\begin{array}{l}--- \\
--- \\
--- \\
--- \\
---\end{array}$ & $\begin{array}{l}--- \\
--- \\
--- \\
--- \\
---\end{array}$ & $\begin{array}{l}--- \\
--- \\
--- \\
--- \\
---\end{array}$ & $\begin{array}{l}5750 \\
5680 \\
5420 \\
6140 \\
6130\end{array}$ & $\begin{array}{l}2720 \\
2600 \\
3250 \\
3120 \\
3010\end{array}$ & $\begin{array}{l}4440 \\
4880 \\
4130 \\
4430 \\
4410\end{array}$ \\
\hline $\begin{array}{l}16 \\
17 \\
18 \\
19 \\
20\end{array}$ & $\begin{array}{r}--- \\
--- \\
67 \\
69 \\
61\end{array}$ & $\begin{array}{r}--- \\
--- \\
63 \\
60 \\
59\end{array}$ & $\begin{array}{r}--- \\
--- \\
65 \\
64 \\
60\end{array}$ & $\begin{array}{r}152 \\
123 \\
96 \\
112 \\
117\end{array}$ & $\begin{array}{l}94 \\
71 \\
73 \\
67 \\
79\end{array}$ & $\begin{array}{r}121 \\
89 \\
81 \\
78 \\
100\end{array}$ & $\begin{array}{l}--- \\
--- \\
--- \\
--- \\
---\end{array}$ & $\begin{array}{l}--- \\
--- \\
--- \\
--- \\
---\end{array}$ & $\begin{array}{l}--- \\
--- \\
--- \\
--- \\
---\end{array}$ & $\begin{array}{r}10500 \\
9470 \\
9740 \\
10300 \\
9050\end{array}$ & $\begin{array}{l}3340 \\
4690 \\
5260 \\
3470 \\
3830\end{array}$ & $\begin{array}{l}7910 \\
7610 \\
6940 \\
6740 \\
6580\end{array}$ \\
\hline $\begin{array}{l}21 \\
22 \\
23 \\
24 \\
25\end{array}$ & $\begin{array}{l}63 \\
70 \\
69 \\
64 \\
59\end{array}$ & $\begin{array}{l}61 \\
62 \\
63 \\
55 \\
46\end{array}$ & $\begin{array}{l}61 \\
64 \\
66 \\
61 \\
50\end{array}$ & $\begin{array}{l}138 \\
120 \\
112 \\
112 \\
123\end{array}$ & $\begin{array}{l}95 \\
73 \\
67 \\
74 \\
90\end{array}$ & $\begin{array}{r}122 \\
98 \\
83 \\
93 \\
109\end{array}$ & $\begin{array}{l}--- \\
--- \\
--- \\
--- \\
---\end{array}$ & $\begin{array}{l}--- \\
--- \\
--- \\
--- \\
---\end{array}$ & $\begin{array}{l}--- \\
--- \\
--- \\
--- \\
---\end{array}$ & $\begin{array}{l}--- \\
--- \\
--- \\
--- \\
---\end{array}$ & $\begin{array}{l}--- \\
--- \\
--- \\
--- \\
---\end{array}$ & $\begin{array}{l}--- \\
--- \\
--- \\
--- \\
---\end{array}$ \\
\hline $\begin{array}{l}26 \\
27 \\
28 \\
29 \\
30 \\
31\end{array}$ & $\begin{array}{l}50 \\
50 \\
51 \\
52 \\
57 \\
63\end{array}$ & $\begin{array}{l}44 \\
46 \\
48 \\
50 \\
51 \\
53\end{array}$ & $\begin{array}{l}46 \\
48 \\
49 \\
51 \\
54 \\
58\end{array}$ & $\begin{array}{r}119 \\
105 \\
115 \\
422 \\
1030 \\
---\end{array}$ & $\begin{array}{r}76 \\
71 \\
71 \\
72 \\
65 \\
---\end{array}$ & $\begin{array}{r}95 \\
83 \\
94 \\
122 \\
217 \\
---\end{array}$ & $\begin{array}{r}--- \\
573 \\
1340 \\
3750 \\
6170 \\
15200\end{array}$ & $\begin{array}{r}--- \\
329 \\
297 \\
1010 \\
2340 \\
3740\end{array}$ & $\begin{array}{r}--- \\
397 \\
582 \\
1370 \\
4130 \\
9120\end{array}$ & $\begin{array}{r}--- \\
--- \\
--- \\
--- \\
19700 \\
20500\end{array}$ & $\begin{array}{r}--- \\
--- \\
--- \\
--- \\
13300 \\
12200\end{array}$ & $\begin{array}{r}--- \\
--- \\
--- \\
--- \\
17200 \\
18200\end{array}$ \\
\hline MONTH & 70 & 44 & 57 & 6820 & 56 & 554 & 29300 & 147 & 8170 & 27800 & 1400 & 9410 \\
\hline DAY & MAX & MIN & MEAN & MAX & MIN & MEAN & MAX & MIN & MEAN & & & \\
\hline & & SEPTEMB & & & CTOBER & & & VEMBER & & & & \\
\hline $\begin{array}{l}1 \\
2 \\
3 \\
4 \\
5\end{array}$ & $\begin{array}{l}19100 \\
18800 \\
18700 \\
17600 \\
13000\end{array}$ & $\begin{array}{l}13900 \\
13700 \\
15900 \\
12000 \\
11200\end{array}$ & $\begin{array}{l}16700 \\
16700 \\
17500 \\
14300 \\
12100\end{array}$ & $\begin{array}{l}12700 \\
11700 \\
11400 \\
11500 \\
11600\end{array}$ & $\begin{array}{l}10100 \\
10200 \\
10500 \\
10900 \\
11000\end{array}$ & $\begin{array}{l}11100 \\
10600 \\
10800 \\
11200 \\
11400\end{array}$ & $\begin{array}{r}150 \\
41 \\
52 \\
106 \\
134\end{array}$ & $\begin{array}{r}41 \\
36 \\
37 \\
52 \\
105\end{array}$ & $\begin{array}{r}76 \\
38 \\
43 \\
99 \\
120\end{array}$ & & & \\
\hline $\begin{array}{r}6 \\
7 \\
8 \\
9 \\
10\end{array}$ & $\begin{array}{l}13000 \\
13400 \\
13700 \\
14500 \\
14500\end{array}$ & $\begin{array}{l}11700 \\
12100 \\
12300 \\
11700 \\
11300\end{array}$ & $\begin{array}{l}12500 \\
12900 \\
13000 \\
13100 \\
12600\end{array}$ & $\begin{array}{r}11500 \\
7280 \\
2610 \\
3920 \\
2520\end{array}$ & $\begin{array}{r}7280 \\
2130 \\
1790 \\
1950 \\
780\end{array}$ & $\begin{array}{l}9970 \\
3420 \\
2190 \\
2510 \\
1340\end{array}$ & $\begin{array}{r}125 \\
129 \\
228 \\
142 \\
96\end{array}$ & $\begin{array}{r}114 \\
109 \\
108 \\
87 \\
82\end{array}$ & $\begin{array}{r}121 \\
118 \\
130 \\
112 \\
89\end{array}$ & & & \\
\hline $\begin{array}{l}11 \\
12 \\
13 \\
14 \\
15\end{array}$ & $\begin{array}{l}14600 \\
16000 \\
15800 \\
15500 \\
17600\end{array}$ & $\begin{array}{r}5740 \\
13800 \\
3440 \\
5480 \\
12300\end{array}$ & $\begin{array}{r}10600 \\
14900 \\
7500 \\
13800 \\
14700\end{array}$ & $\begin{array}{r}836 \\
815 \\
813 \\
984 \\
1190\end{array}$ & $\begin{array}{l}613 \\
661 \\
704 \\
792 \\
827\end{array}$ & $\begin{array}{l}713 \\
730 \\
748 \\
881 \\
957\end{array}$ & $\begin{array}{l}107 \\
132 \\
207 \\
299 \\
151\end{array}$ & $\begin{array}{r}87 \\
98 \\
104 \\
109 \\
104\end{array}$ & $\begin{array}{r}97 \\
115 \\
128 \\
150 \\
128\end{array}$ & & & \\
\hline $\begin{array}{l}16 \\
17 \\
18 \\
19 \\
20\end{array}$ & $\begin{array}{l}16600 \\
16600 \\
12000 \\
11900 \\
11200\end{array}$ & $\begin{array}{r}12900 \\
11400 \\
9830 \\
9600 \\
9570\end{array}$ & $\begin{array}{l}14900 \\
13500 \\
10600 \\
10300 \\
10100\end{array}$ & $\begin{array}{r}1400 \\
1480 \\
1770 \\
1610 \\
566\end{array}$ & $\begin{array}{r}1120 \\
1310 \\
1020 \\
566 \\
332\end{array}$ & $\begin{array}{r}1240 \\
1420 \\
1400 \\
1260 \\
409\end{array}$ & $\begin{array}{l}104 \\
157 \\
195 \\
270 \\
109\end{array}$ & $\begin{array}{l}84 \\
84 \\
85 \\
94 \\
98\end{array}$ & $\begin{array}{r}91 \\
99 \\
103 \\
117 \\
101\end{array}$ & & & \\
\hline $\begin{array}{l}21 \\
22 \\
23 \\
24 \\
25\end{array}$ & $\begin{array}{r}12500 \\
14200 \\
13000 \\
12600 \\
---\end{array}$ & $\begin{array}{r}9850 \\
11200 \\
10600 \\
9520 \\
---\end{array}$ & $\begin{array}{r}11100 \\
12000 \\
11300 \\
11100 \\
---\end{array}$ & $\begin{array}{l}405 \\
771 \\
735 \\
515 \\
511\end{array}$ & $\begin{array}{l}347 \\
377 \\
415 \\
269 \\
271\end{array}$ & $\begin{array}{l}384 \\
585 \\
568 \\
433 \\
385\end{array}$ & $\begin{array}{r}109 \\
93 \\
96 \\
248 \\
208\end{array}$ & $\begin{array}{l}93 \\
85 \\
85 \\
87 \\
95\end{array}$ & $\begin{array}{r}103 \\
87 \\
88 \\
123 \\
122\end{array}$ & & & \\
\hline $\begin{array}{l}26 \\
27 \\
28 \\
29 \\
30 \\
31\end{array}$ & $\begin{array}{r}17400 \\
20100 \\
21300 \\
21500 \\
15700 \\
----\end{array}$ & $\begin{array}{r}11000 \\
13100 \\
14300 \\
13700 \\
12000 \\
----\end{array}$ & $\begin{array}{r}13300 \\
17100 \\
17400 \\
16200 \\
13700 \\
---\end{array}$ & $\begin{array}{l}621 \\
680 \\
948 \\
959 \\
959 \\
524\end{array}$ & $\begin{array}{l}306 \\
416 \\
544 \\
771 \\
524 \\
150\end{array}$ & $\begin{array}{l}448 \\
508 \\
695 \\
834 \\
797 \\
320\end{array}$ & $\begin{array}{l}216 \\
104 \\
--- \\
--- \\
--- \\
---\end{array}$ & $\begin{array}{r}100 \\
58 \\
--- \\
--- \\
--- \\
---\end{array}$ & $\begin{array}{r}137 \\
65 \\
--- \\
--- \\
--- \\
---\end{array}$ & & & \\
\hline MONTH & 21500 & 3440 & 13300 & 12700 & 150 & 2910 & 299 & 36 & 104 & & & \\
\hline
\end{tabular}


Table A19. Specific conductance and water temperature data collected May-November 2000 at station 105 (USGS identifier 442516068175501) Northeast Creek near Bar Harbor, ME - Continued.

DEEP

Specific conductance, water, microsiemens per centimeter at 25 degrees Celsius

\begin{tabular}{|c|c|c|c|c|c|c|c|c|c|c|c|c|}
\hline \multirow[t]{2}{*}{ DAY } & MAX & MIN & MEAN & MAX & MIN & MEAN & MAX & MIN & MEAN & MAX & MIN & MEAN \\
\hline & & MAY & & \multicolumn{3}{|c|}{ JUNE } & \multicolumn{3}{|c|}{ JULY } & \multicolumn{3}{|c|}{ AUGUST } \\
\hline 1 & --- & --- & --- & --- & --- & --- & --- & --- & --- & 24900 & 18800 & 23100 \\
\hline 2 & --- & --- & --- & --- & --- & --- & --- & --- & --- & 28000 & 24200 & 26800 \\
\hline 3 & --- & --- & --- & --- & --- & --- & --- & --- & --- & 29800 & 27500 & 28700 \\
\hline 4 & --- & --- & --- & --- & --- & --- & --- & --- & --- & 29300 & 28200 & 28800 \\
\hline 5 & --- & --- & --- & --- & --- & --- & --- & --- & --- & 29400 & 27900 & 28500 \\
\hline 6 & --- & --- & --- & --- & --- & --- & --- & --- & --- & 28700 & 27600 & 28100 \\
\hline 7 & --- & --- & --- & --- & --- & --- & 29100 & 16300 & 21500 & 28200 & 27400 & 27900 \\
\hline 8 & --- & --- & --- & --- & --- & --- & 26100 & 22000 & 23700 & 27900 & 25400 & 26600 \\
\hline 9 & --- & --- & --- & --- & --- & --- & 23300 & 9900 & 19100 & 26500 & 22700 & 25300 \\
\hline 10 & --- & --- & --- & --- & --- & --- & -- & -- & -- & 25800 & 16100 & 21800 \\
\hline 11 & --- & --- & --- & --- & --- & --- & --- & --- & --- & 24000 & 18900 & 22000 \\
\hline 12 & --- & --- & --- & --- & --- & --- & --- & --- & --- & 25600 & 23300 & 24400 \\
\hline 13 & --- & --- & --- & --- & --- & --- & --- & --- & --- & 23300 & 19900 & 21200 \\
\hline 14 & --- & --- & --- & --- & --- & --- & --- & --- & --- & 25000 & 20100 & 23200 \\
\hline 15 & --- & --- & --- & --- & --- & --- & --- & --- & --- & 25200 & 20100 & 24400 \\
\hline 16 & --- & --- & --- & --- & --- & --- & --- & --- & --- & 23800 & 19200 & 21100 \\
\hline 17 & --- & --- & --- & --- & --- & --- & --- & --- & --- & 22500 & 15200 & 18800 \\
\hline 18 & --- & --- & --- & --- & --- & --- & --- & --- & --- & 21700 & 17000 & 19200 \\
\hline 19 & --- & --- & --- & --- & --- & --- & --- & --- & --- & 22300 & 16200 & 18900 \\
\hline 20 & --- & --- & --- & --- & --- & --- & --- & --- & --- & 21500 & 13000 & 17200 \\
\hline 21 & --- & --- & --- & --- & --- & --- & --- & --- & --- & --- & --- & \\
\hline 22 & --- & --- & --- & --- & --- & --- & --- & --- & --- & --- & --- & \\
\hline 23 & --- & --- & --- & --- & --- & --- & --- & --- & --- & --- & --- & --- \\
\hline 24 & --- & --- & --- & --- & --- & --- & --- & --- & --- & --- & --- & - \\
\hline 25 & --- & --- & --- & --- & --- & --- & --- & --- & --- & --- & --- & - \\
\hline 26 & --- & --- & --- & --- & --- & --- & --- & --- & --- & --- & --- & \\
\hline 27 & --- & --- & --- & --- & --- & --- & 890 & 188 & 456 & --- & --- & \\
\hline 28 & --- & --- & --- & --- & --- & --- & 4000 & 459 & 2360 & --- & --- & - \\
\hline 29 & --- & --- & --- & --- & --- & --- & 6580 & 3780 & 5210 & --- & --- & \\
\hline 30 & --- & --- & --- & --- & --- & --- & 9350 & 6580 & 7960 & 23600 & 18700 & 22000 \\
\hline 31 & --- & --- & --- & --- & --- & --- & 19900 & 7600 & 17400 & 26500 & 22700 & 25100 \\
\hline MONTH & --- & --- & --- & --- & --- & --- & 29100 & 188 & 12200 & 29800 & 13000 & 23800 \\
\hline
\end{tabular}

\begin{tabular}{|c|c|c|c|c|c|c|}
\hline \multirow[t]{2}{*}{ DAY } & MAX & MIN & MEAN & MAX & MIN & MEAN \\
\hline & \multicolumn{3}{|c|}{ SEPTEMBER } & \multicolumn{3}{|c|}{ OCTOBER } \\
\hline $\begin{array}{l}1 \\
2 \\
3 \\
4 \\
5\end{array}$ & $\begin{array}{l}28700 \\
29600 \\
29900 \\
29400 \\
29500\end{array}$ & $\begin{array}{l}25800 \\
25900 \\
27800 \\
27500 \\
24900\end{array}$ & $\begin{array}{l}27100 \\
28500 \\
28900 \\
28700 \\
27300\end{array}$ & $\begin{array}{l}27400 \\
25700 \\
25700 \\
23700 \\
21500\end{array}$ & $\begin{array}{l}24500 \\
23400 \\
23500 \\
21300 \\
20800\end{array}$ & $\begin{array}{l}25600 \\
24600 \\
24800 \\
22200 \\
21100\end{array}$ \\
\hline $\begin{array}{r}6 \\
7 \\
8 \\
9 \\
10\end{array}$ & $\begin{array}{l}29900 \\
29400 \\
28700 \\
26600 \\
25800\end{array}$ & $\begin{array}{l}25400 \\
26100 \\
24800 \\
23000 \\
23800\end{array}$ & $\begin{array}{l}27500 \\
28000 \\
26600 \\
25000 \\
24500\end{array}$ & $\begin{array}{r}22900 \\
15200 \\
8280 \\
4760 \\
3120\end{array}$ & $\begin{array}{r}12000 \\
6780 \\
4760 \\
2620 \\
754\end{array}$ & $\begin{array}{r}19800 \\
8980 \\
6300 \\
4080 \\
1460\end{array}$ \\
\hline $\begin{array}{l}11 \\
12 \\
13 \\
14 \\
15\end{array}$ & $\begin{array}{l}25600 \\
25500 \\
24700 \\
22400 \\
24100\end{array}$ & $\begin{array}{l}23300 \\
23800 \\
19900 \\
19400 \\
20000\end{array}$ & $\begin{array}{l}24300 \\
24600 \\
22300 \\
21200 \\
21700\end{array}$ & $\begin{array}{r}951 \\
1050 \\
1530 \\
1760 \\
1900\end{array}$ & $\begin{array}{l}590 \\
781 \\
800 \\
971 \\
800\end{array}$ & $\begin{array}{r}758 \\
899 \\
995 \\
1200 \\
1140\end{array}$ \\
\hline $\begin{array}{l}16 \\
17 \\
18 \\
19 \\
20\end{array}$ & $\begin{array}{l}22200 \\
21600 \\
21100 \\
20900 \\
21400\end{array}$ & $\begin{array}{l}20000 \\
20400 \\
20300 \\
20000 \\
20000\end{array}$ & $\begin{array}{l}21500 \\
21000 \\
20800 \\
20400 \\
20600\end{array}$ & $\begin{array}{r}2210 \\
2010 \\
2460 \\
1730 \\
828\end{array}$ & $\begin{array}{r}1140 \\
1420 \\
1100 \\
828 \\
278\end{array}$ & $\begin{array}{r}1420 \\
1560 \\
1570 \\
1360 \\
518\end{array}$ \\
\hline $\begin{array}{l}21 \\
22 \\
23 \\
24 \\
25\end{array}$ & $\begin{array}{r}21200 \\
20500 \\
18800 \\
19200 \\
---\end{array}$ & $\begin{array}{r}19700 \\
17200 \\
17100 \\
16000 \\
---\end{array}$ & $\begin{array}{r}20600 \\
19100 \\
17700 \\
17700 \\
---\end{array}$ & $\begin{array}{l}662 \\
838 \\
837 \\
776 \\
750\end{array}$ & $\begin{array}{l}246 \\
335 \\
453 \\
338 \\
250\end{array}$ & $\begin{array}{l}388 \\
590 \\
710 \\
589 \\
460\end{array}$ \\
\hline $\begin{array}{l}26 \\
27 \\
28 \\
29 \\
30 \\
31\end{array}$ & $\begin{array}{l}19900 \\
20800 \\
23300 \\
25100 \\
27900\end{array}$ & $\begin{array}{l}15600 \\
17900 \\
19100 \\
22900 \\
24700\end{array}$ & $\begin{array}{l}16700 \\
19300 \\
20900 \\
24500 \\
26500\end{array}$ & $\begin{array}{l}672 \\
697 \\
975 \\
883 \\
914 \\
396\end{array}$ & $\begin{array}{l}306 \\
276 \\
416 \\
667 \\
396\end{array}$ & $\begin{array}{l}440 \\
437 \\
610 \\
749 \\
783 \\
150\end{array}$ \\
\hline MONTH & 9900 & 15600 & 23200 & 27400 & 246 & 90 \\
\hline
\end{tabular}


Table A19. Specific conductance and water temperature data collected May-November 2000 at station 105 (USGS identifier 442516068175501) Northeast Creek near Bar Harbor, ME - Continued.

\begin{tabular}{|c|c|c|c|c|c|c|c|c|c|c|c|c|}
\hline \multirow[b]{2}{*}{ DAY } & \multicolumn{12}{|c|}{$\begin{array}{l}\text { SHALLOW } \\
\text { water, dec }\end{array}$} \\
\hline & MAX & MIN & MEAN & MAX & MIN & MEAN & MAX & MIN & MEAN & MAX & MIN & MEAN \\
\hline & & MAY & & & JUNE & & & JULY & & & AUGUST & \\
\hline $\begin{array}{l}1 \\
2 \\
3 \\
4 \\
5\end{array}$ & $\begin{array}{l}--- \\
--- \\
--- \\
--- \\
---\end{array}$ & $\begin{array}{l}--- \\
--- \\
--- \\
--- \\
---\end{array}$ & $\begin{array}{l}--- \\
--- \\
--- \\
--- \\
---\end{array}$ & $\begin{array}{l}22.6 \\
20.5 \\
20.2 \\
18.9 \\
20.2\end{array}$ & $\begin{array}{l}15.2 \\
16.8 \\
16.0 \\
15.7 \\
15.7\end{array}$ & $\begin{array}{l}18.3 \\
18.3 \\
17.9 \\
17.5 \\
18.1\end{array}$ & $\begin{array}{l}23.3 \\
24.5 \\
24.1 \\
25.3 \\
25.8\end{array}$ & $\begin{array}{l}17.7 \\
21.5 \\
23.0 \\
23.7 \\
23.7\end{array}$ & $\begin{array}{l}20.1 \\
22.8 \\
23.4 \\
24.2 \\
24.5\end{array}$ & $\begin{array}{l}27.1 \\
26.2 \\
25.8 \\
28.5 \\
29.5\end{array}$ & $\begin{array}{l}21.5 \\
23.7 \\
23.0 \\
23.0 \\
22.6\end{array}$ & $\begin{array}{l}24.2 \\
24.5 \\
24.4 \\
25.7 \\
25.8\end{array}$ \\
\hline $\begin{array}{r}6 \\
7 \\
8 \\
9 \\
10\end{array}$ & $\begin{array}{l}--- \\
--- \\
--- \\
--- \\
---\end{array}$ & $\begin{array}{l}--- \\
--- \\
--- \\
--- \\
---\end{array}$ & $\begin{array}{l}--- \\
--- \\
--- \\
--- \\
---\end{array}$ & $\begin{array}{l}19.5 \\
16.5 \\
17.7 \\
19.8 \\
18.6\end{array}$ & $\begin{array}{l}16.0 \\
13.4 \\
12.5 \\
14.4 \\
16.5\end{array}$ & $\begin{array}{l}17.9 \\
14.7 \\
14.9 \\
17.2 \\
17.2\end{array}$ & $\begin{array}{r}25.3 \\
24.5 \\
21.5 \\
24.9 \\
---\end{array}$ & $\begin{array}{r}20.8 \\
19.2 \\
17.1 \\
13.4 \\
---\end{array}$ & $\begin{array}{r}23.2 \\
21.3 \\
19.2 \\
19.2 \\
---\end{array}$ & $\begin{array}{l}29.5 \\
24.1 \\
30.5 \\
27.1 \\
28.5\end{array}$ & $\begin{array}{l}21.9 \\
19.8 \\
20.2 \\
17.1 \\
18.6\end{array}$ & $\begin{array}{l}25.3 \\
21.7 \\
24.4 \\
22.3 \\
23.6\end{array}$ \\
\hline $\begin{array}{l}11 \\
12 \\
13 \\
14 \\
15\end{array}$ & $\begin{array}{l}--- \\
--- \\
--- \\
--- \\
---\end{array}$ & $\begin{array}{l}--- \\
--- \\
--- \\
--- \\
---\end{array}$ & $\begin{array}{l}--- \\
--- \\
--- \\
--- \\
---\end{array}$ & $\begin{array}{l}16.5 \\
18.9 \\
18.3 \\
20.2 \\
16.8\end{array}$ & $\begin{array}{l}14.4 \\
13.7 \\
13.9 \\
14.4 \\
15.4\end{array}$ & $\begin{array}{l}15.2 \\
16.2 \\
16.1 \\
17.3 \\
16.2\end{array}$ & $\begin{array}{l}--- \\
--- \\
--- \\
--- \\
---\end{array}$ & $\begin{array}{l}--- \\
--- \\
--- \\
--- \\
---\end{array}$ & $\begin{array}{l}--- \\
--- \\
--- \\
--- \\
--\end{array}$ & $\begin{array}{l}28.0 \\
23.7 \\
24.5 \\
23.3 \\
24.9\end{array}$ & $\begin{array}{l}17.7 \\
16.0 \\
18.6 \\
18.9 \\
20.2\end{array}$ & $\begin{array}{l}23.0 \\
20.6 \\
21.1 \\
21.3 \\
22.2\end{array}$ \\
\hline $\begin{array}{l}16 \\
17 \\
18 \\
19 \\
20\end{array}$ & $\begin{array}{l}--- \\
--- \\
14.4 \\
13.4 \\
16.0\end{array}$ & $\begin{array}{r}--- \\
--- \\
13.0 \\
11.6 \\
10.9\end{array}$ & $\begin{array}{r}--- \\
--- \\
13.6 \\
12.8 \\
13.4\end{array}$ & $\begin{array}{l}23.0 \\
26.6 \\
24.5 \\
26.2 \\
24.5\end{array}$ & $\begin{array}{l}15.4 \\
18.6 \\
18.9 \\
18.6 \\
18.3\end{array}$ & $\begin{array}{l}18.2 \\
21.7 \\
21.3 \\
21.4 \\
21.2\end{array}$ & $\begin{array}{l}--- \\
--- \\
--- \\
--- \\
---\end{array}$ & $\begin{array}{l}--- \\
--- \\
--- \\
--- \\
---\end{array}$ & $\begin{array}{l}--- \\
--- \\
--- \\
--- \\
---\end{array}$ & $\begin{array}{l}24.5 \\
25.8 \\
26.2 \\
25.8 \\
23.7\end{array}$ & $\begin{array}{l}20.8 \\
19.2 \\
17.1 \\
18.9 \\
17.7\end{array}$ & $\begin{array}{l}22.3 \\
22.5 \\
21.5 \\
22.5 \\
20.1\end{array}$ \\
\hline $\begin{array}{l}21 \\
22 \\
23 \\
24 \\
25\end{array}$ & $\begin{array}{l}16.0 \\
17.4 \\
15.2 \\
13.2 \\
11.4\end{array}$ & $\begin{array}{l}13.4 \\
13.7 \\
13.2 \\
10.5 \\
10.1\end{array}$ & $\begin{array}{l}14.4 \\
15.2 \\
14.2 \\
12.0 \\
10.8\end{array}$ & $\begin{array}{l}21.5 \\
24.9 \\
24.9 \\
25.3 \\
24.1\end{array}$ & $\begin{array}{l}18.3 \\
17.4 \\
19.5 \\
18.9 \\
18.3\end{array}$ & $\begin{array}{l}19.5 \\
20.6 \\
22.3 \\
21.6 \\
21.4\end{array}$ & $\begin{array}{l}--- \\
--- \\
--- \\
--- \\
---\end{array}$ & $\begin{array}{l}--- \\
--- \\
--- \\
--- \\
---\end{array}$ & $\begin{array}{l}--- \\
--- \\
--- \\
--- \\
---\end{array}$ & $\begin{array}{l}--- \\
--- \\
--- \\
--- \\
---\end{array}$ & $\begin{array}{l}--- \\
--- \\
--- \\
--- \\
---\end{array}$ & $\begin{array}{l}--- \\
--- \\
--- \\
--- \\
---\end{array}$ \\
\hline $\begin{array}{l}26 \\
27 \\
28 \\
29 \\
30 \\
31\end{array}$ & $\begin{array}{l}16.0 \\
16.0 \\
15.2 \\
14.7 \\
17.7 \\
19.2\end{array}$ & $\begin{array}{l}10.5 \\
13.7 \\
13.9 \\
13.2 \\
12.0 \\
13.9\end{array}$ & $\begin{array}{l}12.9 \\
14.9 \\
14.4 \\
13.8 \\
14.7 \\
16.2\end{array}$ & $\begin{array}{l}27.5 \\
25.8 \\
29.5 \\
25.8 \\
23.3\end{array}$ & $\begin{array}{l}19.8 \\
20.8 \\
20.5 \\
21.5 \\
19.2\end{array}$ & $\begin{array}{l}23.0 \\
22.9 \\
24.1 \\
23.6 \\
20.8\end{array}$ & $\begin{array}{l}--- \\
22.2 \\
24.1 \\
27.5 \\
24.5 \\
25.8\end{array}$ & $\begin{array}{r}--- \\
19.2 \\
18.3 \\
20.5 \\
21.9 \\
21.2\end{array}$ & $\begin{array}{l}--- \\
20.0 \\
21.1 \\
23.9 \\
23.4 \\
23.8\end{array}$ & $\begin{array}{r}--- \\
--- \\
--- \\
--- \\
25.3 \\
27.5\end{array}$ & $\begin{array}{r}--- \\
--- \\
--- \\
--- \\
21.5 \\
21.2\end{array}$ & $\begin{array}{r}--- \\
--- \\
--- \\
--- \\
23.3 \\
24.4\end{array}$ \\
\hline MONTH & 19.2 & 10.1 & 13.8 & 29.5 & 12.5 & 19.2 & 27.5 & 13.4 & 22.1 & 30.5 & 16.0 & 23.0 \\
\hline \multirow[t]{2}{*}{ DAY } & MAX & MIN & MEAN & MAX & MIN & MEAN & MAX & MIN & MEAN & & & \\
\hline & \multicolumn{3}{|c|}{ SEPTEMBER } & \multicolumn{3}{|c|}{ OCTOBER } & \multicolumn{3}{|c|}{ NOVEMBER } & & & \\
\hline $\begin{array}{l}1 \\
2 \\
3 \\
4 \\
5\end{array}$ & $\begin{array}{l}29.5 \\
25.3 \\
21.5 \\
21.2 \\
20.8\end{array}$ & $\begin{array}{l}23.0 \\
19.8 \\
19.2 \\
16.5 \\
13.0\end{array}$ & $\begin{array}{l}25.8 \\
22.6 \\
20.2 \\
19.3 \\
16.8\end{array}$ & $\begin{array}{l}18.9 \\
19.8 \\
21.2 \\
17.7 \\
17.7\end{array}$ & $\begin{array}{l}11.4 \\
14.4 \\
13.9 \\
13.7 \\
11.8\end{array}$ & $\begin{array}{l}14.6 \\
16.6 \\
17.3 \\
15.8 \\
14.5\end{array}$ & $\begin{array}{l}6.7 \\
7.6 \\
8.0 \\
6.9 \\
6.7\end{array}$ & $\begin{array}{l}6.0 \\
6.4 \\
6.5 \\
5.8 \\
6.0\end{array}$ & $\begin{array}{l}6.3 \\
7.0 \\
7.2 \\
6.4 \\
6.3\end{array}$ & & & \\
\hline $\begin{array}{r}6 \\
7 \\
8 \\
9 \\
10\end{array}$ & $\begin{array}{l}22.2 \\
21.9 \\
24.1 \\
25.8 \\
25.3\end{array}$ & $\begin{array}{l}12.5 \\
13.7 \\
15.2 \\
15.2 \\
11.4\end{array}$ & $\begin{array}{l}17.1 \\
17.6 \\
19.7 \\
20.4 \\
18.1\end{array}$ & $\begin{array}{r}13.2 \\
13.7 \\
13.9 \\
10.9 \\
8.2\end{array}$ & $\begin{array}{r}11.4 \\
10.9 \\
8.9 \\
7.6 \\
6.7\end{array}$ & $\begin{array}{r}12.4 \\
12.3 \\
11.5 \\
8.8 \\
7.2\end{array}$ & $\begin{array}{l}6.9 \\
8.0 \\
8.9 \\
9.7 \\
8.2\end{array}$ & $\begin{array}{l}6.4 \\
6.7 \\
7.8 \\
8.0 \\
7.6\end{array}$ & $\begin{array}{l}6.7 \\
7.4 \\
8.2 \\
8.5 \\
7.9\end{array}$ & & & \\
\hline $\begin{array}{l}11 \\
12 \\
13 \\
14 \\
15\end{array}$ & $\begin{array}{l}23.3 \\
24.1 \\
24.9 \\
24.1 \\
19.5\end{array}$ & $\begin{array}{r}9.1 \\
17.7 \\
18.6 \\
16.0 \\
16.3\end{array}$ & $\begin{array}{l}17.7 \\
20.3 \\
21.2 \\
19.7 \\
17.8\end{array}$ & $\begin{array}{r}7.6 \\
11.0 \\
13.0 \\
14.2 \\
14.9\end{array}$ & $\begin{array}{r}5.8 \\
5.0 \\
6.9 \\
8.9 \\
11.4\end{array}$ & $\begin{array}{r}6.6 \\
7.8 \\
9.8 \\
11.6 \\
13.1\end{array}$ & $\begin{array}{l}8.9 \\
9.3 \\
8.7 \\
8.7 \\
8.9\end{array}$ & $\begin{array}{l}7.6 \\
8.2 \\
7.8 \\
8.2 \\
7.2\end{array}$ & $\begin{array}{l}8.3 \\
8.6 \\
8.3 \\
8.4 \\
8.4\end{array}$ & & & \\
\hline $\begin{array}{l}16 \\
17 \\
18 \\
19 \\
20\end{array}$ & $\begin{array}{l}21.9 \\
19.2 \\
23.0 \\
20.5 \\
20.5\end{array}$ & $\begin{array}{l}16.5 \\
15.2 \\
14.2 \\
15.2 \\
17.7\end{array}$ & $\begin{array}{l}19.1 \\
17.5 \\
18.0 \\
18.1 \\
19.1\end{array}$ & $\begin{array}{r}11.4 \\
12.1 \\
9.7 \\
10.5 \\
11.6\end{array}$ & $\begin{array}{l}8.5 \\
8.2 \\
7.8 \\
9.3 \\
7.8\end{array}$ & $\begin{array}{r}9.7 \\
9.5 \\
9.0 \\
10.1 \\
9.5\end{array}$ & $\begin{array}{l}7.2 \\
6.9 \\
6.0 \\
3.8 \\
2.4\end{array}$ & $\begin{array}{l}5.5 \\
5.3 \\
3.1 \\
2.0 \\
1.7\end{array}$ & $\begin{array}{l}6.1 \\
6.0 \\
4.7 \\
2.9 \\
2.0\end{array}$ & & & \\
\hline $\begin{array}{l}21 \\
22 \\
23 \\
24 \\
25\end{array}$ & $\begin{array}{r}23.3 \\
22.2 \\
17.7 \\
18.0 \\
---\end{array}$ & $\begin{array}{r}18.9 \\
16.5 \\
15.2 \\
16.0 \\
---\end{array}$ & $\begin{array}{r}20.7 \\
18.9 \\
16.5 \\
17.0 \\
---\end{array}$ & $\begin{array}{r}13.5 \\
11.4 \\
9.9 \\
11.0 \\
12.1\end{array}$ & $\begin{array}{l}8.3 \\
8.3 \\
6.2 \\
6.0 \\
6.5\end{array}$ & $\begin{array}{r}10.7 \\
9.8 \\
8.1 \\
8.1 \\
8.8\end{array}$ & $\begin{array}{l}2.6 \\
1.9 \\
2.0 \\
1.4 \\
1.2\end{array}$ & $\begin{array}{l}1.7 \\
1.0 \\
0.9 \\
0.5 \\
0.2\end{array}$ & $\begin{array}{l}2.0 \\
1.5 \\
1.4 \\
0.9 \\
0.7\end{array}$ & & & \\
\hline $\begin{array}{l}26 \\
27 \\
28 \\
29 \\
30 \\
31\end{array}$ & $\begin{array}{l}18.0 \\
20.2 \\
18.9 \\
16.0 \\
15.7\end{array}$ & $\begin{array}{r}14.2 \\
15.4 \\
12.0 \\
10.3 \\
9.1\end{array}$ & $\begin{array}{l}16.4 \\
18.1 \\
16.1 \\
13.4 \\
12.9\end{array}$ & $\begin{array}{r}12.3 \\
11.4 \\
10.5 \\
7.4 \\
6.0 \\
6.4\end{array}$ & $\begin{array}{l}7.2 \\
8.7 \\
7.4 \\
4.1 \\
2.9 \\
6.0\end{array}$ & $\begin{array}{r}9.5 \\
10.1 \\
9.6 \\
5.4 \\
4.7 \\
6.2\end{array}$ & $\begin{array}{l}1.4 \\
3.5 \\
--- \\
--- \\
--- \\
---\end{array}$ & $\begin{array}{l}0.5 \\
0.9 \\
--- \\
--- \\
--- \\
---\end{array}$ & $\begin{array}{l}0.9 \\
2.2 \\
--- \\
--- \\
--- \\
---\end{array}$ & & & \\
\hline MONTH & 29.5 & 9.1 & 18.5 & 21.2 & 2.9 & 10.3 & 9.7 & 0.2 & 5.4 & & & \\
\hline
\end{tabular}


Table A19. Specific conductance and water temperature data collected May-November 2000 at station 105 (USGS identifier 442516068175501) Northeast Creek near Bar Harbor, ME - Continued.

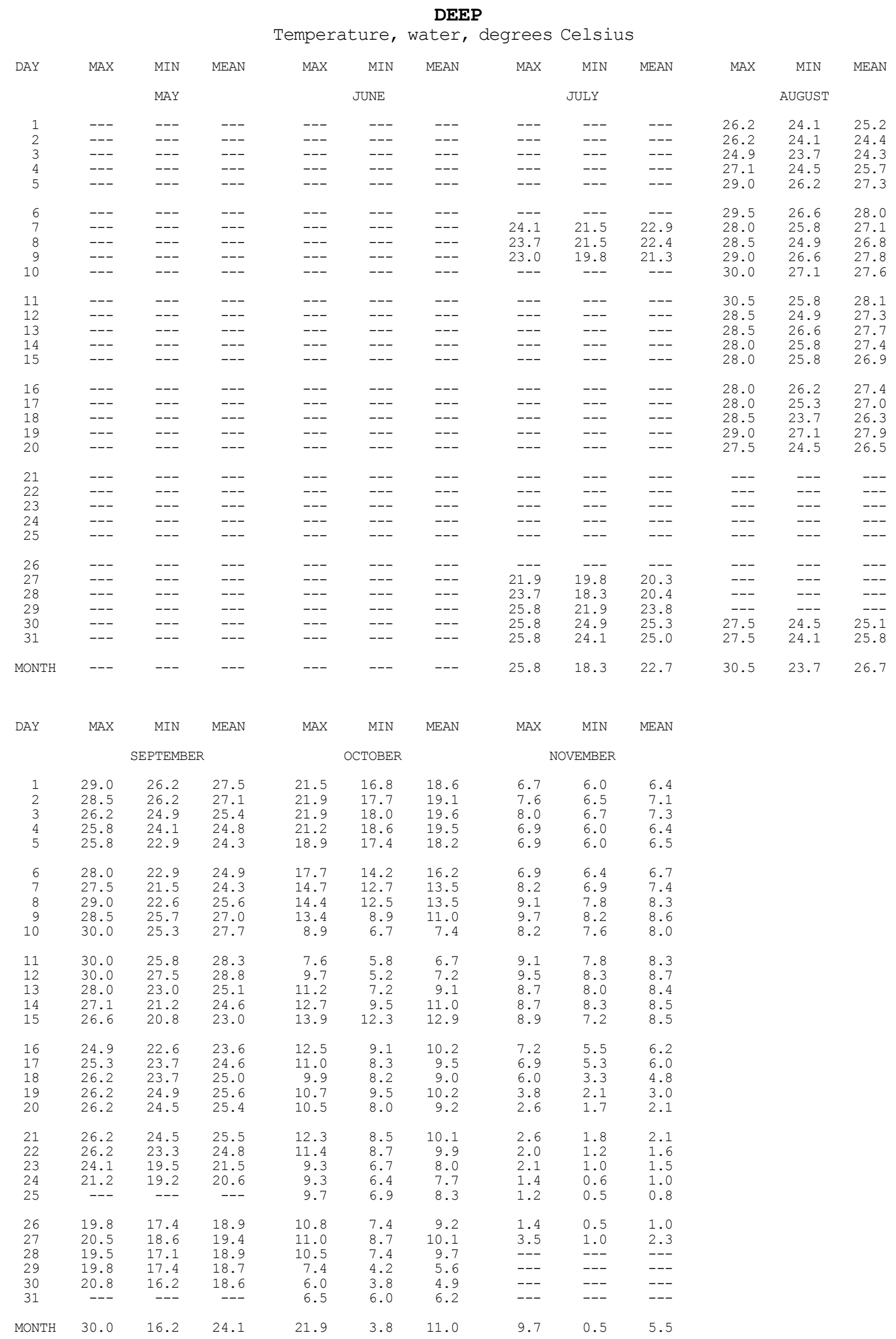


Table A20. Specific conductance and water temperature data collected May-November 2001 at station 101 (USGS identifier 01022820) Northeast Creek at Route 3 bridge near Bar Harbor, ME [Data are presented as minimum, mean and maximum daily values of continuous data at 15-minute intervals. Shallow and deep data were collected at fixed depths located, respectively, about $0.2 \mathrm{~m}$ below the water surface and about $0.2 \mathrm{~m}$ above the stream bottom.]

SHALLOW

Specific conductance, water, microsiemens per centimeter at 25 degrees Celsius

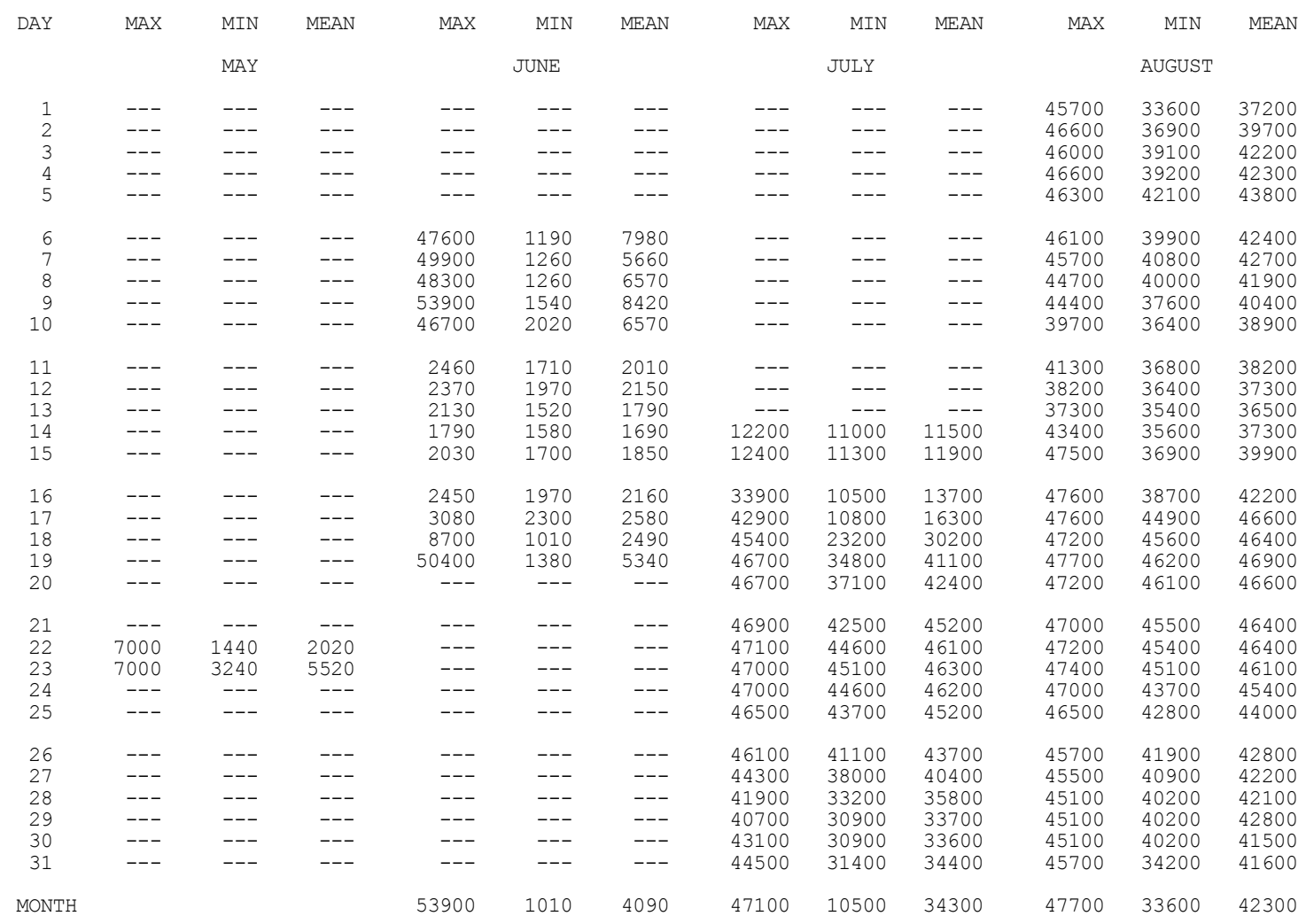

\begin{tabular}{|c|c|c|c|c|c|c|c|c|c|}
\hline \multirow[t]{2}{*}{ DAY } & MAX & MIN & MEAN & MAX & MIN & MEAN & MAX & MIN & MEAN \\
\hline & \multicolumn{3}{|c|}{ SEPTEMBER } & \multicolumn{3}{|c|}{ OCTOBER } & \multicolumn{3}{|c|}{ NOVEMBER } \\
\hline $\begin{array}{l}1 \\
2 \\
3 \\
4 \\
5\end{array}$ & $\begin{array}{l}44900 \\
43600 \\
45000 \\
44200 \\
45000\end{array}$ & $\begin{array}{l}40900 \\
38900 \\
38100 \\
40200 \\
42100\end{array}$ & $\begin{array}{l}43200 \\
41400 \\
40200 \\
41400 \\
43700\end{array}$ & $\begin{array}{l}44700 \\
44000 \\
43300 \\
44100 \\
43600\end{array}$ & $\begin{array}{l}33100 \\
36700 \\
37700 \\
39500 \\
39500\end{array}$ & $\begin{array}{l}35900 \\
40300 \\
40300 \\
41500 \\
41200\end{array}$ & $\begin{array}{l}38900 \\
42400 \\
43600 \\
44000 \\
45300\end{array}$ & $\begin{array}{l}28000 \\
31400 \\
36100 \\
37200 \\
35600\end{array}$ & $\begin{array}{l}33700 \\
35600 \\
39300 \\
40400 \\
41600\end{array}$ \\
\hline $\begin{array}{r}6 \\
7 \\
8 \\
9 \\
10\end{array}$ & $\begin{array}{l}43400 \\
42500 \\
42300 \\
43100 \\
43000\end{array}$ & $\begin{array}{l}41600 \\
40600 \\
40200 \\
41000 \\
41600\end{array}$ & $\begin{array}{l}42700 \\
41600 \\
41400 \\
42100 \\
42400\end{array}$ & $\begin{array}{l}44700 \\
43400 \\
42300 \\
41900 \\
40500\end{array}$ & $\begin{array}{l}40600 \\
40500 \\
40900 \\
39800 \\
38700\end{array}$ & $\begin{array}{l}42400 \\
42200 \\
41600 \\
40500 \\
39800\end{array}$ & $\begin{array}{r}44000 \\
--- \\
--- \\
--- \\
---\end{array}$ & $\begin{array}{r}39800 \\
--- \\
--- \\
--- \\
---\end{array}$ & $\begin{array}{c}42100 \\
--- \\
--- \\
--- \\
---\end{array}$ \\
\hline $\begin{array}{l}11 \\
12 \\
13 \\
14 \\
15\end{array}$ & $\begin{array}{l}43600 \\
46500 \\
47400 \\
47000 \\
47000\end{array}$ & $\begin{array}{l}41500 \\
42000 \\
42100 \\
46100 \\
46000\end{array}$ & $\begin{array}{l}42400 \\
43200 \\
44500 \\
46600 \\
46500\end{array}$ & $\begin{array}{l}41100 \\
44500 \\
44800 \\
44600 \\
44200\end{array}$ & $\begin{array}{l}38100 \\
38900 \\
40300 \\
42900 \\
42800\end{array}$ & $\begin{array}{l}39400 \\
40700 \\
42300 \\
43900 \\
43700\end{array}$ & $\begin{array}{l}--- \\
--- \\
--- \\
--- \\
---\end{array}$ & $\begin{array}{l}--- \\
--- \\
--- \\
--- \\
---\end{array}$ & $\begin{array}{l}--- \\
--- \\
--- \\
--- \\
---\end{array}$ \\
\hline $\begin{array}{l}16 \\
17 \\
18 \\
19 \\
20\end{array}$ & $\begin{array}{l}47000 \\
46700 \\
46700 \\
46900 \\
46700\end{array}$ & $\begin{array}{l}45800 \\
45200 \\
45600 \\
45800 \\
45400\end{array}$ & $\begin{array}{l}46400 \\
46200 \\
46200 \\
46300 \\
46000\end{array}$ & $\begin{array}{l}44500 \\
43700 \\
43800 \\
43400 \\
42900\end{array}$ & $\begin{array}{l}42000 \\
41700 \\
41000 \\
38400 \\
37300\end{array}$ & $\begin{array}{l}43600 \\
42800 \\
42400 \\
40900 \\
39800\end{array}$ & $\begin{array}{l}--- \\
--- \\
--- \\
--- \\
---\end{array}$ & $\begin{array}{l}--- \\
--- \\
--- \\
--- \\
---\end{array}$ & $\begin{array}{l}--- \\
--- \\
--- \\
--- \\
---\end{array}$ \\
\hline $\begin{array}{l}21 \\
22 \\
23 \\
24 \\
25\end{array}$ & $\begin{array}{l}46000 \\
43500 \\
43100 \\
40300 \\
38900\end{array}$ & $\begin{array}{l}42600 \\
40000 \\
38900 \\
38600 \\
34900\end{array}$ & $\begin{array}{l}44900 \\
41800 \\
39900 \\
39300 \\
37800\end{array}$ & $\begin{array}{l}41000 \\
39400 \\
36100 \\
36000 \\
37600\end{array}$ & $\begin{array}{l}36200 \\
34700 \\
33600 \\
33500 \\
33200\end{array}$ & $\begin{array}{l}38100 \\
37100 \\
35100 \\
34800 \\
34000\end{array}$ & $\begin{array}{l}--- \\
--- \\
--- \\
--- \\
---\end{array}$ & $\begin{array}{l}--- \\
--- \\
--- \\
--- \\
---\end{array}$ & $\begin{array}{l}--- \\
--- \\
--- \\
--- \\
---\end{array}$ \\
\hline $\begin{array}{l}26 \\
27 \\
28 \\
29 \\
30 \\
31\end{array}$ & $\begin{array}{r}36200 \\
35000 \\
34300 \\
34300 \\
34200 \\
----\end{array}$ & $\begin{array}{r}34300 \\
33200 \\
33000 \\
33000 \\
33400 \\
----\end{array}$ & $\begin{array}{r}35300 \\
34100 \\
33500 \\
33700 \\
33900 \\
----\end{array}$ & $\begin{array}{l}34600 \\
36700 \\
37000 \\
36200 \\
37900 \\
37200\end{array}$ & $\begin{array}{l}33000 \\
33100 \\
34800 \\
34600 \\
35700 \\
23500\end{array}$ & $\begin{array}{l}34200 \\
35000 \\
35600 \\
35500 \\
36800 \\
36200\end{array}$ & $\begin{array}{l}--- \\
--- \\
--- \\
--- \\
--- \\
---\end{array}$ & $\begin{array}{l}--- \\
--- \\
--- \\
--- \\
--- \\
---\end{array}$ & $\begin{array}{l}--- \\
--- \\
--- \\
--- \\
--- \\
---\end{array}$ \\
\hline
\end{tabular}


Table A20. Specific conductance and water temperature data collected May-November 2001 at station 101 (USGS identifier 01022820) Northeast Creek at Route 3 bridge near Bar Harbor, ME - Continued.

DEEP

Specific conductance, water, microsiemens per centimeter at 25 degrees Celsius

\begin{tabular}{|c|c|c|c|c|c|c|c|c|c|c|c|c|}
\hline \multirow[t]{2}{*}{ DAY } & MAX & MIN & MEAN & MAX & MIN & MEAN & MAX & MIN & MEAN & MAX & MIN & MEAN \\
\hline & & MAY & & & \multicolumn{3}{|l|}{ JUNE } & \multicolumn{3}{|l|}{ JULY } & \multicolumn{2}{|l|}{ AUGUST } \\
\hline 1 & --- & --- & --- & 40200 & 21100 & 26500 & 45100 & 38800 & 42100 & 46900 & 43800 & 45200 \\
\hline 2 & --- & --- & --- & 44300 & 23800 & 35500 & 46300 & 39800 & 44500 & 46900 & 44700 & 46100 \\
\hline 3 & --- & --- & --- & 44100 & 14500 & 35600 & 45700 & 39100 & 44100 & 47600 & 45000 & 46400 \\
\hline 4 & --- & --- & --- & 42200 & 11000 & 31500 & 45100 & 39200 & 43800 & 47300 & 45500 & 46600 \\
\hline 5 & --- & --- & --- & 37000 & 3240 & 26900 & 46700 & 41700 & 45700 & 47700 & 45000 & 46600 \\
\hline 6 & --- & --- & --- & 30900 & 3300 & 20800 & 47200 & 41900 & 46400 & 47700 & 45800 & 46700 \\
\hline 7 & --- & --- & --- & 28600 & 11000 & 21900 & 45700 & 41500 & 45000 & 47600 & 45700 & 46500 \\
\hline 8 & --- & --- & --- & 33600 & 13700 & 29500 & 45700 & 42400 & 45100 & 47900 & 45800 & 46700 \\
\hline 9 & --- & --- & --- & 36800 & 23400 & 33700 & 44900 & 41700 & 44100 & 46900 & 44600 & 46200 \\
\hline 10 & --- & --- & --- & 35400 & 28400 & 33200 & 43600 & 40300 & 41300 & 46100 & 43800 & 45000 \\
\hline 11 & --- & --- & --- & 31900 & 26500 & 29100 & 43500 & 41500 & 42600 & 46200 & 40000 & 44700 \\
\hline 12 & --- & --- & --- & 27400 & 22900 & 25000 & 43400 & 41700 & 42700 & 42700 & 39400 & 41200 \\
\hline 13 & --- & --- & --- & 23600 & 18700 & 21300 & 43200 & 41100 & 42100 & 44400 & 40300 & 41700 \\
\hline 14 & --- & --- & --- & 19400 & 15700 & 17700 & 43100 & 38800 & 41800 & 47700 & 41600 & 43500 \\
\hline 15 & --- & --- & --- & 17000 & 14300 & 15500 & 38800 & 36500 & 37500 & 49600 & 42800 & 46100 \\
\hline 16 & --- & --- & --- & 15000 & 8380 & 11400 & 41900 & 35000 & 37100 & 49900 & 47800 & 48900 \\
\hline 17 & --- & --- & --- & 26900 & 7230 & 11500 & 46700 & 38500 & 41200 & 49600 & 47000 & 48400 \\
\hline 18 & --- & --- & --- & 24900 & 10800 & 18700 & 46700 & 39700 & 44800 & 48900 & 47400 & 48000 \\
\hline 19 & --- & --- & --- & 34200 & 2620 & 11300 & 47400 & 41900 & 46100 & 49200 & 47500 & 48500 \\
\hline 20 & --- & --- & --- & 39100 & 23800 & 30900 & 47800 & 43900 & 45800 & 49800 & 46200 & 49000 \\
\hline 21 & --- & --- & --- & 42200 & 28200 & 36300 & 47800 & 45400 & 46800 & 49600 & 48400 & 49000 \\
\hline 22 & --- & --- & --- & 44700 & 29800 & 40400 & 48300 & 46500 & 47500 & 50000 & 48700 & 49500 \\
\hline 23 & --- & --- & --- & 46300 & 27000 & 41600 & 49000 & 46000 & 47800 & 50100 & 48000 & 49100 \\
\hline 24 & --- & --- & -- & 46300 & 27000 & 42500 & 49000 & 46600 & 47800 & 50000 & 48400 & 49300 \\
\hline 25 & --- & --- & -- & 44500 & 29000 & 39900 & 48700 & 44200 & 47000 & 49600 & 46400 & 48100 \\
\hline 26 & --- & --- & --- & 41800 & 29900 & 37700 & 46300 & 43300 & 44800 & 49000 & 45700 & 47500 \\
\hline 27 & --- & --- & --- & 40300 & 32200 & 36900 & 44200 & 42100 & 43200 & 48700 & 44800 & 46500 \\
\hline 28 & --- & --- & --- & 39800 & 31200 & 35600 & 44200 & 40400 & 42000 & 48700 & 46700 & 47700 \\
\hline 29 & --- & --- & --- & 41200 & 33300 & 37000 & 43000 & 39900 & 41200 & 48700 & 46500 & 47700 \\
\hline 30 & --- & --- & --- & 42900 & 34600 & 39500 & 44300 & 39100 & 41600 & 47900 & 45600 & 46 \\
\hline 31 & --- & --- & --- & --- & --- & --- & 45600 & 40300 & 43400 & 48900 & 44700 & 47100 \\
\hline MONTH & --- & --- & --- & 46300 & 2620 & 29200 & 49000 & 35000 & 43800 & 50100 & 39400 & 46800 \\
\hline
\end{tabular}

\begin{tabular}{|c|c|c|c|c|c|c|c|c|c|}
\hline \multirow[t]{2}{*}{ DAY } & MAX & MIN & MEAN & MAX & MIN & MEAN & MAX & MIN & MEAN \\
\hline & \multicolumn{3}{|c|}{ SEPTEMBER } & \multicolumn{3}{|c|}{ OCTOBER } & \multicolumn{3}{|c|}{ NOVEMBER } \\
\hline 1 & 49100 & 46400 & 48200 & 46200 & 39100 & 41800 & 42300 & 38100 & 40700 \\
\hline 2 & 47200 & 45500 & 46400 & 46100 & 43700 & 45000 & 45100 & 40500 & 43400 \\
\hline 3 & 47600 & 44900 & 46300 & 45700 & 43900 & 44800 & 45400 & 42200 & 44800 \\
\hline 4 & 47800 & 44500 & 46400 & 46700 & 43700 & 45800 & 45900 & 34700 & 45100 \\
\hline 5 & 47800 & 46100 & 46900 & 47100 & 44100 & 46000 & 46900 & 42200 & 45800 \\
\hline 6 & 47000 & 45200 & 46000 & 47800 & 43300 & 46000 & 46600 & 44000 & 45400 \\
\hline 7 & 46600 & 44700 & 45600 & 47400 & 44800 & 46000 & -- & -- & -- \\
\hline 8 & 46500 & 44100 & 45300 & 46100 & 44000 & 44800 & --- & --- & --- \\
\hline 9 & 45400 & 43600 & 44300 & 45000 & 42700 & 43800 & --- & --- & --- \\
\hline 10 & 44900 & 43100 & 44100 & 43600 & 41400 & 42900 & --- & --- & --- \\
\hline 11 & 45200 & 42600 & 43800 & 44700 & 41100 & 42700 & --- & --- & --- \\
\hline 12 & 47900 & 42700 & 45000 & 46200 & 43400 & 44800 & --- & --- & --- \\
\hline 13 & 48200 & 43000 & 45200 & 47100 & 42600 & 45100 & --- & --- & --- \\
\hline 14 & 48200 & 47200 & 47700 & 47200 & 45600 & 46500 & --- & --- & --- \\
\hline 15 & 48100 & 47000 & 47600 & 47200 & 45400 & 46400 & --- & --- & --- \\
\hline 16 & 47900 & 46800 & 47400 & 47400 & 45500 & 46600 & --- & --- & --- \\
\hline 17 & 47900 & 46700 & 47400 & 46800 & 44500 & 45800 & --- & --- & --- \\
\hline 18 & 48000 & 46500 & 47300 & 47100 & 44300 & 45600 & --- & --- & --- \\
\hline 19 & 47900 & 46500 & 47200 & 46800 & 42200 & 44700 & --- & --- & --- \\
\hline 20 & 47800 & 46400 & 47000 & 46900 & 42200 & 45400 & --- & --- & --- \\
\hline 21 & 47400 & 44600 & 46300 & 46700 & 42500 & 45400 & --- & --- & --- \\
\hline 22 & 45200 & 42000 & 44200 & 45400 & 43800 & 44500 & --- & --- & --- \\
\hline 23 & 44000 & 42100 & 43200 & 45200 & 38200 & 40700 & --- & --- & --- \\
\hline 24 & 43700 & 41600 & 42600 & 39500 & 38700 & 39100 & --- & --- & --- \\
\hline 25 & 42600 & 38000 & 39500 & 42900 & 36800 & 39900 & --- & -- & --- \\
\hline 26 & 40200 & 38100 & 39300 & 42500 & 39900 & 41600 & --- & --- & --- \\
\hline 27 & 40300 & 39100 & 39500 & 42600 & 41000 & 41500 & --- & --- & --- \\
\hline 28 & 40600 & 38700 & 39400 & 42100 & 40000 & 40900 & --- & -- & --- \\
\hline 29 & 40300 & 37900 & 39200 & 40900 & 39000 & 39800 & --- & --- & --- \\
\hline 30 & 40000 & 34800 & 37500 & 41100 & 38600 & 39900 & --- & --- & --- \\
\hline 31 & 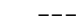 & --- & --- & 41800 & 36500 & 39100 & --- & --- & -- \\
\hline MON' & 49100 & 34800 & 44500 & 47800 & 36500 & 43600 & & & \\
\hline
\end{tabular}


Table A20. Specific conductance and water temperature data collected May-November 2001 at station 101 (USGS identifier 01022820) Northeast Creek at Route 3 bridge near Bar Harbor, ME - Continued.

\section{SHALLOW}

Temperature, water, degrees Celsius

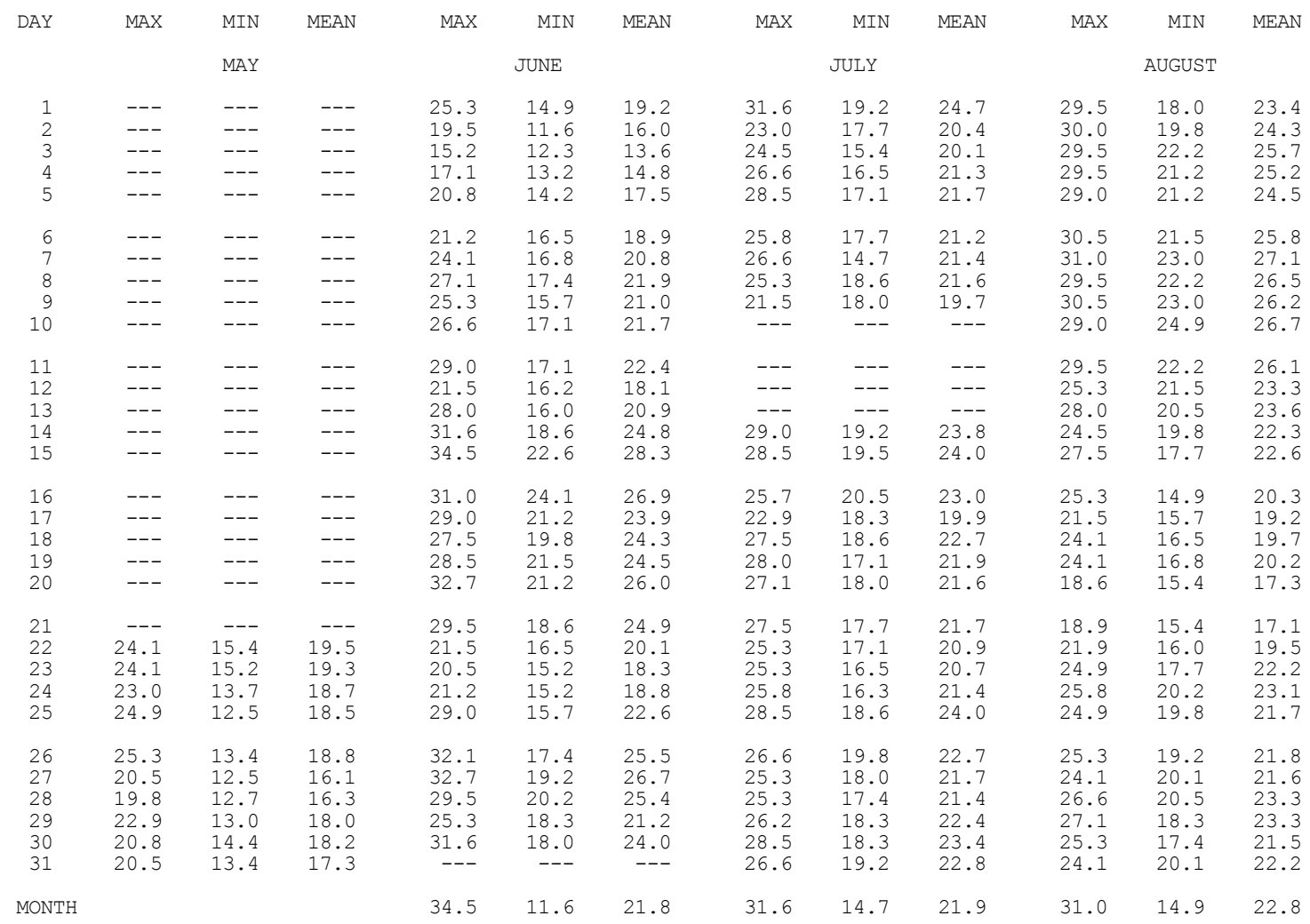

\begin{tabular}{|c|c|c|c|c|c|c|c|c|c|}
\hline DAY & MAX & MIN & MEAN & MAX & MIN & MEAN & MAX & MIN & MEAN \\
\hline & \multicolumn{3}{|c|}{ SEPTEMBER } & \multicolumn{3}{|c|}{ OCTOBER } & \multicolumn{3}{|c|}{ NOVEMBER } \\
\hline $\begin{array}{l}1 \\
2 \\
3 \\
4 \\
5\end{array}$ & $\begin{array}{l}23.3 \\
23.0 \\
22.6 \\
22.2 \\
22.6\end{array}$ & $\begin{array}{l}16.2 \\
13.2 \\
13.7 \\
17.1 \\
15.7\end{array}$ & $\begin{array}{l}20.7 \\
18.7 \\
18.8 \\
19.8 \\
19.5\end{array}$ & $\begin{array}{l}17.4 \\
17.4 \\
18.6 \\
19.8 \\
19.8\end{array}$ & $\begin{array}{r}9.7 \\
9.1 \\
10.5 \\
14.7 \\
14.9\end{array}$ & $\begin{array}{l}13.7 \\
13.6 \\
15.2 \\
17.3 \\
17.4\end{array}$ & $\begin{array}{r}9.1 \\
13.7 \\
13.4 \\
12.7 \\
10.1\end{array}$ & $\begin{array}{r}6.0 \\
9.1 \\
10.3 \\
9.7 \\
8.5\end{array}$ & $\begin{array}{r}7.8 \\
11.8 \\
12.2 \\
10.9 \\
9.3\end{array}$ \\
\hline $\begin{array}{r}6 \\
7 \\
8 \\
9 \\
10\end{array}$ & $\begin{array}{l}21.9 \\
24.1 \\
26.6 \\
28.0 \\
26.6\end{array}$ & $\begin{array}{l}14.9 \\
16.5 \\
18.6 \\
21.5 \\
22.2\end{array}$ & $\begin{array}{l}18.6 \\
20.6 \\
22.9 \\
24.4 \\
23.9\end{array}$ & $\begin{array}{l}17.4 \\
16.0 \\
14.4 \\
12.7 \\
13.7\end{array}$ & $\begin{array}{r}11.8 \\
10.3 \\
8.9 \\
6.8 \\
9.7\end{array}$ & $\begin{array}{l}15.5 \\
13.2 \\
12.1 \\
10.4 \\
11.9\end{array}$ & $\begin{array}{l}9.9 \\
--- \\
--- \\
--- \\
---\end{array}$ & $\begin{array}{l}6.7 \\
--- \\
--- \\
--- \\
---\end{array}$ & $\begin{array}{l}8.4 \\
--- \\
--- \\
--- \\
---\end{array}$ \\
\hline $\begin{array}{l}11 \\
12 \\
13 \\
14 \\
15\end{array}$ & $\begin{array}{l}25.8 \\
24.1 \\
22.6 \\
18.3 \\
17.1\end{array}$ & $\begin{array}{l}19.5 \\
16.8 \\
16.2 \\
13.7 \\
12.9\end{array}$ & $\begin{array}{l}23.2 \\
20.6 \\
19.5 \\
16.7 \\
15.0\end{array}$ & $\begin{array}{l}16.5 \\
18.6 \\
16.3 \\
13.4 \\
14.2\end{array}$ & $\begin{array}{r}9.9 \\
11.8 \\
12.7 \\
11.2 \\
12.3\end{array}$ & $\begin{array}{l}13.4 \\
15.2 \\
14.2 \\
12.7 \\
13.4\end{array}$ & $\begin{array}{l}--- \\
--- \\
--- \\
--- \\
---\end{array}$ & $\begin{array}{l}--- \\
--- \\
--- \\
--- \\
---\end{array}$ & $\begin{array}{l}--- \\
--- \\
--- \\
--- \\
---\end{array}$ \\
\hline $\begin{array}{l}16 \\
17 \\
18 \\
19 \\
20\end{array}$ & $\begin{array}{l}18.0 \\
17.7 \\
18.0 \\
16.5 \\
16.5\end{array}$ & $\begin{array}{l}13.7 \\
13.2 \\
14.2 \\
14.2 \\
14.2\end{array}$ & $\begin{array}{l}15.4 \\
15.4 \\
16.0 \\
15.6 \\
15.4\end{array}$ & $\begin{array}{l}14.2 \\
14.4 \\
11.4 \\
10.9 \\
12.3\end{array}$ & $\begin{array}{r}11.4 \\
11.2 \\
9.1 \\
7.4 \\
8.9\end{array}$ & $\begin{array}{r}12.7 \\
13.2 \\
10.7 \\
9.4 \\
10.6\end{array}$ & $\begin{array}{l}--- \\
--- \\
--- \\
--- \\
---\end{array}$ & $\begin{array}{l}--- \\
--- \\
--- \\
--- \\
---\end{array}$ & $\begin{array}{l}--- \\
--- \\
--- \\
--- \\
---\end{array}$ \\
\hline $\begin{array}{l}21 \\
22 \\
23 \\
24 \\
25\end{array}$ & $\begin{array}{l}17.7 \\
21.2 \\
24.1 \\
23.3 \\
19.5\end{array}$ & $\begin{array}{l}14.9 \\
17.1 \\
18.9 \\
18.3 \\
18.6\end{array}$ & $\begin{array}{l}16.8 \\
19.0 \\
21.0 \\
20.6 \\
18.8\end{array}$ & $\begin{array}{l}13.7 \\
14.7 \\
11.8 \\
14.4 \\
14.9\end{array}$ & $\begin{array}{r}7.8 \\
8.3 \\
7.0 \\
11.6 \\
12.3\end{array}$ & $\begin{array}{l}11.4 \\
12.1 \\
10.3 \\
13.3 \\
13.8\end{array}$ & $\begin{array}{l}--- \\
--- \\
--- \\
--- \\
---\end{array}$ & $\begin{array}{l}--- \\
--- \\
--- \\
--- \\
---\end{array}$ & $\begin{array}{l}--- \\
--- \\
--- \\
--- \\
---\end{array}$ \\
\hline $\begin{array}{l}26 \\
27 \\
28 \\
29 \\
30 \\
31\end{array}$ & $\begin{array}{r}22.2 \\
18.6 \\
17.4 \\
17.4 \\
17.7 \\
---\end{array}$ & $\begin{array}{r}17.4 \\
16.0 \\
13.4 \\
12.2 \\
11.2 \\
---\end{array}$ & $\begin{array}{r}19.7 \\
17.1 \\
15.8 \\
14.7 \\
14.1 \\
---\end{array}$ & $\begin{array}{r}15.2 \\
13.0 \\
11.6 \\
8.9 \\
10.5 \\
8.1\end{array}$ & $\begin{array}{r}10.7 \\
8.1 \\
6.2 \\
5.2 \\
4.3 \\
3.7\end{array}$ & $\begin{array}{r}12.6 \\
10.7 \\
8.6 \\
7.6 \\
7.7 \\
6.3\end{array}$ & $\begin{array}{l}--- \\
--- \\
--- \\
--- \\
--- \\
---\end{array}$ & $\begin{array}{l}--- \\
--- \\
--- \\
--- \\
--- \\
---\end{array}$ & $\begin{array}{l}--- \\
--- \\
--- \\
--- \\
--- \\
---\end{array}$ \\
\hline $\mathrm{ON}$ & & 2 & & & & & & & \\
\hline
\end{tabular}


Table A20. Specific conductance and water temperature data collected May-November 2001 at station 101 (USGS identifier 01022820) Northeast Creek at Route 3 bridge near Bar Harbor, ME - Continued.

\begin{tabular}{|c|c|c|c|c|c|c|c|c|c|c|c|c|}
\hline \multirow[b]{2}{*}{ DAY } & \multicolumn{12}{|c|}{ DEEP } \\
\hline & MAX & MIN & MEAN & MAX & MIN & MEAN & MAX & MIN & MEAN & MAX & MIN & MEAN \\
\hline & & MAY & & & JUNE & & & JULY & & & AUGUST & \\
\hline 1 & --- & --- & --- & 20.8 & 15.4 & 18.1 & 26.2 & 19.2 & 23.0 & 28.0 & 21.5 & 24.1 \\
\hline 2 & --- & --- & --- & 19.8 & 11.6 & 16.9 & 23.7 & 18.6 & 20.7 & 27.1 & 21.9 & 24.1 \\
\hline 3 & --- & --- & --- & 14.4 & 11.8 & 12.7 & 22.2 & 16.5 & 19.8 & 27.1 & 21.9 & 24.1 \\
\hline 4 & --- & --- & --- & 16.0 & 13.0 & 14.1 & 22.6 & 16.5 & 19.0 & 26.6 & 20.8 & 23.4 \\
\hline 5 & --- & --- & --- & 19.5 & 14.2 & 16.3 & 24.1 & 16.8 & 19.1 & 25.8 & 20.5 & 22.9 \\
\hline 6 & --- & --- & --- & 20.2 & 16.3 & 18.1 & 23.0 & 17.4 & 19.5 & 27.5 & 21.9 & 24.6 \\
\hline 7 & --- & --- & --- & 21.9 & 16.8 & 18.9 & 23.0 & 17.4 & 19.9 & 28.0 & 21.9 & 25.2 \\
\hline 8 & --- & --- & --- & 20.8 & 17.4 & 19.1 & 23.3 & 18.0 & 19.9 & 29.5 & 23.7 & 26.8 \\
\hline 9 & --- & --- & --- & 20.8 & 16.0 & 18.1 & 21.9 & 16.8 & 18.5 & 28.5 & 23.0 & 26.0 \\
\hline 10 & --- & --- & --- & 20.2 & 16.2 & 18.4 & 20.2 & 17.7 & 19.3 & 29.5 & 26.2 & 27.8 \\
\hline 11 & --- & --- & --- & 22.2 & 19.2 & 20.5 & 22.2 & 18.9 & 20.9 & 31.6 & 25.7 & 28.5 \\
\hline 12 & --- & --- & --- & 21.5 & 18.6 & 19.9 & 25.7 & 21.5 & 23.5 & 25.8 & 22.2 & 24.1 \\
\hline 13 & --- & --- & --- & 21.2 & 18.3 & 19.2 & 28.0 & 24.1 & 26.1 & 27.1 & 22.2 & 24.1 \\
\hline 14 & --- & --- & --- & 24.1 & 19.8 & 21.4 & 29.5 & 26.6 & 27.9 & 25.8 & 21.9 & 23.6 \\
\hline 15 & --- & --- & --- & 26.6 & 23.0 & 24.3 & 29.5 & 27.0 & 28.2 & 26.2 & 19.5 & 22.5 \\
\hline 16 & --- & --- & --- & 29.5 & 24.5 & 26.7 & 28.0 & 23.0 & 26.8 & 26.2 & 19.2 & 23.1 \\
\hline 17 & --- & --- & --- & 27.1 & 22.6 & 24.7 & 24.5 & 18.0 & 23.2 & 21.5 & 15.7 & 19.1 \\
\hline 18 & --- & --- & --- & 25.8 & 22.2 & 23.7 & 23.7 & 18.3 & 20.5 & 23.4 & 16.5 & 19.1 \\
\hline 19 & --- & --- & --- & 27.5 & 21.5 & 24.1 & 25.3 & 18.9 & 21.3 & 24.1 & 16.8 & 19.9 \\
\hline 20 & --- & --- & --- & 24.9 & 20.5 & 22.9 & 24.9 & 18.6 & 20.1 & 18.6 & 15.4 & 17.3 \\
\hline 21 & --- & --- & --- & 24.5 & 18.6 & 22.0 & 27.1 & 17.7 & 21.1 & 18.9 & 15.4 & 17.0 \\
\hline 22 & 20.5 & 15.4 & 18.4 & 20.1 & 16.5 & 18.7 & 25.3 & 16.8 & 20.5 & 21.5 & 16.0 & 19.4 \\
\hline 23 & 19.8 & 15.2 & 17.0 & 19.5 & 15.2 & 16.6 & 25.3 & 16.3 & 20.5 & 24.1 & 17.7 & 21.8 \\
\hline 24 & 19.5 & 13.9 & 16.6 & 19.5 & 15.2 & 16.7 & 24.9 & 16.3 & 21.2 & 25.8 & 20.2 & 22.9 \\
\hline 25 & 17.7 & 12.7 & 15.0 & 23.3 & 15.5 & 19.0 & 28.0 & 18.3 & 23.5 & 24.9 & 19.8 & 21.9 \\
\hline 26 & 19.5 & 13.7 & 16.2 & 25.8 & 17.4 & 21.5 & 25.8 & 19.5 & 22.2 & 24.9 & 19.8 & 22.2 \\
\hline 27 & 20.5 & 12.5 & 15.1 & 27.5 & 18.6 & 23.1 & 25.3 & 19.5 & 22.1 & 23.0 & 20.1 & 21.3 \\
\hline 28 & 17.1 & 12.7 & 14.8 & 28.0 & 21.2 & 23.8 & 25.8 & 19.8 & 22.8 & 25.8 & 21.5 & 23.3 \\
\hline 29 & 20.5 & 13.0 & 16.3 & 23.7 & 19.2 & 21.9 & 27.1 & 21.5 & 24.0 & 26.6 & 21.8 & 24.3 \\
\hline 30 & 19.8 & 14.2 & 17.3 & 24.5 & 19.5 & 21.1 & 29.0 & 22.2 & 25.0 & 26.2 & 19.8 & 22.7 \\
\hline 31 & 18.9 & 13.4 & 17.2 & --- & --- & --- & 26.6 & 21.5 & 24.2 & 24.1 & 19.5 & 22.0 \\
\hline MONTH & & & & 29.5 & 11.6 & 20.1 & 29.5 & 16.3 & 22.1 & 31.6 & 15.4 & 22.9 \\
\hline
\end{tabular}

\begin{tabular}{|c|c|c|c|c|c|c|c|c|c|}
\hline DAY & MAX & MIN & MEAN & MAX & MIN & MEAN & MAX & MIN & MEAN \\
\hline & \multicolumn{3}{|c|}{ SEPTEMBER } & \multicolumn{3}{|c|}{ OCTOBER } & \multicolumn{3}{|c|}{ NOVEMBER } \\
\hline 1 & 22.2 & 17.7 & 20.5 & 16.8 & 13.2 & 15.0 & 10.1 & 7.4 & 8.5 \\
\hline 2 & 22.6 & 17.4 & 19.7 & 16.5 & 13.4 & 14.9 & 12.0 & 8.7 & 10.9 \\
\hline 3 & 22.2 & 16.8 & 19.7 & 17.7 & 14.9 & 16.0 & 12.5 & 11.8 & 12.3 \\
\hline 4 & 21.2 & 17.1 & 19.3 & 18.3 & 15.2 & 17.1 & 13.2 & 10.7 & 11.7 \\
\hline 5 & 21.9 & 18.0 & 19.7 & 18.3 & 16.8 & 17.6 & 11.6 & 9.3 & 10.3 \\
\hline 6 & 21.2 & 16.5 & 19.0 & 17.4 & 14.2 & 15.9 & 10.5 & 7.8 & 9.1 \\
\hline 7 & 23.0 & 17.1 & 20.7 & 16.2 & 12.2 & 14.3 & --- & --- & --- \\
\hline 8 & 25.3 & 21.2 & 23.2 & 14.7 & 10.1 & 12.8 & --- & --- & --- \\
\hline 9 & 26.6 & 21.5 & 24.1 & 13.0 & 9.3 & 11.1 & --- & --- & --- \\
\hline 10 & 25.8 & 22.6 & 23.9 & 13.0 & 10.7 & 11.9 & --- & --- & --- \\
\hline 11 & 25.8 & 20.5 & 23.7 & 16.0 & 10.5 & 13.4 & --- & --- & --- \\
\hline 12 & 23.7 & 18.0 & 21.7 & 17.7 & 14.2 & 16.3 & --- & --- & --- \\
\hline 13 & 22.2 & 16.8 & 19.5 & 16.8 & 12.5 & 14.9 & --- & --- & --- \\
\hline 14 & 18.6 & 13.9 & 16.8 & 13.4 & 11.6 & 12.7 & --- & --- & --- \\
\hline 15 & 17.1 & 12.9 & 14.9 & 14.2 & 12.0 & 13.3 & --- & --- & --- \\
\hline 16 & 17.7 & 13.4 & 15.1 & 13.9 & 11.4 & 12.6 & --- & --- & --- \\
\hline 17 & 17.7 & 13.2 & 15.3 & 14.4 & 11.2 & 13.1 & --- & --- & --- \\
\hline 18 & 17.7 & 14.2 & 15.9 & 11.6 & 9.1 & 10.6 & --- & --- & --- \\
\hline 19 & 16.5 & 13.9 & 15.5 & 10.9 & 7.4 & 9.4 & --- & --- & --- \\
\hline 20 & 16.3 & 13.9 & 15.3 & 12.0 & 9.7 & 11.2 & --- & --- & --- \\
\hline 21 & 17.7 & 14.9 & 16.6 & 13.4 & 11.6 & 12.9 & --- & --- & --- \\
\hline 22 & 20.5 & 17.1 & 18.8 & 15.7 & 13.2 & 14.1 & --- & --- & --- \\
\hline 23 & 23.3 & 19.5 & 21.3 & 13.7 & 9.7 & 12.0 & --- & --- & --- \\
\hline 24 & 23.0 & 19.8 & 21.5 & 13.2 & 11.6 & 12.4 & --- & --- & --- \\
\hline 25 & 20.5 & 18.6 & 18.9 & 14.7 & 13.2 & 13.9 & --- & --- & -- \\
\hline 26 & 21.5 & 18.6 & 20.0 & 15.7 & 13.7 & 14.4 & --- & --- & --- \\
\hline 27 & 20.1 & 18.0 & 19.2 & 15.2 & 9.1 & 13.8 & --- & --- & --- \\
\hline 28 & 20.1 & 17.7 & 18.9 & 13.7 & 6.7 & 10.8 & --- & --- & --- \\
\hline 29 & 18.9 & 15.7 & 17.5 & 10.3 & 6.2 & 8.4 & --- & --- & - \\
\hline 30 & 17.7 & 12.5 & 15.1 & 11.8 & 7.0 & 9.5 & --- & --- & -1 \\
\hline 31 & --- & --- & --- & 8.3 & 6.0 & 7.4 & --- & -- & \\
\hline MONTH & 26.6 & 12.5 & 19.0 & 18.3 & 6.0 & 13.0 & & & \\
\hline
\end{tabular}


Table A21. Specific conductance and water temperature data collected May-November 2001 at station 102 (USGS identifier 442517068190501) Northeast Creek near Bar Harbor, ME [Data are presented as minimum, mean and maximum daily values of continuous data at 15-minute intervals. Shallow and deep data were collected at fixed depths located, respectively, about $0.2 \mathrm{~m}$ below the water surface and about $0.2 \mathrm{~m}$ above the stream bottom.]

SHALLOW

Specific conductance, water, microsiemens per centimeter at 25 degrees Celsius

\begin{tabular}{|c|c|c|c|c|c|c|c|c|c|c|c|c|}
\hline DAY & MAX & MIN & MEAN & MAX & MIN & MEAN & MAX & MIN & MEAN & MAX & MIN & MEAN \\
\hline $\begin{array}{l}1 \\
2 \\
3 \\
4 \\
5\end{array}$ & $\begin{array}{l}--- \\
--- \\
--- \\
--- \\
---\end{array}$ & $\begin{array}{l}--- \\
--- \\
--- \\
--- \\
---\end{array}$ & $\begin{array}{l}--- \\
--- \\
--- \\
--- \\
---\end{array}$ & $\begin{array}{r}5880 \\
16400 \\
24700 \\
45200 \\
25100\end{array}$ & $\begin{array}{r}2020 \\
2920 \\
1490 \\
937 \\
453\end{array}$ & $\begin{array}{l}2920 \\
4780 \\
7330 \\
5870 \\
1690\end{array}$ & $\begin{array}{l}--- \\
--- \\
--- \\
--- \\
---\end{array}$ & $\begin{array}{l}--- \\
--- \\
--- \\
--- \\
---\end{array}$ & $\begin{array}{l}--- \\
--- \\
--- \\
--- \\
---\end{array}$ & $\begin{array}{l}31200 \\
31600 \\
31400 \\
34200 \\
36100\end{array}$ & $\begin{array}{l}27000 \\
29100 \\
29100 \\
29600 \\
30400\end{array}$ & $\begin{array}{l}28000 \\
30100 \\
29900 \\
31000 \\
32400\end{array}$ \\
\hline $\begin{array}{l}11 \\
12 \\
13 \\
14 \\
15\end{array}$ & $\begin{array}{l}--- \\
--- \\
--- \\
--- \\
---\end{array}$ & $\begin{array}{l}--- \\
--- \\
--- \\
--- \\
---\end{array}$ & $\begin{array}{l}--- \\
--- \\
--- \\
--- \\
---\end{array}$ & $\begin{array}{r}1540 \\
--- \\
47200 \\
7960 \\
1690\end{array}$ & $\begin{array}{l}899 \\
--- \\
870 \\
909 \\
923\end{array}$ & $\begin{array}{r}970 \\
--- \\
3380 \\
1320 \\
1050\end{array}$ & $\begin{array}{c}--- \\
--- \\
--- \\
8180 \\
---\end{array}$ & $\begin{array}{r}--- \\
--- \\
--- \\
3720 \\
---\end{array}$ & $\begin{array}{r}--- \\
--- \\
--- \\
4320 \\
---\end{array}$ & $\begin{array}{l}30700 \\
30200 \\
30300 \\
30700 \\
37100\end{array}$ & $\begin{array}{l}29000 \\
29400 \\
29300 \\
29200 \\
30200\end{array}$ & $\begin{array}{l}29800 \\
29800 \\
29800 \\
29800 \\
31700\end{array}$ \\
\hline $\begin{array}{l}21 \\
22 \\
23 \\
24 \\
25\end{array}$ & $\begin{array}{r}--- \\
12200 \\
6370 \\
10000 \\
10000\end{array}$ & $\begin{array}{r}--- \\
599 \\
1290 \\
2700 \\
3030\end{array}$ & $\begin{array}{r}--- \\
1040 \\
2340 \\
7160 \\
7030\end{array}$ & $\begin{array}{r}31900 \\
--- \\
--- \\
--- \\
---\end{array}$ & $\begin{array}{l}729 \\
---- \\
--- \\
--- \\
---\end{array}$ & $\begin{array}{c}3240 \\
--- \\
--- \\
--- \\
---\end{array}$ & $\begin{array}{l}--- \\
--- \\
--- \\
--- \\
---\end{array}$ & $\begin{array}{l}--- \\
--- \\
--- \\
--- \\
---\end{array}$ & $\begin{array}{l}--- \\
--- \\
--- \\
--- \\
---\end{array}$ & $\begin{array}{l}49100 \\
48800 \\
47800 \\
45500 \\
43600\end{array}$ & $\begin{array}{l}44700 \\
43900 \\
44100 \\
42700 \\
38500\end{array}$ & $\begin{array}{l}47400 \\
46900 \\
45700 \\
44100 \\
41100\end{array}$ \\
\hline $\begin{array}{l}26 \\
27 \\
28 \\
29 \\
30 \\
31\end{array}$ & $\begin{array}{l}10000 \\
10000 \\
10000 \\
10000 \\
10000 \\
10000\end{array}$ & $\begin{array}{l}3170 \\
4960 \\
4820 \\
4360 \\
3880 \\
2520\end{array}$ & $\begin{array}{l}9000 \\
9030 \\
8560 \\
8730 \\
6430 \\
3310\end{array}$ & $\begin{array}{r}--- \\
29700 \\
28800 \\
--- \\
--- \\
---\end{array}$ & $\begin{array}{r}--- \\
6480 \\
7030 \\
--- \\
--- \\
---\end{array}$ & $\begin{array}{r}--- \\
9580 \\
9230 \\
--- \\
--- \\
---\end{array}$ & $\begin{array}{l}43200 \\
37000 \\
30100 \\
27200 \\
27400 \\
28900\end{array}$ & $\begin{array}{l}35100 \\
28100 \\
26600 \\
25000 \\
24200 \\
25700\end{array}$ & $\begin{array}{l}39200 \\
32700 \\
27600 \\
25900 \\
25500 \\
26700\end{array}$ & $\begin{array}{l}40500 \\
39900 \\
40100 \\
40200 \\
40100 \\
39400\end{array}$ & $\begin{array}{r}38900 \\
35000 \\
15000 \\
14000 \\
14100 \\
1120\end{array}$ & $\begin{array}{l}39800 \\
38400 \\
35500 \\
30200 \\
21300 \\
19800\end{array}$ \\
\hline
\end{tabular}

\begin{tabular}{|c|c|c|c|c|c|c|c|c|c|}
\hline \multirow[t]{2}{*}{ DAY } & MAX & MIN & MEAN & MAX & MIN & MEAN & MAX & MIN & MEAN \\
\hline & \multicolumn{3}{|c|}{ SEPTEMBER } & \multicolumn{3}{|c|}{ OCTOBER } & \multicolumn{3}{|c|}{ NOVEMBER } \\
\hline $\begin{array}{l}1 \\
2 \\
3 \\
4 \\
5\end{array}$ & $\begin{array}{l}41300 \\
37900 \\
37900 \\
37400 \\
36800\end{array}$ & $\begin{array}{l}15300 \\
14700 \\
13700 \\
13700 \\
13300\end{array}$ & $\begin{array}{l}25300 \\
24100 \\
25300 \\
24300 \\
20100\end{array}$ & $\begin{array}{l}37200 \\
38100 \\
39000 \\
42200 \\
42400\end{array}$ & $\begin{array}{l}34900 \\
37000 \\
37100 \\
37400 \\
38200\end{array}$ & $\begin{array}{l}35400 \\
37600 \\
37800 \\
38900 \\
39800\end{array}$ & $\begin{array}{l}37300 \\
37400 \\
39600 \\
40200 \\
41600\end{array}$ & $\begin{array}{l}30100 \\
27000 \\
32100 \\
31300 \\
36900\end{array}$ & $\begin{array}{l}35400 \\
32900 \\
35200 \\
36400 \\
39800\end{array}$ \\
\hline $\begin{array}{r}6 \\
7 \\
8 \\
9 \\
10\end{array}$ & $\begin{array}{r}24200 \\
17700 \\
1660 \\
151 \\
17400\end{array}$ & $\begin{array}{l}597 \\
171 \\
112 \\
100 \\
104\end{array}$ & $\begin{array}{r}16600 \\
3190 \\
172 \\
135 \\
533\end{array}$ & $\begin{array}{l}44400 \\
43700 \\
42100 \\
41800 \\
40200\end{array}$ & $\begin{array}{l}38400 \\
39900 \\
39600 \\
40100 \\
39000\end{array}$ & $\begin{array}{l}40900 \\
41300 \\
41000 \\
40700 \\
39700\end{array}$ & $\begin{array}{c}40000 \\
--- \\
--- \\
--- \\
---\end{array}$ & $\begin{array}{c}36800 \\
--- \\
--- \\
--- \\
---\end{array}$ & $\begin{array}{c}38900 \\
--- \\
--- \\
--- \\
--\end{array}$ \\
\hline $\begin{array}{l}11 \\
12 \\
13 \\
14 \\
15\end{array}$ & $\begin{array}{l}38800 \\
38700 \\
42100 \\
47100 \\
47700\end{array}$ & $\begin{array}{r}95 \\
37200 \\
37700 \\
40000 \\
42000\end{array}$ & $\begin{array}{l}22000 \\
38000 \\
39100 \\
41600 \\
44900\end{array}$ & $\begin{array}{l}40800 \\
43700 \\
46600 \\
48800 \\
48500\end{array}$ & $\begin{array}{l}38800 \\
38800 \\
41100 \\
44300 \\
44600\end{array}$ & $\begin{array}{l}39600 \\
40800 \\
42900 \\
46700 \\
46900\end{array}$ & $\begin{array}{l}--- \\
--- \\
--- \\
--- \\
---\end{array}$ & $\begin{array}{l}--- \\
--- \\
--- \\
--- \\
---\end{array}$ & $\begin{array}{l}--- \\
--- \\
--- \\
--- \\
---\end{array}$ \\
\hline $\begin{array}{l}16 \\
17 \\
18 \\
19 \\
20\end{array}$ & $\begin{array}{l}48000 \\
48300 \\
48600 \\
48600 \\
48300\end{array}$ & $\begin{array}{l}45400 \\
46200 \\
47000 \\
46700 \\
44900\end{array}$ & $\begin{array}{l}46900 \\
47400 \\
47700 \\
47700 \\
47000\end{array}$ & $\begin{array}{l}48200 \\
48200 \\
46900 \\
44200 \\
43700\end{array}$ & $\begin{array}{l}44200 \\
41800 \\
43100 \\
37400 \\
36300\end{array}$ & $\begin{array}{l}46400 \\
45600 \\
45400 \\
42100 \\
40900\end{array}$ & $\begin{array}{l}--- \\
--- \\
--- \\
--- \\
---\end{array}$ & $\begin{array}{l}--- \\
--- \\
--- \\
--- \\
---\end{array}$ & $\begin{array}{l}--- \\
--- \\
--- \\
--- \\
---\end{array}$ \\
\hline $\begin{array}{l}21 \\
22 \\
23 \\
24 \\
25\end{array}$ & $\begin{array}{l}47500 \\
44100 \\
40800 \\
39200 \\
38500\end{array}$ & $\begin{array}{l}43300 \\
37800 \\
39000 \\
37800 \\
36100\end{array}$ & $\begin{array}{l}45400 \\
41900 \\
39900 \\
38600 \\
37900\end{array}$ & $\begin{array}{l}42100 \\
39100 \\
38800 \\
37000 \\
37200\end{array}$ & $\begin{array}{l}32200 \\
36300 \\
35800 \\
31600 \\
35300\end{array}$ & $\begin{array}{l}38400 \\
38100 \\
37400 \\
35900 \\
36300\end{array}$ & $\begin{array}{l}--- \\
--- \\
--- \\
--- \\
---\end{array}$ & $\begin{array}{l}--- \\
--- \\
--- \\
--- \\
---\end{array}$ & $\begin{array}{l}--- \\
--- \\
--- \\
--- \\
---\end{array}$ \\
\hline $\begin{array}{l}26 \\
27 \\
28 \\
29 \\
30 \\
31\end{array}$ & $\begin{array}{r}36600 \\
34000 \\
33900 \\
35000 \\
35500 \\
----\end{array}$ & $\begin{array}{r}33000 \\
32700 \\
32800 \\
33600 \\
34100 \\
----\end{array}$ & $\begin{array}{r}34300 \\
33300 \\
33300 \\
34200 \\
34800 \\
----\end{array}$ & $\begin{array}{l}36300 \\
36500 \\
37000 \\
37200 \\
37900 \\
38200\end{array}$ & $\begin{array}{l}34300 \\
35000 \\
36100 \\
36200 \\
32600 \\
27500\end{array}$ & $\begin{array}{l}35700 \\
36100 \\
36500 \\
36700 \\
36800 \\
36300\end{array}$ & $\begin{array}{l}--- \\
--- \\
--- \\
--- \\
--- \\
---\end{array}$ & $\begin{array}{l}--- \\
--- \\
--- \\
--- \\
--- \\
---\end{array}$ & $\begin{array}{l}--- \\
--- \\
--- \\
--- \\
--- \\
---\end{array}$ \\
\hline MONTH & 48600 & 95 & 31200 & 48800 & 27500 & 39800 & & & \\
\hline
\end{tabular}


Table A21. Specific conductance and water temperature data collected May-November 2001 at station 102 (USGS identifier 442517068190501) Northeast Creek near Bar Harbor, ME - Continued.

DEEP

Specific conductance, water, microsiemens per centimeter at 25 degrees Celsius

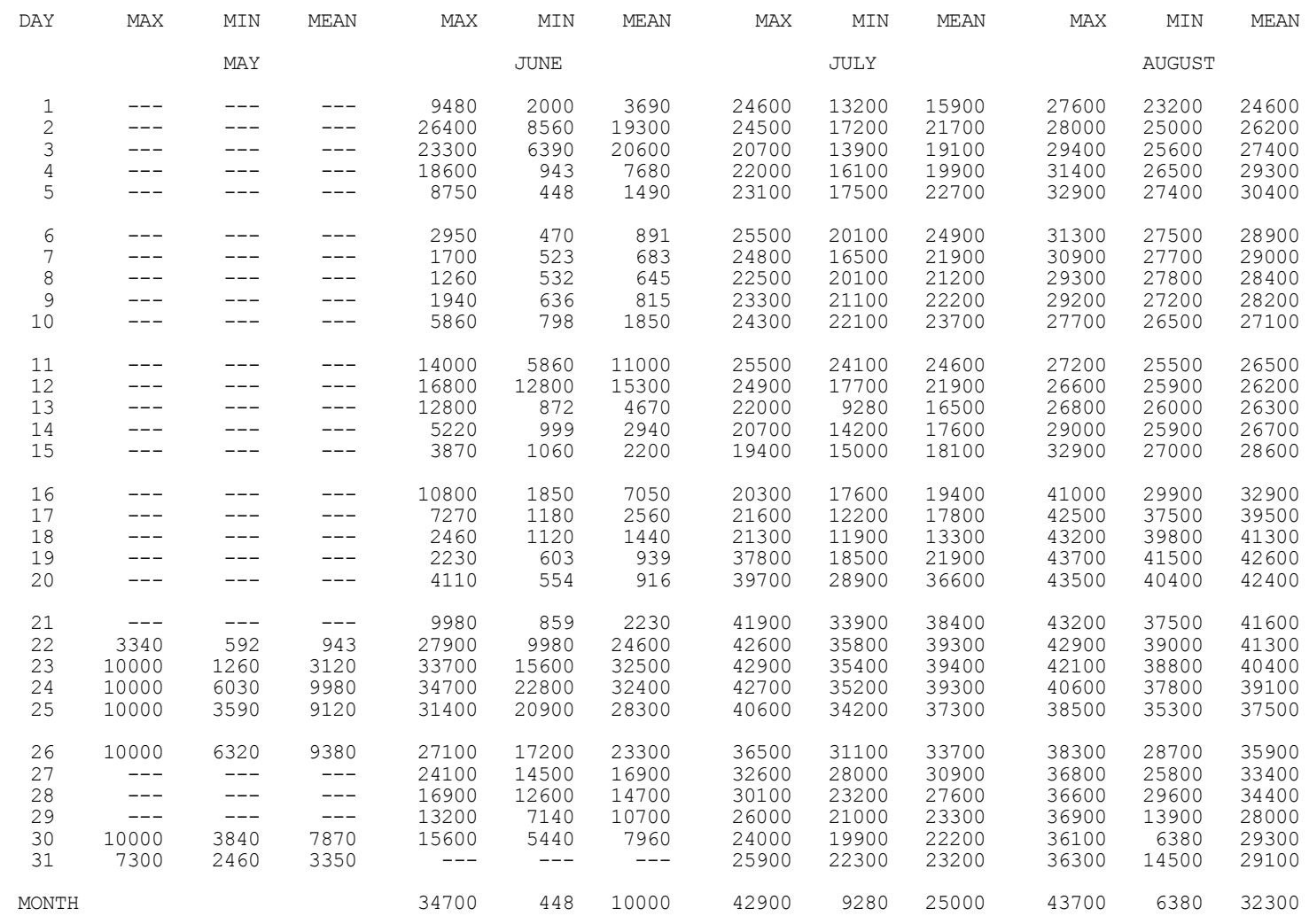

\begin{tabular}{|c|c|c|c|c|c|c|c|c|c|}
\hline \multirow[t]{2}{*}{ DAY } & MAX & MIN & MEAN & MAX & MIN & MEAN & MAX & MIN & MEAN \\
\hline & \multicolumn{3}{|c|}{ SEPTEMBER } & \multicolumn{3}{|c|}{ OCTOBER } & \multicolumn{3}{|c|}{ NOVEMBER } \\
\hline $\begin{array}{l}1 \\
2 \\
3 \\
4 \\
5\end{array}$ & $\begin{array}{l}36900 \\
36400 \\
34700 \\
34900 \\
36200\end{array}$ & $\begin{array}{l}14800 \\
22300 \\
31900 \\
12600 \\
22700\end{array}$ & $\begin{array}{l}26900 \\
31600 \\
33300 \\
26800 \\
34100\end{array}$ & $\begin{array}{l}37300 \\
34300 \\
36000 \\
38300 \\
38900\end{array}$ & $\begin{array}{l}33600 \\
27800 \\
28500 \\
31600 \\
29000\end{array}$ & $\begin{array}{l}35800 \\
32900 \\
34300 \\
35800 \\
36200\end{array}$ & $\begin{array}{l}35400 \\
33800 \\
36600 \\
36800 \\
38800\end{array}$ & $\begin{array}{l}32700 \\
29300 \\
27100 \\
34100 \\
34100\end{array}$ & $\begin{array}{l}34700 \\
31900 \\
33100 \\
35800 \\
37000\end{array}$ \\
\hline $\begin{array}{r}6 \\
7 \\
8 \\
9 \\
10\end{array}$ & $\begin{array}{l}37200 \\
35600 \\
34200 \\
34300 \\
34600\end{array}$ & $\begin{array}{l}33000 \\
16400 \\
12800 \\
33100 \\
23700\end{array}$ & $\begin{array}{l}34900 \\
30400 \\
25300 \\
33700 \\
33600\end{array}$ & $\begin{array}{l}40700 \\
40900 \\
37500 \\
36000 \\
36800\end{array}$ & $\begin{array}{l}29300 \\
36000 \\
30500 \\
29400 \\
26800\end{array}$ & $\begin{array}{l}35700 \\
38800 \\
33200 \\
33700 \\
33400\end{array}$ & $\begin{array}{c}39300 \\
--- \\
--- \\
--- \\
---\end{array}$ & $\begin{array}{r}34300 \\
--- \\
--- \\
--- \\
---\end{array}$ & $\begin{array}{c}37500 \\
--- \\
--- \\
--- \\
---\end{array}$ \\
\hline $\begin{array}{l}11 \\
12 \\
13 \\
14 \\
15\end{array}$ & $\begin{array}{l}34600 \\
35500 \\
39800 \\
43600 \\
44400\end{array}$ & $\begin{array}{r}7160 \\
33200 \\
34600 \\
38200 \\
40600\end{array}$ & $\begin{array}{l}31500 \\
34000 \\
35700 \\
40200 \\
42900\end{array}$ & $\begin{array}{l}39500 \\
38500 \\
42100 \\
43800 \\
43700\end{array}$ & $\begin{array}{l}32900 \\
30500 \\
32200 \\
32100 \\
30800\end{array}$ & $\begin{array}{l}36700 \\
36600 \\
38800 \\
41000 \\
42000\end{array}$ & $\begin{array}{l}--- \\
--- \\
--- \\
--- \\
---\end{array}$ & $\begin{array}{l}--- \\
--- \\
--- \\
--- \\
---\end{array}$ & $\begin{array}{l}--- \\
--- \\
--- \\
--- \\
---\end{array}$ \\
\hline $\begin{array}{l}16 \\
17 \\
18 \\
19 \\
20\end{array}$ & $\begin{array}{l}44500 \\
44600 \\
44500 \\
44600 \\
44200\end{array}$ & $\begin{array}{l}42400 \\
43100 \\
42900 \\
42100 \\
28300\end{array}$ & $\begin{array}{l}43500 \\
43900 \\
43800 \\
43600 \\
39900\end{array}$ & $\begin{array}{l}42900 \\
42500 \\
41300 \\
39600 \\
39100\end{array}$ & $\begin{array}{l}40100 \\
30000 \\
38300 \\
34600 \\
34700\end{array}$ & $\begin{array}{l}41300 \\
39700 \\
40200 \\
37700 \\
36900\end{array}$ & $\begin{array}{l}--- \\
--- \\
--- \\
--- \\
---\end{array}$ & $\begin{array}{l}--- \\
--- \\
--- \\
--- \\
---\end{array}$ & $\begin{array}{l}--- \\
--- \\
--- \\
--- \\
---\end{array}$ \\
\hline $\begin{array}{l}21 \\
22 \\
23 \\
24 \\
25\end{array}$ & $\begin{array}{l}43200 \\
40800 \\
40600 \\
38900 \\
40500\end{array}$ & $\begin{array}{l}39000 \\
25800 \\
36800 \\
36600 \\
34300\end{array}$ & $\begin{array}{l}41500 \\
39000 \\
38900 \\
38200 \\
38800\end{array}$ & $\begin{array}{l}38400 \\
35100 \\
35400 \\
37600 \\
38700\end{array}$ & $\begin{array}{l}28800 \\
27800 \\
29500 \\
27600 \\
31400\end{array}$ & $\begin{array}{l}34100 \\
33300 \\
32900 \\
34300 \\
36400\end{array}$ & $\begin{array}{l}--- \\
--- \\
--- \\
--- \\
---\end{array}$ & $\begin{array}{l}--- \\
--- \\
--- \\
--- \\
---\end{array}$ & $\begin{array}{l}--- \\
--- \\
--- \\
--- \\
---\end{array}$ \\
\hline $\begin{array}{l}26 \\
27 \\
28 \\
29 \\
30 \\
31\end{array}$ & $\begin{array}{r}39600 \\
38400 \\
38600 \\
39000 \\
38700 \\
----\end{array}$ & $\begin{array}{r}30900 \\
33100 \\
31500 \\
33500 \\
35600 \\
----\end{array}$ & $\begin{array}{r}37200 \\
36200 \\
37800 \\
37400 \\
36600 \\
----\end{array}$ & $\begin{array}{l}42600 \\
37800 \\
38400 \\
38300 \\
36300 \\
35200\end{array}$ & $\begin{array}{l}27800 \\
28000 \\
31900 \\
32500 \\
32800 \\
32600\end{array}$ & $\begin{array}{l}32600 \\
33600 \\
36100 \\
34700 \\
34300 \\
33800\end{array}$ & $\begin{array}{l}--- \\
--- \\
--- \\
--- \\
--- \\
---\end{array}$ & $\begin{array}{l}--- \\
--- \\
--- \\
--- \\
--- \\
---\end{array}$ & $\begin{array}{l}--- \\
--- \\
--- \\
--- \\
--- \\
---\end{array}$ \\
\hline MONTH & 44600 & 7160 & 36400 & 43800 & 26800 & 36000 & & & \\
\hline
\end{tabular}


Table A21. Specific conductance and water temperature data collected May-November 2001 at station 102 (USGS identifier 442517068190501) Northeast Creek near Bar Harbor, ME - Continued.

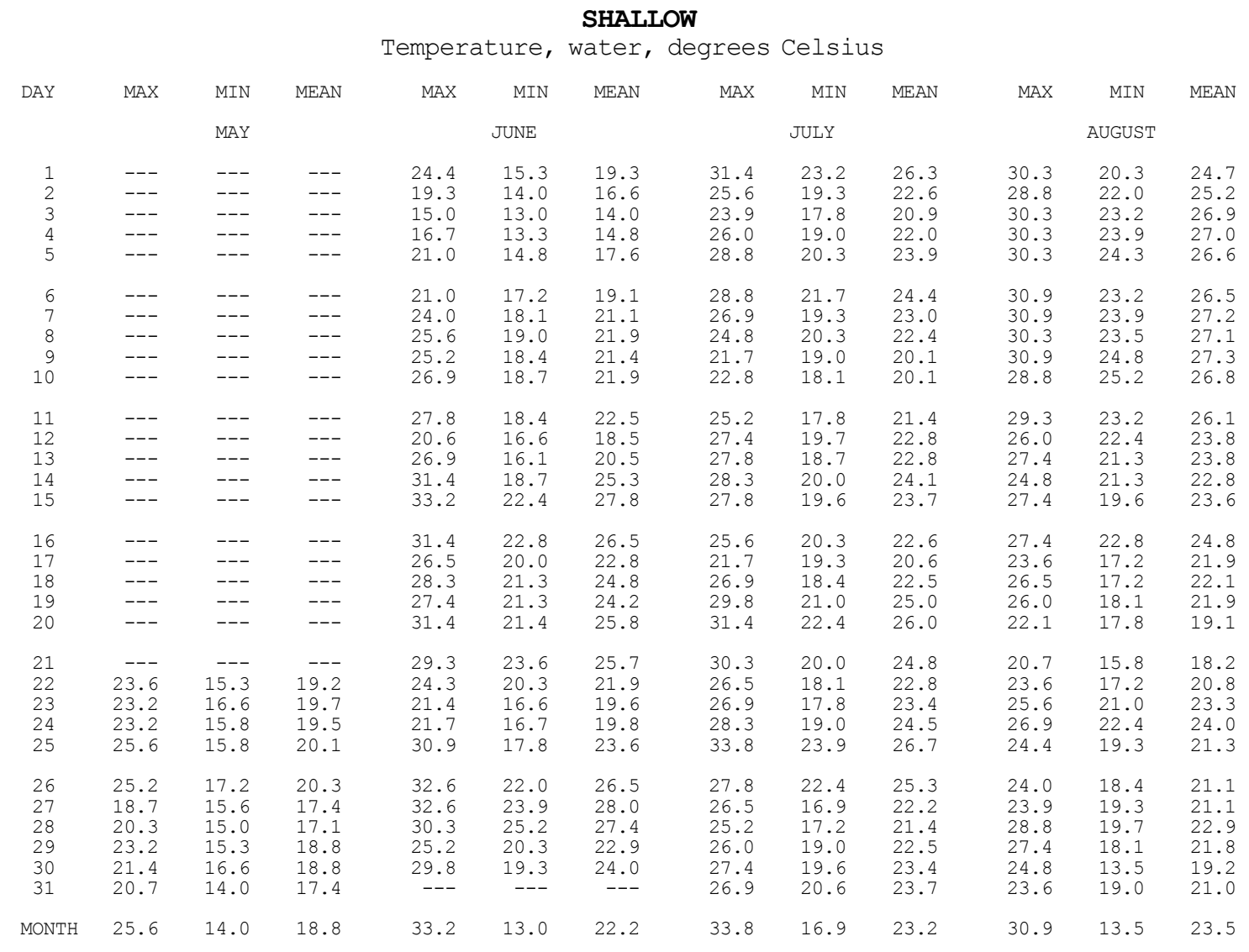

\begin{tabular}{|c|c|c|c|c|c|c|c|c|c|}
\hline \multirow[t]{2}{*}{ DAY } & MAX & MIN & MEAN & MAX & MIN & MEAN & MAX & MIN & MEAN \\
\hline & \multicolumn{3}{|c|}{ SEPTEMBER } & \multicolumn{3}{|c|}{ OCTOBER } & \multicolumn{3}{|c|}{ NOVEMBER } \\
\hline $\begin{array}{l}1 \\
2 \\
3 \\
4 \\
5\end{array}$ & $\begin{array}{l}25.2 \\
23.6 \\
22.1 \\
22.4 \\
24.4\end{array}$ & $\begin{array}{l}15.0 \\
11.9 \\
12.3 \\
15.8 \\
12.6\end{array}$ & $\begin{array}{l}20.6 \\
17.4 \\
17.4 \\
19.0 \\
18.7\end{array}$ & $\begin{array}{l}17.2 \\
20.0 \\
19.4 \\
21.7 \\
21.7\end{array}$ & $\begin{array}{l}10.3 \\
14.0 \\
14.0 \\
15.6 \\
16.1\end{array}$ & $\begin{array}{l}13.8 \\
16.6 \\
16.8 \\
18.1 \\
18.6\end{array}$ & $\begin{array}{r}8.6 \\
13.8 \\
14.5 \\
12.8 \\
12.3\end{array}$ & $\begin{array}{r}6.2 \\
8.6 \\
11.6 \\
10.6 \\
9.9\end{array}$ & $\begin{array}{r}7.2 \\
11.5 \\
13.2 \\
11.8 \\
11.0\end{array}$ \\
\hline $\begin{array}{r}6 \\
7 \\
8 \\
9 \\
10\end{array}$ & $\begin{array}{l}25.6 \\
26.9 \\
29.8 \\
31.4 \\
25.2\end{array}$ & $\begin{array}{r}9.5 \\
12.1 \\
15.3 \\
16.9 \\
17.5\end{array}$ & $\begin{array}{l}16.7 \\
19.4 \\
21.8 \\
22.6 \\
20.7\end{array}$ & $\begin{array}{l}19.0 \\
16.7 \\
14.5 \\
14.3 \\
13.8\end{array}$ & $\begin{array}{r}14.8 \\
11.4 \\
10.3 \\
7.2 \\
9.7\end{array}$ & $\begin{array}{l}17.3 \\
14.1 \\
12.4 \\
10.8 \\
11.8\end{array}$ & $\begin{array}{c}10.4 \\
--- \\
--- \\
--- \\
---\end{array}$ & $\begin{array}{l}8.4 \\
--- \\
--- \\
--- \\
---\end{array}$ & $\begin{array}{l}9.4 \\
--- \\
--- \\
--- \\
---\end{array}$ \\
\hline $\begin{array}{l}11 \\
12 \\
13 \\
14 \\
15\end{array}$ & $\begin{array}{l}26.5 \\
25.6 \\
23.6 \\
19.7 \\
19.7\end{array}$ & $\begin{array}{l}18.4 \\
18.7 \\
18.1 \\
15.5 \\
13.0\end{array}$ & $\begin{array}{l}22.6 \\
21.6 \\
20.6 \\
18.1 \\
16.2\end{array}$ & $\begin{array}{l}16.9 \\
19.7 \\
17.8 \\
15.0 \\
14.8\end{array}$ & $\begin{array}{r}9.1 \\
11.9 \\
13.8 \\
12.6 \\
13.3\end{array}$ & $\begin{array}{l}12.9 \\
15.5 \\
15.6 \\
13.9 \\
13.9\end{array}$ & $\begin{array}{l}--- \\
--- \\
--- \\
--- \\
---\end{array}$ & $\begin{array}{l}--- \\
--- \\
--- \\
--- \\
---\end{array}$ & $\begin{array}{l}--- \\
--- \\
--- \\
--- \\
---\end{array}$ \\
\hline $\begin{array}{l}16 \\
17 \\
18 \\
19 \\
20\end{array}$ & $\begin{array}{l}19.4 \\
19.7 \\
19.7 \\
18.1 \\
17.5\end{array}$ & $\begin{array}{l}13.7 \\
14.5 \\
14.8 \\
15.3 \\
15.3\end{array}$ & $\begin{array}{l}16.3 \\
16.6 \\
17.0 \\
16.6 \\
16.3\end{array}$ & $\begin{array}{l}15.0 \\
14.5 \\
12.6 \\
10.6 \\
11.9\end{array}$ & $\begin{array}{r}11.6 \\
12.3 \\
9.5 \\
6.7 \\
8.9\end{array}$ & $\begin{array}{r}13.3 \\
13.5 \\
11.0 \\
8.9 \\
10.4\end{array}$ & $\begin{array}{l}--- \\
--- \\
--- \\
--- \\
---\end{array}$ & $\begin{array}{l}--- \\
--- \\
--- \\
--- \\
---\end{array}$ & $\begin{array}{l}--- \\
--- \\
--- \\
--- \\
---\end{array}$ \\
\hline $\begin{array}{l}21 \\
22 \\
23 \\
24 \\
25\end{array}$ & $\begin{array}{l}17.8 \\
21.4 \\
25.2 \\
24.0 \\
20.6\end{array}$ & $\begin{array}{l}15.8 \\
17.5 \\
19.0 \\
18.1 \\
18.1\end{array}$ & $\begin{array}{l}17.0 \\
19.1 \\
21.6 \\
20.9 \\
18.9\end{array}$ & $\begin{array}{l}13.5 \\
15.0 \\
12.6 \\
16.1 \\
15.3\end{array}$ & $\begin{array}{r}8.7 \\
11.0 \\
7.8 \\
11.2 \\
13.5\end{array}$ & $\begin{array}{l}11.4 \\
12.8 \\
10.4 \\
13.4 \\
14.3\end{array}$ & $\begin{array}{l}--- \\
--- \\
--- \\
--- \\
---\end{array}$ & $\begin{array}{l}--- \\
--- \\
--- \\
--- \\
---\end{array}$ & $\begin{array}{l}--- \\
--- \\
--- \\
--- \\
---\end{array}$ \\
\hline $\begin{array}{l}26 \\
27 \\
28 \\
29 \\
30 \\
31\end{array}$ & $\begin{array}{r}23.6 \\
19.0 \\
18.1 \\
18.4 \\
18.1 \\
---\end{array}$ & $\begin{array}{r}18.1 \\
15.8 \\
14.2 \\
11.4 \\
10.1 \\
\end{array}$ & $\begin{array}{r}20.0 \\
17.3 \\
16.1 \\
14.9 \\
14.0 \\
---\end{array}$ & $\begin{array}{r}15.3 \\
12.6 \\
10.2 \\
9.3 \\
9.2 \\
7.6\end{array}$ & $\begin{array}{r}10.8 \\
8.3 \\
6.3 \\
3.9 \\
6.0 \\
2.7\end{array}$ & $\begin{array}{r}12.7 \\
10.4 \\
8.2 \\
6.8 \\
7.5 \\
5.6\end{array}$ & $\begin{array}{l}--- \\
--- \\
--- \\
--- \\
--- \\
---\end{array}$ & $\begin{array}{l}--- \\
--- \\
--- \\
--- \\
--- \\
---\end{array}$ & $\begin{array}{l}--- \\
--- \\
--- \\
--- \\
--- \\
---\end{array}$ \\
\hline MONTH & 31.4 & 9.5 & 18.5 & 21.7 & 2.7 & 12.7 & & & \\
\hline
\end{tabular}


Table A21. Specific conductance and water temperature data collected May-November 2001 at station 102 (USGS identifier 442517068190501) Northeast Creek near Bar Harbor, ME - Continued.

DEEP

Temperature, water, degrees Celsius

\begin{tabular}{|c|c|c|c|c|c|c|c|c|c|c|c|c|}
\hline \multirow{2}{*}{ DAY } & MAX & MIN & MEAN & MAX & MIN & MEAN & MAX & MIN & MEAN & MAX & MIN & MEAN \\
\hline & & MAY & & \multicolumn{3}{|c|}{ JUNE } & \multicolumn{3}{|c|}{ JULY } & \multicolumn{3}{|c|}{ AUGUST } \\
\hline 1 & --- & --- & --- & 24.4 & 15.5 & 18.8 & 28.8 & 25.2 & 26.7 & 30.3 & 21.7 & 25.6 \\
\hline 2 & --- & --- & --- & 20.6 & 15.0 & 19.1 & 26.9 & 22.4 & 25.1 & 30.3 & 23.2 & 26.7 \\
\hline 3 & --- & --- & --- & 15.3 & 14.0 & 14.9 & 24.4 & 22.4 & 23.1 & 30.9 & 25.6 & 28.2 \\
\hline 4 & --- & --- & --- & 16.7 & 13.5 & 14.9 & 24.8 & 22.1 & 23.2 & 30.9 & 27.4 & 28.8 \\
\hline 5 & --- & --- & --- & 20.7 & 14.8 & 17.5 & 26.5 & 22.4 & 24.0 & 30.9 & 27.4 & 28.7 \\
\hline 6 & --- & --- & --- & 21.0 & 17.2 & 19.1 & 26.5 & 23.2 & 24.5 & 31.4 & 24.7 & 27.8 \\
\hline 7 & --- & --- & --- & 23.6 & 18.4 & 20.7 & 26.9 & 22.4 & 24.5 & 30.9 & 25.2 & 28.6 \\
\hline 8 & --- & --- & --- & 25.2 & 19.3 & 21.7 & 26.0 & 23.9 & 24.4 & 30.3 & 25.1 & 27.8 \\
\hline 9 & --- & --- & --- & 23.6 & 18.7 & 20.9 & 24.7 & 22.8 & 23.4 & 30.9 & 25.2 & 27.6 \\
\hline 10 & --- & --- & --- & 23.2 & 19.0 & 20.7 & 24.8 & 23.2 & 23.9 & 28.8 & 25.6 & 27.2 \\
\hline 11 & --- & --- & --- & 24.4 & 19.6 & 21.8 & 26.5 & 23.9 & 25.0 & 29.8 & 23.5 & 26.6 \\
\hline 12 & --- & --- & --- & 22.4 & 18.1 & 20.5 & 27.4 & 24.7 & 25.9 & 26.0 & 22.8 & 24.2 \\
\hline 13 & --- & --- & --- & 26.5 & 16.4 & 19.9 & 28.3 & 21.7 & 25.5 & 27.8 & 21.7 & 24.2 \\
\hline 14 & --- & --- & --- & 29.3 & 19.3 & 22.9 & 28.8 & 23.9 & 25.9 & 25.2 & 21.7 & 23.4 \\
\hline 15 & --- & --- & --- & 32.6 & 23.2 & 27.0 & 27.4 & 23.5 & 25.4 & 27.4 & 21.0 & 24.3 \\
\hline 16 & --- & --- & --- & 28.8 & 24.7 & 26.5 & 26.9 & 23.9 & 25.6 & 27.8 & 23.1 & 25.4 \\
\hline 17 & --- & --- & --- & 27.4 & 23.2 & 25.0 & 26.0 & 22.8 & 24.3 & 24.4 & 17.2 & 23.0 \\
\hline 18 & --- & --- & --- & 27.8 & 21.7 & 24.3 & 26.5 & 23.2 & 24.4 & 26.5 & 17.2 & 21.9 \\
\hline 19 & --- & --- & --- & 27.4 & 21.7 & 24.2 & 28.3 & 22.8 & 25.8 & 26.0 & 18.1 & 22.0 \\
\hline 20 & --- & --- & --- & 29.8 & 21.4 & 25.1 & 26.9 & 22.4 & 24.3 & 22.1 & 17.8 & 19.2 \\
\hline 21 & 38.5 & 6.7 & 16.7 & 28.8 & 23.6 & 25.5 & 27.8 & 20.0 & 23.7 & 20.7 & 15.8 & 18.3 \\
\hline 22 & 23.6 & 15.3 & 19.1 & 24.3 & 20.3 & 21.9 & 26.5 & 18.1 & 22.5 & 23.9 & 17.2 & 20.9 \\
\hline 23 & 22.8 & 16.9 & 19.7 & 20.3 & 16.6 & 17.7 & 26.9 & 17.8 & 23.0 & 26.0 & 21.3 & 23.5 \\
\hline 24 & 22.8 & 16.6 & 19.5 & 18.7 & 16.7 & 17.6 & 27.8 & 19.0 & 24.6 & 26.9 & 22.8 & 24.3 \\
\hline 25 & 25.6 & 16.6 & 20.0 & 21.7 & 17.8 & 19.5 & 29.8 & 23.9 & 26.8 & 24.0 & 20.3 & 22.3 \\
\hline 26 & 25.2 & 17.5 & 20.3 & 25.6 & 21.3 & 23.3 & 27.8 & 23.9 & 25.7 & 25.2 & 19.3 & 22.6 \\
\hline 27 & 19.3 & 16.1 & 17.5 & 29.8 & 23.9 & 26.2 & 27.8 & 22.0 & 24.8 & 24.0 & 20.0 & 21.8 \\
\hline 28 & 19.3 & 15.3 & 16.9 & 28.8 & 25.6 & 27.1 & 25.6 & 21.0 & 24.0 & 26.5 & 22.4 & 24.1 \\
\hline 29 & 23.2 & 15.6 & 18.7 & 26.0 & 22.8 & 24.4 & 26.5 & 21.0 & 24.0 & 27.4 & 23.2 & 24.9 \\
\hline 30 & 21.0 & 16.6 & 18.8 & 29.3 & 19.7 & 24.0 & 28.3 & 21.4 & 25.1 & 26.9 & 20.0 & 23.3 \\
\hline 31 & 20.7 & 14.2 & 17.5 & 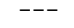 & 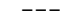 & -- & 26.9 & 22.4 & 24.8 & 24.8 & 20.7 & 22.8 \\
\hline MONTH & 38.5 & 6.7 & 18.6 & 32.6 & 13.5 & 21.8 & 29.8 & 17.8 & 24.6 & 31.4 & 15.8 & 24.5 \\
\hline
\end{tabular}

\begin{tabular}{|c|c|c|c|c|c|c|c|c|c|}
\hline DAY & MAX & MIN & MEAN & MAX & MIN & MEAN & MAX & MIN & MEAN \\
\hline & \multicolumn{3}{|c|}{ SEPTEMBER } & \multicolumn{3}{|c|}{ OCTOBER } & \multicolumn{3}{|c|}{ NOVEMBER } \\
\hline $\begin{array}{l}1 \\
2 \\
3 \\
4 \\
5\end{array}$ & $\begin{array}{l}24.4 \\
24.8 \\
24.0 \\
23.6 \\
24.8\end{array}$ & $\begin{array}{l}21.7 \\
19.0 \\
18.1 \\
18.1 \\
20.6\end{array}$ & $\begin{array}{l}22.7 \\
21.9 \\
21.2 \\
21.2 \\
22.5\end{array}$ & $\begin{array}{l}18.4 \\
19.7 \\
20.0 \\
22.1 \\
21.7\end{array}$ & $\begin{array}{l}14.0 \\
14.5 \\
15.8 \\
15.8 \\
19.3\end{array}$ & $\begin{array}{l}16.6 \\
16.8 \\
17.6 \\
18.9 \\
20.3\end{array}$ & $\begin{array}{r}9.3 \\
14.0 \\
14.3 \\
14.0 \\
13.3\end{array}$ & $\begin{array}{r}7.4 \\
8.6 \\
13.0 \\
12.8 \\
11.0\end{array}$ & $\begin{array}{r}8.7 \\
11.6 \\
13.5 \\
13.5 \\
12.2\end{array}$ \\
\hline $\begin{array}{r}6 \\
7 \\
8 \\
9 \\
10\end{array}$ & $\begin{array}{l}24.8 \\
25.2 \\
26.9 \\
29.3 \\
26.5\end{array}$ & $\begin{array}{l}18.1 \\
18.1 \\
20.0 \\
21.3 \\
22.0\end{array}$ & $\begin{array}{l}21.5 \\
21.6 \\
23.3 \\
24.8 \\
24.1\end{array}$ & $\begin{array}{l}20.0 \\
16.9 \\
15.8 \\
14.3 \\
14.3\end{array}$ & $\begin{array}{r}16.6 \\
14.0 \\
12.6 \\
8.3 \\
10.8\end{array}$ & $\begin{array}{l}17.9 \\
16.1 \\
13.9 \\
11.8 \\
12.6\end{array}$ & $\begin{array}{c}11.2 \\
--- \\
--- \\
--- \\
---\end{array}$ & $\begin{array}{l}9.1 \\
--- \\
--- \\
--- \\
---\end{array}$ & $\begin{array}{c}10.5 \\
--- \\
--- \\
--- \\
--\end{array}$ \\
\hline $\begin{array}{l}11 \\
12 \\
13 \\
14 \\
15\end{array}$ & $\begin{array}{l}26.9 \\
24.8 \\
24.0 \\
22.1 \\
20.3\end{array}$ & $\begin{array}{l}22.1 \\
19.6 \\
19.0 \\
16.4 \\
15.8\end{array}$ & $\begin{array}{l}24.1 \\
22.2 \\
21.4 \\
20.5 \\
17.6\end{array}$ & $\begin{array}{l}16.9 \\
18.4 \\
17.8 \\
15.3 \\
14.8\end{array}$ & $\begin{array}{l}10.6 \\
14.8 \\
15.3 \\
13.3 \\
13.5\end{array}$ & $\begin{array}{l}14.7 \\
16.6 \\
16.6 \\
14.2 \\
14.0\end{array}$ & $\begin{array}{l}--- \\
--- \\
--- \\
--- \\
---\end{array}$ & $\begin{array}{l}--- \\
--- \\
--- \\
--- \\
---\end{array}$ & $\begin{array}{l}--- \\
--- \\
--- \\
--- \\
---\end{array}$ \\
\hline $\begin{array}{l}16 \\
17 \\
18 \\
19 \\
20\end{array}$ & $\begin{array}{l}19.4 \\
19.7 \\
20.0 \\
18.4 \\
17.2\end{array}$ & $\begin{array}{l}14.7 \\
14.8 \\
15.0 \\
15.5 \\
15.3\end{array}$ & $\begin{array}{l}16.6 \\
16.7 \\
17.0 \\
16.8 \\
16.4\end{array}$ & $\begin{array}{l}15.0 \\
14.8 \\
12.6 \\
10.8 \\
12.1\end{array}$ & $\begin{array}{r}11.9 \\
12.3 \\
9.7 \\
7.6 \\
9.1\end{array}$ & $\begin{array}{r}13.5 \\
13.6 \\
11.1 \\
9.3 \\
10.6\end{array}$ & $\begin{array}{l}--- \\
--- \\
--- \\
--- \\
---\end{array}$ & $\begin{array}{l}--- \\
--- \\
--- \\
--- \\
---\end{array}$ & $\begin{array}{l}--- \\
--- \\
--- \\
--- \\
---\end{array}$ \\
\hline $\begin{array}{l}21 \\
22 \\
23 \\
24 \\
25\end{array}$ & $\begin{array}{l}18.1 \\
20.3 \\
24.4 \\
23.6 \\
22.8\end{array}$ & $\begin{array}{l}16.1 \\
17.8 \\
20.0 \\
20.0 \\
19.0\end{array}$ & $\begin{array}{l}17.1 \\
18.8 \\
21.6 \\
22.0 \\
21.1\end{array}$ & $\begin{array}{l}13.8 \\
15.3 \\
13.2 \\
14.3 \\
15.3\end{array}$ & $\begin{array}{r}10.6 \\
11.7 \\
8.9 \\
11.4 \\
14.0\end{array}$ & $\begin{array}{l}12.3 \\
13.4 \\
11.9 \\
12.8 \\
14.5\end{array}$ & $\begin{array}{l}--- \\
--- \\
--- \\
--- \\
---\end{array}$ & $\begin{array}{l}--- \\
--- \\
--- \\
--- \\
---\end{array}$ & $\begin{array}{l}--- \\
--- \\
--- \\
--- \\
---\end{array}$ \\
\hline $\begin{array}{l}26 \\
27 \\
28 \\
29 \\
30 \\
31\end{array}$ & $\begin{array}{r}23.2 \\
22.0 \\
21.3 \\
21.0 \\
19.7 \\
---\end{array}$ & $\begin{array}{r}19.3 \\
19.0 \\
19.3 \\
16.9 \\
14.5 \\
---\end{array}$ & $\begin{array}{r}20.9 \\
20.5 \\
20.5 \\
19.0 \\
17.6 \\
---\end{array}$ & $\begin{array}{r}15.3 \\
13.2 \\
11.0 \\
9.8 \\
9.6 \\
7.8\end{array}$ & $\begin{array}{r}11.9 \\
8.9 \\
7.2 \\
5.0 \\
7.0 \\
4.2\end{array}$ & $\begin{array}{r}14.4 \\
11.9 \\
9.3 \\
7.9 \\
8.5 \\
6.5\end{array}$ & $\begin{array}{l}--- \\
--- \\
--- \\
--- \\
--- \\
---\end{array}$ & $\begin{array}{l}--- \\
--- \\
--- \\
--- \\
--- \\
---\end{array}$ & $\begin{array}{l}--- \\
--- \\
--- \\
--- \\
--- \\
---\end{array}$ \\
\hline MONTH & 29.3 & 14.5 & 20.4 & 22.1 & 4.2 & 13.6 & & & \\
\hline
\end{tabular}


Table A22. Specific conductance and water temperature data collected May-November 2001 at station 103 (USGS identifier 442509068185301) Northeast Creek near Bar Harbor, ME [Data are presented as minimum, mean and maximum daily values of continuous data at 15-minute intervals. Shallow and deep data were collected at fixed depths located, respectively, about $0.2 \mathrm{~m}$ below the water surface and about $0.2 \mathrm{~m}$ above the stream bottom.]

SHALLOW

Specific conductance, water, microsiemens per centimeter at 25 degrees Celsius

\begin{tabular}{|c|c|c|c|c|c|c|c|c|c|c|c|c|}
\hline \multirow[t]{2}{*}{ DAY } & MAX & MIN & MEAN & MAX & MIN & MEAN & MAX & MIN & MEAN & MAX & MIN & MEAN \\
\hline & \multicolumn{3}{|c|}{ MAY } & \multicolumn{3}{|c|}{ JUNE } & \multicolumn{3}{|c|}{ JULY } & \multicolumn{3}{|c|}{ AUGUST } \\
\hline 1 & --- & --- & --- & 2950 & 986 & 1740 & 10500 & 2360 & 4190 & 22900 & \multirow{5}{*}{\multicolumn{2}{|c|}{$\begin{array}{l}20800 \\
22500 \\
23200 \\
23600 \\
24200\end{array}$}} \\
\hline 2 & --- & --- & --- & 10900 & 1250 & 2760 & 10100 & 4800 & 6470 & 23500 & & \\
\hline 3 & --- & --- & --- & 8300 & 352 & 2380 & 8240 & 3560 & 5400 & 24300 & & \\
\hline 4 & --- & --- & --- & 6330 & 246 & 1190 & 8550 & 3990 & 6400 & 25300 & & \\
\hline 5 & --- & --- & --- & 3000 & 254 & 644 & 10300 & 5120 & 7440 & 26600 & & \\
\hline 6 & --- & --- & --- & 1020 & 305 & 457 & 14700 & 5380 & 8680 & 26200 & 25200 & 25600 \\
\hline 7 & --- & --- & --- & 555 & 368 & 432 & 10400 & 4880 & 7880 & 26500 & 24800 & 25500 \\
\hline 8 & --- & --- & --- & 594 & 392 & 539 & 10400 & 3660 & 7460 & 26000 & 24600 & 25200 \\
\hline 9 & --- & --- & --- & 820 & 533 & 706 & 8870 & 1880 & 6190 & 24900 & 18000 & 23000 \\
\hline 10 & --- & --- & --- & 800 & 685 & 761 & 2510 & 1300 & 1930 & 24800 & 17900 & 23800 \\
\hline 11 & --- & --- & --- & 893 & 709 & 811 & 2620 & 1400 & 2020 & 23700 & 17300 & 21000 \\
\hline 12 & --- & --- & --- & 950 & 771 & 867 & 6410 & 2090 & 3260 & 22500 & 15900 & 20700 \\
\hline 13 & --- & --- & --- & 1220 & 632 & 867 & 5600 & 2140 & 3410 & 22400 & 18600 & 21800 \\
\hline 14 & --- & --- & --- & 983 & 583 & 766 & 3760 & 1140 & 2230 & 23100 & 16000 & 19500 \\
\hline 15 & --- & --- & --- & 1020 & 706 & 827 & 3560 & 1080 & 1750 & 25400 & 15400 & 21200 \\
\hline 16 & --- & --- & --- & 1210 & 757 & 910 & 1800 & 1190 & 1470 & 33400 & 17900 & 25100 \\
\hline 17 & --- & --- & --- & 1230 & 871 & 1030 & 3100 & 1530 & 1840 & 43000 & 30600 & 33000 \\
\hline 18 & --- & --- & --- & 1160 & 732 & 905 & 11300 & 2520 & 3630 & 44300 & 20700 & 40400 \\
\hline 19 & --- & --- & --- & 732 & 405 & 489 & 22900 & 6780 & 9730 & 45000 & 24300 & 41600 \\
\hline 20 & --- & --- & --- & 785 & 407 & 506 & 30300 & 17000 & 22900 & 45600 & 35300 & 43700 \\
\hline 21 & --- & --- & --- & 1120 & 530 & 621 & 37700 & 28300 & 35200 & 44800 & 35300 & 42800 \\
\hline 22 & 1360 & 435 & 629 & 5830 & 833 & 1630 & 43200 & 31200 & 40700 & 43700 & 36900 & 41600 \\
\hline 23 & 2770 & 672 & 1100 & 20100 & 2310 & 11300 & 43100 & 34500 & 41000 & 42100 & 38300 & 40100 \\
\hline 24 & 10000 & 1130 & 2480 & 26500 & 2860 & 19100 & 42200 & 34500 & 39800 & 39400 & 35800 & 37900 \\
\hline 25 & 10000 & 1210 & 4560 & 23000 & 5270 & 14200 & 39300 & 34000 & 37200 & 36200 & 33100 & 34900 \\
\hline 26 & 10000 & 2000 & 7960 & 16700 & 3960 & 10700 & 35100 & 26400 & 31900 & 35200 & 33200 & 34500 \\
\hline 27 & 10000 & 2560 & 5500 & 11400 & 3310 & 7070 & 29500 & 20800 & 25000 & 34900 & 33200 & 34000 \\
\hline 28 & 10000 & 2040 & 6610 & 6020 & 3310 & 4280 & 24700 & 21700 & 23400 & 34300 & 32500 & 33300 \\
\hline 29 & 10000 & 3840 & 8430 & 5390 & 2980 & 4080 & 25100 & 23000 & 23900 & 34000 & 31800 & 32800 \\
\hline 30 & 10000 & 1830 & 3480 & 5110 & 3020 & 4000 & 23900 & 21100 & 22600 & 33000 & 31800 & 32300 \\
\hline 31 & 2350 & 1440 & 1960 & --- & --- & --- & 22700 & 19300 & 21300 & 34300 & 32800 & 33200 \\
\hline MONTH & 10000 & 435 & 4270 & 26500 & 246 & 3220 & 43200 & 1080 & 14700 & 45600 & 15400 & 29900 \\
\hline \multirow[t]{2}{*}{ DAY } & MAX & MIN & MEAN & MAX & MIN & MEAN & MAX & MIN & MEAN & & & \\
\hline & \multicolumn{3}{|c|}{ SEPTEMBER } & & OCTOBER & & & OVEMBER & & & & \\
\hline 1 & 34700 & 32300 & 33900 & --- & --- & --- & 33500 & 29500 & 32600 & & & \\
\hline 2 & 33700 & 30100 & 31200 & --- & --- & --- & 33500 & 31800 & 32500 & & & \\
\hline 3 & 32100 & 30500 & 31200 & --- & --- & --- & 32400 & 28600 & 31900 & & & \\
\hline 4 & 32400 & 30100 & 32000 & --- & --- & --- & 33900 & 26800 & 32400 & & & \\
\hline 5 & 32600 & 31700 & 32100 & --- & --- & --- & 36500 & 25900 & 33500 & & & \\
\hline 6 & 32400 & 31500 & 32000 & --- & --- & --- & 33100 & 29800 & 31900 & & & \\
\hline 7 & 32900 & 31400 & 32100 & --- & --- & --- & --- & --- & -- & & & \\
\hline 8 & 33000 & 31700 & 32300 & --- & --- & --- & --- & --- & --- & & & \\
\hline 9 & 33200 & 31800 & 32400 & --- & --- & --- & --- & --- & --- & & & \\
\hline 10 & 33400 & 26700 & 32700 & --- & --- & --- & --- & --- & --- & & & \\
\hline 11 & 33800 & 32100 & 33000 & 36200 & 34200 & 35000 & --- & --- & --- & & & \\
\hline 12 & 34800 & 31900 & 33500 & 36700 & 34900 & 35500 & --- & --- & --- & & & \\
\hline 13 & 35300 & 33700 & 34300 & 40900 & 35300 & 36500 & --- & --- & --- & & & \\
\hline 14 & 39900 & 34600 & 35400 & 44000 & 36400 & 40500 & --- & --- & --- & & & \\
\hline 15 & 44400 & 37800 & 39200 & 44600 & 40200 & 43500 & --- & --- & --- & & & \\
\hline 16 & 46000 & 42000 & 43700 & 43100 & 39200 & 42000 & --- & --- & --- & & & \\
\hline 17 & 46000 & 43000 & 44800 & 44000 & 39000 & 42300 & --- & --- & --- & & & \\
\hline 18 & 46400 & 42800 & 45300 & 42600 & 38600 & 40700 & --- & --- & --- & & & \\
\hline 19 & 46100 & 41700 & 44800 & 40900 & 36800 & 38500 & --- & --- & --- & & & \\
\hline 20 & 45300 & 39700 & 43400 & 38700 & 35900 & 37400 & --- & --- & --- & & & \\
\hline 21 & 43000 & 39700 & 41700 & 37900 & 35600 & 37000 & --- & --- & --- & & & \\
\hline 22 & 42200 & 37100 & 40400 & 36400 & 32000 & 34800 & --- & --- & --- & & & \\
\hline 23 & 39300 & 36300 & 38000 & 34700 & 32800 & 33700 & --- & --- & --- & & & \\
\hline 24 & 37100 & 26600 & 36000 & 34400 & 33300 & 33800 & --- & --- & --- & & & \\
\hline 25 & 36400 & 33600 & 34700 & 34400 & 31500 & 33500 & --- & --- & --- & & & \\
\hline 26 & 38800 & 33400 & 37000 & 33500 & 25700 & 32700 & --- & --- & --- & & & \\
\hline 27 & 37500 & 23000 & 32800 & 34700 & 28900 & 32500 & --- & --- & --- & & & \\
\hline 28 & 30900 & 29500 & 30200 & 32800 & 23800 & 31700 & --- & --- & --- & & & \\
\hline 29 & 31500 & 30300 & 30800 & 33100 & 21700 & 30600 & --- & --- & --- & & & \\
\hline 30 & 32000 & 30900 & 31200 & 32900 & 20500 & 29200 & --- & --- & --- & & & \\
\hline 31 & . & --- & --- & 32800 & 25400 & 30600 & --- & --- & --- & & & \\
\hline MONTH & 46400 & 23000 & 35700 & 44600 & 20500 & 35800 & & & & & & \\
\hline
\end{tabular}


Table A22. Specific conductance and water temperature data collected May-November 2001 at station 103 (USGS identifier 442509068185301) Northeast Creek near Bar Harbor, ME - Continued.

DEEP

Specific conductance, water, microsiemens per centimeter at 25 degrees Celsius

\begin{tabular}{|c|c|c|c|c|c|c|c|c|c|c|c|c|}
\hline DAY & MAX & MIN & MEAN & MAX & MIN & MEAN & MAX & MIN & MEAN & MAX & MIN & MEAN \\
\hline & & MAY & & & JUNE & & \multicolumn{3}{|c|}{ JULY } & \multicolumn{3}{|c|}{ AUGUST } \\
\hline $\begin{array}{l}1 \\
2 \\
3 \\
4 \\
5\end{array}$ & $\begin{array}{l}--- \\
--- \\
--- \\
--- \\
---\end{array}$ & $\begin{array}{l}--- \\
--- \\
--- \\
--- \\
---\end{array}$ & $\begin{array}{l}--- \\
--- \\
--- \\
--- \\
---\end{array}$ & $\begin{array}{r}4230 \\
16400 \\
13600 \\
9580 \\
3330\end{array}$ & $\begin{array}{r}2080 \\
1680 \\
3900 \\
1440 \\
293\end{array}$ & $\begin{array}{r}3080 \\
4020 \\
11100 \\
6300 \\
812\end{array}$ & $\begin{array}{l}13500 \\
14600 \\
13800 \\
13800 \\
15700\end{array}$ & $\begin{array}{r}4150 \\
5420 \\
7490 \\
7700 \\
11600\end{array}$ & $\begin{array}{r}6340 \\
9420 \\
10700 \\
11300 \\
13500\end{array}$ & $\begin{array}{l}25000 \\
23900 \\
24000 \\
25300 \\
26600\end{array}$ & $\begin{array}{l}20900 \\
22100 \\
23000 \\
23400 \\
24000\end{array}$ & $\begin{array}{l}22200 \\
22800 \\
23600 \\
24400 \\
26000\end{array}$ \\
\hline $\begin{array}{r}6 \\
7 \\
8 \\
9 \\
10\end{array}$ & $\begin{array}{l}--- \\
--- \\
--- \\
--- \\
--\end{array}$ & $\begin{array}{l}--- \\
--- \\
--- \\
--- \\
--\end{array}$ & $\begin{array}{l}--- \\
--- \\
--- \\
-- \\
--\end{array}$ & $\begin{array}{r}1200 \\
685 \\
1140 \\
1070 \\
1210\end{array}$ & $\begin{array}{l}315 \\
383 \\
502 \\
670 \\
763\end{array}$ & $\begin{array}{l}488 \\
468 \\
608 \\
803 \\
878\end{array}$ & $\begin{array}{l}16600 \\
19100 \\
18900 \\
18300 \\
15200\end{array}$ & $\begin{array}{r}13400 \\
14500 \\
17000 \\
15000 \\
8580\end{array}$ & $\begin{array}{l}15400 \\
17600 \\
18000 \\
17500 \\
11700\end{array}$ & $\begin{array}{l}26400 \\
26000 \\
26000 \\
25600 \\
25500\end{array}$ & $\begin{array}{l}24900 \\
24800 \\
24600 \\
24500 \\
24600\end{array}$ & $\begin{array}{l}25500 \\
25400 \\
25200 \\
24900 \\
25000\end{array}$ \\
\hline $\begin{array}{l}11 \\
12 \\
13 \\
14 \\
15\end{array}$ & $\begin{array}{l}--- \\
--- \\
--- \\
--- \\
--\end{array}$ & $\begin{array}{l}--- \\
--- \\
--- \\
--- \\
--\end{array}$ & $\begin{array}{l}--- \\
--- \\
--- \\
-- \\
--\end{array}$ & $\begin{array}{l}1100 \\
1140 \\
1590 \\
1600 \\
1580\end{array}$ & $\begin{array}{r}721 \\
795 \\
955 \\
988 \\
1010\end{array}$ & $\begin{array}{r}902 \\
985 \\
1280 \\
1390 \\
1350\end{array}$ & $\begin{array}{l}15900 \\
17000 \\
16500 \\
16500 \\
13800\end{array}$ & $\begin{array}{r}8890 \\
14800 \\
13800 \\
4350 \\
8110\end{array}$ & $\begin{array}{l}12800 \\
15900 \\
15100 \\
13700 \\
11400\end{array}$ & $\begin{array}{l}26700 \\
26100 \\
25700 \\
25400 \\
25700\end{array}$ & $\begin{array}{l}25000 \\
25300 \\
24500 \\
22900 \\
22500\end{array}$ & $\begin{array}{l}26100 \\
25600 \\
25100 \\
24700 \\
23300\end{array}$ \\
\hline $\begin{array}{l}16 \\
17 \\
18 \\
19 \\
20\end{array}$ & $\begin{array}{l}--- \\
--- \\
--- \\
--- \\
--\end{array}$ & $\begin{array}{l}--- \\
--- \\
--- \\
-- \\
--\end{array}$ & $\begin{array}{l}--- \\
--- \\
--- \\
-- \\
--\end{array}$ & $\begin{array}{r}1400 \\
1260 \\
1490 \\
735 \\
747\end{array}$ & $\begin{array}{l}749 \\
927 \\
735 \\
396 \\
411\end{array}$ & $\begin{array}{r}1090 \\
1090 \\
1090 \\
492 \\
514\end{array}$ & $\begin{array}{l}12300 \\
11600 \\
11400 \\
22900 \\
29800\end{array}$ & $\begin{array}{r}6720 \\
2890 \\
3590 \\
9460 \\
22500\end{array}$ & $\begin{array}{r}11200 \\
8170 \\
6840 \\
11900 \\
25000\end{array}$ & $\begin{array}{l}33000 \\
42200 \\
43500 \\
44500 \\
44800\end{array}$ & $\begin{array}{l}25400 \\
18200 \\
25600 \\
40600 \\
36900\end{array}$ & $\begin{array}{l}26300 \\
32300 \\
40300 \\
43000 \\
43500\end{array}$ \\
\hline $\begin{array}{l}21 \\
22 \\
23 \\
24 \\
25\end{array}$ & $\begin{array}{r}--- \\
2520 \\
3430 \\
--- \\
--\end{array}$ & $\begin{array}{l}--- \\
435 \\
755 \\
--- \\
---\end{array}$ & $\begin{array}{r}--- \\
813 \\
1570 \\
--- \\
---\end{array}$ & $\begin{array}{r}1050 \\
7540 \\
23700 \\
26800 \\
23400\end{array}$ & $\begin{array}{r}518 \\
1050 \\
4600 \\
17700 \\
18000\end{array}$ & $\begin{array}{r}617 \\
5910 \\
20800 \\
24400 \\
22500\end{array}$ & $\begin{array}{l}38500 \\
43300 \\
43600 \\
42400 \\
39400\end{array}$ & $\begin{array}{l}29800 \\
31700 \\
36800 \\
37300 \\
36000\end{array}$ & $\begin{array}{l}37000 \\
40900 \\
41600 \\
40300 \\
38000\end{array}$ & $\begin{array}{l}44400 \\
43500 \\
41400 \\
39800 \\
37300\end{array}$ & $\begin{array}{l}40800 \\
39600 \\
27300 \\
18600 \\
30400\end{array}$ & $\begin{array}{l}43300 \\
41900 \\
39400 \\
35000 \\
35900\end{array}$ \\
\hline $\begin{array}{l}26 \\
27 \\
28 \\
29 \\
30 \\
31\end{array}$ & $\begin{array}{r}--- \\
--- \\
--- \\
--- \\
--- \\
10000\end{array}$ & $\begin{array}{c}--- \\
--- \\
--- \\
--- \\
--- \\
1760\end{array}$ & $\begin{array}{r}--- \\
--- \\
--- \\
--- \\
--- \\
3260\end{array}$ & $\begin{array}{r}22800 \\
17100 \\
12600 \\
8090 \\
9450 \\
----\end{array}$ & $\begin{array}{r}16500 \\
11500 \\
3840 \\
4140 \\
4800 \\
----\end{array}$ & $\begin{array}{r}17900 \\
15400 \\
7760 \\
5580 \\
6770 \\
----\end{array}$ & $\begin{array}{l}37700 \\
29800 \\
30500 \\
29100 \\
28500 \\
26100\end{array}$ & $\begin{array}{l}29500 \\
25600 \\
28000 \\
26200 \\
22300 \\
21300\end{array}$ & $\begin{array}{l}33900 \\
28900 \\
28800 \\
27900 \\
25500 \\
24300\end{array}$ & $\begin{array}{l}36100 \\
35700 \\
34300 \\
34200 \\
34000 \\
33900\end{array}$ & $\begin{array}{l}33600 \\
33700 \\
33300 \\
32500 \\
31300 \\
32300\end{array}$ & $\begin{array}{l}34800 \\
34500 \\
33800 \\
33200 \\
32700 \\
32900\end{array}$ \\
\hline MONTH & & & & 26800 & 293 & 5550 & 43600 & 2890 & 20300 & 44800 & 18200 & 30700 \\
\hline \multirow[t]{2}{*}{ DAY } & MAX & MIN & MEAN & MAX & MIN & MEAN & MAX & MIN & MEAN & & & \\
\hline & \multicolumn{3}{|c|}{ SEPTEMBER } & \multicolumn{3}{|c|}{ OCTOBER } & \multicolumn{3}{|c|}{ NOVEMBER } & & & \\
\hline $\begin{array}{l}1 \\
2 \\
3 \\
4 \\
5\end{array}$ & $\begin{array}{l}34200 \\
34400 \\
34300 \\
33200 \\
32300\end{array}$ & $\begin{array}{l}33400 \\
32900 \\
30600 \\
31300 \\
31300\end{array}$ & $\begin{array}{l}33700 \\
33700 \\
32100 \\
31800 \\
31800\end{array}$ & $\begin{array}{l}--- \\
--- \\
--- \\
-- \\
---\end{array}$ & $\begin{array}{l}--- \\
--- \\
--- \\
--- \\
---\end{array}$ & $\begin{array}{l}--- \\
--- \\
--- \\
--- \\
---\end{array}$ & $\begin{array}{l}33700 \\
33700 \\
33300 \\
34000 \\
36600\end{array}$ & $\begin{array}{l}32200 \\
32300 \\
32000 \\
32000 \\
31600\end{array}$ & $\begin{array}{l}33100 \\
33200 \\
32500 \\
33100 \\
34500\end{array}$ & & & \\
\hline $\begin{array}{r}6 \\
7 \\
8 \\
9 \\
10\end{array}$ & $\begin{array}{l}32100 \\
32000 \\
32400 \\
32800 \\
33000\end{array}$ & $\begin{array}{l}31000 \\
30700 \\
31100 \\
31400 \\
32000\end{array}$ & $\begin{array}{l}31600 \\
31600 \\
31800 \\
32100 \\
32500\end{array}$ & $\begin{array}{l}--- \\
--- \\
--- \\
--- \\
--\end{array}$ & $\begin{array}{l}--- \\
--- \\
--- \\
--- \\
---\end{array}$ & $\begin{array}{l}--- \\
--- \\
--- \\
--- \\
--\end{array}$ & $\begin{array}{r}36900 \\
--- \\
--- \\
--- \\
---\end{array}$ & $\begin{array}{r}31900 \\
--- \\
--- \\
--- \\
---\end{array}$ & $\begin{array}{r}33700 \\
--- \\
--- \\
--- \\
---\end{array}$ & & & \\
\hline $\begin{array}{l}11 \\
12 \\
13 \\
14 \\
15\end{array}$ & $\begin{array}{l}33100 \\
34100 \\
34800 \\
39200 \\
43800\end{array}$ & $\begin{array}{l}32400 \\
32600 \\
33400 \\
34300 \\
37500\end{array}$ & $\begin{array}{l}32700 \\
33100 \\
33900 \\
35100 \\
38900\end{array}$ & $\begin{array}{l}35900 \\
36100 \\
40300 \\
43400 \\
43900\end{array}$ & $\begin{array}{l}34500 \\
34500 \\
35100 \\
39000 \\
41600\end{array}$ & $\begin{array}{l}35100 \\
35300 \\
36300 \\
40500 \\
43300\end{array}$ & $\begin{array}{l}--- \\
--- \\
--- \\
--- \\
---\end{array}$ & $\begin{array}{l}--- \\
--- \\
--- \\
--- \\
---\end{array}$ & $\begin{array}{l}--- \\
--- \\
--- \\
--- \\
---\end{array}$ & & & \\
\hline $\begin{array}{l}16 \\
17 \\
18 \\
19 \\
20\end{array}$ & $\begin{array}{l}45100 \\
45300 \\
45900 \\
45600 \\
45100\end{array}$ & $\begin{array}{l}42000 \\
42700 \\
43200 \\
42800 \\
40900\end{array}$ & $\begin{array}{l}43400 \\
44400 \\
44900 \\
44600 \\
43700\end{array}$ & $\begin{array}{l}43000 \\
44100 \\
42800 \\
42100 \\
40600\end{array}$ & $\begin{array}{l}40000 \\
40300 \\
39800 \\
37800 \\
34700\end{array}$ & $\begin{array}{l}42000 \\
42700 \\
41100 \\
40200 \\
38800\end{array}$ & $\begin{array}{l}--- \\
--- \\
--- \\
--- \\
---\end{array}$ & $\begin{array}{l}--- \\
--- \\
--- \\
--- \\
---\end{array}$ & $\begin{array}{l}--- \\
--- \\
--- \\
--- \\
---\end{array}$ & & & \\
\hline $\begin{array}{l}21 \\
22 \\
23 \\
24 \\
25\end{array}$ & $\begin{array}{l}45100 \\
43100 \\
42200 \\
42400 \\
42800\end{array}$ & $\begin{array}{l}40600 \\
40200 \\
40800 \\
41100 \\
35000\end{array}$ & $\begin{array}{l}42700 \\
42100 \\
41500 \\
41700 \\
39800\end{array}$ & $\begin{array}{l}39600 \\
38700 \\
38700 \\
35100 \\
35500\end{array}$ & $\begin{array}{l}36500 \\
36200 \\
33100 \\
33500 \\
33100\end{array}$ & $\begin{array}{l}38300 \\
37500 \\
36800 \\
34200 \\
34400\end{array}$ & $\begin{array}{l}--- \\
--- \\
--- \\
--- \\
---\end{array}$ & $\begin{array}{l}--- \\
--- \\
--- \\
--- \\
---\end{array}$ & $\begin{array}{l}--- \\
--- \\
--- \\
---\end{array}$ & & & \\
\hline $\begin{array}{l}26 \\
27 \\
28 \\
29 \\
30 \\
31\end{array}$ & $\begin{array}{r}40300 \\
40500 \\
40500 \\
41100 \\
39600 \\
---\end{array}$ & $\begin{array}{r}39100 \\
39400 \\
32600 \\
31700 \\
33300 \\
---\end{array}$ & $\begin{array}{r}39700 \\
40000 \\
37100 \\
37200 \\
36700 \\
----\end{array}$ & $\begin{array}{l}34600 \\
34700 \\
34400 \\
34300 \\
34100 \\
33800\end{array}$ & $\begin{array}{l}32800 \\
32900 \\
32600 \\
32400 \\
32200 \\
32700\end{array}$ & $\begin{array}{l}33700 \\
33800 \\
33500 \\
33300 \\
32900 \\
33100\end{array}$ & $\begin{array}{l}--- \\
--- \\
--- \\
--- \\
--- \\
---\end{array}$ & $\begin{array}{l}--- \\
--- \\
--- \\
--- \\
--- \\
---\end{array}$ & $\begin{array}{l}--- \\
--- \\
--- \\
--- \\
--- \\
---\end{array}$ & & & \\
\hline MONTH & 45900 & 30600 & 37200 & 44100 & 32200 & 37000 & & & & & & \\
\hline
\end{tabular}


Table A22. Specific conductance and water temperature data collected May-November 2001 at station 103 (USGS identifier 442509068185301) Northeast Creek near Bar Harbor, ME - Continued.

\section{SHALLOW}

Temperature, water, degrees Celsius

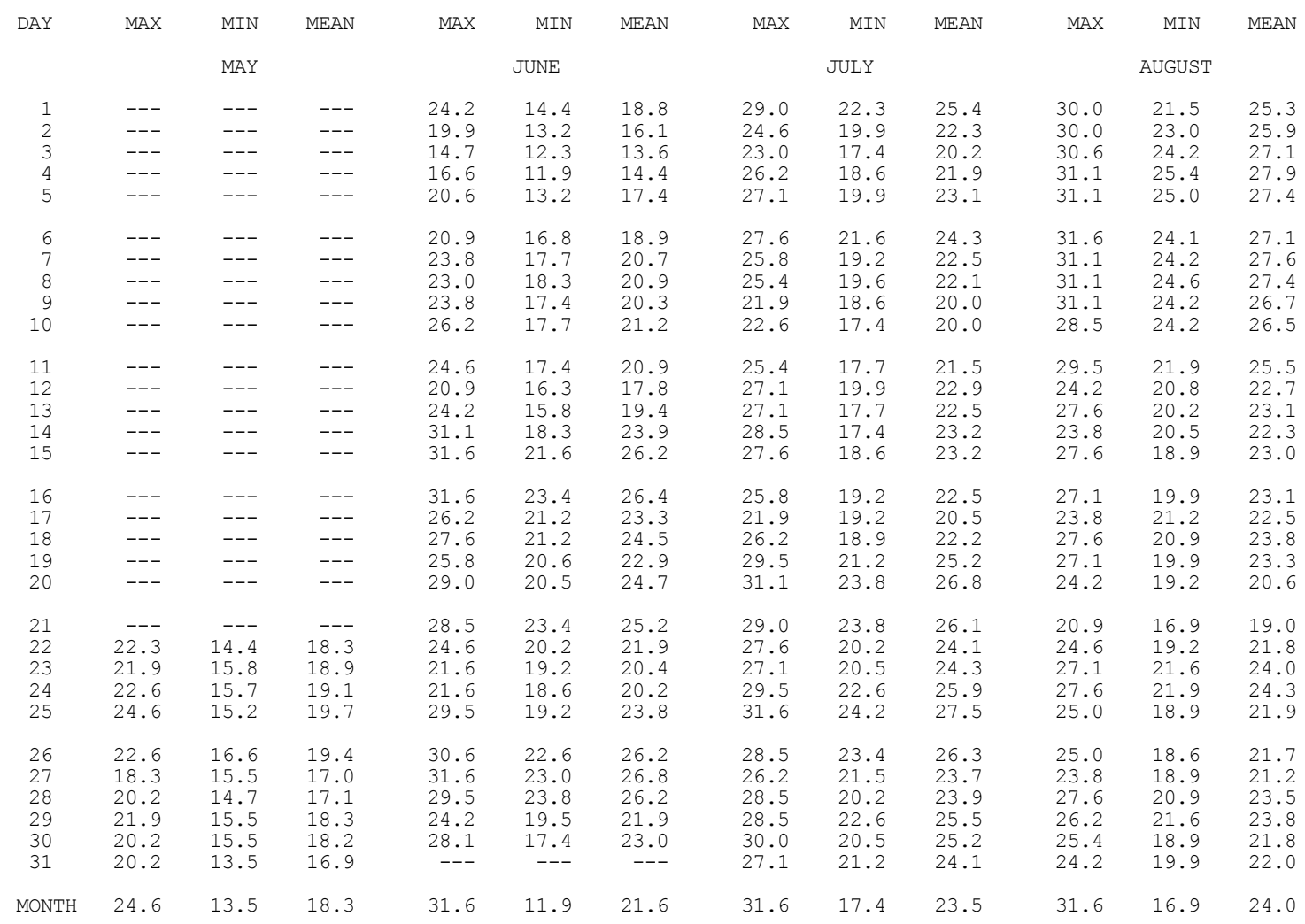

\begin{tabular}{|c|c|c|c|c|c|c|c|c|c|}
\hline \multirow[t]{2}{*}{ DAY } & MAX & MIN & MEAN & MAX & MIN & MEAN & MAX & MIN & MEAN \\
\hline & \multicolumn{3}{|c|}{ SEPTEMBER } & \multicolumn{3}{|c|}{ OCTOBER } & \multicolumn{3}{|c|}{ NOVEMBER } \\
\hline 1 & 23.8 & 18.6 & 22.0 & --- & --- & --- & 9.0 & 6.2 & 7.4 \\
\hline 2 & 24.2 & 15.2 & 19.7 & --- & --- & --- & 13.5 & 8.8 & 11.6 \\
\hline 3 & 22.6 & 16.8 & 19.4 & --- & --- & --- & 14.5 & 13.0 & 13.6 \\
\hline 4 & 22.6 & 17.1 & 20.0 & --- & --- & --- & 14.0 & 12.1 & 13.2 \\
\hline 5 & 23.0 & 19.2 & 20.7 & --- & --- & --- & 13.2 & 8.9 & 11.1 \\
\hline 6 & 21.9 & 16.3 & 19.1 & --- & --- & --- & 9.8 & 7.4 & 8.3 \\
\hline 7 & 25.0 & 17.4 & 20.6 & --- & --- & --- & -- & -- & -- \\
\hline 8 & 26.3 & 18.9 & 22.1 & --- & --- & --- & --- & --- & --- \\
\hline 9 & 28.5 & 20.2 & 23.5 & --- & --- & --- & --- & --- & --- \\
\hline 10 & 25.8 & 21.2 & 23.1 & --- & --- & --- & --- & --- & --- \\
\hline 11 & 25.4 & 21.6 & 23.1 & 17.4 & 9.9 & 13.7 & --- & --- & --- \\
\hline 12 & 24.2 & 18.9 & 21.3 & 20.9 & 12.8 & 16.1 & --- & --- & --- \\
\hline 13 & 23.0 & 18.0 & 20.4 & 17.4 & 13.0 & 15.7 & --- & --- & --- \\
\hline 14 & 20.2 & 16.3 & 17.7 & 16.3 & 11.2 & 14.6 & --- & --- & --- \\
\hline 15 & 20.9 & 12.5 & 16.9 & 15.5 & 14.0 & 14.5 & --- & --- & --- \\
\hline 16 & 20.2 & 14.7 & 17.2 & 15.5 & 12.3 & 14.0 & --- & --- & --- \\
\hline 17 & 20.2 & 15.2 & 17.5 & 14.7 & 11.9 & 13.8 & --- & --- & --- \\
\hline 18 & 20.2 & 16.0 & 18.0 & 12.3 & 9.3 & 10.8 & --- & --- & --- \\
\hline 19 & 19.2 & 16.0 & 17.6 & 11.0 & 8.2 & 9.7 & --- & --- & --- \\
\hline 20 & 17.7 & 15.5 & 16.7 & 13.0 & 9.6 & 11.1 & --- & --- & --- \\
\hline 21 & 18.6 & 16.3 & 17.5 & 16.6 & 10.6 & 13.0 & --- & --- & --- \\
\hline 22 & 21.9 & 18.0 & 19.6 & 15.8 & 11.8 & 13.6 & --- & --- & --- \\
\hline 23 & 26.2 & 19.9 & 22.2 & 13.5 & 8.3 & 11.3 & --- & --- & --- \\
\hline 24 & 24.6 & 18.3 & 21.5 & 16.3 & 11.9 & 13.9 & --- & --- & --- \\
\hline 25 & 21.2 & 16.9 & 19.0 & 15.8 & 13.7 & 14.8 & --- & --- & --- \\
\hline 26 & 23.8 & 19.2 & 21.2 & 15.2 & 12.1 & 13.7 & --- & --- & --- \\
\hline 27 & 21.2 & 16.6 & 19.0 & 13.3 & 9.7 & 11.6 & --- & --- & --- \\
\hline 28 & 17.4 & 13.2 & 15.5 & 11.0 & 6.7 & 9.1 & --- & --- & --- \\
\hline 29 & 18.6 & 11.6 & 14.9 & 10.0 & 4.2 & 7.8 & --- & --- & --- \\
\hline 30 & 18.6 & 10.1 & 14.3 & 9.8 & 6.2 & 8.1 & --- & --- & --- \\
\hline 31 & --- & --- & --- & 7.3 & 3.6 & 6.0 & -- & --- & 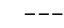 \\
\hline
\end{tabular}


Table A22. Specific conductance and water temperature data collected May-November 2001 at station 103 (USGS identifier 442509068185301) Northeast Creek near Bar Harbor, ME - Continued.

DEEP

Temperature, water, degrees Celsius

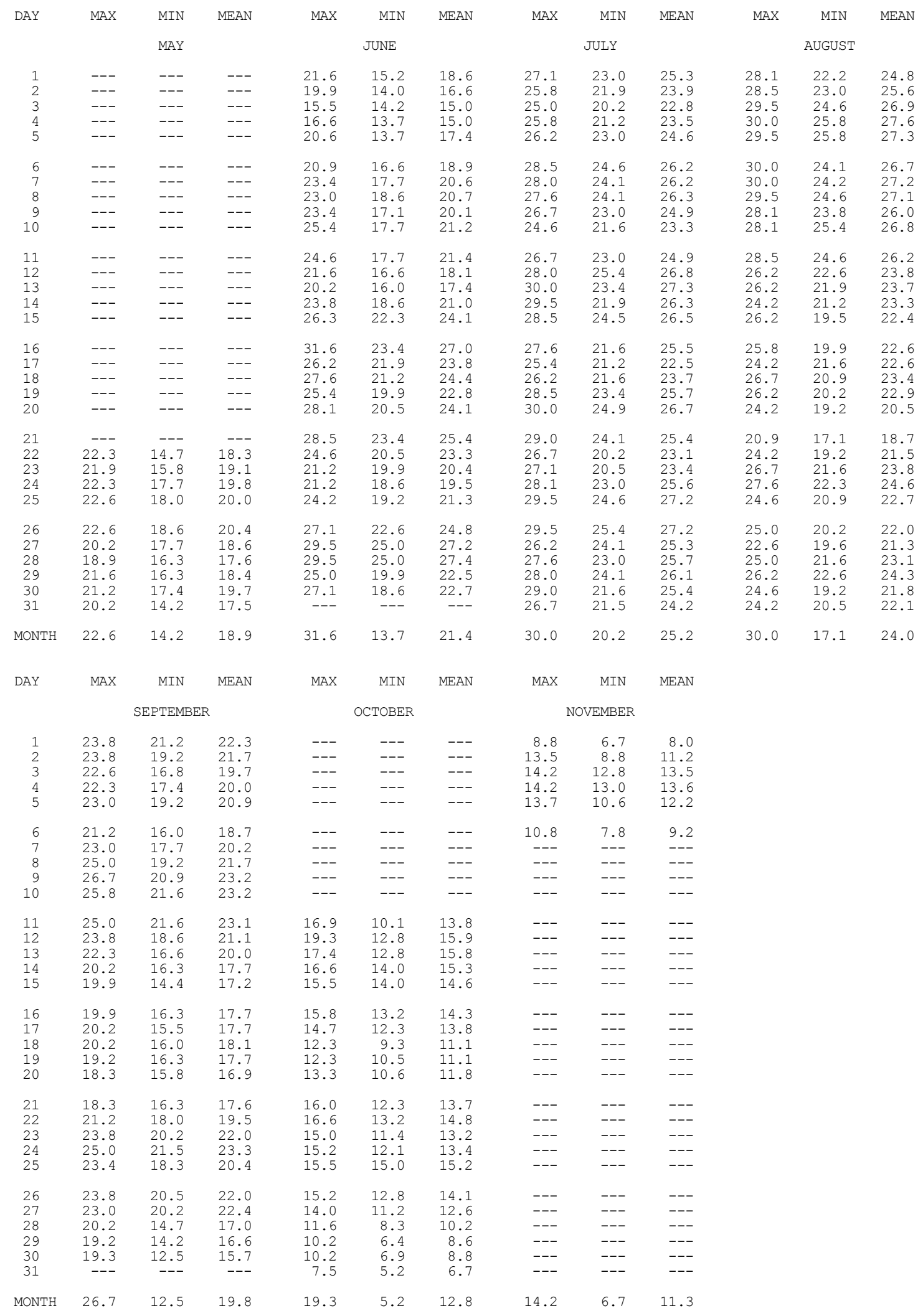


Table A23. Specific conductance and water temperature data collected May-November 2001 at station 104 (USGS identifier 442509068181901) Northeast Creek near Bar Harbor, ME [Data are presented as minimum, mean and maximum daily values of continuous data at 15-minute intervals. Shallow and deep data were collected at fixed depths located, respectively, about $0.2 \mathrm{~m}$ below the water surface and about $0.2 \mathrm{~m}$ above the stream bottom.]

SHALLOW

Specific conductance, water, microsiemens per centimeter at 25 degrees Celsius

\begin{tabular}{|c|c|c|c|c|c|c|c|c|c|c|c|c|}
\hline \multirow[t]{2}{*}{ DAY } & MAX & MIN & MEAN & MAX & MIN & MEAN & MAX & MIN & MEAN & MAX & MIN & MEAN \\
\hline & & MAY & & \multicolumn{3}{|c|}{ JUNE } & \multicolumn{3}{|c|}{ JULY } & \multicolumn{3}{|c|}{ AUGUST } \\
\hline 1 & --- & --- & --- & 867 & 414 & 548 & 2460 & 550 & 859 & 14700 & 8060 & 10700 \\
\hline 2 & --- & --- & --- & 1560 & 388 & 598 & 2240 & 834 & 1300 & 14200 & 10400 & 12800 \\
\hline 3 & --- & --- & --- & 1290 & 187 & 441 & 1420 & 681 & 958 & 14400 & 13100 & 13700 \\
\hline 4 & --- & --- & --- & 775 & 128 & 212 & 1130 & 566 & 787 & 15800 & 11300 & 13300 \\
\hline 5 & --- & --- & --- & 301 & 123 & 146 & 1180 & 560 & 760 & 16900 & 11700 & 13900 \\
\hline 6 & --- & --- & --- & 209 & 121 & 143 & 1570 & 604 & 858 & 15400 & 12500 & 13700 \\
\hline 7 & --- & --- & --- & 203 & 138 & 159 & 1000 & 604 & 764 & 14500 & 13600 & 14000 \\
\hline 8 & --- & --- & --- & 249 & 152 & 194 & 720 & 485 & 581 & 14600 & 14100 & 14300 \\
\hline 9 & --- & --- & --- & 282 & 194 & 231 & 589 & 332 & 447 & 14400 & 13400 & 14000 \\
\hline 10 & --- & --- & --- & 260 & 207 & 232 & 373 & 291 & 322 & 14000 & 13500 & 13800 \\
\hline 11 & --- & --- & --- & 335 & 251 & 290 & 363 & 260 & 309 & 13900 & 13400 & 13600 \\
\hline 12 & --- & --- & --- & 331 & 261 & 295 & 364 & 272 & 316 & 13900 & 13200 & 1350 \\
\hline 13 & --- & --- & --- & 335 & 275 & 301 & 360 & 330 & 347 & 13300 & 12700 & 1300 \\
\hline 14 & --- & --- & --- & 326 & 218 & 261 & 370 & 324 & 349 & 13100 & 12300 & 1270 \\
\hline 15 & --- & --- & --- & 311 & 242 & 267 & 371 & 310 & 337 & 13000 & 11700 & 12600 \\
\hline 16 & --- & --- & --- & 440 & 244 & 320 & 336 & 272 & 298 & 23900 & 12600 & 13800 \\
\hline 17 & --- & --- & --- & 355 & 295 & 324 & 365 & 283 & 314 & 30800 & 21300 & 2410 \\
\hline 18 & --- & --- & --- & 328 & 225 & 268 & 631 & 324 & 360 & 37600 & 24200 & 2820 \\
\hline 19 & --- & --- & --- & 302 & 199 & 225 & 2560 & 439 & 583 & 44100 & 25600 & 3620 \\
\hline 20 & --- & --- & --- & 274 & 165 & 204 & 6960 & 879 & 1610 & 47400 & 16700 & 38000 \\
\hline 21 & --- & --- & --- & 275 & 164 & 213 & 16200 & 3370 & 9080 & 44300 & 28400 & 416 \\
\hline 22 & --- & --- & --- & 481 & 259 & 344 & 33200 & 10300 & 20900 & 42800 & 24700 & 372 \\
\hline 23 & 367 & 193 & 216 & 1550 & 298 & 509 & 38400 & 10300 & 28100 & 41500 & 35700 & 3940 \\
\hline 24 & 1050 & 214 & 317 & 9480 & 298 & 3250 & 37400 & 14800 & 30100 & 38100 & 30300 & 3460 \\
\hline 25 & 2850 & 225 & 595 & 11300 & 476 & 5180 & 32700 & 20600 & 26500 & 33000 & 27800 & 3120 \\
\hline 26 & 3960 & 364 & 1070 & 7730 & 661 & 3120 & 26600 & 16200 & 22400 & 34300 & 29600 & 3130 \\
\hline 27 & 3260 & 498 & 1100 & 3590 & 723 & 1850 & 17600 & 9410 & 13300 & 33800 & 32300 & 3310 \\
\hline 28 & 10000 & 647 & 2230 & 1320 & 508 & 768 & 14700 & 12000 & 13200 & 33400 & 22900 & 3040 \\
\hline 29 & 10000 & 769 & 2350 & 991 & 569 & 743 & 14700 & 11900 & 13400 & 31000 & 15000 & 2540 \\
\hline 30 & 2150 & 551 & 867 & 1110 & 831 & 926 & 14000 & 12200 & 13000 & 31700 & 30100 & 3090 \\
\hline 31 & 744 & 566 & 639 & --- & --- & --- & 14100 & 7740 & 11600 & 33600 & 31100 & 3210 \\
\hline MONTH & 10000 & 193 & 1040 & 11300 & 121 & 752 & 38400 & 260 & 6900 & 47400 & 8060 & 22800 \\
\hline
\end{tabular}

\begin{tabular}{|c|c|c|c|c|c|c|c|c|c|}
\hline \multirow[t]{2}{*}{ DAY } & MAX & MIN & MEAN & MAX & MIN & MEAN & MAX & MIN & MEAN \\
\hline & \multicolumn{3}{|c|}{ SEPTEMBER } & \multicolumn{3}{|c|}{ OCTOBER } & \multicolumn{3}{|c|}{ NOVEMBER } \\
\hline 1 & 33000 & 29300 & 31700 & --_ & --- & --- & 34400 & 31000 & 32200 \\
\hline 2 & 31800 & 29700 & 30500 & --- & --- & --- & 33000 & 32200 & 32600 \\
\hline 3 & 32200 & 30900 & 31500 & --- & --- & --- & 32800 & 31700 & 32200 \\
\hline 4 & 32500 & 31400 & 31900 & --- & --- & --- & 32600 & 31800 & 32200 \\
\hline 5 & 32800 & 31900 & 32300 & --- & --- & --- & 33000 & 30500 & 31900 \\
\hline 6 & 32800 & 32000 & 32400 & --- & --- & --- & --- & --- & --- \\
\hline 7 & 32800 & 31600 & 32400 & --- & --- & --- & --- & --- & --- \\
\hline 8 & 33200 & 32100 & 32600 & --- & --- & --- & --- & --- & -- \\
\hline 9 & 33500 & 32200 & 33000 & --- & --- & --- & --- & --- & --- \\
\hline 10 & --- & --- & --- & 36900 & 35900 & 36400 & --- & --- & --- \\
\hline 11 & --- & --- & --- & 36900 & 36100 & 36400 & --- & --- & --- \\
\hline 12 & --- & --- & --- & 36800 & 11300 & 24600 & --- & --- & --- \\
\hline 13 & --- & --- & --- & 38300 & 35800 & 36500 & --- & --- & --- \\
\hline 14 & --- & --- & --- & 43100 & 27100 & 37800 & --- & --- & --- \\
\hline 15 & --- & --- & --- & 44800 & 40200 & 42400 & --- & --- & --- \\
\hline 16 & 44800 & 37800 & 39600 & 44600 & 16700 & 33300 & --- & --- & --- \\
\hline 17 & 47500 & 43200 & 45700 & 44100 & 18400 & 34400 & --- & --- & --- \\
\hline 18 & 49200 & 45200 & 47800 & 43500 & 14800 & 40900 & --- & --- & --- \\
\hline 19 & 49200 & 46200 & 47700 & 40500 & 38000 & 39000 & --- & --- & --- \\
\hline 20 & 47800 & 44700 & 46300 & 38800 & 18700 & 32400 & --- & --- & --- \\
\hline 21 & 45900 & 42700 & 44400 & 38700 & 26800 & 36500 & --- & --- & --- \\
\hline 22 & 43500 & 40100 & 42300 & 36800 & 34400 & 35700 & --- & --- & --- \\
\hline 23 & 41300 & 37300 & 39500 & 35200 & 33500 & 34400 & --- & --- & --- \\
\hline 24 & 40100 & 34200 & 35700 & 34800 & 33800 & 34300 & --- & --- & --- \\
\hline 25 & 34900 & 33300 & 34400 & 34300 & 10200 & 24200 & --- & --- & --- \\
\hline 26 & 38200 & 32800 & 35200 & 34000 & 24700 & 33400 & --- & --- & --- \\
\hline 27 & 38200 & 32300 & 34800 & 34200 & 33600 & 33900 & --- & -- & --- \\
\hline 28 & 33100 & 31200 & 32100 & 34300 & 33700 & 34000 & --- & --- & --- \\
\hline 29 & 32200 & 30300 & 31000 & 34400 & 33800 & 34000 & --- & --- & --- \\
\hline 30 & 32100 & 31200 & 31600 & 34400 & 26700 & 33900 & --- & --- & -- \\
\hline 31 & & & 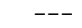 & 34500 & 34000 & 34200 & --- & --- & -1 \\
\hline MONTH & 49200 & 29300 & 36500 & 44800 & 10200 & 34700 & & & \\
\hline
\end{tabular}


Table A23. Specific conductance and water temperature data collected May-November 2001 at station 104 (USGS identifier 442509068181901) Northeast Creek near Bar Harbor, ME - Continued.

DEEP

Specific conductance, water, microsiemens per centimeter at 25 degrees Celsius

\begin{tabular}{|c|c|c|c|c|c|c|c|c|c|c|c|c|}
\hline DAY & MAX & $\begin{array}{l}\text { MIN } \\
\text { MAY }\end{array}$ & MEAN & MAX & $\begin{array}{l}\text { MIN } \\
\text { JUNE }\end{array}$ & MEAN & MAX & $\begin{array}{l}\text { MIN } \\
\text { JULY }\end{array}$ & MEAN & MAX & $\begin{array}{c}\text { MIN } \\
\text { AUGUST }\end{array}$ & MEAN \\
\hline $\begin{array}{l}1 \\
2 \\
3 \\
4 \\
5\end{array}$ & $\begin{array}{l}--- \\
--- \\
--- \\
--- \\
---\end{array}$ & $\begin{array}{l}--- \\
--- \\
--- \\
--- \\
---\end{array}$ & $\begin{array}{l}--- \\
--- \\
--- \\
--- \\
---\end{array}$ & $\begin{array}{l}5250 \\
5000 \\
4090 \\
5070 \\
4810\end{array}$ & $\begin{array}{l}4140 \\
3570 \\
3240 \\
3480 \\
3040\end{array}$ & $\begin{array}{l}4750 \\
4560 \\
3820 \\
4360 \\
4060\end{array}$ & $\begin{array}{l}2790 \\
2710 \\
1200 \\
1000 \\
1300\end{array}$ & $\begin{array}{l}592 \\
845 \\
681 \\
554 \\
603\end{array}$ & $\begin{array}{r}977 \\
1390 \\
1040 \\
824 \\
924\end{array}$ & $\begin{array}{l}21400 \\
21300 \\
20700 \\
17800 \\
19300\end{array}$ & $\begin{array}{l}13100 \\
16600 \\
16700 \\
14600 \\
15000\end{array}$ & $\begin{array}{l}17800 \\
20100 \\
19400 \\
16200 \\
17200\end{array}$ \\
\hline $\begin{array}{r}6 \\
7 \\
8 \\
9 \\
10\end{array}$ & $\begin{array}{l}--- \\
--- \\
--- \\
--- \\
---\end{array}$ & $\begin{array}{l}--- \\
--- \\
--- \\
--- \\
---\end{array}$ & $\begin{array}{l}--- \\
--- \\
--- \\
--- \\
---\end{array}$ & $\begin{array}{r}3040 \\
449 \\
339 \\
348 \\
385\end{array}$ & $\begin{array}{l}127 \\
136 \\
162 \\
189 \\
207\end{array}$ & $\begin{array}{r}1780 \\
202 \\
245 \\
263 \\
270\end{array}$ & $\begin{array}{r}1610 \\
879 \\
795 \\
692 \\
565\end{array}$ & $\begin{array}{l}662 \\
643 \\
624 \\
418 \\
340\end{array}$ & $\begin{array}{r}1030 \\
771 \\
687 \\
544 \\
394\end{array}$ & $\begin{array}{l}17700 \\
17300 \\
14400 \\
13300 \\
13600\end{array}$ & $\begin{array}{l}16200 \\
13800 \\
13300 \\
12900 \\
12600\end{array}$ & $\begin{array}{l}16800 \\
16300 \\
13800 \\
13100 \\
13100\end{array}$ \\
\hline $\begin{array}{l}11 \\
12 \\
13 \\
14 \\
15\end{array}$ & $\begin{array}{l}--- \\
--- \\
--- \\
--- \\
---\end{array}$ & $\begin{array}{l}--- \\
--- \\
--- \\
--- \\
---\end{array}$ & $\begin{array}{l}--- \\
--- \\
--- \\
--- \\
---\end{array}$ & $\begin{array}{l}379 \\
399 \\
506 \\
376 \\
393\end{array}$ & $\begin{array}{l}244 \\
256 \\
315 \\
221 \\
237\end{array}$ & $\begin{array}{l}313 \\
320 \\
360 \\
290 \\
322\end{array}$ & $\begin{array}{l}567 \\
533 \\
441 \\
432 \\
499\end{array}$ & $\begin{array}{l}284 \\
274 \\
317 \\
310 \\
300\end{array}$ & $\begin{array}{l}385 \\
411 \\
367 \\
356 \\
386\end{array}$ & $\begin{array}{l}12900 \\
12600 \\
12100 \\
11800 \\
14100\end{array}$ & $\begin{array}{l}12300 \\
12100 \\
11600 \\
11300 \\
11400\end{array}$ & $\begin{array}{l}12500 \\
12400 \\
11900 \\
11600 \\
11700\end{array}$ \\
\hline $\begin{array}{l}16 \\
17 \\
18 \\
19 \\
20\end{array}$ & $\begin{array}{l}--- \\
--- \\
--- \\
--- \\
---\end{array}$ & $\begin{array}{l}--- \\
--- \\
--- \\
--- \\
---\end{array}$ & $\begin{array}{l}--- \\
--- \\
--- \\
--- \\
---\end{array}$ & $\begin{array}{l}478 \\
467 \\
632 \\
419 \\
332\end{array}$ & $\begin{array}{l}249 \\
291 \\
239 \\
211 \\
177\end{array}$ & $\begin{array}{l}332 \\
352 \\
378 \\
279 \\
261\end{array}$ & $\begin{array}{r}497 \\
354 \\
739 \\
2370 \\
6440\end{array}$ & $\begin{array}{r}274 \\
284 \\
322 \\
428 \\
1040\end{array}$ & $\begin{array}{r}356 \\
329 \\
352 \\
569 \\
2000\end{array}$ & $\begin{array}{l}21800 \\
28200 \\
34300 \\
40400 \\
43100\end{array}$ & $\begin{array}{l}14100 \\
20800 \\
24400 \\
32800 \\
34900\end{array}$ & $\begin{array}{l}15000 \\
22300 \\
28200 \\
38600 \\
41700\end{array}$ \\
\hline $\begin{array}{l}21 \\
22 \\
23 \\
24 \\
25\end{array}$ & $\begin{array}{r}--- \\
--- \\
4870 \\
5270 \\
6240\end{array}$ & $\begin{array}{r}--- \\
--- \\
2690 \\
3220 \\
2850\end{array}$ & $\begin{array}{r}--- \\
--- \\
4190 \\
4490 \\
5340\end{array}$ & $\begin{array}{r}322 \\
497 \\
1670 \\
8830 \\
10600\end{array}$ & $\begin{array}{l}203 \\
243 \\
293 \\
285 \\
680\end{array}$ & $\begin{array}{r}250 \\
344 \\
682 \\
6590 \\
9060\end{array}$ & $\begin{array}{l}15300 \\
30700 \\
35400 \\
34400 \\
31200\end{array}$ & $\begin{array}{r}6440 \\
10000 \\
20500 \\
27600 \\
28800\end{array}$ & $\begin{array}{l}13300 \\
26400 \\
31600 \\
32200 \\
29900\end{array}$ & $\begin{array}{l}41500 \\
40100 \\
38200 \\
37000 \\
34800\end{array}$ & $\begin{array}{l}37400 \\
37400 \\
35800 \\
34500 \\
30500\end{array}$ & $\begin{array}{l}39900 \\
38600 \\
37000 \\
35700 \\
32500\end{array}$ \\
\hline $\begin{array}{l}26 \\
27 \\
28 \\
29 \\
30 \\
31\end{array}$ & $\begin{array}{r}10000 \\
6620 \\
10000 \\
10000 \\
6980 \\
5800\end{array}$ & $\begin{array}{l}4370 \\
4940 \\
6320 \\
5760 \\
4570 \\
4450\end{array}$ & $\begin{array}{l}6790 \\
6290 \\
9390 \\
9540 \\
5810 \\
5320\end{array}$ & $\begin{array}{r}8490 \\
5240 \\
2060 \\
1010 \\
1030 \\
---\end{array}$ & $\begin{array}{r}2860 \\
1770 \\
646 \\
610 \\
817 \\
---\end{array}$ & $\begin{array}{r}6700 \\
3220 \\
1260 \\
780 \\
879 \\
---\end{array}$ & $\begin{array}{l}29800 \\
26600 \\
25800 \\
25100 \\
23600 \\
22300\end{array}$ & $\begin{array}{l}26600 \\
23400 \\
23600 \\
23200 \\
21700 \\
13500\end{array}$ & $\begin{array}{l}28500 \\
24800 \\
24600 \\
24000 \\
22600 \\
19300\end{array}$ & $\begin{array}{l}36800 \\
37000 \\
32700 \\
31300 \\
31000 \\
33400\end{array}$ & $\begin{array}{l}27700 \\
31700 \\
29900 \\
29200 \\
28000 \\
28800\end{array}$ & $\begin{array}{l}33600 \\
33900 \\
31100 \\
30500 \\
29600 \\
32100\end{array}$ \\
\hline
\end{tabular}

\begin{tabular}{|c|c|c|c|c|c|c|c|c|c|}
\hline DAY & MAX & MIN & MEAN & MAX & MIN & MEAN & MAX & MIN & MEAN \\
\hline & \multicolumn{3}{|c|}{ SEPTEMBER } & \multicolumn{3}{|c|}{ OCTOBER } & \multicolumn{3}{|c|}{ NOVEMBER } \\
\hline 1 & 34700 & 30400 & 32300 & --- & --- & --- & 31400 & 11700 & 18500 \\
\hline 2 & 33100 & 29300 & 30700 & --- & --- & --- & 32000 & 23800 & 30300 \\
\hline 3 & 30700 & 17300 & 27500 & --- & --- & --- & 30100 & 9790 & 26400 \\
\hline 4 & 29800 & 28800 & 29200 & --- & --- & --- & 29700 & 21700 & 27600 \\
\hline 5 & 30000 & 29200 & 29500 & --- & --- & --- & 30800 & 14400 & 21100 \\
\hline 6 & 30100 & 22000 & 28700 & --- & --- & --- & --- & --- & --- \\
\hline 7 & 30000 & 15900 & 26600 & --- & --- & --- & --- & --- & --- \\
\hline 8 & 30800 & 20600 & 28600 & --- & --- & --- & --- & --- & --- \\
\hline 9 & 31000 & 18600 & 28600 & --- & --- & --- & --- & --- & --- \\
\hline 10 & --- & --- & --- & 33500 & 15700 & 29200 & --- & --- & --- \\
\hline 11 & --- & --- & --- & 33600 & 16000 & 28000 & --- & --- & --- \\
\hline 12 & --- & --- & --- & 33700 & 32800 & 33200 & --- & --- & --- \\
\hline 13 & --- & --- & --- & 35100 & 11600 & 27200 & --- & --- & --- \\
\hline 14 & --- & --- & --- & 39200 & 17800 & 30300 & --- & --- & --- \\
\hline 15 & --- & --- & --- & 40500 & 14500 & 35600 & --- & --- & --- \\
\hline 16 & 41100 & 18000 & 32200 & 40700 & 38900 & 40200 & --- & --- & --- \\
\hline 17 & 43200 & 40900 & 42200 & 41100 & 37600 & 39700 & --- & --- & --- \\
\hline 18 & 44700 & 41300 & 43900 & 39800 & 37200 & 38100 & --- & --- & --- \\
\hline 19 & 44600 & 42500 & 43700 & 38000 & 25200 & 33300 & --- & --- & --- \\
\hline 20 & 43300 & 40500 & 42300 & 35700 & 34100 & 35100 & --- & --- & --- \\
\hline 21 & 42200 & 39600 & 41000 & 35800 & 25800 & 33700 & --- & --- & --- \\
\hline 22 & 40300 & 38900 & 39800 & 33900 & 25100 & 32300 & --- & --- & --- \\
\hline 23 & 39400 & 38400 & 38900 & 33700 & 16200 & 31200 & --- & --- & --- \\
\hline 24 & 39600 & 37300 & 38300 & 32500 & 23600 & 30600 & --- & --- & --- \\
\hline 25 & 38100 & 34900 & 36800 & 32200 & 15300 & 28500 & --- & --- & --- \\
\hline 26 & 38300 & 35000 & 36400 & 32200 & 17700 & 24900 & --- & --- & --- \\
\hline 27 & 35600 & 34100 & 34800 & 31400 & 14300 & 23000 & --- & --- & --- \\
\hline 28 & 34800 & 33100 & 34000 & 31500 & 11900 & 25100 & --- & --- & --- \\
\hline 29 & 35000 & 30900 & 33100 & 31600 & 17300 & 22700 & -- & --- & --- \\
\hline 30 & 34700 & 19000 & 30000 & 31500 & 15100 & 21600 & --- & --- & --- \\
\hline 31 & --- & --- & --- & 30400 & 11800 & 16800 & --- & --- & --- \\
\hline MONTH & 44700 & 15900 & 34500 & 41100 & 11600 & 30000 & & & \\
\hline
\end{tabular}


Table A23. Specific conductance and water temperature data collected May-November 2001 at station 104 (USGS identifier 442509068181901) Northeast Creek near Bar Harbor, ME - Continued.

\section{SHALLOW}

Temperature, water, degrees Celsius

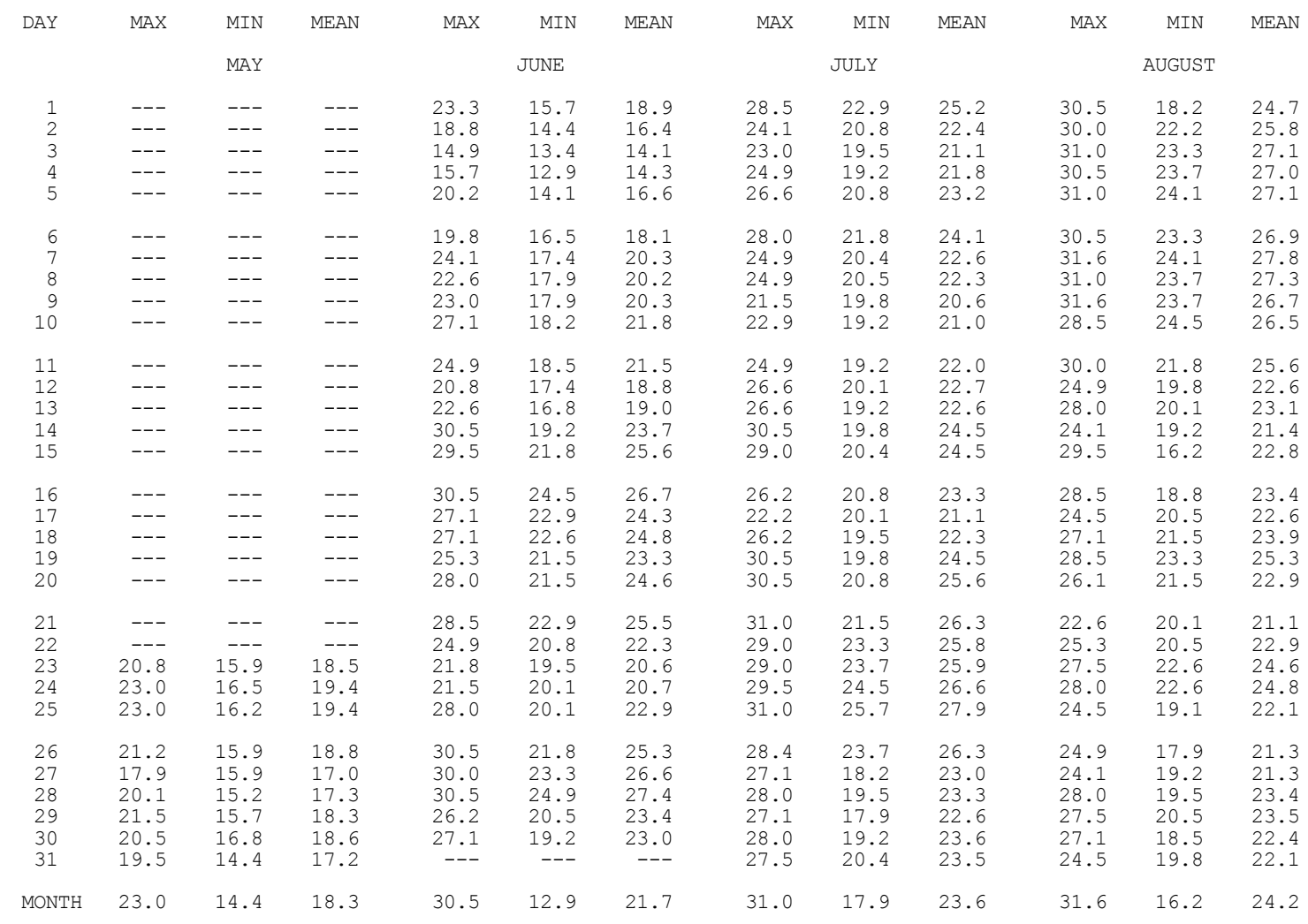

\begin{tabular}{|c|c|c|c|c|c|c|c|c|c|}
\hline DAY & MAX & MIN & MEAN & MAX & MIN & MEAN & MAX & MIN & MEAN \\
\hline & \multicolumn{3}{|c|}{ SEPTEMBER } & \multicolumn{3}{|c|}{ OCTOBER } & \multicolumn{3}{|c|}{ NOVEMBER } \\
\hline 1 & 24.5 & 18.8 & 21.6 & --- & --- & --- & 8.3 & 6.3 & 7.1 \\
\hline 2 & 24.1 & 15.9 & 20.0 & --- & --- & --- & 13.2 & 8.3 & 11.2 \\
\hline 3 & 23.0 & 15.9 & 19.7 & --- & --- & --- & 14.7 & 12.5 & 13.3 \\
\hline 4 & 21.9 & 17.4 & 19.6 & --- & --- & --- & 12.7 & 11.3 & 12.0 \\
\hline 5 & 23.7 & 18.5 & 20.8 & --- & --- & --- & 11.6 & 9.1 & 10.2 \\
\hline 6 & 23.7 & 16.2 & 19.9 & --- & --- & --- & --- & --- & --- \\
\hline 7 & 24.5 & 17.4 & 20.9 & --- & --- & --- & --- & --- & --- \\
\hline 8 & 25.8 & 18.8 & 22.3 & --- & --- & --- & --- & --- & --- \\
\hline 9 & 28.0 & 20.1 & 23.9 & --- & --- & --- & --- & --- & --- \\
\hline 10 & --- & --- & --- & 13.7 & 10.3 & 11.9 & --- & --- & --- \\
\hline 11 & --- & --- & --- & 16.3 & 10.3 & 13.2 & --- & --- & --- \\
\hline 12 & --- & --- & --- & 19.2 & 12.7 & 15.5 & --- & --- & --- \\
\hline 13 & --- & --- & --- & 16.5 & 12.9 & 15.0 & --- & --- & --- \\
\hline 14 & --- & --- & --- & 15.4 & 12.5 & 14.0 & --- & --- & --- \\
\hline 15 & --- & --- & --- & 15.7 & 14.6 & 15.0 & --- & --- & --- \\
\hline 16 & 19.2 & 13.2 & 16.5 & 16.5 & 13.4 & 14.9 & --- & --- & --- \\
\hline 17 & 21.2 & 14.6 & 18.3 & 15.2 & 12.2 & 14.4 & --- & --- & --- \\
\hline 18 & 21.5 & 16.8 & 19.5 & 12.2 & 9.9 & 11.0 & --- & --- & --- \\
\hline 19 & 20.8 & 18.2 & 19.3 & 12.0 & 9.8 & 10.9 & --- & --- & --- \\
\hline 20 & 20.1 & 17.9 & 18.7 & 12.9 & 10.3 & 11.6 & --- & --- & --- \\
\hline 21 & 19.2 & 17.6 & 18.5 & 14.4 & 9.7 & 12.5 & --- & --- & --- \\
\hline 22 & 21.9 & 18.9 & 20.1 & 14.7 & 10.1 & 12.9 & --- & --- & --- \\
\hline 23 & 25.3 & 20.1 & 22.3 & 13.0 & 7.2 & 10.4 & --- & --- & --- \\
\hline 24 & 23.0 & 17.1 & 20.3 & 15.7 & 11.8 & 13.8 & --- & --- & --- \\
\hline 25 & 20.1 & 17.9 & 18.9 & 15.4 & 13.4 & 14.3 & --- & --- & --- \\
\hline 26 & 23.0 & 17.6 & 20.4 & 15.2 & 11.3 & 13.2 & --- & --- & --- \\
\hline 27 & 19.8 & 14.9 & 17.0 & 12.7 & 9.5 & 11.0 & --- & --- & --- \\
\hline 28 & 17.1 & 13.6 & 15.4 & 10.7 & 7.0 & 8.8 & --- & --- & --- \\
\hline 29 & 17.7 & 11.3 & 14.2 & 9.3 & 5.8 & 7.7 & --- & --- & --- \\
\hline 30 & 17.7 & 10.3 & 13.9 & 9.7 & 6.6 & 8.1 & --- & --- & --- \\
\hline 31 & --- & --- & --- & 7.6 & 4.3 & 6.2 & --- & --- & --- \\
\hline MONTH & 28.0 & 10.3 & 19.2 & 19.2 & 4.3 & 12.1 & & & \\
\hline
\end{tabular}


Table A23. Specific conductance and water temperature data collected May-November 2001 at station 104 (USGS identifier 442509068181901) Northeast Creek near Bar Harbor, ME - Continued.

DEEP

Temperature, water, degrees Celsius

\begin{tabular}{|c|c|c|c|c|c|c|c|c|c|}
\hline \multirow[t]{2}{*}{ DAY } & MAX & MIN & MEAN & MAX & MIN & MEAN & MAX & MIN & MEAN \\
\hline & & MAY & & \multicolumn{3}{|c|}{ JUNE } & \multicolumn{3}{|c|}{ JULY } \\
\hline 1 & --- & --- & --- & 20.5 & 14.9 & 17.6 & 25.8 & 22.6 & 24.2 \\
\hline 2 & --- & --- & --- & 18.2 & 14.9 & 16.4 & 24.1 & 20.1 & 22.2 \\
\hline 3 & --- & --- & --- & 14.9 & 13.4 & 14.2 & 21.9 & 19.1 & 20.4 \\
\hline 4 & --- & --- & --- & 15.4 & 13.4 & 14.3 & 23.3 & 18.8 & 20.9 \\
\hline 5 & --- & --- & --- & 18.2 & 13.9 & 15.8 & 24.1 & 20.5 & 22.3 \\
\hline 6 & --- & --- & --- & 18.9 & 15.2 & 17.3 & 24.9 & 21.5 & 23.2 \\
\hline 7 & --- & --- & --- & 22.6 & 16.8 & 19.6 & 24.1 & 19.8 & 22.0 \\
\hline 8 & --- & --- & --- & 21.5 & 17.3 & 19.6 & 22.9 & 20.5 & 21.7 \\
\hline 9 & --- & --- & --- & 21.5 & 17.3 & 19.6 & 21.8 & 19.8 & 20.6 \\
\hline 10 & --- & --- & --- & 23.7 & 17.9 & 20.7 & 21.5 & 19.2 & 20.4 \\
\hline 11 & --- & --- & --- & 22.6 & 18.2 & 20.6 & 22.9 & 19.2 & 21.1 \\
\hline 12 & --- & --- & --- & 21.1 & 17.1 & 18.8 & 23.0 & 20.1 & 21.7 \\
\hline 13 & --- & --- & --- & 20.5 & 16.8 & 18.4 & 23.7 & 19.2 & 21.5 \\
\hline 14 & --- & --- & --- & 24.1 & 18.8 & 21.4 & 24.5 & 19.8 & 22.4 \\
\hline 15 & --- & --- & --- & 26.6 & 21.8 & 24.1 & 24.9 & 20.4 & 22.8 \\
\hline 16 & --- & --- & --- & 30.5 & 24.1 & 26.7 & 24.5 & 21.1 & 22.8 \\
\hline 17 & --- & --- & --- & 25.8 & 22.9 & 24.1 & 22.6 & 20.5 & 21.3 \\
\hline 18 & --- & --- & --- & 27.1 & 21.8 & 24.3 & 23.3 & 19.5 & 21.2 \\
\hline 19 & --- & --- & --- & 24.5 & 20.4 & 22.7 & 24.9 & 19.8 & 22.2 \\
\hline 20 & --- & --- & --- & 26.6 & 20.8 & 23.5 & 25.7 & 20.4 & 23.5 \\
\hline 21 & --- & --- & --- & 27.1 & 22.9 & 25.0 & 29.0 & 25.3 & 26.9 \\
\hline 22 & --- & --- & --- & 24.9 & 20.8 & 22.1 & 28.0 & 24.9 & 26.3 \\
\hline 23 & 20.5 & 15.7 & 18.3 & 21.5 & 19.2 & 20.5 & 28.0 & 23.7 & 25.7 \\
\hline 24 & 21.2 & 15.4 & 18.5 & 21.1 & 20.5 & 20.7 & 29.0 & 24.9 & 26.8 \\
\hline 25 & 21.5 & 17.9 & 19.3 & 23.7 & 20.1 & 21.7 & 29.5 & 26.1 & 27.9 \\
\hline 26 & 21.5 & 17.6 & 19.2 & 26.2 & 22.2 & 24.2 & 29.4 & 28.0 & 28.5 \\
\hline 27 & 18.2 & 17.3 & 17.8 & 28.5 & 23.7 & 25.9 & 28.4 & 26.2 & 27.5 \\
\hline 28 & 18.9 & 17.1 & 18.0 & 30.0 & 24.9 & 26.6 & 29.4 & 27.0 & 28.3 \\
\hline 29 & 20.5 & 16.8 & 18.4 & 25.3 & 20.5 & 23.0 & 28.5 & 26.2 & 27.6 \\
\hline 30 & 20.1 & 16.5 & 18.3 & 25.7 & 18.9 & 21.8 & 29.0 & 26.2 & 27.5 \\
\hline 31 & 19.5 & 13.6 & 16.8 & --- & --- & --- & 27.5 & 22.9 & 25.5 \\
\hline MONTH & 21.5 & 13.6 & 18.3 & 30.5 & 13.4 & 21.0 & 29.5 & 18.8 & 23.8 \\
\hline \multirow[t]{2}{*}{ DAY } & MAX & MIN & MEAN & MAX & MIN & MEAN & MAX & MIN & MEAN \\
\hline & \multicolumn{3}{|c|}{ SEPTEMBER } & \multicolumn{3}{|c|}{ OCTOBER } & \multicolumn{3}{|c|}{ NOVEMBER } \\
\hline 1 & 23.7 & 21.5 & 22.5 & --- & --- & --- & 8.3 & 6.7 & 7.5 \\
\hline 2 & 23.3 & 19.2 & 21.6 & --- & --- & --- & 13.2 & 8.3 & 11.2 \\
\hline 3 & 23.0 & 17.4 & 20.3 & --- & --- & --- & 14.4 & 12.5 & 13.4 \\
\hline 4 & 21.9 & 17.4 & 19.7 & --- & --- & --- & 12.7 & 11.6 & 12.1 \\
\hline 5 & 24.1 & 18.5 & 21.2 & --- & --- & --- & 11.8 & 10.1 & 11.0 \\
\hline 6 & 23.7 & 16.8 & 20.2 & --- & --- & --- & --- & --- & --- \\
\hline 7 & 24.5 & 17.9 & 20.9 & --- & --- & --- & --- & --- & --- \\
\hline 8 & 25.8 & 19.2 & 22.2 & --- & --- & --- & --- & --- & --- \\
\hline 9 & 27.5 & 20.5 & 23.5 & --- & --- & --- & --- & --- & --- \\
\hline 10 & --- & --- & --- & 13.7 & 10.5 & 12.0 & --- & --- & --- \\
\hline 11 & --- & --- & --- & 16.6 & 10.3 & 13.2 & --- & --- & --- \\
\hline 12 & --- & --- & --- & 18.0 & 12.7 & 15.1 & --- & --- & --- \\
\hline 13 & --- & --- & --- & 16.2 & 13.2 & 15.0 & --- & --- & --- \\
\hline 14 & --- & --- & --- & 15.7 & 12.9 & 14.6 & --- & --- & --- \\
\hline 15 & --- & --- & --- & 15.7 & 14.6 & 15.3 & --- & --- & --- \\
\hline 16 & 20.2 & 15.7 & 17.7 & 16.0 & 14.4 & 15.1 & --- & --- & --- \\
\hline 17 & 21.2 & 17.3 & 19.1 & 15.4 & 12.5 & 14.5 & --- & --- & --- \\
\hline 18 & 21.2 & 17.9 & 19.5 & 12.5 & 10.1 & 11.3 & --- & --- & --- \\
\hline 19 & 20.8 & 18.8 & 19.7 & 12.9 & 11.1 & 11.8 & --- & --- & --- \\
\hline 20 & 20.5 & 18.5 & 19.1 & 13.2 & 10.7 & 11.8 & --- & --- & --- \\
\hline 21 & 19.5 & 18.8 & 19.1 & 14.7 & 12.2 & 13.5 & --- & --- & --- \\
\hline 22 & 21.5 & 19.2 & 20.2 & 15.2 & 12.2 & 13.8 & --- & --- & --- \\
\hline 23 & 24.9 & 20.8 & 22.6 & 13.4 & 10.5 & 12.2 & --- & --- & --- \\
\hline 24 & 24.5 & 21.5 & 23.3 & 15.7 & 12.0 & 13.9 & --- & --- & --- \\
\hline 25 & 22.6 & 19.8 & 21.1 & 15.4 & 13.7 & 14.8 & --- & --- & --- \\
\hline 26 & 22.6 & 20.5 & 21.3 & 15.4 & 11.6 & 13.8 & --- & --- & --- \\
\hline 27 & 21.8 & 19.1 & 20.7 & 12.7 & 9.5 & 11.0 & --- & --- & --- \\
\hline 28 & 20.1 & 16.8 & 18.4 & 10.9 & 7.2 & 8.9 & --- & --- & --- \\
\hline 29 & 19.2 & 13.6 & 16.7 & 9.3 & 5.8 & 7.8 & --- & --- & --- \\
\hline 30 & 17.7 & 10.9 & 14.3 & 9.9 & 6.8 & 8.2 & --- & --- & --- \\
\hline 31 & --- & --- & --- & 7.8 & 4.2 & 6.2 & --- & --- & --- \\
\hline MONTH & 27.5 & 10.9 & 20.2 & 18.0 & 4.2 & 12.4 & & & \\
\hline
\end{tabular}

\begin{tabular}{|c|c|c|}
\hline \multirow[t]{2}{*}{ MAX } & MIN & MEAN \\
\hline & AUGUST & \\
\hline $\begin{array}{l}28.5 \\
29.5 \\
29.5 \\
30.5 \\
31.0\end{array}$ & $\begin{array}{l}23.3 \\
24.5 \\
26.6 \\
24.9 \\
26.6\end{array}$ & $\begin{array}{l}26.0 \\
27.4 \\
28.0 \\
27.4 \\
28.3\end{array}$ \\
\hline $\begin{array}{l}30.5 \\
30.5 \\
30.0 \\
29.0 \\
28.5\end{array}$ & $\begin{array}{l}26.6 \\
25.7 \\
24.5 \\
24.1 \\
24.9\end{array}$ & $\begin{array}{l}28.4 \\
28.2 \\
27.2 \\
26.1 \\
26.6\end{array}$ \\
\hline $\begin{array}{l}29.5 \\
24.9 \\
26.6 \\
24.5 \\
26.2\end{array}$ & $\begin{array}{l}22.6 \\
20.4 \\
20.8 \\
20.5 \\
17.9\end{array}$ & $\begin{array}{l}25.5 \\
22.9 \\
23.0 \\
22.4 \\
22.0\end{array}$ \\
\hline $\begin{array}{l}25.8 \\
24.9 \\
26.2 \\
27.5 \\
26.1\end{array}$ & $\begin{array}{l}19.5 \\
21.5 \\
21.8 \\
23.7 \\
23.3\end{array}$ & $\begin{array}{l}23.0 \\
23.0 \\
23.4 \\
25.3 \\
23.8\end{array}$ \\
\hline $\begin{array}{l}23.3 \\
24.9 \\
26.2 \\
28.0 \\
26.1\end{array}$ & $\begin{array}{l}20.5 \\
20.8 \\
22.9 \\
24.9 \\
20.8\end{array}$ & $\begin{array}{l}20.9 \\
22.4 \\
24.6 \\
25.8 \\
23.1\end{array}$ \\
\hline $\begin{array}{l}27.1 \\
26.1 \\
23.7 \\
25.8 \\
24.9 \\
24.9\end{array}$ & $\begin{array}{l}20.1 \\
21.1 \\
21.5 \\
22.9 \\
20.1 \\
20.1\end{array}$ & $\begin{array}{l}23.7 \\
23.1 \\
22.3 \\
24.0 \\
22.7 \\
22.7\end{array}$ \\
\hline 31. & 17.9 & 24.6 \\
\hline
\end{tabular}


Table A24. Specific conductance and water temperature data collected May-November 2001 at station 105 (USGS identifier 442516068175501) Northeast Creek near Bar Harbor, ME [Data are presented as minimum, mean and maximum daily values of continuous data at 15-minute intervals. Shallow and deep data were collected at fixed depths located, respectively, about $0.2 \mathrm{~m}$ below the water surface and about $0.2 \mathrm{~m}$ above the stream bottom.]

\section{SHALLOW}

Specific conductance, water, microsiemens per centimeter at 25 degrees Celsius

\begin{tabular}{|c|c|c|c|c|c|c|c|c|c|}
\hline \multirow[t]{2}{*}{ DAY } & MAX & MIN & MEAN & MAX & MIN & MEAN & MAX & MIN & MEAN \\
\hline & & MAY & & \multicolumn{3}{|c|}{ JUNE } & \multicolumn{3}{|c|}{ JULY } \\
\hline 1 & --- & --- & --- & 144 & 128 & 133 & 161 & 123 & 132 \\
\hline 2 & --- & --- & --- & 196 & 128 & 137 & 147 & 130 & 137 \\
\hline 3 & --- & --- & --- & 132 & 99 & 111 & 149 & 137 & 144 \\
\hline 4 & --- & --- & --- & 100 & 89 & 95 & 145 & 133 & 139 \\
\hline 5 & --- & --- & --- & 93 & 87 & 91 & 142 & 128 & 135 \\
\hline 6 & --- & --- & --- & 96 & 87 & 92 & 147 & 119 & 131 \\
\hline 7 & --- & --- & --- & 709 & 88 & 187 & 134 & 124 & 130 \\
\hline 8 & --- & --- & --- & 114 & 97 & 107 & 139 & 119 & 128 \\
\hline 9 & --- & --- & --- & 138 & 105 & 117 & 133 & 118 & 125 \\
\hline 10 & --- & --- & --- & 137 & 113 & 121 & 129 & 116 & 123 \\
\hline 11 & --- & --- & --- & 157 & 122 & 137 & 124 & 114 & 119 \\
\hline 12 & --- & --- & --- & 149 & 131 & 140 & 122 & 112 & 116 \\
\hline 13 & --- & --- & --- & 169 & 128 & 143 & 124 & 117 & 122 \\
\hline 14 & --- & --- & --- & 159 & 122 & 140 & 136 & 122 & 128 \\
\hline 15 & --- & --- & --- & 145 & 130 & 139 & 136 & 124 & 129 \\
\hline 16 & --- & --- & --- & 151 & 135 & 142 & 131 & 122 & 126 \\
\hline 17 & --- & --- & --- & 162 & 133 & 144 & 130 & 118 & 126 \\
\hline 18 & --- & --- & --- & 161 & 118 & 136 & 135 & 116 & 124 \\
\hline 19 & --- & --- & -- & 121 & 101 & 112 & 170 & 121 & 130 \\
\hline 20 & --- & --- & --- & 119 & 100 & 105 & 211 & 132 & 156 \\
\hline 21 & --- & --- & --- & 112 & 102 & 107 & 410 & 181 & 274 \\
\hline 22 & 174 & 109 & 120 & 130 & 109 & 123 & 2090 & 220 & 865 \\
\hline 23 & 119 & 106 & 113 & 179 & 116 & 137 & 6750 & 403 & 3000 \\
\hline 24 & 130 & 113 & 119 & 179 & 118 & 135 & 16400 & 1440 & 7730 \\
\hline 25 & 140 & 118 & 128 & 167 & 108 & 125 & 14500 & 3660 & 7190 \\
\hline 26 & 145 & 122 & 134 & 162 & 110 & 125 & 11100 & 4660 & 6870 \\
\hline 27 & 153 & 139 & 145 & 134 & 103 & 121 & --- & --- & \\
\hline 28 & 160 & 125 & 141 & 126 & 117 & 122 & --- & --- & --- \\
\hline 29 & 207 & 124 & 140 & 146 & 122 & 136 & --- & --- & \\
\hline 30 & 156 & 124 & 132 & 138 & 126 & 132 & --- & --- & - \\
\hline 31 & 136 & 122 & 130 & & & & --- & --- & - \\
\hline MONTH & 207 & 106 & 130 & 709 & 87 & 126 & 16400 & 112 & 1100 \\
\hline
\end{tabular}

DEEP

Specific conductance, water, microsiemens per centimeter at 25 degrees Celsius

\begin{tabular}{|c|c|c|c|c|c|c|c|c|c|}
\hline \multirow[t]{2}{*}{ DAY } & MAX & MIN & MEAN & MAX & MIN & MEAN & MAX & MIN & MEAN \\
\hline & & MAY & & \multicolumn{3}{|c|}{ JUNE } & \multicolumn{3}{|c|}{ JULY } \\
\hline 1 & --- & --- & --- & 137 & 119 & 127 & 138 & 104 & 113 \\
\hline $\begin{array}{l}2 \\
3\end{array}$ & --- & --- & --- & 188 & 123 & 131 & 142 & 123 & 131 \\
\hline 3 & --- & --- & --- & 129 & 97 & 110 & 140 & 116 & 128 \\
\hline 4 & --- & --- & --- & 97 & 86 & 92 & 139 & 113 & 124 \\
\hline 5 & --- & --- & --- & 90 & 84 & 88 & 138 & 116 & 127 \\
\hline 6 & --- & --- & --- & 93 & 84 & 89 & 133 & 111 & 119 \\
\hline 7 & --- & --- & --- & 105 & 86 & 94 & 127 & 111 & 121 \\
\hline 8 & --- & --- & --- & 106 & 94 & 99 & 125 & 102 & 112 \\
\hline 9 & --- & --- & --- & 112 & 96 & 103 & 115 & 102 & 111 \\
\hline 10 & --- & --- & --- & 129 & 99 & 111 & 123 & 112 & 117 \\
\hline 11 & --- & --- & --- & 132 & 111 & 121 & 118 & 105 & 114 \\
\hline 12 & --- & --- & --- & 144 & 120 & 130 & 113 & 103 & 107 \\
\hline 13 & --- & --- & --- & 149 & 119 & 129 & 116 & 107 & 111 \\
\hline 14 & --- & --- & --- & 136 & 116 & 125 & 125 & 112 & 119 \\
\hline 15 & --- & --- & --- & 137 & 109 & 122 & 123 & 119 & 120 \\
\hline 16 & --- & --- & --- & 138 & 113 & 128 & 121 & 115 & 117 \\
\hline 17 & --- & --- & --- & 133 & 122 & 126 & 120 & 111 & 114 \\
\hline 18 & --- & --- & --- & 153 & 117 & 131 & 122 & 111 & 114 \\
\hline 19 & --- & --- & --- & 117 & 97 & 107 & 140 & 115 & 123 \\
\hline 20 & --- & --- & --- & 104 & 93 & 98 & 190 & 131 & 153 \\
\hline 21 & --- & --- & --- & 123 & 96 & 104 & 377 & 164 & 234 \\
\hline 22 & 145 & 95 & 110 & 122 & 101 & 111 & 3410 & 181 & 1810 \\
\hline 23 & 116 & 100 & 109 & 162 & 102 & 125 & 16300 & 1550 & 11700 \\
\hline 24 & 127 & 101 & 117 & 174 & 100 & 135 & 20600 & 12600 & 18900 \\
\hline 25 & 135 & 108 & 122 & 171 & 110 & 133 & 22200 & 18800 & 21000 \\
\hline 26 & 134 & 109 & 124 & 156 & 110 & 121 & 23600 & 21800 & 22700 \\
\hline 27 & 146 & 112 & 137 & 126 & 104 & 114 & --- & --- & \\
\hline 28 & 151 & 116 & 136 & 120 & 104 & 110 & --- & --- & \\
\hline 29 & 197 & 113 & 131 & 134 & 115 & 125 & --- & --- & --- \\
\hline 30 & 134 & 114 & 124 & 137 & 120 & 126 & --- & --- & -- \\
\hline 31 & 132 & 115 & 126 & --- & -- & -- & --- & --- & --- \\
\hline MONTH & 197 & 95 & 124 & 188 & 84 & 116 & 23600 & 102 & 3030 \\
\hline
\end{tabular}


Table A24. Specific conductance and water temperature data collected May-November 2001 at station 105 (USGS identifier 442516068175501) Northeast Creek near Bar Harbor, ME - Continued.

\begin{tabular}{|c|c|c|c|c|c|c|c|c|c|}
\hline \multirow[b]{3}{*}{ DAY } & \multirow[b]{3}{*}{ MAX } & \multirow[b]{3}{*}{ MIN } & & \multicolumn{6}{|c|}{ SHALLOW } \\
\hline & & & & \multicolumn{2}{|c|}{ Temperature, } & \multirow{2}{*}{$\begin{array}{l}\text { water, } \\
\text { MEAN }\end{array}$} & \multirow[b]{2}{*}{ MAX } & \multicolumn{2}{|c|}{ Celsius } \\
\hline & & & MEAN & MAX & MIN & & & MIN & MEAN \\
\hline & & MAY & & & JUNE & & & JULY & \\
\hline 1 & --- & --- & --- & 22.6 & 14.7 & 18.1 & 29.5 & 22.6 & 25.2 \\
\hline 2 & --- & --- & --- & 18.6 & 13.4 & 15.8 & 24.1 & 20.2 & 22.1 \\
\hline 3 & --- & --- & --- & 13.9 & 12.7 & 13.3 & 23.0 & 18.6 & 20.7 \\
\hline 4 & --- & --- & --- & 15.2 & 13.0 & 13.9 & 25.3 & 18.9 & 21.5 \\
\hline 5 & --- & --- & --- & 18.3 & 13.9 & 16.0 & 27.1 & 20.1 & 23.1 \\
\hline 6 & --- & --- & --- & 18.9 & 15.2 & 17.1 & 26.2 & 21.2 & 23.4 \\
\hline 7 & --- & --- & --- & 21.9 & 16.5 & 18.9 & 26.2 & 19.5 & 22.4 \\
\hline 8 & --- & --- & --- & 23.0 & 16.5 & 19.8 & 24.1 & 19.8 & 21.7 \\
\hline 9 & --- & --- & --- & 23.0 & 16.5 & 19.7 & 20.8 & 18.9 & 19.7 \\
\hline 10 & --- & --- & --- & 24.9 & 16.8 & 20.4 & 21.5 & 18.3 & 19.8 \\
\hline 11 & --- & --- & --- & 24.5 & 16.8 & 20.4 & 23.3 & 18.0 & 20.4 \\
\hline 12 & --- & --- & --- & 19.8 & 16.5 & 17.5 & 26.2 & 18.9 & 21.6 \\
\hline 13 & --- & --- & --- & 25.3 & 16.3 & 19.8 & 27.1 & 18.6 & 22.4 \\
\hline 14 & --- & --- & --- & 29.5 & 18.3 & 23.2 & 28.0 & 19.2 & 23.4 \\
\hline 15 & --- & --- & --- & 32.1 & 21.2 & 26.1 & 27.1 & 19.2 & 22.8 \\
\hline 16 & --- & --- & --- & 29.0 & 23.0 & 25.3 & 25.8 & 19.5 & 21.9 \\
\hline 17 & --- & --- & --- & 27.1 & 21.2 & 23.3 & 20.8 & 18.9 & 19.8 \\
\hline 18 & --- & --- & --- & 24.9 & 21.5 & 22.9 & 24.9 & 18.6 & 21.1 \\
\hline 19 & --- & --- & --- & 25.8 & 19.8 & 22.6 & 28.0 & 18.9 & 23.2 \\
\hline 20 & --- & --- & --- & 30.0 & 21.2 & 24.8 & 29.5 & 20.5 & 24.4 \\
\hline 21 & --- & --- & --- & 27.5 & 22.2 & 24.3 & 30.5 & 21.9 & 24.9 \\
\hline 22 & 21.5 & 14.7 & 17.9 & 23.7 & 19.8 & 21.4 & 28.5 & 22.6 & 25.0 \\
\hline 23 & 21.2 & 15.2 & 18.1 & 20.8 & 19.2 & 20.0 & 29.5 & 23.0 & 25.6 \\
\hline 24 & 21.2 & 14.9 & 18.1 & 21.2 & 19.5 & 20.2 & 30.5 & 24.1 & 26.6 \\
\hline 25 & 22.6 & 15.2 & 18.7 & 28.0 & 19.8 & 22.8 & 30.5 & 24.5 & 27.2 \\
\hline 26 & 22.6 & 15.7 & 19.0 & 29.5 & 21.5 & 24.7 & 27.1 & 22.2 & 24.8 \\
\hline 27 & 18.0 & 15.4 & 16.7 & 31.6 & 23.0 & 26.5 & --- & --- & --- \\
\hline 28 & 18.9 & 14.9 & 16.4 & 28.0 & 23.7 & 25.4 & --- & --- & --- \\
\hline 29 & 21.5 & 15.2 & 18.1 & 23.7 & 20.2 & 21.9 & --- & --- & --- \\
\hline 30 & 19.5 & 16.5 & 18.0 & 29.0 & 18.9 & 23.4 & --- & --- & --- \\
\hline 31 & 19.5 & 14.2 & 16.8 & --- & --- & 列 & --- & --- & --- \\
\hline MONTH & 22.6 & 14.2 & 17.8 & 32.1 & 12.7 & 21.0 & 30.5 & 18.0 & 22.9 \\
\hline
\end{tabular}

DEEP

Temperature, water, degrees Celsius

\begin{tabular}{|c|c|c|c|c|c|c|c|c|c|}
\hline \multirow[t]{2}{*}{ DAY } & MAX & MIN & MEAN & MAX & MIN & MEAN & MAX & MIN & MEAN \\
\hline & & MAY & & \multicolumn{3}{|c|}{ JUNE } & \multicolumn{3}{|c|}{ JULY } \\
\hline 1 & --- & --- & --- & 20.2 & 14.9 & 17.4 & 24.9 & 22.2 & 23.8 \\
\hline 2 & --- & --- & --- & 18.9 & 13.7 & 16.2 & 24.1 & 20.5 & 22.4 \\
\hline 3 & --- & --- & --- & 13.9 & 12.7 & 13.4 & 21.9 & 18.6 & 20.2 \\
\hline 4 & --- & --- & --- & 15.2 & 13.0 & 14.0 & 23.3 & 18.9 & 20.8 \\
\hline 5 & --- & --- & --- & 18.6 & 13.9 & 16.0 & 23.7 & 20.8 & 21.9 \\
\hline 6 & --- & --- & --- & 18.9 & 15.4 & 17.3 & 24.1 & 21.2 & 22.7 \\
\hline 7 & --- & --- & --- & 20.5 & 16.5 & 18.3 & 24.1 & 19.2 & 21.8 \\
\hline 8 & --- & --- & --- & 21.2 & 16.5 & 18.9 & 23.0 & 20.1 & 21.3 \\
\hline 9 & --- & --- & --- & 20.8 & 16.0 & 18.6 & 21.2 & 19.2 & 19.9 \\
\hline 10 & --- & --- & --- & 21.9 & 16.8 & 19.3 & 21.2 & 18.6 & 19.9 \\
\hline 11 & --- & --- & --- & 22.2 & 17.1 & 19.8 & 22.6 & 18.6 & 20.1 \\
\hline 12 & --- & --- & --- & 20.5 & 16.8 & 18.0 & 23.0 & 19.2 & 20.8 \\
\hline 13 & --- & --- & --- & 21.5 & 16.3 & 18.3 & 24.1 & 19.2 & 21.5 \\
\hline 14 & --- & --- & --- & 23.4 & 18.3 & 20.8 & 24.1 & 20.1 & 22.2 \\
\hline 15 & --- & --- & --- & 27.5 & 21.2 & 23.8 & 23.3 & 20.2 & 22.1 \\
\hline 16 & --- & --- & --- & 29.0 & 23.3 & 25.6 & 23.7 & 20.1 & 21.8 \\
\hline 17 & --- & --- & --- & 24.1 & 21.5 & 23.0 & 21.5 & 19.8 & 20.5 \\
\hline 18 & --- & --- & --- & 24.5 & 21.2 & 22.9 & 23.0 & 18.9 & 20.4 \\
\hline 19 & --- & --- & --- & 24.1 & 19.5 & 22.0 & 23.4 & 19.2 & 21.2 \\
\hline 20 & --- & --- & --- & 26.2 & 20.5 & 23.3 & 24.5 & 20.8 & 22.7 \\
\hline 21 & --- & --- & --- & 25.3 & 22.2 & 23.6 & 24.5 & 21.2 & 23.2 \\
\hline 22 & 20.5 & 14.2 & 17.1 & 23.7 & 20.1 & 21.5 & 25.8 & 23.0 & 24.2 \\
\hline 23 & 19.8 & 15.4 & 17.7 & 20.5 & 18.9 & 19.6 & 27.1 & 24.1 & 25.7 \\
\hline 24 & 20.8 & 14.4 & 17.8 & 20.8 & 19.5 & 20.0 & 28.5 & 25.8 & 26.9 \\
\hline 25 & 20.5 & 15.4 & 17.8 & 23.7 & 19.8 & 21.3 & 29.5 & 26.6 & 28.0 \\
\hline 26 & 21.9 & 15.2 & 18.5 & 24.9 & 21.2 & 23.1 & 29.0 & 28.0 & 28.5 \\
\hline 27 & 18.6 & 15.2 & 16.9 & 27.5 & 22.6 & 24.7 & --- & --- & --- \\
\hline 28 & 17.7 & 14.9 & 16.3 & 26.6 & 23.3 & 24.8 & --- & --- & --- \\
\hline 29 & 19.8 & 14.9 & 17.3 & 24.1 & 20.8 & 21.7 & --- & --- & --- \\
\hline 30 & 19.2 & 16.3 & 17.8 & 24.9 & 19.2 & 21.6 & --- & --- & --- \\
\hline 31 & 19.2 & 14.2 & 16.7 & -- & -- & --- & 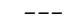 & --- & --- \\
\hline MONTH & 21.9 & 14.2 & 17.4 & 29.0 & 12.7 & 20.3 & 29.5 & 18.6 & 22.5 \\
\hline
\end{tabular}




\section{Appendix 3}

Stage data at stations 101 and 103 
Table A25. Stage data collected May-December 2000 at station 101 (USGS identifier 01022820) Northeast Creek at Route 3 bridge near Bar Harbor, ME [Data are presented as minimum, mean and maximum daily values of continuous data collected at 15-minute intervals.]

GAGE HEIGHT, IN FEET

\begin{tabular}{|c|c|c|c|c|c|c|c|c|c|c|c|c|}
\hline \multirow{2}{*}{ DAY } & MAX & MIN & MEAN & MAX & MIN & MEAN & MAX & MIN & MEAN & MAX & MIN & MEAN \\
\hline & & MAY & & \multicolumn{3}{|c|}{ JUNE } & \multicolumn{3}{|c|}{ JULY } & \multicolumn{3}{|c|}{ AUGUST } \\
\hline 1 & --- & --- & --- & --- & --- & --- & 3.64 & 2.45 & 2.70 & --- & --- & --- \\
\hline 2 & --- & --- & --- & --- & --- & --- & 3.63 & 2.54 & 2.79 & --- & --- & --- \\
\hline 3 & --- & --- & --- & --- & --- & --- & 3.76 & 2.61 & 2.87 & --- & --- & --- \\
\hline 4 & --- & --- & --- & 3.58 & 2.69 & 2.91 & 3.72 & 2.64 & 2.90 & --- & --- & --- \\
\hline 5 & --- & --- & --- & 3.51 & 2.62 & 2.87 & 3.70 & 2.65 & 2.90 & --- & --- & --- \\
\hline 6 & --- & --- & --- & 3.24 & 2.58 & 2.78 & 3.29 & 2.64 & 2.78 & --- & --- & --- \\
\hline 7 & --- & --- & --- & 3.30 & 2.56 & 2.85 & 2.93 & 2.51 & 2.64 & --- & --- & -- \\
\hline 8 & --- & --- & --- & 2.96 & 2.69 & 2.78 & 2.57 & 2.33 & 2.42 & 2.12 & 2.05 & 2.08 \\
\hline 9 & --- & --- & --- & 2.77 & 2.55 & 2.64 & 2.33 & 2.17 & 2.24 & 2.11 & 2.02 & 2.04 \\
\hline 10 & --- & --- & --- & 2.55 & 2.42 & 2.47 & --- & --- & --- & 2.04 & 1.99 & 2.02 \\
\hline 11 & --- & --- & --- & 2.49 & 2.40 & 2.42 & --- & --- & --- & 2.03 & 1.97 & 1.99 \\
\hline 12 & --- & --- & --- & 2.46 & 2.37 & 2.41 & --- & --- & --- & 2.08 & 1.95 & 1.98 \\
\hline 13 & --- & --- & --- & 2.42 & 2.33 & 2.37 & --- & --- & --- & 2.20 & 1.96 & $\begin{array}{l}1.99 \\
\end{array}$ \\
\hline 14 & --- & --- & --- & 2.38 & 2.27 & 2.33 & --- & --- & --- & 2.26 & 1.98 & 2.03 \\
\hline 15 & --- & --- & --- & 2.41 & 2.25 & 2.29 & --- & --- & --- & 2.26 & 2.01 & 2.06 \\
\hline 16 & --- & --- & --- & 2.43 & 2.25 & 2.30 & --- & --- & --- & 2.30 & 2.05 & 2.10 \\
\hline 17 & --- & --- & --- & 2.44 & 2.25 & 2.32 & --- & --- & --- & 2.39 & 2.05 & 2.13 \\
\hline 18 & 2.97 & 2.68 & 2.74 & 2.35 & 2.27 & 2.30 & --- & --- & --- & 2.24 & 2.02 & 2.07 \\
\hline 19 & 2.99 & 2.84 & 2.88 & 2.34 & 2.22 & 2.27 & --- & --- & --- & 2.16 & 2.01 & 2.05 \\
\hline 20 & 2.94 & 2.75 & 2.83 & 2.27 & 2.18 & 2.21 & --- & --- & --- & 2.11 & 2.02 & 2.05 \\
\hline 21 & 2.82 & 2.64 & 2.71 & 2.21 & 2.13 & 2.17 & --- & --- & --- & 2.06 & 2.02 & 2.04 \\
\hline 22 & 2.66 & 2.56 & 2.61 & 2.24 & 2.13 & 2.21 & --- & --- & --- & 2.04 & 1.98 & 2.00 \\
\hline 23 & 2.62 & 2.55 & 2.59 & 2.24 & 2.16 & 2.19 & --- & --- & --- & 2.07 & 1.94 & 1.98 \\
\hline 24 & 2.90 & 2.62 & 2.68 & 2.16 & 2.10 & 2.13 & --- & --- & --- & 2.25 & 1.97 & 2.01 \\
\hline 25 & 3.15 & 2.90 & 3.04 & 2.10 & 2.05 & 2.08 & --- & --- & --- & 2.49 & 2.00 & 2.08 \\
\hline 26 & 3.18 & 3.14 & 3.16 & 2.08 & 2.05 & 2.06 & --- & --- & --- & 2.90 & 2.03 & 2.18 \\
\hline 27 & 3.14 & 3.02 & 3.07 & 2.24 & 2.03 & 2.07 & --- & --- & --- & 3.27 & 2.16 & 2.35 \\
\hline 28 & 3.02 & 2.87 & 2.94 & 2.56 & 2.06 & 2.15 & --- & --- & --- & 3.39 & 2.26 & 2.48 \\
\hline 29 & 2.87 & 2.72 & 2.80 & 3.10 & 2.17 & 2.34 & --- & --- & --- & 3.39 & 2.34 & 2.56 \\
\hline 30 & 2.72 & 2.60 & 2.67 & 3.45 & 2.36 & 2.58 & --- & --- & --- & 3.40 & 2.39 & 2.61 \\
\hline 31 & --- & --- & --- & --- & --- & --- & --- & --- & --- & 3.28 & 2.37 & 2.60 \\
\hline MONTH & 3.18 & 2.55 & 2.82 & 3.58 & 2.03 & 2.39 & 3.76 & 2.17 & 2.69 & 3.40 & 1.94 & 2.15 \\
\hline \multirow[t]{2}{*}{ DAY } & MAX & MIN & MEAN & MAX & MIN & MEAN & MAX & MIN & MEAN & MAX & MIN & MEAN \\
\hline & \multicolumn{3}{|c|}{ SEPTEMBER } & \multicolumn{3}{|c|}{ OCTOBER } & \multicolumn{3}{|c|}{ NOVEMBER } & \multicolumn{3}{|c|}{ DECEMBER } \\
\hline 1 & 3.07 & 2.35 & 2.53 & 2.48 & 2.19 & 2.27 & 3.06 & 2.96 & 3.03 & 3.31 & 3.12 & 3.22 \\
\hline 2 & 2.72 & 2.27 & 2.39 & 2.34 & 2.11 & 2.17 & 3.06 & 2.98 & 3.03 & 3.13 & 2.96 & 3.03 \\
\hline 3 & 2.50 & 2.20 & 2.29 & 2.35 & 2.04 & 2.12 & 2.98 & 2.84 & 2.91 & 2.96 & 2.80 & 2.87 \\
\hline 4 & 2.34 & 2.17 & 2.22 & 2.10 & 2.01 & 2.05 & 2.84 & 2.69 & 2.77 & 2.80 & 2.67 & 2.73 \\
\hline 5 & 2.17 & 2.06 & 2.10 & 2.01 & 1.96 & 1.98 & 2.69 & 2.62 & 2.65 & 2.67 & 2.58 & 2.61 \\
\hline 6 & 2.06 & 2.00 & 2.03 & 2.22 & 1.97 & 2.10 & 2.62 & 2.59 & 2.61 & 2.59 & 2.52 & 2.55 \\
\hline 7 & 2.00 & 1.96 & 1.98 & 2.25 & 2.20 & 2.23 & 2.59 & 2.54 & 2.56 & 2.52 & 2.45 & 2.48 \\
\hline 8 & 1.96 & 1.93 & 1.95 & 2.20 & 2.12 & 2.16 & 2.54 & 2.48 & 2.51 & 2.45 & 2.37 & 2.41 \\
\hline 9 & 1.93 & 1.90 & 1.91 & 2.21 & 2.11 & 2.14 & 2.70 & 2.45 & 2.53 & 2.56 & 2.33 & 2.38 \\
\hline 10 & 1.90 & 1.88 & 1.89 & 2.32 & 2.21 & 2.29 & 3.02 & 2.66 & 2.76 & 2.49 & 2.27 & 2.32 \\
\hline 11 & 1.89 & 1.86 & 1.87 & 2.30 & 2.23 & 2.26 & 3.24 & 2.69 & 2.82 & 3.54 & 2.24 & 2.61 \\
\hline 12 & 2.03 & 1.84 & 1.87 & 2.24 & 2.18 & 2.20 & 3.28 & 2.66 & 2.81 & 4.41 & 2.51 & 2.99 \\
\hline 13 & 2.13 & 1.92 & 1.95 & 2.24 & 2.15 & 2.19 & 3.25 & 2.62 & 2.78 & 3.21 & 2.73 & 2.86 \\
\hline 14 & 2.20 & 1.99 & 2.02 & 2.44 & 2.16 & 2.24 & 3.39 & 2.59 & 2.80 & 3.70 & 2.60 & 2.87 \\
\hline $\begin{array}{l}14 \\
15\end{array}$ & 2.48 & 2.07 & 2.19 & 2.42 & 2.19 & 2.28 & 3.36 & 2.78 & 2.93 & 2.95 & 2.73 & 2.79 \\
\hline 16 & 2.67 & 2.22 & 2.35 & 2.42 & 2.17 & 2.24 & 2.98 & 2.80 & 2.85 & 2.80 & 2.65 & 2.70 \\
\hline 17 & 2.42 & 2.15 & 2.24 & 2.39 & 2.13 & 2.22 & 2.96 & 2.72 & 2.78 & 3.53 & 2.66 & 3.12 \\
\hline 18 & 2.24 & 2.07 & 2.12 & 2.46 & 2.14 & 2.22 & 2.73 & 2.60 & 2.66 & 3.87 & 3.52 & 3.74 \\
\hline 19 & 2.19 & 2.03 & 2.06 & 2.63 & 2.22 & 2.39 & 2.60 & 2.50 & 2.54 & 3.87 & 3.70 & 3.79 \\
\hline 20 & 2.27 & 2.01 & 2.06 & 2.47 & 2.34 & 2.39 & 2.60 & 2.47 & 2.51 & 3.97 & 3.66 & 3.71 \\
\hline 21 & 2.41 & 1.99 & 2.09 & 2.43 & 2.26 & 2.31 & 2.66 & 2.57 & 2.60 & 3.83 & 3.61 & 3.73 \\
\hline 22 & 2.18 & 2.00 & 2.06 & 2.30 & 2.22 & 2.25 & 2.78 & 2.60 & 2.64 & 3.61 & 3.37 & 3.49 \\
\hline 23 & 2.41 & 2.00 & 2.07 & 2.26 & 2.17 & 2.20 & 2.76 & 2.58 & 2.62 & 3.37 & 3.15 & 3.26 \\
\hline 24 & 2.58 & 2.11 & 2.18 & 2.27 & 2.16 & 2.19 & 2.74 & 2.50 & 2.56 & 3.15 & 2.96 & 3.05 \\
\hline 25 & 2.96 & 2.16 & 2.31 & 2.52 & 2.18 & 2.26 & 2.66 & 2.43 & 2.49 & 2.96 & 2.81 & 2.89 \\
\hline 26 & 3.24 & 2.33 & 2.52 & 2.61 & 2.26 & 2.38 & 2.94 & 2.39 & 2.53 & 2.82 & 2.67 & 2.74 \\
\hline 27 & 3.25 & 2.46 & 2.66 & 2.76 & 2.31 & 2.45 & 3.65 & 2.94 & 3.36 & 2.67 & 2.58 & 2.62 \\
\hline 28 & 3.24 & 2.48 & 2.66 & 2.91 & 2.33 & 2.51 & 3.74 & 3.65 & 3.71 & 2.81 & 2.54 & 2.59 \\
\hline 29 & 2.90 & 2.38 & 2.55 & 2.74 & 2.37 & 2.48 & 3.70 & 3.52 & 3.61 & 2.55 & 2.47 & 2.50 \\
\hline 30 & 2.67 & 2.27 & 2.40 & 2.67 & 2.33 & 2.49 & 3.52 & 3.31 & 3.42 & 2.52 & 2.44 & 2.46 \\
\hline 31 & - & --- & --- & 2.96 & 2.60 & 2.77 & . & --- & --- & 2.62 & 2.47 & 2.52 \\
\hline MONTH & 3.25 & 1.84 & 2.18 & 2.96 & 1.96 & 2.27 & 3.74 & 2.39 & 2.81 & 4.41 & 2.24 & 2.89 \\
\hline
\end{tabular}


Table A26. Stage data collected May-November 2001 at station 101 (USGS identifier 01022820) Northeast Creek at Route 3 bridge near Bar Harbor, ME [Data are presented as minimum, mean and maximum daily values of continuous data collected at 15-minute intervals.]

\begin{tabular}{|c|c|c|c|c|c|c|c|c|c|c|c|c|}
\hline DAY & MAX & MIN & MEAN & MAX & MIN & MEAN & MAX & MIN & MEAN & $\operatorname{MAX}$ & MIN & MEAN \\
\hline & \multicolumn{3}{|c|}{ JANUARY } & \multicolumn{3}{|c|}{ FEBRUARY } & \multicolumn{3}{|c|}{ MARCH } & \multicolumn{3}{|c|}{ APRIL } \\
\hline $\begin{array}{l}1 \\
2 \\
3 \\
4 \\
5\end{array}$ & $\begin{array}{l}2.64 \\
2.64 \\
2.59 \\
2.51 \\
2.45\end{array}$ & $\begin{array}{l}2.56 \\
2.59 \\
2.51 \\
2.44 \\
2.39\end{array}$ & $\begin{array}{l}2.61 \\
2.62 \\
2.55 \\
2.47 \\
2.42\end{array}$ & $\begin{array}{l}2.26 \\
2.23 \\
2.44 \\
2.35 \\
2.66\end{array}$ & $\begin{array}{l}2.21 \\
2.22 \\
2.22 \\
2.20 \\
2.20\end{array}$ & $\begin{array}{l}2.22 \\
2.22 \\
2.25 \\
2.23 \\
2.34\end{array}$ & $\begin{array}{r}2.43 \\
2.44 \\
--- \\
2.30 \\
2.60\end{array}$ & $\begin{array}{l}2.36 \\
2.30 \\
2.26 \\
2.21 \\
2.22\end{array}$ & $\begin{array}{r}2.40 \\
2.34 \\
--- \\
2.25 \\
2.29\end{array}$ & $\begin{array}{l}3.05 \\
3.05 \\
2.96 \\
2.89 \\
2.95\end{array}$ & $\begin{array}{l}3.00 \\
2.96 \\
2.87 \\
2.83 \\
2.82\end{array}$ & $\begin{array}{l}3.03 \\
3.00 \\
2.91 \\
2.85 \\
2.87\end{array}$ \\
\hline $\begin{array}{r}6 \\
7 \\
8 \\
9 \\
10\end{array}$ & $\begin{array}{l}2.89 \\
3.08 \\
3.45 \\
4.03 \\
3.94\end{array}$ & $\begin{array}{l}2.38 \\
2.42 \\
2.38 \\
2.46 \\
2.63\end{array}$ & $\begin{array}{l}2.51 \\
2.52 \\
2.62 \\
2.82 \\
2.89\end{array}$ & $\begin{array}{l}2.96 \\
3.43 \\
3.68 \\
4.25 \\
4.10\end{array}$ & $\begin{array}{l}2.25 \\
2.23 \\
2.25 \\
2.32 \\
2.59\end{array}$ & $\begin{array}{l}2.37 \\
2.44 \\
2.53 \\
2.74 \\
2.93\end{array}$ & $\begin{array}{l}3.61 \\
3.20 \\
3.58 \\
3.88 \\
4.04\end{array}$ & $\begin{array}{l}2.21 \\
2.33 \\
2.27 \\
2.22 \\
2.35\end{array}$ & $\begin{array}{l}2.56 \\
2.57 \\
2.56 \\
2.66 \\
2.71\end{array}$ & $\begin{array}{l}3.15 \\
3.28 \\
3.40 \\
3.45 \\
3.56\end{array}$ & $\begin{array}{l}2.92 \\
3.00 \\
3.06 \\
3.10 \\
3.22\end{array}$ & $\begin{array}{l}2.98 \\
3.07 \\
3.11 \\
3.17 \\
3.27\end{array}$ \\
\hline $\begin{array}{l}11 \\
12 \\
13 \\
14 \\
15\end{array}$ & $\begin{array}{l}4.20 \\
3.71 \\
3.47 \\
2.95 \\
2.62\end{array}$ & $\begin{array}{l}2.62 \\
2.60 \\
2.56 \\
2.50 \\
2.37\end{array}$ & $\begin{array}{l}2.93 \\
2.80 \\
2.74 \\
2.62 \\
2.46\end{array}$ & $\begin{array}{l}2.78 \\
2.90 \\
2.99 \\
2.71 \\
2.87\end{array}$ & $\begin{array}{l}2.66 \\
2.64 \\
2.61 \\
2.56 \\
2.58\end{array}$ & $\begin{array}{l}2.70 \\
2.69 \\
2.67 \\
2.59 \\
2.69\end{array}$ & $\begin{array}{l}3.32 \\
3.04 \\
3.12 \\
3.24 \\
2.45\end{array}$ & $\begin{array}{l}2.32 \\
2.19 \\
2.17 \\
2.27 \\
2.28\end{array}$ & $\begin{array}{l}2.53 \\
2.38 \\
2.39 \\
2.43 \\
2.30\end{array}$ & $\begin{array}{c}3.45 \\
--- \\
--- \\
--- \\
---\end{array}$ & $\begin{array}{c}3.28 \\
--- \\
--- \\
--- \\
---\end{array}$ & $\begin{array}{c}3.33 \\
--- \\
--- \\
--- \\
--\end{array}$ \\
\hline $\begin{array}{l}16 \\
17 \\
18 \\
19 \\
20\end{array}$ & $\begin{array}{r}2.74 \\
2.38 \\
--- \\
--- \\
---\end{array}$ & $\begin{array}{c}2.29 \\
2.23 \\
--- \\
--- \\
---\end{array}$ & $\begin{array}{r}2.39 \\
2.27 \\
--- \\
--- \\
---\end{array}$ & $\begin{array}{l}2.73 \\
2.82 \\
2.72 \\
2.68 \\
2.61\end{array}$ & $\begin{array}{l}2.71 \\
2.72 \\
2.68 \\
2.61 \\
2.58\end{array}$ & $\begin{array}{l}2.72 \\
2.74 \\
2.70 \\
2.65 \\
2.59\end{array}$ & $\begin{array}{l}2.35 \\
2.35 \\
2.34 \\
2.47 \\
2.64\end{array}$ & $\begin{array}{l}2.32 \\
2.32 \\
2.32 \\
2.34 \\
2.47\end{array}$ & $\begin{array}{l}2.33 \\
2.33 \\
2.33 \\
2.38 \\
2.53\end{array}$ & $\begin{array}{l}--- \\
--- \\
--- \\
--- \\
---\end{array}$ & $\begin{array}{l}--- \\
--- \\
--- \\
--- \\
--\end{array}$ & $\begin{array}{l}--- \\
--- \\
--- \\
---\end{array}$ \\
\hline $\begin{array}{l}21 \\
22 \\
23 \\
24 \\
25\end{array}$ & $\begin{array}{l}--- \\
--- \\
--- \\
--- \\
---\end{array}$ & $\begin{array}{l}--- \\
--- \\
--- \\
--- \\
---\end{array}$ & $\begin{array}{l}--- \\
--- \\
--- \\
--- \\
---\end{array}$ & $\begin{array}{l}2.79 \\
2.59 \\
2.90 \\
2.50 \\
2.64\end{array}$ & $\begin{array}{l}2.57 \\
2.52 \\
2.49 \\
2.43 \\
2.39\end{array}$ & $\begin{array}{l}2.60 \\
2.56 \\
2.55 \\
2.46 \\
2.42\end{array}$ & $\begin{array}{l}2.75 \\
3.15 \\
3.33 \\
3.46 \\
3.44\end{array}$ & $\begin{array}{l}2.64 \\
2.75 \\
3.01 \\
3.33 \\
3.37\end{array}$ & $\begin{array}{l}2.68 \\
2.84 \\
3.18 \\
3.39 \\
3.41\end{array}$ & $\begin{array}{c}--- \\
--- \\
--- \\
--- \\
3.33\end{array}$ & $\begin{array}{l}--- \\
--- \\
--- \\
--- \\
3.09\end{array}$ & $\begin{array}{l}--- \\
--- \\
--- \\
--16\end{array}$ \\
\hline $\begin{array}{l}26 \\
27 \\
28 \\
29 \\
30 \\
31\end{array}$ & $\begin{array}{l}2.44 \\
2.75 \\
2.29 \\
2.13 \\
2.15 \\
2.28\end{array}$ & $\begin{array}{l}2.14 \\
2.12 \\
2.13 \\
2.09 \\
2.08 \\
2.15\end{array}$ & $\begin{array}{l}2.20 \\
2.25 \\
2.17 \\
2.11 \\
2.10 \\
2.20\end{array}$ & $\begin{array}{r}2.74 \\
2.47 \\
2.47 \\
--- \\
--- \\
---\end{array}$ & $\begin{array}{l}2.42 \\
2.44 \\
2.42 \\
---- \\
---\end{array}$ & $\begin{array}{r}2.48 \\
2.45 \\
2.44 \\
--- \\
--- \\
---\end{array}$ & $\begin{array}{l}3.37 \\
3.25 \\
3.08 \\
2.93 \\
2.83 \\
3.08\end{array}$ & $\begin{array}{l}3.24 \\
3.08 \\
2.93 \\
2.83 \\
2.71 \\
2.75\end{array}$ & $\begin{array}{l}3.29 \\
3.14 \\
2.99 \\
2.87 \\
2.78 \\
2.90\end{array}$ & $\begin{array}{r}3.24 \\
3.10 \\
3.01 \\
2.72 \\
2.56 \\
---\end{array}$ & $\begin{array}{r}2.91 \\
2.75 \\
2.65 \\
2.53 \\
2.44 \\
---\end{array}$ & $\begin{array}{l}3.02 \\
2.87 \\
2.76 \\
2.60 \\
2.49 \\
---\end{array}$ \\
\hline MONTH & 4.20 & 2.08 & 2.49 & 4.25 & 2.20 & 2.53 & 7.20 & 2.17 & 2.66 & 3.56 & 2.44 & 2.97 \\
\hline \multirow[t]{2}{*}{ DAY } & MAX & MIN & MEAN & MAX & MIN & MEAN & MAX & MIN & MEAN & $\operatorname{MAX}$ & MIN & MEAN \\
\hline & \multicolumn{3}{|c|}{ MAY } & \multicolumn{3}{|c|}{ JUNE } & \multicolumn{3}{|c|}{ JULY } & \multicolumn{3}{|c|}{ AUGUST } \\
\hline $\begin{array}{l}1 \\
2 \\
3 \\
4 \\
5\end{array}$ & $\begin{array}{l}2.69 \\
2.69 \\
2.54 \\
2.67 \\
2.86\end{array}$ & $\begin{array}{l}2.42 \\
2.50 \\
2.38 \\
2.36 \\
2.41\end{array}$ & $\begin{array}{l}2.55 \\
2.59 \\
2.46 \\
2.43 \\
2.53\end{array}$ & $\begin{array}{l}2.55 \\
2.89 \\
3.16 \\
3.15 \\
3.08\end{array}$ & $\begin{array}{l}2.30 \\
2.34 \\
2.67 \\
2.80 \\
2.73\end{array}$ & $\begin{array}{l}2.39 \\
2.44 \\
2.76 \\
2.88 \\
2.84\end{array}$ & $\begin{array}{l}2.78 \\
2.52 \\
2.48 \\
2.59 \\
2.65\end{array}$ & $\begin{array}{l}2.12 \\
2.14 \\
2.12 \\
2.12 \\
2.14\end{array}$ & $\begin{array}{l}2.25 \\
2.26 \\
2.22 \\
2.21 \\
2.23\end{array}$ & $\begin{array}{l}2.26 \\
2.22 \\
2.30 \\
2.30 \\
2.25\end{array}$ & $\begin{array}{l}2.00 \\
1.99 \\
1.99 \\
2.00 \\
1.98\end{array}$ & $\begin{array}{l}2.06 \\
2.05 \\
2.04 \\
2.06 \\
2.05\end{array}$ \\
\hline $\begin{array}{r}6 \\
7 \\
8 \\
9 \\
10\end{array}$ & $\begin{array}{l}2.76 \\
2.69 \\
2.70 \\
2.65 \\
2.61\end{array}$ & $\begin{array}{l}2.40 \\
2.34 \\
2.31 \\
2.27 \\
2.26\end{array}$ & $\begin{array}{l}2.54 \\
2.47 \\
2.44 \\
2.40 \\
2.38\end{array}$ & $\begin{array}{l}3.08 \\
2.93 \\
2.71 \\
2.56 \\
2.39\end{array}$ & $\begin{array}{l}2.63 \\
2.47 \\
2.34 \\
2.27 \\
2.22\end{array}$ & $\begin{array}{l}2.75 \\
2.62 \\
2.47 \\
2.37 \\
2.28\end{array}$ & $\begin{array}{l}2.67 \\
2.45 \\
2.34 \\
2.32 \\
2.21\end{array}$ & $\begin{array}{l}2.16 \\
2.14 \\
2.14 \\
2.14 \\
2.12\end{array}$ & $\begin{array}{l}2.27 \\
2.21 \\
2.19 \\
2.20 \\
2.15\end{array}$ & $\begin{array}{l}2.10 \\
2.06 \\
1.99 \\
1.95 \\
1.91\end{array}$ & $\begin{array}{l}1.94 \\
1.93 \\
1.88 \\
1.89 \\
1.86\end{array}$ & $\begin{array}{l}1.99 \\
1.96 \\
1.92 \\
1.92 \\
1.89\end{array}$ \\
\hline $\begin{array}{l}11 \\
12 \\
13 \\
14 \\
15\end{array}$ & $\begin{array}{l}2.50 \\
2.34 \\
2.21 \\
2.13 \\
2.11\end{array}$ & $\begin{array}{l}2.23 \\
2.16 \\
2.13 \\
2.08 \\
2.08\end{array}$ & $\begin{array}{l}2.32 \\
2.25 \\
2.16 \\
2.10 \\
2.09\end{array}$ & $\begin{array}{l}2.23 \\
2.22 \\
2.23 \\
2.22 \\
2.18\end{array}$ & $\begin{array}{l}2.16 \\
2.16 \\
2.21 \\
2.18 \\
2.13\end{array}$ & $\begin{array}{l}2.19 \\
2.20 \\
2.22 \\
2.20 \\
2.15\end{array}$ & $\begin{array}{l}2.16 \\
2.10 \\
2.07 \\
2.05 \\
2.05\end{array}$ & $\begin{array}{l}2.10 \\
2.06 \\
2.05 \\
2.05 \\
2.05\end{array}$ & $\begin{array}{l}2.12 \\
2.08 \\
2.06 \\
2.05 \\
2.05\end{array}$ & $\begin{array}{l}1.89 \\
1.84 \\
1.81 \\
1.87 \\
2.10\end{array}$ & $\begin{array}{l}1.84 \\
1.80 \\
1.78 \\
1.78 \\
1.78\end{array}$ & $\begin{array}{l}1.86 \\
1.82 \\
1.79 \\
1.79 \\
1.82\end{array}$ \\
\hline $\begin{array}{l}16 \\
17 \\
18 \\
19 \\
20\end{array}$ & $\begin{array}{l}2.21 \\
2.21 \\
2.20 \\
2.42 \\
2.48\end{array}$ & $\begin{array}{l}2.08 \\
2.19 \\
2.14 \\
2.15 \\
2.42\end{array}$ & $\begin{array}{l}2.13 \\
2.20 \\
2.17 \\
2.26 \\
2.44\end{array}$ & $\begin{array}{l}2.14 \\
2.10 \\
2.52 \\
2.63 \\
2.70\end{array}$ & $\begin{array}{l}2.08 \\
2.08 \\
2.10 \\
2.36 \\
2.31\end{array}$ & $\begin{array}{l}2.11 \\
2.08 \\
2.35 \\
2.44 \\
2.41\end{array}$ & $\begin{array}{l}2.08 \\
2.25 \\
2.56 \\
2.97 \\
3.34\end{array}$ & $\begin{array}{l}2.05 \\
2.05 \\
2.05 \\
2.10 \\
2.21\end{array}$ & $\begin{array}{l}2.05 \\
2.07 \\
2.11 \\
2.24 \\
2.40\end{array}$ & $\begin{array}{l}2.56 \\
3.12 \\
3.47 \\
3.62 \\
3.58\end{array}$ & $\begin{array}{l}1.83 \\
2.04 \\
2.24 \\
2.38 \\
2.52\end{array}$ & $\begin{array}{l}1.95 \\
2.25 \\
2.50 \\
2.66 \\
2.77\end{array}$ \\
\hline $\begin{array}{l}21 \\
22 \\
23 \\
24 \\
25\end{array}$ & $\begin{array}{l}2.49 \\
2.68 \\
2.75 \\
2.80 \\
2.88\end{array}$ & $\begin{array}{l}2.39 \\
2.35 \\
2.35 \\
2.35 \\
2.33\end{array}$ & $\begin{array}{l}2.43 \\
2.41 \\
2.46 \\
2.50 \\
2.50\end{array}$ & $\begin{array}{l}2.94 \\
2.94 \\
3.16 \\
3.28 \\
3.14\end{array}$ & $\begin{array}{l}2.26 \\
2.28 \\
2.39 \\
2.43 \\
2.42\end{array}$ & $\begin{array}{l}2.40 \\
2.45 \\
2.57 \\
2.65 \\
2.61\end{array}$ & $\begin{array}{l}3.45 \\
3.59 \\
3.59 \\
3.46 \\
3.14\end{array}$ & $\begin{array}{l}2.34 \\
2.44 \\
2.48 \\
2.48 \\
2.47\end{array}$ & $\begin{array}{l}2.55 \\
2.69 \\
2.75 \\
2.75 \\
2.64\end{array}$ & $\begin{array}{l}3.38 \\
3.18 \\
2.98 \\
2.70 \\
2.42\end{array}$ & $\begin{array}{l}2.57 \\
2.54 \\
2.43 \\
2.28 \\
2.17\end{array}$ & $\begin{array}{l}2.80 \\
2.74 \\
2.60 \\
2.41 \\
2.24\end{array}$ \\
\hline $\begin{array}{l}26 \\
27 \\
28 \\
29 \\
30 \\
31\end{array}$ & $\begin{array}{l}2.78 \\
2.80 \\
2.74 \\
2.84 \\
2.75 \\
2.53\end{array}$ & $\begin{array}{l}2.31 \\
2.30 \\
2.33 \\
2.43 \\
2.50 \\
2.36\end{array}$ & $\begin{array}{l}2.45 \\
2.47 \\
2.48 \\
2.60 \\
2.56 \\
2.45\end{array}$ & $\begin{array}{l}2.87 \\
2.64 \\
2.48 \\
2.41 \\
2.44\end{array}$ & $\begin{array}{l}2.35 \\
2.29 \\
2.18 \\
2.16 \\
2.12\end{array}$ & $\begin{array}{l}2.50 \\
2.38 \\
2.29 \\
2.24 \\
2.21\end{array}$ & $\begin{array}{l}2.87 \\
2.46 \\
2.27 \\
2.18 \\
2.18 \\
2.23\end{array}$ & $\begin{array}{l}2.39 \\
2.21 \\
2.12 \\
2.06 \\
2.02 \\
2.02\end{array}$ & $\begin{array}{l}2.52 \\
2.31 \\
2.18 \\
2.10 \\
2.07 \\
2.06\end{array}$ & $\begin{array}{l}2.23 \\
2.20 \\
2.12 \\
2.09 \\
2.11 \\
2.18\end{array}$ & $\begin{array}{l}2.08 \\
2.04 \\
2.02 \\
1.99 \\
1.97 \\
1.96\end{array}$ & $\begin{array}{l}2.13 \\
2.08 \\
2.05 \\
2.03 \\
2.01 \\
2.01\end{array}$ \\
\hline MONTH & 2.88 & 2.08 & 2.39 & 3.28 & 2.08 & 2.42 & 3.59 & 2.02 & 2.26 & 3.62 & 1.78 & 2.14 \\
\hline
\end{tabular}


Table A26. Stage data collected May-November 2001 at station 101 (USGS identifier 01022820) Northeast Creek at Route 3 bridge near Bar Harbor, ME - .Continued.

GAGE HEIGHT, IN FEET

\begin{tabular}{|c|c|c|c|c|c|c|c|c|c|}
\hline \multirow[t]{2}{*}{ DAY } & MAX & MIN & MEAN & MAX & MIN & MEAN & MAX & MIN & MEAN \\
\hline & \multicolumn{3}{|c|}{ SEPTEMBER } & \multicolumn{3}{|c|}{ OCTOBER } & \multicolumn{3}{|c|}{ NOVEMBER } \\
\hline $\begin{array}{l}1 \\
2 \\
3 \\
4 \\
5\end{array}$ & $\begin{array}{l}2.16 \\
2.14 \\
2.15 \\
2.09 \\
2.04\end{array}$ & $\begin{array}{l}2.01 \\
2.01 \\
1.99 \\
1.99 \\
1.97\end{array}$ & $\begin{array}{l}2.07 \\
2.05 \\
2.03 \\
2.02 \\
1.98\end{array}$ & $\begin{array}{l}2.19 \\
2.14 \\
2.16 \\
2.25 \\
2.22\end{array}$ & $\begin{array}{l}1.96 \\
2.05 \\
2.06 \\
2.07 \\
2.08\end{array}$ & $\begin{array}{l}2.01 \\
2.09 \\
2.10 \\
2.13 \\
2.12\end{array}$ & $\begin{array}{l}2.05 \\
2.18 \\
2.30 \\
2.35 \\
2.56\end{array}$ & $\begin{array}{l}1.99 \\
2.02 \\
2.06 \\
2.08 \\
2.12\end{array}$ & $\begin{array}{l}2.03 \\
2.09 \\
2.15 \\
2.18 \\
2.25\end{array}$ \\
\hline $\begin{array}{r}6 \\
7 \\
8 \\
9 \\
10\end{array}$ & $\begin{array}{l}1.97 \\
1.96 \\
1.91 \\
1.88 \\
1.86\end{array}$ & $\begin{array}{l}1.93 \\
1.90 \\
1.88 \\
1.86 \\
1.83\end{array}$ & $\begin{array}{l}1.95 \\
1.92 \\
1.89 \\
1.87 \\
1.84\end{array}$ & $\begin{array}{l}2.45 \\
2.19 \\
2.10 \\
2.02 \\
1.97\end{array}$ & $\begin{array}{l}2.06 \\
2.09 \\
2.01 \\
1.96 \\
1.94\end{array}$ & $\begin{array}{l}2.20 \\
2.13 \\
2.04 \\
1.98 \\
1.95\end{array}$ & $\begin{array}{c}2.32 \\
--- \\
--- \\
--- \\
---\end{array}$ & $\begin{array}{c}2.14 \\
--- \\
--- \\
--- \\
---\end{array}$ & $\begin{array}{c}2.21 \\
--- \\
--- \\
--- \\
---\end{array}$ \\
\hline $\begin{array}{l}11 \\
12 \\
13 \\
14 \\
15\end{array}$ & $\begin{array}{l}1.84 \\
1.91 \\
2.21 \\
2.51 \\
2.87\end{array}$ & $\begin{array}{l}1.80 \\
1.79 \\
1.81 \\
1.93 \\
2.10\end{array}$ & $\begin{array}{l}1.82 \\
1.83 \\
1.91 \\
2.06 \\
2.27\end{array}$ & $\begin{array}{l}1.99 \\
2.11 \\
2.64 \\
2.97 \\
3.03\end{array}$ & $\begin{array}{l}1.92 \\
1.94 \\
2.02 \\
2.23 \\
2.44\end{array}$ & $\begin{array}{l}1.95 \\
1.99 \\
2.16 \\
2.45 \\
2.64\end{array}$ & $\begin{array}{l}--- \\
--- \\
--- \\
--- \\
---\end{array}$ & $\begin{array}{l}--- \\
--- \\
--- \\
--- \\
---\end{array}$ & $\begin{array}{l}--- \\
--- \\
--- \\
--- \\
---\end{array}$ \\
\hline $\begin{array}{l}16 \\
17 \\
18 \\
19 \\
20\end{array}$ & $\begin{array}{l}3.22 \\
3.38 \\
3.42 \\
3.35 \\
3.19\end{array}$ & $\begin{array}{l}2.30 \\
2.44 \\
2.56 \\
2.63 \\
2.57\end{array}$ & $\begin{array}{l}2.51 \\
2.67 \\
2.81 \\
2.81 \\
2.72\end{array}$ & $\begin{array}{l}3.26 \\
3.66 \\
2.97 \\
2.88 \\
2.79\end{array}$ & $\begin{array}{l}2.47 \\
2.67 \\
2.55 \\
2.39 \\
2.32\end{array}$ & $\begin{array}{l}2.71 \\
2.90 \\
2.69 \\
2.53 \\
2.45\end{array}$ & $\begin{array}{l}--- \\
--- \\
--- \\
--- \\
---\end{array}$ & $\begin{array}{l}--- \\
--- \\
--- \\
--- \\
---\end{array}$ & $\begin{array}{l}--- \\
--- \\
--- \\
--- \\
---\end{array}$ \\
\hline $\begin{array}{l}21 \\
22 \\
23 \\
24 \\
25\end{array}$ & $\begin{array}{l}2.93 \\
2.63 \\
2.32 \\
2.16 \\
2.14\end{array}$ & $\begin{array}{l}2.47 \\
2.32 \\
2.16 \\
2.07 \\
2.05\end{array}$ & $\begin{array}{l}2.59 \\
2.44 \\
2.23 \\
2.11 \\
2.08\end{array}$ & $\begin{array}{l}2.38 \\
2.21 \\
2.10 \\
2.04 \\
2.03\end{array}$ & $\begin{array}{l}2.21 \\
2.10 \\
2.04 \\
2.02 \\
2.00\end{array}$ & $\begin{array}{l}2.28 \\
2.14 \\
2.08 \\
2.03 \\
2.02\end{array}$ & $\begin{array}{l}--- \\
--- \\
--- \\
--- \\
---\end{array}$ & $\begin{array}{l}--- \\
--- \\
--- \\
--- \\
---\end{array}$ & $\begin{array}{l}--- \\
--- \\
--- \\
--- \\
---\end{array}$ \\
\hline $\begin{array}{l}26 \\
27 \\
28 \\
29 \\
30 \\
31\end{array}$ & $\begin{array}{r}2.14 \\
2.09 \\
2.05 \\
2.02 \\
---- \\
---\end{array}$ & $\begin{array}{r}2.09 \\
2.04 \\
2.00 \\
1.98 \\
--- \\
---\end{array}$ & $\begin{array}{r}2.11 \\
2.06 \\
2.02 \\
1.99 \\
---- \\
---\end{array}$ & $\begin{array}{l}2.02 \\
1.99 \\
1.97 \\
1.95 \\
1.94 \\
2.01\end{array}$ & $\begin{array}{l}1.98 \\
1.95 \\
1.94 \\
1.92 \\
1.89 \\
1.91\end{array}$ & $\begin{array}{l}2.00 \\
1.97 \\
1.95 \\
1.94 \\
1.92 \\
1.93\end{array}$ & $\begin{array}{l}--- \\
--- \\
--- \\
--- \\
--- \\
---\end{array}$ & $\begin{array}{l}--- \\
--- \\
--- \\
--- \\
--- \\
---\end{array}$ & $\begin{array}{l}--- \\
--- \\
--- \\
--- \\
--- \\
---\end{array}$ \\
\hline MONTH & 3.42 & 1.79 & 2.16 & 3.66 & 1.89 & 2.18 & 2.56 & 1.99 & 2.15 \\
\hline
\end{tabular}


Table A27. Stage data collected September -November 2000 at station 103 (USGS identifier 442507068185301) Northeast Creek near Bar Harbor, ME [Data are presented as minimum, mean and maximum daily values of continuous data collected at 15-minute intervals.]

\begin{tabular}{|c|c|c|c|c|c|c|c|c|c|}
\hline & & & & & AGE & IGHT & $\mathrm{N} F E \mathrm{E}$ & & \\
\hline DAY & MAX & MIN & MEAN & MAX & MIN & MEAN & MAX & MIN & MEAN \\
\hline & & PTEME & & & CTOBER & & & VEMBER & \\
\hline 1 & --- & --- & --- & --- & --- & --- & 1.68 & 1.56 & 1.64 \\
\hline 2 & --- & --- & --- & --- & --- & --- & 1.71 & 1.60 & 1.66 \\
\hline 3 & --- & --- & --- & --- & --- & --- & 1.60 & 1.47 & 1.55 \\
\hline 4 & --- & --- & --- & --- & --- & --- & 1.47 & 1.35 & 1.40 \\
\hline 5 & --- & --- & --- & --- & --- & --- & 1.35 & 1.26 & 1.30 \\
\hline 6 & --- & --- & --- & --- & --- & --- & 1.26 & 1.19 & 1.23 \\
\hline 7 & --- & --- & --- & --- & --- & --- & 1.24 & 1.15 & 1.19 \\
\hline 8 & --- & --- & --- & --- & --- & --- & 1.17 & 1.10 & 1.13 \\
\hline 9 & --- & --- & --- & --- & --- & --- & 1.26 & 1.04 & 1.15 \\
\hline 10 & --- & --- & --- & --- & --- & --- & 1.47 & 1.23 & 1.32 \\
\hline 11 & --- & --- & --- & --- & --- & --- & 1.61 & 1.26 & 1.38 \\
\hline 12 & --- & --- & --- & 0.93 & 0.76 & 0.82 & 1.62 & 1.24 & 1.37 \\
\hline 13 & --- & --- & --- & 0.97 & 0.74 & 0.82 & 1.59 & 1.19 & 1.33 \\
\hline 14 & --- & --- & --- & 1.09 & 0.72 & 0.85 & 1.64 & 1.17 & 1.36 \\
\hline 15 & --- & --- & --- & 0.96 & 0.78 & 0.86 & 1.73 & 1.38 & 1.50 \\
\hline 16 & --- & --- & --- & 0.96 & 0.73 & 0.82 & 1.53 & 1.40 & 1.44 \\
\hline 17 & --- & --- & --- & 0.95 & 0.72 & 0.81 & 1.51 & 1.33 & 1.39 \\
\hline 18 & --- & --- & --- & 1.01 & 0.69 & 0.79 & 1.33 & 1.18 & 1.24 \\
\hline 19 & --- & --- & --- & 1.17 & 0.86 & 0.98 & 1.18 & 1.06 & 1.11 \\
\hline 20 & --- & --- & --- & 1.03 & 0.90 & 0.97 & 1.18 & 1.03 & 1.09 \\
\hline 21 & --- & --- & --- & 1.08 & 0.85 & 0.92 & 1.25 & 1.16 & 1.20 \\
\hline 22 & --- & --- & --- & 0.89 & 0.78 & 0.84 & 1.31 & 1.17 & 1.22 \\
\hline 23 & --- & --- & --- & 0.92 & 0.74 & 0.79 & 1.30 & 1.14 & 1.20 \\
\hline 24 & --- & --- & --- & 1.02 & 0.73 & 0.81 & 1.25 & 1.07 & 1.13 \\
\hline 25 & --- & --- & --- & 1.06 & 0.73 & 0.86 & 1.18 & 1.02 & 1.06 \\
\hline 26 & --- & --- & --- & 1.14 & 0.85 & 1.00 & 1.58 & 1.00 & 1.15 \\
\hline 27 & --- & --- & --- & 1.22 & 0.89 & 1.04 & 2.32 & 1.58 & 2.03 \\
\hline 28 & --- & --- & --- & 1.33 & 0.95 & 1.10 & & --- & --- \\
\hline 29 & --- & --- & --- & 1.24 & 0.95 & 1.06 & --- & --- & --- \\
\hline 30 & --- & --- & --- & 1.20 & 0.91 & 1.06 & --- & --- & --- \\
\hline 31 & & --- & & 1.56 & 1.17 & 1.34 & --- & --- & --- \\
\hline MONTH & --- & --- & --- & 1.56 & 0.69 & 0.93 & 2.32 & 1.00 & 1.32 \\
\hline
\end{tabular}


Table A28. Stage data collected May -November 2001 at station 103 (USGS identifier 442507068185301) Northeast Creek near Bar Harbor, ME [Data are presented as minimum, mean and maximum daily values of continuous data collected at 15-minute intervals.]

GAGE HEIGHT, IN FEET

\begin{tabular}{|c|c|c|c|c|c|c|c|c|c|c|c|c|}
\hline DAY & MAX & MIN & MEAN & MAX & MIN & MEAN & MAX & MIN & MEAN & MAX & MIN & MEAN \\
\hline & & MAY & & & JUNE & & \multicolumn{3}{|c|}{ JULY } & \multicolumn{3}{|c|}{ AUGUST } \\
\hline 1 & --- & --- & --- & --- & --- & --- & 2.67 & 2.26 & 2.39 & 2.40 & 2.28 & 2.34 \\
\hline 2 & --- & --- & --- & --- & --- & --- & 2.54 & 2.30 & 2.40 & 2.38 & 2.27 & 2.33 \\
\hline 3 & --- & --- & --- & --- & --- & --- & 2.50 & 2.27 & 2.37 & 2.44 & 2.27 & 2.34 \\
\hline 4 & --- & --- & --- & --- & --- & --- & 2.55 & 2.27 & 2.39 & 2.41 & 2.26 & 2.33 \\
\hline 5 & --- & --- & --- & --- & --- & --- & 2.63 & 2.32 & 2.43 & 2.38 & 2.25 & 2.31 \\
\hline 6 & --- & --- & --- & --- & --- & --- & 2.55 & 2.33 & 2.44 & 2.34 & 2.22 & 2.28 \\
\hline 7 & --- & --- & --- & --- & --- & --- & 2.45 & 2.31 & 2.39 & 2.30 & 2.19 & 2.25 \\
\hline 8 & --- & --- & --- & --- & --- & --- & 2.46 & 2.31 & 2.39 & 2.26 & 2.15 & 2.19 \\
\hline 9 & --- & --- & --- & --- & --- & --- & 2.43 & 2.32 & 2.37 & 2.28 & 2.16 & 2.19 \\
\hline 10 & --- & --- & --- & --- & --- & --- & 2.40 & 2.29 & 2.34 & 2.17 & 2.14 & 2.15 \\
\hline 11 & --- & --- & --- & --- & --- & --- & 2.41 & 2.28 & 2.34 & 2.19 & 2.09 & 2.13 \\
\hline 12 & --- & --- & --- & --- & --- & --- & 2.38 & 2.24 & 2.29 & 2.09 & 2.06 & \\
\hline 13 & --- & --- & --- & --- & --- & --- & 2.32 & 2.22 & 2.25 & 2.13 & 2.03 & 2.06 \\
\hline 14 & --- & --- & --- & --- & --- & --- & 2.35 & 2.20 & 2.23 & 2.08 & 2.03 & 2.04 \\
\hline 15 & --- & --- & --- & --- & --- & --- & 2.28 & 2.19 & 2.22 & 2.24 & 2.03 & 2.09 \\
\hline 16 & --- & --- & --- & --- & --- & --- & 2.29 & 2.19 & 2.21 & 2.59 & 2.10 & 2.23 \\
\hline & --- & --- & --- & --- & --- & --- & 2.36 & 2.20 & 2.24 & 3.03 & 2.32 & \\
\hline 18 & --- & --- & --- & --- & --- & --- & 2.58 & 2.26 & 2.35 & 3.27 & 2.56 & 2.76 \\
\hline 19 & --- & --- & --- & --- & --- & --- & 2.89 & 2.35 & 2.51 & 3.39 & 2.70 & 2.92 \\
\hline 20 & --- & --- & --- & --- & --- & --- & 3.13 & 2.51 & 2.67 & 3.40 & 2.81 & 3.01 \\
\hline 21 & --- & --- & --- & --- & --- & --- & 3.30 & 2.65 & 2.83 & 3.33 & 2.88 & 3.05 \\
\hline 22 & --- & --- & --- & 2.87 & 2.28 & 2.45 & 3.34 & 2.75 & 2.94 & 3.24 & 2.85 & 3.02 \\
\hline 23 & --- & --- & --- & 2.99 & 2.48 & 2.61 & 3.28 & 2.80 & 2.99 & 3.13 & 2.75 & 2.91 \\
\hline 24 & --- & --- & --- & 2.95 & 2.55 & 2.68 & 3.17 & 2.80 & 2.97 & 2.92 & 2.57 & 2.72 \\
\hline 25 & --- & --- & --- & 2.87 & 2.54 & 2.68 & 3.13 & 2.70 & 2.88 & 2.65 & 2.45 & 2.53 \\
\hline 26 & --- & --- & --- & 2.82 & 2.43 & 2.60 & 2.93 & 2.59 & 2.73 & 2.48 & 2.38 & \\
\hline 27 & --- & --- & --- & 2.65 & 2.35 & 2.50 & 2.63 & 2.46 & 2.54 & 2.46 & 2.34 & 2.37 \\
\hline 28 & --- & --- & --- & 2.52 & 2.34 & 2.41 & 2.47 & 2.37 & 2.43 & 2.45 & 2.34 & 2.39 \\
\hline 29 & --- & --- & --- & 2.46 & 2.30 & 2.35 & 2.39 & 2.31 & 2.35 & 2.41 & 2.35 & 2.38 \\
\hline 30 & --- & --- & --- & 2.47 & 2.29 & 2.37 & 2.37 & 2.30 & 2.33 & 2.40 & 2.32 & 2.35 \\
\hline 31 & --- & --- & - & --- & --- & --- & 2.39 & 2.29 & 2.33 & 2.45 & 2.30 & 2.35 \\
\hline MONTH & --- & --- & --- & 2.99 & 2.28 & 2.52 & 3.34 & 2.19 & 2.47 & 3.40 & 2.03 & 2.42 \\
\hline
\end{tabular}

\begin{tabular}{|c|c|c|c|c|c|c|c|c|c|}
\hline \multirow[t]{2}{*}{ DAY } & MAX & MIN & MEAN & MAX & MIN & MEAN & MAX & MIN & MEAN \\
\hline & \multicolumn{3}{|c|}{ SEPTEMBER } & \multicolumn{3}{|c|}{ OCTOBER } & \multicolumn{3}{|c|}{ NOVEMBER } \\
\hline $\begin{array}{l}1 \\
2 \\
3 \\
4 \\
5\end{array}$ & $\begin{array}{l}2.49 \\
2.44 \\
2.44 \\
2.41 \\
2.38\end{array}$ & $\begin{array}{l}2.36 \\
2.36 \\
2.33 \\
2.34 \\
2.30\end{array}$ & $\begin{array}{l}2.42 \\
2.40 \\
2.37 \\
2.37 \\
2.34\end{array}$ & $\begin{array}{l}2.54 \\
2.54 \\
2.56 \\
2.63 \\
2.60\end{array}$ & $\begin{array}{l}2.34 \\
2.45 \\
2.47 \\
2.49 \\
2.48\end{array}$ & $\begin{array}{l}2.40 \\
2.50 \\
2.50 \\
2.54 \\
2.53\end{array}$ & $\begin{array}{l}2.50 \\
2.63 \\
2.75 \\
2.80 \\
2.95\end{array}$ & $\begin{array}{l}2.44 \\
2.50 \\
2.55 \\
2.59 \\
2.60\end{array}$ & $\begin{array}{l}2.48 \\
2.56 \\
2.64 \\
2.68 \\
2.74\end{array}$ \\
\hline $\begin{array}{r}6 \\
7 \\
8 \\
9 \\
10\end{array}$ & $\begin{array}{l}2.32 \\
2.33 \\
2.28 \\
2.27 \\
2.23\end{array}$ & $\begin{array}{l}2.27 \\
2.25 \\
2.22 \\
2.20 \\
2.19\end{array}$ & $\begin{array}{l}2.29 \\
2.27 \\
2.25 \\
2.22 \\
2.21\end{array}$ & $\begin{array}{l}2.78 \\
2.60 \\
2.49 \\
2.41 \\
2.36\end{array}$ & $\begin{array}{l}2.47 \\
2.49 \\
2.41 \\
2.36 \\
2.33\end{array}$ & $\begin{array}{l}2.60 \\
2.54 \\
2.45 \\
2.38 \\
2.35\end{array}$ & $\begin{array}{c}2.82 \\
--- \\
--- \\
--- \\
---\end{array}$ & $\begin{array}{c}2.67 \\
--- \\
--- \\
--- \\
---\end{array}$ & $\begin{array}{c}2.72 \\
--- \\
--- \\
---\end{array}$ \\
\hline $\begin{array}{l}11 \\
12 \\
13 \\
14 \\
15\end{array}$ & $\begin{array}{l}2.22 \\
2.25 \\
2.47 \\
2.72 \\
3.02\end{array}$ & $\begin{array}{l}2.16 \\
2.16 \\
2.19 \\
2.31 \\
2.48\end{array}$ & $\begin{array}{l}2.20 \\
2.20 \\
2.26 \\
2.42 \\
2.62\end{array}$ & $\begin{array}{l}2.39 \\
2.49 \\
2.87 \\
3.17 \\
3.25\end{array}$ & $\begin{array}{l}2.32 \\
2.35 \\
2.42 \\
2.63 \\
2.84\end{array}$ & $\begin{array}{l}2.35 \\
2.39 \\
2.54 \\
2.83 \\
3.02\end{array}$ & $\begin{array}{l}--- \\
--- \\
--- \\
--- \\
---\end{array}$ & $\begin{array}{l}--- \\
--- \\
--- \\
--- \\
---\end{array}$ & $\begin{array}{l}--- \\
--- \\
--- \\
--- \\
--\end{array}$ \\
\hline $\begin{array}{l}16 \\
17 \\
18 \\
19 \\
20\end{array}$ & $\begin{array}{l}3.27 \\
3.40 \\
3.46 \\
3.44 \\
3.34\end{array}$ & $\begin{array}{l}2.67 \\
2.83 \\
2.96 \\
3.03 \\
2.95\end{array}$ & $\begin{array}{l}2.85 \\
3.01 \\
3.15 \\
3.17 \\
3.08\end{array}$ & $\begin{array}{l}3.40 \\
3.61 \\
3.26 \\
3.13 \\
3.07\end{array}$ & $\begin{array}{l}2.88 \\
3.06 \\
2.92 \\
2.77 \\
2.71\end{array}$ & $\begin{array}{l}3.07 \\
3.26 \\
3.08 \\
2.90 \\
2.83\end{array}$ & $\begin{array}{l}--- \\
--- \\
--- \\
--- \\
---\end{array}$ & $\begin{array}{l}--- \\
--- \\
--- \\
--- \\
---\end{array}$ & $\begin{array}{l}--- \\
--- \\
--- \\
--- \\
---\end{array}$ \\
\hline $\begin{array}{l}21 \\
22 \\
23 \\
24 \\
25\end{array}$ & $\begin{array}{l}3.17 \\
2.98 \\
2.73 \\
2.55 \\
2.53\end{array}$ & $\begin{array}{l}2.85 \\
2.73 \\
2.55 \\
2.46 \\
2.44\end{array}$ & $\begin{array}{l}2.97 \\
2.84 \\
2.64 \\
2.51 \\
2.46\end{array}$ & $\begin{array}{l}2.77 \\
2.62 \\
2.50 \\
2.46 \\
2.47\end{array}$ & $\begin{array}{l}2.62 \\
2.50 \\
2.44 \\
2.44 \\
2.43\end{array}$ & $\begin{array}{l}2.68 \\
2.56 \\
2.46 \\
2.45 \\
2.45\end{array}$ & $\begin{array}{l}--- \\
--- \\
--- \\
--- \\
---\end{array}$ & $\begin{array}{l}--- \\
--- \\
--- \\
--- \\
---\end{array}$ & $\begin{array}{l}--- \\
--- \\
--- \\
--- \\
---\end{array}$ \\
\hline $\begin{array}{l}26 \\
27 \\
28 \\
29 \\
30 \\
31\end{array}$ & $\begin{array}{r}2.54 \\
2.48 \\
2.42 \\
2.40 \\
2.37 \\
---\end{array}$ & $\begin{array}{r}2.48 \\
2.41 \\
2.39 \\
2.36 \\
2.35 \\
---\end{array}$ & $\begin{array}{l}2.52 \\
2.45 \\
2.41 \\
2.38 \\
2.36 \\
---\end{array}$ & $\begin{array}{l}2.45 \\
2.42 \\
2.40 \\
2.38 \\
2.39 \\
2.44\end{array}$ & $\begin{array}{l}2.42 \\
2.40 \\
2.36 \\
2.35 \\
2.35 \\
2.35\end{array}$ & $\begin{array}{l}2.44 \\
2.41 \\
2.39 \\
2.37 \\
2.37 \\
2.37\end{array}$ & $\begin{array}{l}--- \\
--- \\
--- \\
--- \\
--- \\
--\end{array}$ & $\begin{array}{l}--- \\
--- \\
--- \\
--- \\
--- \\
---\end{array}$ & $\begin{array}{l}--- \\
--- \\
--- \\
--- \\
--- \\
--\end{array}$ \\
\hline MONTH & 3.46 & 2.16 & 2.52 & 3.61 & 2.32 & 2.58 & 2.95 & 2.44 & 2.64 \\
\hline
\end{tabular}




\section{Appendix 4}

\section{Stage-discharge rating for station 101}


Table A29. Stage-discharge relation for station 101 (USGS identifier (01022820) Northeast Creek at Rt 3 bridge near Bar Harbor, $\mathrm{ME}$

[The stage-discharge rating for station 101 is only applicable for positive flow (outflow). Although the stage-discharge ratings can change over time, the rating for this site was stable during the study period. The reference mark used to determine this rating is a chiseled square on top of the upstream right wing wall (facing upstream) on the route 3 bridge. The orifice line for the gage was 60 feet upstream and southeast of bridge reference mark. An assumed gage datum of 12.00 feet was used to develop this rating, therefore the gage height values shown here do not correspond to actual NGVD 29 altitudes (For example, a pool stage of $2.00 \mathrm{ft}$ is 10 $\mathrm{ft}$ below the reference mark).]

\begin{tabular}{cccc}
\hline $\begin{array}{c}\text { Gage Height, } \\
\text { feet }\end{array}$ & $\begin{array}{c}\text { Discharge, } \\
\text { cubic feet per } \\
\text { second }\end{array}$ & $\begin{array}{c}\text { Gage Height, } \\
\text { feet }\end{array}$ & $\begin{array}{c}\text { Discharge, } \\
\text { cubic feet per } \\
\text { second }\end{array}$ \\
\hline 1.80 & 0.15 & 2.60 & 15.0 \\
1.86 & 0.3 & 2.80 & 23.8 \\
1.90 & 0.5 & 3.00 & 34.5 \\
1.95 & 0.8 & 3.30 & 55.0 \\
2.00 & 1.1 & 3.60 & 80.0 \\
2.06 & 1.6 & 4.00 & 120 \\
2.12 & 2.3 & 4.50 & 182 \\
2.19 & 3.3 & & \\
2.23 & 4.0 & & \\
2.28 & 5.0 & & \\
2.32 & 6.0 & & \\
2.40 & 8.2 & & \\
\hline
\end{tabular}


Prepared by the Pembroke Publishing Service Center

For more information concerning the research in this report, contact: Robert M. Lent, Director

U.S. Geological Survey Maine Water Science Center

196 Whitten Rd.

Augusta, ME 04330

or visit our Web site at:

http://me.water.usgs.gov 


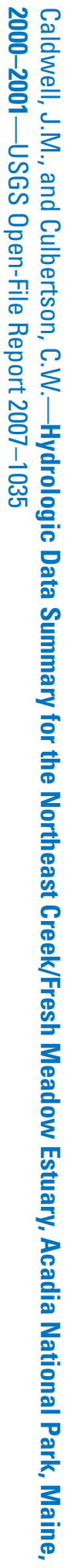

9 Printed on recycled paper 